University of Rhode Island

DigitalCommons@URI

Open Access Dissertations

2015

\title{
APPLYING DIVERSITY TO MITIGATE INTERFERENCE IN UNDERWATER ACOUSTIC COMMUNICATION NETWORKS
}

James A. McGee

University of Rhode Island, jamesamcgee0496@gmail.com

Follow this and additional works at: https://digitalcommons.uri.edu/oa_diss

\section{Recommended Citation}

McGee, James A., "APPLYING DIVERSITY TO MITIGATE INTERFERENCE IN UNDERWATER ACOUSTIC COMMUNICATION NETWORKS" (2015). Open Access Dissertations. Paper 305.

https://digitalcommons.uri.edu/oa_diss/305

This Dissertation is brought to you for free and open access by DigitalCommons@URI. It has been accepted for inclusion in Open Access Dissertations by an authorized administrator of DigitalCommons@URI. For more information, please contact digitalcommons-group@uri.edu. 
APPLYING DIVERSITY TO MITIGATE INTERFERENCE IN UNDERWATER ACOUSTIC COMMUNICATION NETWORKS

BY

JAMES A. MCGEE

A DISSERTATION SUBMITTED IN PARTIAL FULFILLMENT OF THE REQUIREMENTS FOR THE DEGREE OF

DOCTOR OF PHILOSOPHY

IN

ELECTRICAL ENGINEERING

UNIVERSITY OF RHODE ISLAND

2015 


\section{DOCTOR OF PHILOSOPHY DISSERTATION \\ $\mathrm{OF}$}

JAMES A. MCGEE

APPROVED:

Dissertation Committee:

$\begin{array}{ll}\text { Major Professor } & \text { Peter F. Swaszek } \\ & \text { Steven M. Kay } \\ & \text { Manbir Sodhi } \\ & \text { Nasser H. Zawia } \\ & \text { DEAN OF THE GRADUATE SCHOOL }\end{array}$

UNIVERSITY OF RHODE ISLAND 


\begin{abstract}
Many acoustic channels suffer from interference which is neither narrowband nor impulsive. This relatively long duration partial band interference can be particularly detrimental to system performance. In undersea networks, many dropped messages are lost due to partial band interference which corrupts different portions of the received signal depending on the relative position of the interferers, information source and receivers due to the slow speed of propagation. A survey of recent work in interference mitigation and orthogonal frequency division multiplexing (OFDM) provides motivation to develop a spatial diversity receiver for use in underwater networks. The spatial diversity receiver for underwater communications identifies portions of the signal suffering from interference on different receivers, removes these portions of the signal and then optimally combines the remaining clean portions of the signal. The gain of the spatial diversity combining strategy is investigated as a function of signal to interference ratio (SIR), signal to noise ratio (SNR), interference bandwidth and time duration. Analytic results of performance for receivers on additive white Gaussian noise channels suffering time orthogonal interference demonstrate the effectiveness of the spatial diversity combining strategy as compared to conventional maximum ratio combining. Simulation results on time invariant channels confirm the effectiveness of the algorithm under more complex channel conditions. Comparison of the spatial diversity receiver performance to a recently developed single receiver parameterized interference cancellation algorithm is made using experimental data collected on the Atlantic Undersea Test and Evaluation Center (AUTEC) network which consists of multiple distributed cabled hydrophones. Both techniques are effective strategies for combating interference but approach the problem of interference in fundamentally different ways: the spatial diversity receiver blanks the interference while the cancellation algorithm
\end{abstract}


coherently removes it from the desired signal. Spatial diversity reconstruction is effective and realizes the most gain at low SIR and moderate SNR while parameterized interference cancellation is most effective at moderate SIRs and SNRs. The two approaches are complimentary and an effective multi-channel receiver strategy would be to adaptively utilize both techniques. 


\section{ACKNOWLEDGMENTS}

The contribution that this work makes to the existing ocean of knowledge about underwater communications is only possible because of the support, expertise, guidance, love, and just plain presence of many people in my life. I am grateful for all that I have been blessed to receive. Calling to mind these people, and the journey they have accompanied me on, is at once inspiring and humbling.

My adviser, Professor Swaszek, is bright, witty, thoughtful and caring. Somehow he always managed to coax me back from the acute corner of the 13-sided polygon, where I found myself struggling, to the middle of a sandbox where I could play - and somehow always done with humor and patience.

I am grateful to the members of my committee: Professor Kay, Professor Sodhi, Professor Vaccaro, Professor Greenfield and Professor Meyer. Dr. Kay introduced me to probability and random processes, a subject I find fascinating. I enjoyed the back and forth in the optimization course I took with Dr. Sodhi.

The University of Rhode Island has been a welcoming place to learn and grow. Every class has opened my mind and introduced me to people who have enriched my life. I am especially thankful I had the chance to study probability with Professor Pakula. Thank you to Professor Kook for the hours spent teaching me how to count in combinatorics. Ashwin Sarma brings a rare depth of creativity and thoughtfulness to the classroom. My first steps on this road were taken with Dr. Stepanishen. I am grateful our paths still periodically intersect.

The Navy, and the Naval Undersea Warfare Center in particular, fully supported my efforts to study. I am grateful to my friends, colleagues and mentors there. I am grateful to Josko Catipovic for his guidance and for ensuring I had the time and space to pursue my research. Thank you to my branch head, Holly Johnson, for all of the support you have provided. Thank you to Joe, Tod, Phillip 
and Chris for the opportunity to do interesting technical work and the mentoring you provided along the way. Joe, I am especially grateful for our friendship.

Sound propagating in the ocean bends, reflects, scatters; its path winds, is rarely straight. Life is similarly non-linear, full of randomness and the unexpected. There are people who make life seem simpler and fuller and more beautiful: I am grateful to my sister and brother, Sarah, Ari, Leslie and Gladys. I am blessed with many truly remarkable friendships. There are those friends who I don't see for a while, but actually are right here because they show up in an instant: Adam, John, Jeff, Rob, Ed and Ed; and Josh, Terry, Jeremy, Barry, Dale, Dave and Dave. Meghan, I am thankful you took combinatorics. Sometimes tennis isn't really about tennis: thanks George and Frank et al. Karina, I am grateful for our runs.

Steve, you show up no matter what: there is nothing more a person can give than that.

I am grateful for my family. To my parents, for their love and support in every endeavor I have undertaken. To my brother and sister, I know you always have my back. This doesn't happen without all of you.

All of these people nourish me, seeing something in me that I sometimes struggle to see myself.

Finally, I am grateful to Kim for the love and support and care she provides to Annelise, Hugh and Elsa. Our children love and need their Mom. 


\section{DEDICATION}

For Annelise, Hugh and Elsa,

May your lives be filled with meaningful communications.

Love,

Dad 
In order to arrive there,

To arrive where you are, to get from where you are not,

You must go by a way wherein there is no ecstasy.

In order to arrive at what you do not know

You must go by a way which is the way of ignorance.

In order to possess what you do not possess

You must go by the way of dispossession.

In order to arrive at what you are not

You must go through the way in which you are not.

And what you do not know is the only thing you know

And what you own is what you do not own

And where you are is where you are not. ${ }^{1}$

${ }^{1}$ excerpt from "Burnt Norton" by T. S. Eliot 

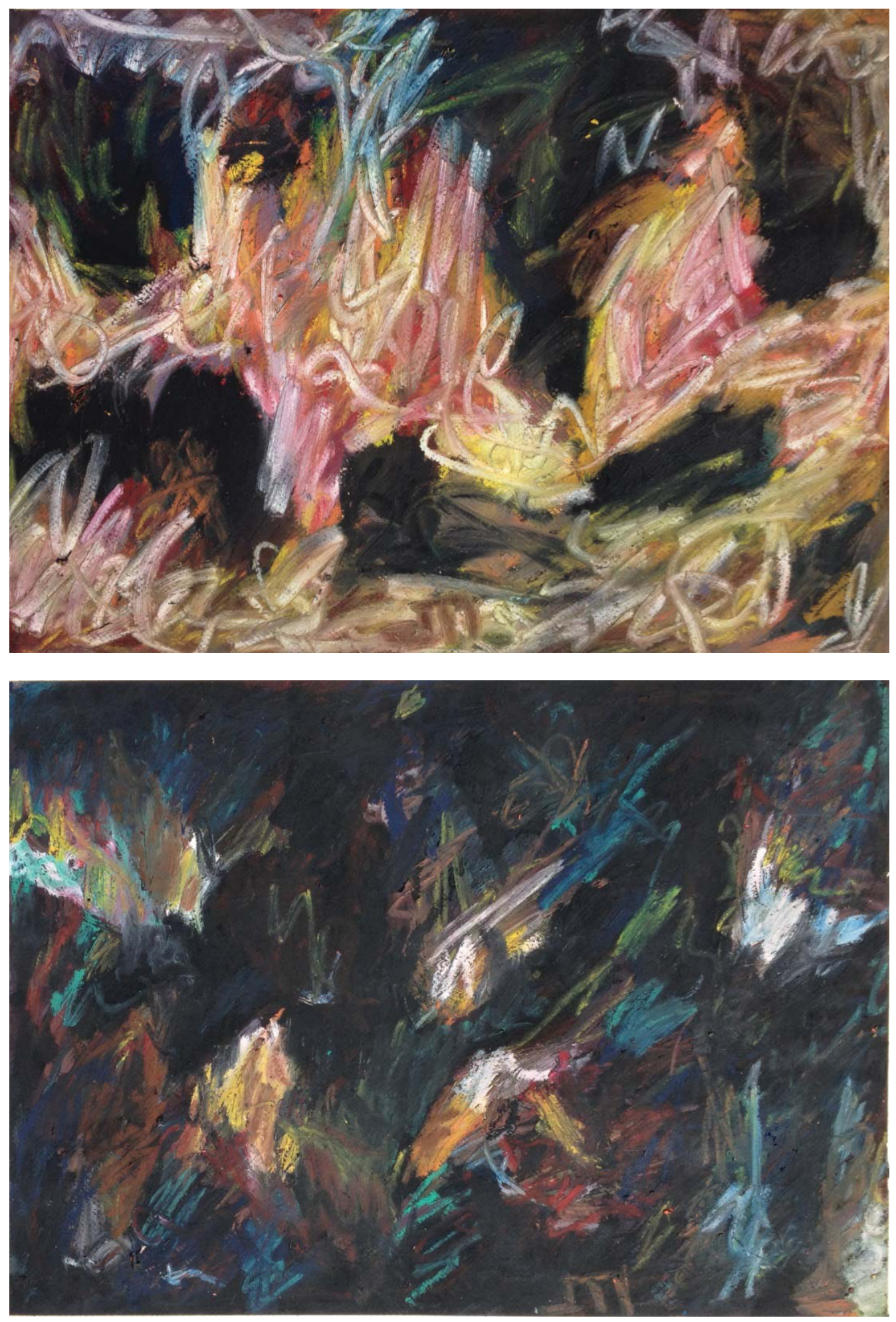


\section{TABLE OF CONTENTS}

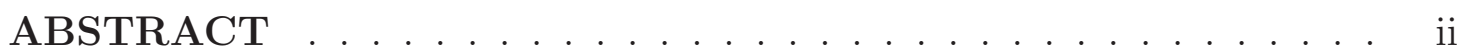

ACKNOWLEDGMENTS .................. iv

DEDICATION ............................ vi

TABLE OF CONTENTS ................. . ix

List of Tables . . . . . . . . . . . . . . . . . xiii

List of Figures $\ldots \ldots \ldots \ldots \ldots \ldots \ldots \ldots \ldots \ldots \ldots \ldots$

Chapter

1 Acoustic Communication Channels and Interference . . . . 1

1.1 Background . . . . . . . . . . . . . . . . . 1

1.2 Challenges of the Underwater Channel . . . . . . . . . . . 3

1.3 The Physics of Underwater Sound Propagation and Underwater

Communication Channel Models . . . . . . . . . . 7

1.3.1 The Wave Equation . . . . . . . . . . . . . . 7

1.3.2 Model of the Underwater Communication Channel . . . 10

1.4 Introduction to Orthogonal Frequency Division Multiplexing

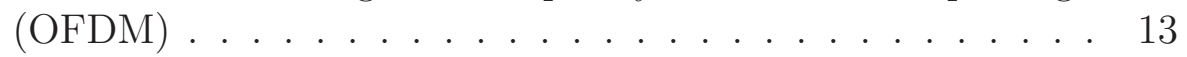

1.4.1 Transmitted OFDM Signal . . . . . . . . . . . . . 18

1.4.2 Channel Model and Received Signal . . . . . . . . . . 19

1.5 Survey of Recent Work in OFDM and Interference Mitigation . 23

1.6 Motivation for the Research . . . . . . . . . . . . . . . . . 29

1.7 Summary . . . . . . . . . . . . . . . . . . 33 


\section{Page}

2 Spatial Diversity Receiver ................ 34

2.1 Introduction . . . . . . . . . . . . . . . . 34

2.2 Spatial Diversity at AUTEC . . . . . . . . . . . . . . 34

2.3 A Spatial Diversity Receiver to Mitigate Interference . . . . . . 35

2.4 Spatial Diversity Reconstruction for OFDM . . . . . . . . . . 38

2.4.1 Frequency Domain Interference Detection . . . . . . . . . 41

2.4.2 Channel Equalization . . . . . . . . . . . . . 42

2.4.3 Time Domain Waveform Reconstruction . . . . . . . . 43

2.4.4 Re-equalization, Data Detection and Decoding . . . . . . 47

2.5 Sources of Error . . . . . . . . . . . . . . . . . . . . 48

2.6 Mitigation of Partial Band Partial Block Interference through Coherent Cancellation . . . . . . . . . . . . . 52

2.6.1 Interference Parameterization . . . . . . . . . 56

2.6.2 Interference Detection and Estimation . . . . . . . 57

2.6.3 Channel Estimation, Equalization and LDPC Decoding . 59

2.6.4 Noise Variance Estimation . . . . . . . . . . . . 60

2.7 Comparison of Spatial Diversity Reconstruction and Parameterized Interference Cancellation . . . . . . . . . . . . 61

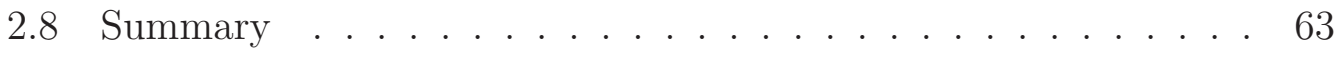

3 Simulation and Experimental Results . . . . . . . . . . . 65

3.1 Introduction . . . . . . . . . . . . . . . . . . 65

3.2 Performance on Additive White Gaussian Noise Channel with Interference ................. . . . 65

3.2.1 Additive White Gaussian Noise and Time Orthogonal Interference Channel Model . . . . . . . . . . . . 67 


\section{Page}

3.2.2 Spatial Diversity Reconstruction Performance . . . . . . 69

3.2.3 Maximum Ratio Combining Performance . . . . . . . . . 72

3.2.4 Example: Two Receivers on Additive White Gaussian Noise Channel with Interference . . . . . . . . . . 73

3.2.5 Example: Three Receivers on Additive White Gaussian Noise Channel with Interference . . . . . . . . . 75

3.3 Simulation Results on a Time Invariant Channel . . . . . . . . . 81

3.4 Experimental Results . . . . . . . . . . . . . . . . . . 85

3.4.1 Example of SDR on Experimental Data . . . . . . . . 87

3.4.2 Statistical Performance of Algorithms Based on PseudoExperimental Data . . . . . . . . . . . . 88

3.5 Summary . . . . . . . . . . . . . . . . . . . . . . . 93

4 Interference Experiment at AUTEC December $2014 \ldots \ldots$

4.1 Introduction $\ldots \ldots \ldots \ldots \ldots \ldots \ldots$

4.2 Experiment Objectives . . . . . . . . . . . . . . . . 95

4.3 Experiment Procedure . . . . . . . . . . . . . . . . . . . . . 98

4.3.1 OFDM Communication Signal and LFM Interference . . 102

4.4 Data Collected . . . . . . . . . . . . . . . . . . . . 109

4.5 Channel Model . . . . . . . . . . . . . . . . . . . . . . . . 111

4.5.1 Physical Basis for Channel Model . . . . . . . . . . . . 118

4.6 Summary . . . . . . . . . . . . . . . . . . . . . . . 121

5 Conclusions and Future Work . . . . . . . . . . . . . . . 123

5.1 Conclusions . . . . . . . . . . . . . . . . . . . . . . 123

5.2 Future Work . . . . . . . . . . . . . . . . . . . 126 
LIST OF REFERENCES . . . . . . . . . . . . . . . . . . 130 APPENDIX

AUTEC Experiment in May 2014 (AUTEC-0514) . . . . . . . . . 138

A.1 Description of the AUTEC May 2014 Experiment . . . . . . . . 138

A.1.1 Generation of Pseudo-Experimental Data . . . . . . . . . 141

A.2 MATLAB $^{\circledR}$ Code . . . . . . . . . . . . . . . . . . . . 147

BIBLIOGRAPHY . . . . . . . . . . . . . . . . . . . 157 


\section{List of Tables}

$\begin{array}{lll}\text { Table } & \text { Page }\end{array}$

1 Comparison of the interference mitigation algorithms . . . . 63

2 Parameters for simulation of AWGN channel with interference . 78

3 Parameters for simulation of linear time invariant channel. . . 85

4 AUTEC-0514 OFDM and LFM transmit parameters. . . . . 87

$5 \quad$ Distance from receivers to transmitters 69 and $75 \ldots \ldots 100$

6 OFDM signal with LFM interference wavefiles. . . . . . . 103

$7 \quad$ Type of interference expected on each block for various receivers. 104

8 AUTEC December 2014 OFDM and LFM transmit parameters. 107

A.1 OFDM signal with LFM interference wavefiles. . . . . . . . 140

A.2 AUTEC-0514 OFDM and LFM transmit parameters. . . . . . 141 


\section{List of Figures}

Figure

Page

1 The AUTEC acoustic network. . . . . . . . . . . . . 4

$2 \quad$ Clean packet reception (a) and packet corrupted by acoustic interference $(\mathrm{b}) \ldots \ldots \ldots \ldots$

3 An OFDM waveform consisting of four subcarriers. . . . . . . . 14

4 An OFDM data block. . . . . . . . . . . . . 15

5 An OFDM data packet. ................. 15

6 CP-OFDM and ZP-OFDM transmitter processing. . . . . . . 16

$7 \quad$ CP-OFDM and ZP-OFDM receiver processing. . . . . . . . . 17

8 ZP-OFDM receiver for underwater acoustic communications. . . 19

9 Two received OFDM data blocks suffering from interference which corrupts the receptions at different times. . . . . . . . . 31

10 Received signal constellations resulting from processing the receptions in Fig. 9 using different combining techniques. . . . . . 32

11 Receptions as a function of geometry from the transmitter. . . . 36

$12 \quad$ Spatial Diversity Reconstruction (SDR) processing. . . . . . . . 39

13 Illustration of the combining strategy for the case of three receivers. . . . . . . . . . . . . . . . 40

14 The SDR time domain interference detection windows. . . . . . 44

15 Weighted combining for 3 receivers suffering non-orthogonal time domain interference. . . . . . . . . . . . 46

16 SDR processing a single carrier with $50 \%$ amplitude mismatch. $\quad 50$

17 SDR processing a single carrier with $20^{\circ}$ phase mismatch. . . . 51

18 SDR processing an OFDM waveform with $20^{\circ}$ phase mismatch. 53 
19 Signal constellation resulting from SDR processing with phase mismatch. ................ . . . 54

20 Signal constellation resulting from SDR processing with amplitude and phase mismatch.. . . . . . . . . . . . . . . 54

21 SDR Processing with various mismatches. . . . . . . . 55

22 SDR combining strategy for three receivers with time orthogonal interference................. . . 70

23 Performance on AWGN channel for two receivers suffering equivalent noise and interference levels. . . . . . . . . . . . . . 76

24 Comparison of MRC and clairvoyant blanking performance for two receivers suffering equivalent noise and interference levels of various bandwidths and time durations. . . . . . . . . . . . 77

25 Performance on AWGN channel as a function of SIR. . . . . . . 79

26 Performance on AWGN channel as a function of SNR. . . . . . 80

27 Simulation geometry. $D\left(s, r_{1}\right)=D\left(s, r_{2}\right)$ and $D\left(i, r_{1}\right)<D\left(i, r_{2}\right) . \quad 82$

28 Simulated channels. . . . . . . . . . . . . . . 83

29 Two receiver performance on time invariant channel . . . . . . . 84

30 Performance comparison between time invariant channel and AWGN channel . . . . . . . . . . . . . . . . . . 86

31 The OFDM signals received on channels 68 (panel a) and 76 (panel b) during AUTEC-0514. . . . . . . . . . . . . 89

32 Uncoded bit error rate performance as a function of SIR for different mitigation strategies. . . . . . . . . . . . . . 90

33 Coded bit error rate performance as a function of SIR for different mitigation strategies. . . . . . . . . . . . . . 90

34 Spectrograms and time series of receptions from five different receivers recorded during the May experiment (AUTEC-0514).

35 Relative position of transmitters and receivers in AUTEC experiments. . . . . . . . . . . . . . . 101 
36 Uncoded bit error rate on simulated channel with no interference mitigation. .................. 108

37 Presence of interference on various receivers. . . . . . . . . . 110

38 Received data packets from various receivers during AUTEC1214 . . . . . . . . . . . . . . . . . . 112

39 Receptions of data packets 1-4 on various receivers from AUTEC-1214. ...................... 113

40 Reception of a data block on receiver 76 from AUTEC-0514 (panel a) and AUTEC-1214 (panel b). . . . . . . . . . . . . 114

41 AUTEC-0514, AUTEC-1214 and other selected sound velocity profiles from AUTEC. . . . . . . . . . . . . 116

42 Channel models from nodes 75 and 69 to various receivers. . . . 117

43 Histograms of the difference in arrival time of direct path and surface bounce receptions of the channel probe. . . . . . . . 118

44 Peak matched filter output for direct path and surface bounce receptions of the channel probe. . . . . . . . . . . 119

45 Direct path and surface bounce rays propagating from transmitter to receiver. . . . . . . . . . . . . 120

$46 \quad$ Spatial diversity receiver. . . . . . . . . . . . 127

A.1 Relative position of transmitters and receivers in the AUTEC0514 experiment. . . . . . . . . . . . . . . . . . . . 139

A.2 Representative time series of the transmitted LFM interference and OFDM signal in the AUTEC-0514 experiment. . . . . . . . 140

A.3 Processing to extract sea noise from received data packets. . . 143

A.4 Processing to extract interference and sea noise from received data packets. . . . . . . . . . . . . . . . 145

A.5 Interference extracted from channel probe and faired into background noise. . . . . . . . . . . . . . . 146 
A.6 Two examples of synthesized experimental data with time orthogonal interference. . . . . . . . . . . . . . 146 


\section{CHAPTER 1}

\section{Acoustic Communication Channels and Interference}

\section{$1.1 \quad$ Background}

While humans have recognized for millennia that sound can be heard underwater, the ability to communicate underwater over long distances is a recent achievement. Overviews of the challenges and technical accomplishments in underwater communications may be found in [1-12]. One of the first Underwater Acoustic Communication systems, developed by the Submarine Signal Company in the early 1900s, attempted to warn ships of navigation hazards by detecting signals transmitted from underwater bells placed in the vicinity of lightships or lighthouses on shipboard receivers. Unfortunately the receivers suffered from such high ambient noise that they could not reliably detect the transmitted signal. Requested by the Submarine Signal Company to improve its receiver, Reginald A. Fessenden developed an electro-mechanical device, eventually known as the Fessenden Oscillator, capable of both producing and detecting sound. In 1913, he used the device to transmit Morse code messages over several miles between two tugboats in Boston Harbor [13].

Today, a reliable acoustic data link is of great importance for the development of ocean-observation systems and sensor networks. Applications abound, ranging from the oil industry to aquaculture, including instrument monitoring, pollution control, climate recording, search and survey missions, the study of marine life, to military applications [12]. The current vision is for the development of an undersea sensor network, specifically a collection of bottom-mounted instruments and tetherless moving platforms in which different kinds of oceanographic data will be exchanged among the members in real time [14]. Major motivations for the use of wireless communications in the undersea network include 1) reduction 
in deployment costs and 2) the operational flexibility provided by the ability to monitor and react to sensor information in real or near-real time [12].

Research into the physical layer of undersea networks is extremely active with focus areas including: the improvement of single carrier modulation/detection with more powerful coding techniques and turbo equalization; the exploration of multi-carrier modulation as an alternative to single carrier; the extension of single-input/single-output (SISO) systems to multi-input/multi-output (MIMO) configurations that provide spatial multiplexing and the ability to send parallel data streams from multiple transmitters [12].

Yet, the deployment of undersea networks is in its infancy. A few short duration experimental networks have been deployed. For example, the Seaweb deployment in 2000 involved 17 nodes spread over $16 \mathrm{~km}^{2}$ for multiple days [7]. The Massachusetts Institute of Technology and the Commonwealth Scientific and Industrial Research Organisation (CISRO) of Australia deployed a network consisting of both fixed and autonomous nodes in very near-shore areas of Australia [15].

To date the only long term undersea network is operated by the U. S. Navy in the Tongue of the Ocean [16]. It consists of 96 hydrophones placed over a $100 \mathrm{~km}^{2}$ area and is shown in Fig. 1. As currently configured, approximately 97 percent of transmitted messages are successfully decoded; of the remaining three percent, many are corrupted by acoustic interference arising from active acoustic emissions. For example, Fig. 2 shows the impact of interference on a received data packet. In panel (a), the data packet was received without interference and successfully decoded in contrast to panel (b) where interfering signals are clearly evident and the message was lost. Years of extensive observations of activities in the vicinity of the network demonstrate that the widely separated hydrophones suffer from partialband interference emanating from multiple spatially separated sources. The nature 
of this interference is different from the impulsive or narrowband interference typically encountered in other applications. Furthermore, unlike RF communications and acoustic array processing applications where interference is highly correlated in time among the various receivers, in the acoustic network interference affects different portions of the received signals due to the wide separation of the receivers and the low speed of propagation. The degradation in the received signal is highly variable, depending on the relative position of the interferers, information source and receivers as well as the channel conditions. While successful steps to mitigate interference have recently been reported [17], utilizing the spatial diversity implicit in the undersea network to mitigate interference has not yet been attempted. The motivation of this work is to examine the potential benefits that leveraging spatial diversity in underwater acoustic networks might provide.

\subsection{Challenges of the Underwater Channel}

While the spatial diversity implicit in the network offers the opportunity to mitigate interference, the diversity receiver must address the challenges the underwater acoustic channel presents, namely: 1) severely limited operational bandwidth, 2) significant multipath spread, 3) non-stationary statistics, and 4) the need for explicit time synchronization in mobile applications (see e.g. $[3,6,19,20]$ ). As is the case at AUTEC, many acoustic channels suffer from interference which is neither narrowband nor impulsive [17]. This relatively long duration partial band interference can be particularly detrimental to communication systems that do not account for it. The challenges the underwater channel presents are manifold, but the diversity inherent in the channel offers opportunities to ameliorate them.

Diversity techniques are well known to improve the reliability of communications over fading multipath channels and rely on the notion that errors occur in reception when the channel attenuation is large; that is, when the channel is in a 


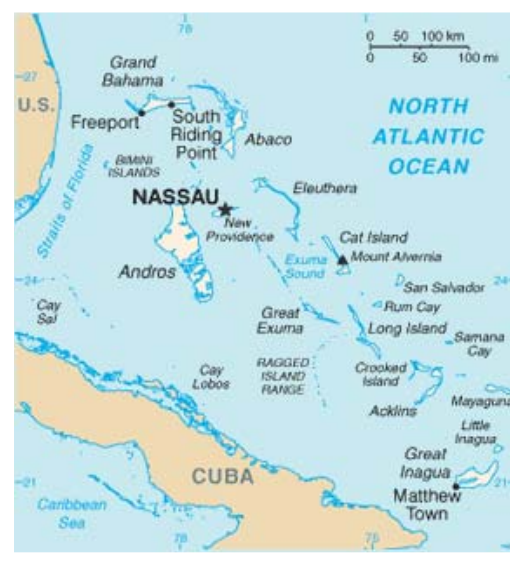

(a)

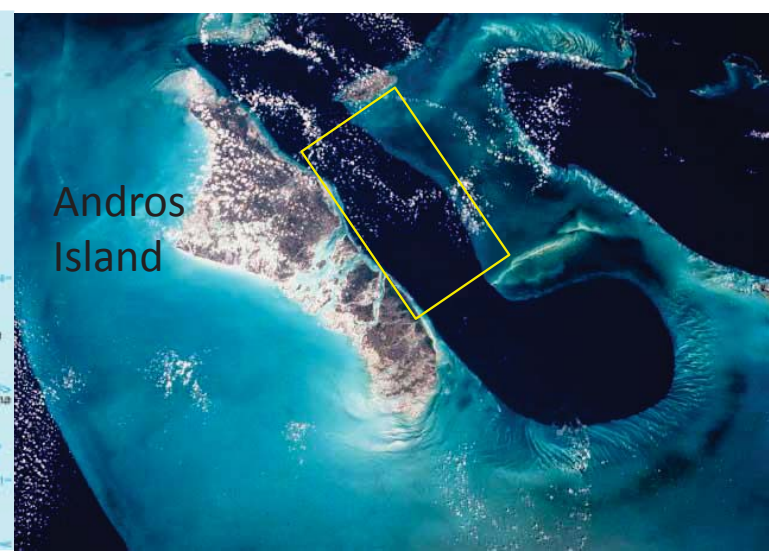

(b)

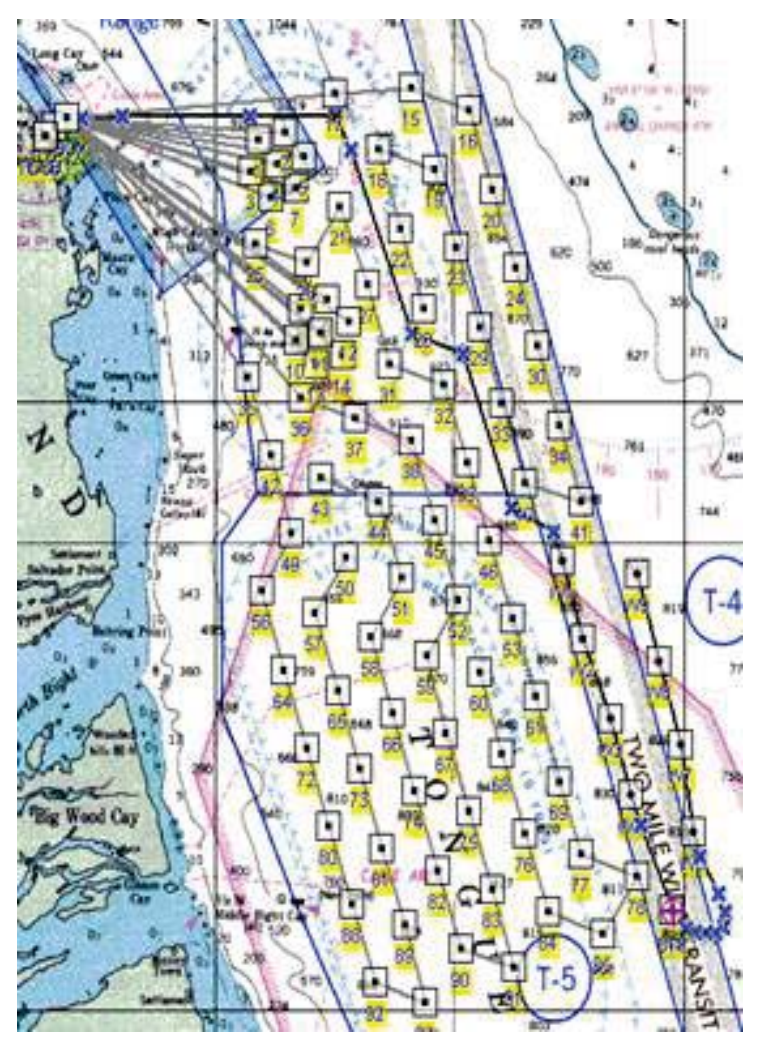

(c)

Figure 1: The AUTEC acoustic network.

AUTEC is located off the coast of Andros Island in the Bahamas. The squares with circles (in panel (c)) indicate the hydrophone locations. Panel (a) is from [18]. 


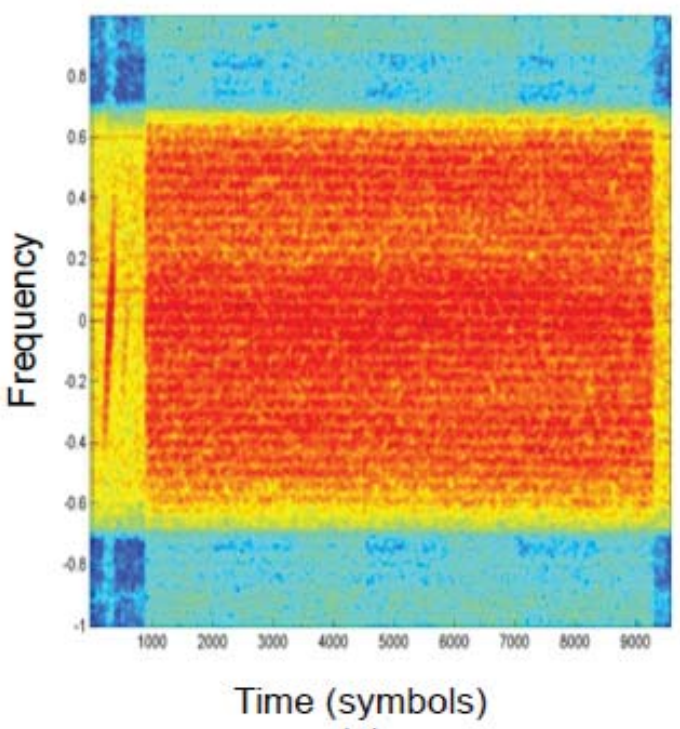

(a)

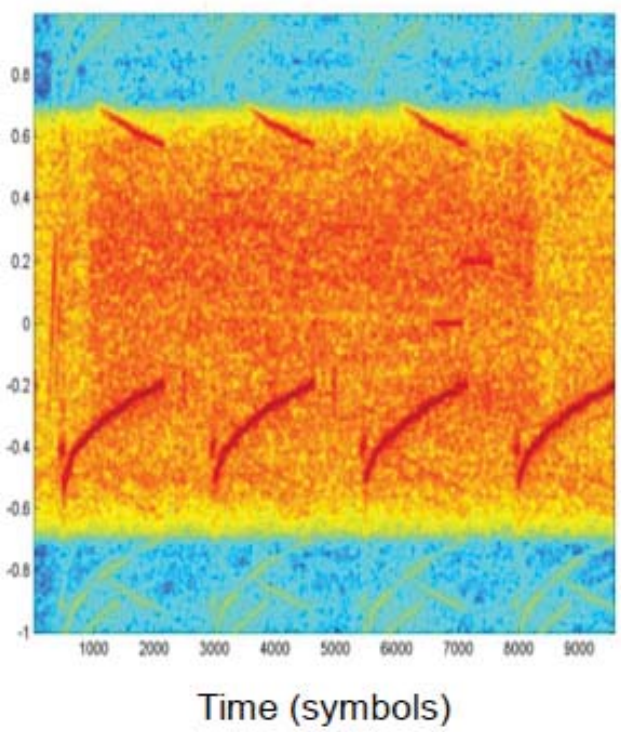

(b)

Figure 2: Clean packet reception (a) and packet corrupted by acoustic interference (b).

deep fade [21]. Diversity techniques may be classified as either explicit, where the message is intentionally transmitted through different uncorrelated parts of the signal space, or implicit, where the message occupies the entire signal spectrum and the receiver tries to identify it by compensating for the channel distortion [22]. The central idea behind both approaches is to supply the receiver with multiple replicas of the same information transmitted over independently fading portions of the channel thereby reducing the probability that all signal components will fade simultaneously.

Diversity techniques can be employed on selective channels, where selectivity refers to the differential enhancement or attenuation exhibited in the received signal resulting from transmission through the channel with respect to a particular parameter. For example, a frequency selective channel attenuates different frequency bands of the transmitted signal differently, and its frequency response varies over its bandwidth. The underwater acoustic channel exhibits time, frequency and spa- 
tial selectivity as a consequence of the physics of sound propagation in the ocean. Frequency selectivity arises from the absorption losses which increase with signal frequency. The coherence bandwidth of the channel, the range of frequencies over which the channel gain is almost constant, is on the order of $10 \mathrm{~Hz}$ at a transmit frequency of $10 \mathrm{kHz}$. Time and spatial selectivity result from time-varying multipath propagation, and the low speed of sound (1500 mps). Multipath formation is governed by sound reflection and refraction. Reflection occurs at the surface and bottom and off any objects in the medium. Refraction results from the spatial variability of sound speed which depends on the temperature, salinity, and pressure. The multipath delay spread, the time delay between the first and last significant arrival of a transmitted signal, can range from 10 milliseconds in shallow water at short ranges to hundreds of milliseconds in deep ocean channels. The multipath structure is highly environmentally dependent and the impulse response may be sparse exhibiting a few large amplitude taps separated by many taps of small magnitude.

Changes in the propagation medium and transmitter/receiver motion induce time variability in the channel. Changes in the propagation medium occur over different time scales ranging from seasonal temperature changes to much faster fluctuations that affect the signal such as surface wave action. Wave motion causes scattering of the signal and rapid fluctuations in signal path length induces Doppler spreading. The slow speed of sound relative to the speed of mobile transmitters results in significant variability to the channel through the Doppler effect which causes frequency shifting as well as frequency spreading. The resulting channel exhibits significant time variability. Coherence time, a measure of the time scale over which the channel changes significantly, can range from seconds in extremely stable situations to 100 milliseconds in rapidly fluctuating channels. 


\subsection{The Physics of Underwater Sound Propagation and Underwater Communication Channel Models}

This section introduces the wave equation which governs the propagation of sound in water and describes typical acoustic communication channel models. No acoustic communication systems attempt to solve the wave equation directly. However, all acoustic communication systems must operate in an environment governed by it.

\subsubsection{The Wave Equation}

The ocean forms an acoustic waveguide bounded above by the sea surface and below by the sea floor. The sound speed of the medium, discontinuities within it and boundary conditions interact in a rich and complex manner to affect the transmission of sound in the waveguide. Despite the richness and complexity of these interactions, mathematical models exist which can predict and explain the observed sound propagation behavior to a certain extent. The starting point for these models is the solution of the wave equation with various simplifying assumptions. While the focus of this work is the development of an acoustic communications receiver which avoids solving the wave equation directly, understanding it provides insight into the physics of how spatial variability arises in the propagation of sound. The following discussion draws on the work in [23] and [24].

The wave equation in an ideal fluid can be derived from hydrodynamics and

the adiabatic relationship between pressure and density. The equation for the conservation of mass, Euler's equation (Newton's 2nd Law), and the adiabatic equation of state are 


$$
\begin{aligned}
\frac{\partial \rho}{\partial t} & =-\nabla \cdot \rho \mathbf{v} \\
\frac{\partial \mathbf{v}}{\partial t}+\mathbf{v} \cdot \nabla \mathbf{v} & =-\frac{1}{\rho} \nabla p(\rho) \\
p & =p_{0}+\rho^{\prime}\left[\frac{\partial p}{\partial \rho}\right]_{S}+\frac{1}{2}\left(\rho^{\prime}\right)^{2}\left[\frac{\partial^{2} p}{\partial \rho^{2}}\right]_{S}+\cdots
\end{aligned}
$$

These equations relate time and spatial changes in density $\rho$, particle velocity $\mathbf{v}$ and pressure $p$. The operator $[\cdot]_{S}$ is the thermodynamic quantity calculated at constant entropy. The speed of sound in an ideal fluid is related to the density and pressure or bulk modulus of elasticity through

$$
c^{2}=\left[\frac{\partial p}{\partial \rho}\right]_{S}=\frac{B}{\rho} .
$$

The ambient quantities of the quiescent (time independent) medium are identified by the subscript 0 . Small perturbations for the pressure and density are denoted by primes, that is $p=p_{0}+p^{\prime}$ and $\rho=\rho_{0}+\rho^{\prime}$. The particle velocity $\mathbf{v}$ due to density and pressure fluctuations is much smaller than the speed of sound.

Retaining only the first order terms in the hydrodynamic equations leads to the linear wave equation. To the lowest order Eqs. (1)-(4) become

$$
\begin{aligned}
\frac{\partial \rho^{\prime}}{\partial t} & =-\nabla \cdot \rho_{0} \mathbf{v} \\
\frac{\partial \mathbf{v}}{\partial t} & =-\frac{1}{\rho_{0}} \nabla p^{\prime}(\rho) \\
p^{\prime} & =\rho^{\prime} c^{2}
\end{aligned}
$$

The material properties $\rho_{0}$ and $c^{2}$ may be assumed independent of time provided the time scale of acoustic propagation is much shorter than the time scale of 
oceanographic changes. Taking the partial derivative of Eq. (5) with respect to time and the divergence of Eq. (6) yields:

$$
\begin{aligned}
\frac{\partial^{2} \rho^{\prime}}{\partial t^{2}} & =-\frac{\partial\left(\rho_{0} \nabla \cdot \mathbf{v}\right)}{\partial t}=-\rho_{0} \nabla \cdot \frac{\partial \mathbf{v}}{\partial t} \\
\nabla \frac{\partial \mathbf{v}}{\partial t} & =-\frac{1}{\rho_{0}} \nabla^{2} p^{\prime}(\rho) .
\end{aligned}
$$

and combining Eqs. (8) and (9) produces:

$$
\frac{\partial^{2} \rho^{\prime}}{\partial t^{2}}-\rho_{0} \nabla \cdot \frac{1}{\rho_{0}} \nabla p^{\prime}(\rho)=0 .
$$

Applying the linearized adiabatic equation of state, Eq. (7), removing the primes and letting $\rho_{0}=\rho$ results in the linearized wave equation for pressure:

$$
\begin{aligned}
\frac{\partial^{2}\left(p / c^{2}\right)}{\partial t^{2}}-\rho \nabla \cdot \frac{1}{\rho} \nabla p(\rho) & =0 \\
\frac{1}{c^{2}} \frac{\partial^{2} p}{\partial t^{2}}-\rho \nabla \cdot \frac{1}{\rho} \nabla p(\rho) & =0 .
\end{aligned}
$$

Note that the derivation assumes that density is constant with respect to the time scale of interest but may vary spatially. If the density exhibits no spatial variability, then the standard form of the wave applies,

$$
\frac{1}{c^{2}} \frac{\partial^{2} p}{\partial t^{2}}-\nabla^{2} p(\rho)=0
$$

While the wave equation describes the physics of sound propagation, solving it is not an easy task. A number of computer algorithms such as Kraken [25-30] or Bellhop [31] have been developed to do this. However, the results rely on the estimates of spatially and time varying parameters of the ocean which are difficult to obtain accurately. Attempts to incorporate detailed knowledge of the environment into signal processing algorithms have met with only limited success. Processing algorithms that avoid solving the wave equation directly but account 
for the general characteristics of underwater sound propagation have been more fruitful. The channel model discussed in the next section enables the development of such algorithms.

\subsubsection{Model of the Underwater Communication Channel}

The underwater acoustic channel can be modeled as a linear, time-varying system which is described by the channel impulse response $h(t ; \tau)$, where $t$ is the "short-time" variable corresponding to time variations of the impulse response due to moving platforms and physical boundaries and $\tau$ is the "age" (or elapsed-time) variable corresponding to the multipath delay for a fixed value of $t$. In the absence of noise, the received bandpass signal $\tilde{y}(t)$ is determined by the convolution of the channel impulse response with the transmitted bandpass signal $\tilde{s}(t)$ :

$$
\begin{aligned}
\tilde{y}(t) & =h(t ; \tau) \star \tilde{s}(t) \\
& =\int h(t ; \tau) \tilde{s}(t-\tau) d \tau .
\end{aligned}
$$

Alternatively, Eq. (14) may be interpreted as a system with impulse response $h(t ; \tau)$ at time $t$ to an impulse that is applied at time $t-\tau$.

A reasonable model for the underwater acoustic channel (and for other physical channels such as the ionosphere at frequencies below $30 \mathrm{MHz}$ and mobile cellular radio) is a special case of Eq. (14) where the impulse response has the form

$$
h(t ; \tau)=\sum_{p=1}^{N_{p a}} \mathcal{A}_{p}(t) \delta\left(\tau-\bar{\tau}_{p}(t)\right) .
$$

Here, the channel consists of $N_{p a}$ discrete paths with path specific time varying amplitudes $\mathcal{A}_{p}(t)$ and delays $\bar{\tau}_{p}(t)$. Further simplifying assumptions can be made depending on the relative time duration of the signal and the coherence time of the channel. When the signal is short compared to the coherence time, the following assumptions may be adopted: 
- AS1): The path amplitudes are constant within a short time period

$$
\mathcal{A}_{p}(t)=A_{p}
$$

- AS2): The delay variation over the time duration of the signal can be approximated by its first-order Taylor series expansion

$$
\bar{\tau}_{p}(t) \approx \bar{\tau}_{p}-a_{p} t, \quad t \epsilon[0, T]
$$

where $\bar{\tau}_{p}$ is the initial delay and $a_{p}$ is the first order derivative of $\bar{\tau}_{p}(t)$ and $T$ is the period of the signal.

The parameter $a_{p}$ is known as the Doppler scaling factor and can be related to the relative speed $v_{p}$ of the transmitter and receiver along the pth path as $a_{p}=\frac{v_{p}}{c}$. Positive values of $a_{p}$ occur for positive relative Doppler shifts resulting in signal contraction while negative values of $a_{p}$ indicate opening relative Doppler causing signal dilation. The Doppler scale factor for a vessel traveling at fifteen knots transmitting acoustic communications to a fixed sensor is approximately $5.1 \times 10^{-3}$ (7.7 mps / $\left.1500 \mathrm{mps}\right)$. The scale factor is much larger in underwater communications compared to mobile radio where the speed of propagation is high and typical receiver speeds ( $29 \mathrm{mps} \approx 65 \mathrm{mph}$ ) produce a Doppler scale factor on the order of $1 \times 10^{-7}$. Based on assumptions AS1 and AS2, the impulse response of the underwater channel with independent Doppler scaling on each path is modeled as

$$
h(t ; \tau)=\sum_{p=1}^{N_{p a}} A_{p} \delta\left(\tau-\left(\bar{\tau}_{p}-a_{p} t\right)\right) .
$$

The received bandpass signal, $\tilde{y}(t)$, is related to the transmitted bandpass signal, $\tilde{s}(t)$, through the convolution of the transmitted signal with the channel 
impulse response with the addition of noise, $\tilde{n}(t)$ and (possibly) interference $\tilde{i}(t)$ :

$$
\begin{aligned}
\tilde{y}(t) & =\int \sum_{p=1}^{N_{p a}} A_{p} \delta\left(\tau-\left(\bar{\tau}_{p}-a_{p} t\right)\right) \tilde{s}(t-\tau) d \tau+\tilde{n}(t)+\tilde{i}(t) \\
& =\sum_{p=1}^{N_{p a}} A_{p} \tilde{s}\left(t-\left(\bar{\tau}_{p}-a_{p} t\right)\right)+\tilde{n}(t)+\tilde{i}(t) \\
& =\sum_{p=1}^{N_{p a}} A_{p} \tilde{s}\left(\left(1+a_{p}\right) t-\bar{\tau}_{p}\right)+\tilde{n}(t)+\tilde{i}(t) .
\end{aligned}
$$

The resulting time-varying channel with path-specific Doppler scales is parameterized by $N_{p a}$ triplets $\left\{A_{p}, a_{p}, \bar{\tau}_{p}\right\}$. The received signal in the frequency domain is found via Fourier transform. Applying the Fourier transform pairs

$$
\begin{array}{lll}
s(t / T) & \leftrightarrow T S(f T) & \text { Scaling of a variable, } T>0 \\
s\left(t-t_{0}\right) \leftrightarrow S(f) e^{-j 2 \pi f t_{0}} & \text { Time translation }
\end{array}
$$

to $\tilde{y}(t)$ yields

$$
\tilde{Y}(f)=\sum_{p=1}^{N_{p a}} \frac{A_{p}}{1+a_{p}} \tilde{S}\left(\frac{f}{1+a_{p}}\right) e^{-j 2 \pi\left(\frac{f}{1+a_{p}}\right) \bar{\tau}_{p}}+\tilde{N}(f)+\tilde{I}(f),
$$

where $\tilde{N}(f)$ and $\tilde{I}(f)$ are the Fourier transforms of $\tilde{n}(t)$ and $\tilde{i}(t)$ respectively.

If the Doppler fluctuations remain relatively constant over a signal period, the channel is parameterized by its common Doppler scale factor, $a$, and the $N_{p a}$ pairs of amplitudes and delays, $\left\{A_{p}, \bar{\tau}_{p}\right\}$. In this case, the received signal is given by

$$
\begin{aligned}
\tilde{y}(t) & =\sum_{p=1}^{N_{p a}} A_{p} \tilde{s}\left(t-\left(\bar{\tau}_{p}-a t\right)\right)+\tilde{n}(t)+\tilde{i}(t) \\
& =\sum_{p=1}^{N_{p a}} A_{p} \tilde{s}\left((1+a) t-\bar{\tau}_{p}\right)+\tilde{n}(t)+\tilde{i}(t) .
\end{aligned}
$$

The frequency domain received signal is found as above,

$$
\tilde{Y}(f)=\sum_{p=1}^{N_{p a}} \frac{A_{p}}{1+a} \tilde{S}\left(\frac{f}{1+a}\right) e^{-j 2 \pi\left(\frac{f}{1+a}\right) \bar{\tau}_{p}}+\tilde{N}(f)+\tilde{I}(f) .
$$




\subsection{Introduction to Orthogonal Frequency Division Multiplexing (OFDM)}

Orthogonal Frequency Division Multiplexing (OFDM) is a multi-carrier communications modulation method commonly used in applications such as digital television, wireless networks, audio broadcasting and 4G mobile applications. Techniques have been developed for it that successfully deal with challenges of the underwater acoustic channel. This section introduces the basic mathematics of OFDM modulation and describes the approach developed in [32] that has been successful in compensating for the Doppler induced signal contraction and dilation.

OFDM divides the channel into multiple parallel narrowband channels through the use of an orthogonal partition [33]. In OFDM, the partitioning is accomplished via the Fourier transform which results in the data being transmitted on multiple mutually orthogonal sinusoids. An example of an OFDM time domain signal comprised of four subcarriers is shown in Fig 3. The subcarriers are shown in color, and the transmitted waveform resulting from adding all of the subcarriers is shown in black. The subcarrier data rate is lower than the total data rate and the subchannel bandwidth is less than the total system bandwidth.

For a given total system bandwidth, the number of carriers determines the bandwidth of the subchannels. Typically the number of carriers is selected to ensure that the bandwidth of the subchannels is less than the coherence bandwidth of the channel so that the subchannels experience relatively flat fading and inter-symbol interference (ISI) is small. Taken together, the subchannels form a OFDM symbol block. Within each block, as shown in Fig. 4, carriers are allocated for data transmission (data carriers), channel estimation (pilot carriers) and noise and car-

rier frequency offset estimation (null carriers). Blocks are stitched together along with a preamble and postamble to form an OFDM data packet. The preamble and postamble are typically Linear Frequency Modulated (LFM) or Hyperbolic 


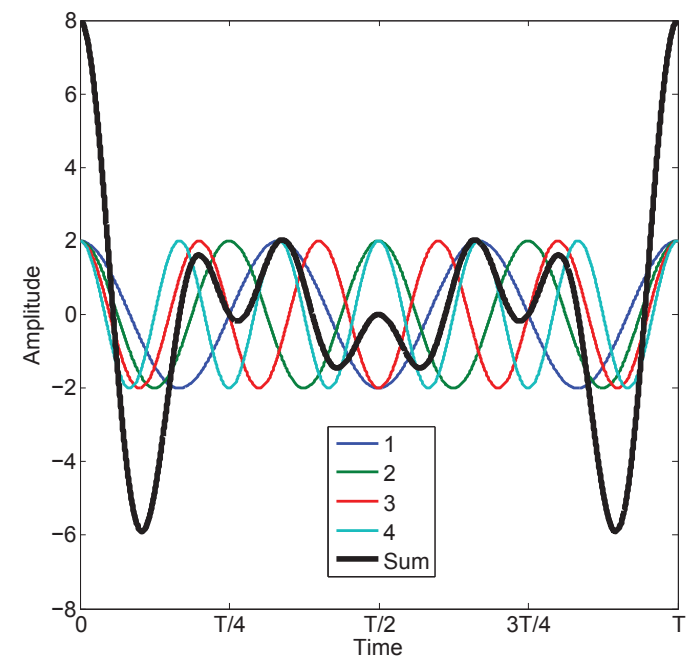

Figure 3: An OFDM waveform consisting of four subcarriers. The subcarriers are shown in color; the OFDM waveform resulting from summing the carriers is shown in black. The transmitted OFDM waveform has duration $T$.

Frequency Modulated (HFM) waveforms and facilitate data detection, synchronization as well as Doppler scale and channel estimation. Some techniques do not require a postamble. For example, [34] structures the preamble so that a bank of parallel correlators, each matched to a different Doppler scaling factor with respect to the waveform dilation or compression, can perform signal detection and Doppler scale estimation.

OFDM comes in two basic flavors: Cyclic Prefix OFDM (CP-OFDM) and Zero Padded OFDM (ZP-OFDM). The two variants differ in the treatment of the interval between data blocks. In CP-OFDM, the guard interval occurs before the data block and is filled with samples from the end of the transmit data sequence as shown in Fig 6. By appending a cyclic prefix to the channel input sequence, the linear convolution of the transmit signal with the channel impulse response becomes a circular convolution (see chapter 12 of [33]). On the receiver side, the samples corresponding to the cyclic prefix are discarded for CP-OFDM and the samples 


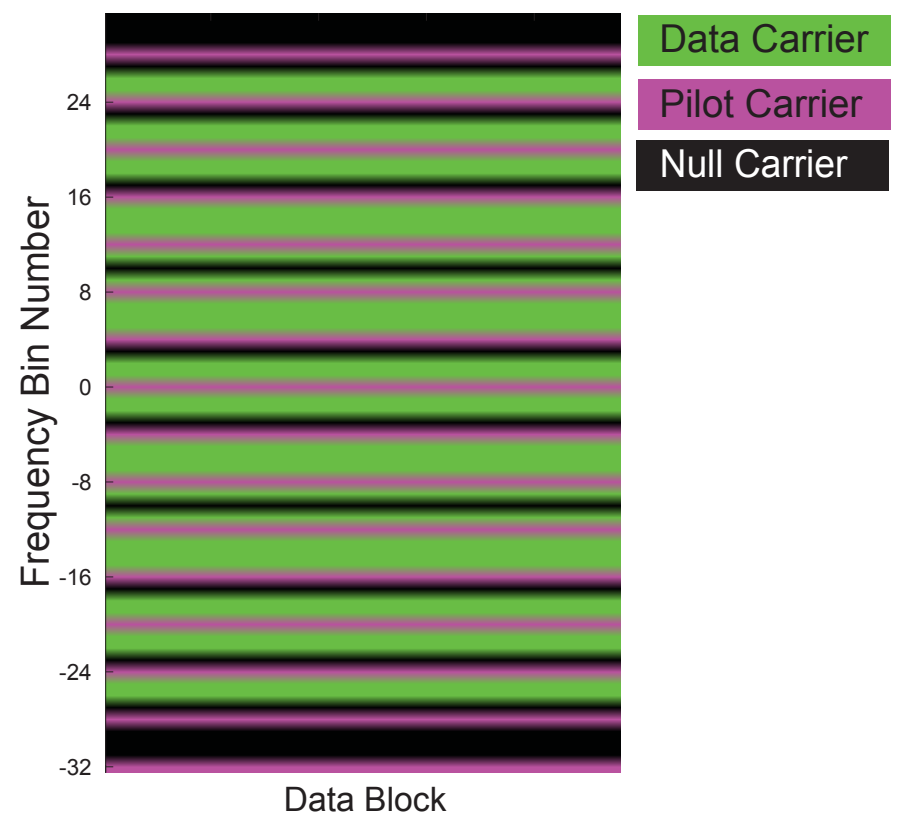

Figure 4: An OFDM data block.

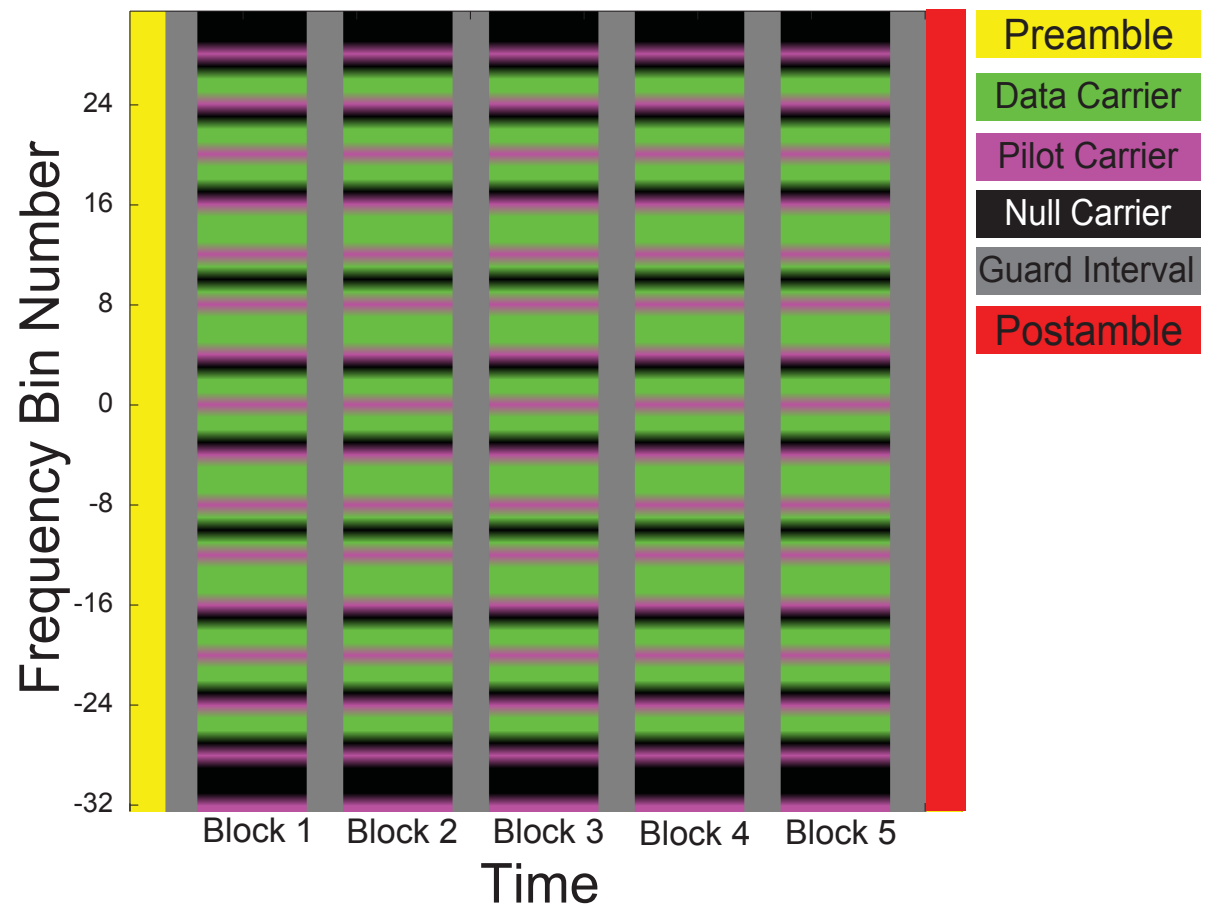

Figure 5: An OFDM data packet. 

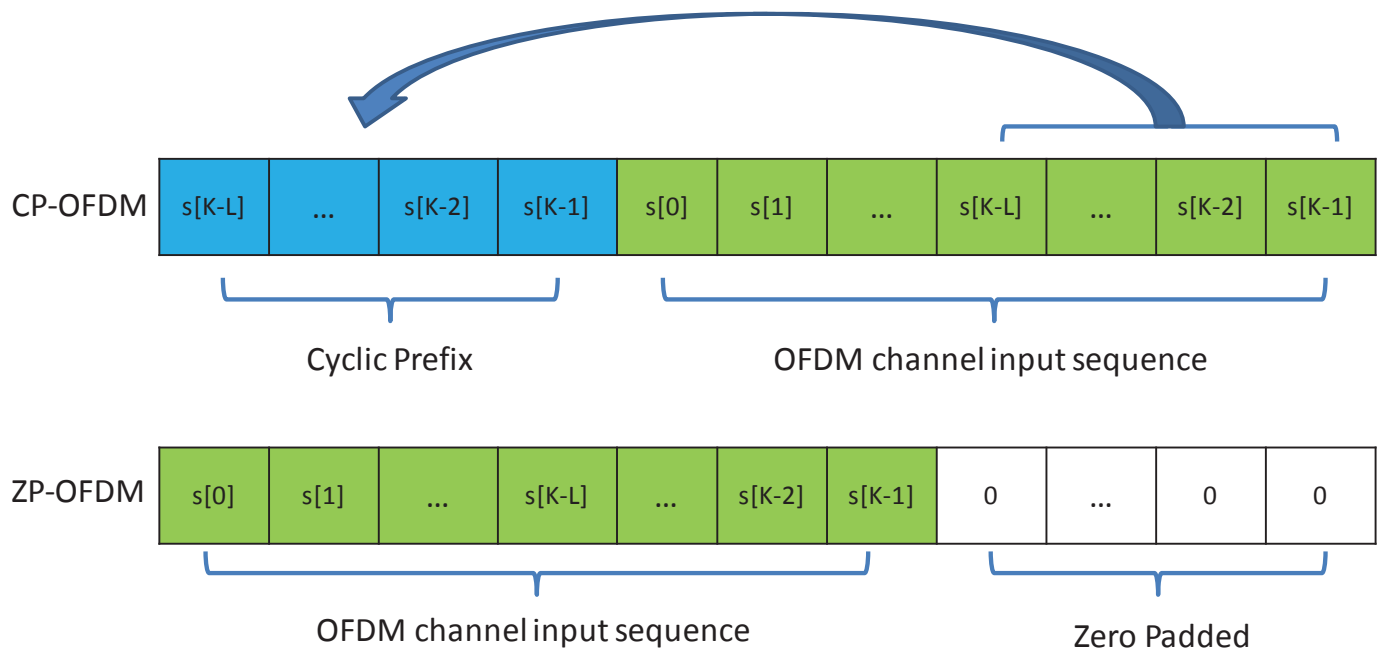

Figure 6: CP-OFDM and ZP-OFDM transmitter processing.

corresponding to the OFDM data block are processed via Fourier transform.

In ZP-OFDM, the guard interval occurs after the data block and is filled with zeros. In contrast to the CP-OFDM receiver, the ZP-OFDM receiver retains the samples from the guard interval and processes the sequence with a method known as overlap and add. The overlap and add method adds samples from the guard interval to received samples corresponding to the beginning of the OFDM data block and then takes the Fourier transform of the newly formed sequence. Fig. 7 illustrates the difference in the receiver processing between CP-OFDM and ZP-OFDM.

The work in [35] compared the merits of CP-OFDM and ZP-OFDM and found that ZP-OFDM was preferable to CP-OFDM because:

- ZP-OFDM guarantees symbol recovery because linear channel equalization is possible irrespective of the location of channel nulls.

- ZP-OFDM provides more flexibility to pursue a variety of different approaches to channel estimation and equalization. 

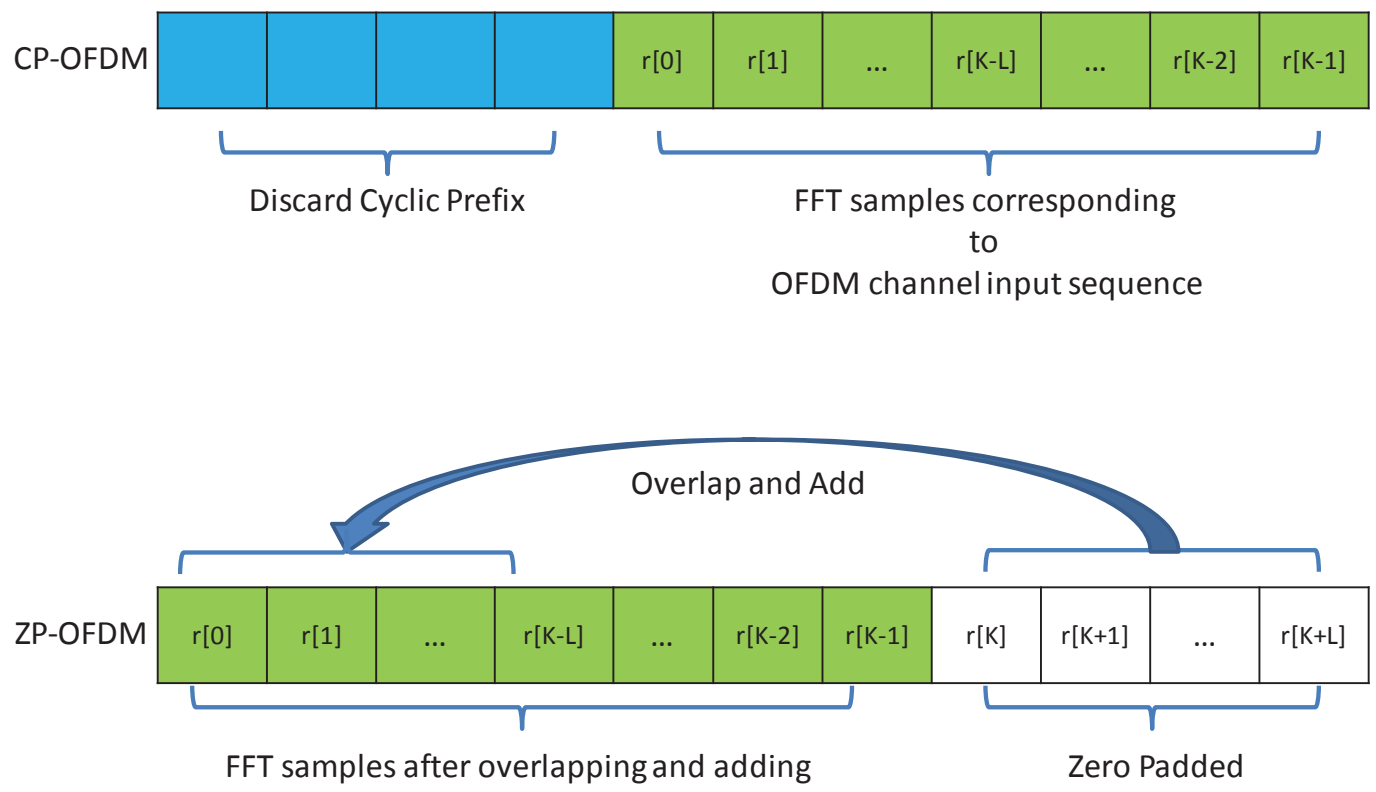

Figure 7: CP-OFDM and ZP-OFDM receiver processing.

- ZP-OFDM with semiblind pilot-based channel estimation tracks channel variations better than CP-OFDM.

According to [35], the one drawback of ZP-OFDM relates to Peak-to-Average power ratio induced clipping. Because ZP-OFDM introduces slightly more nonlinear distortions, it needs slightly more increased power backoff than CP-OFDM. The power backoff issue was addressed in [36] where use of a nonbinary LDPC code in conjunction with ZP-OFDM was found to reduce the Peak-to-Average power ratio. In an interference environment, the retention of guard samples required by the overlap and add processing is an additional drawback for ZP-OFDM. Interference occurring on these samples is retained for ZP-OFDM whereas for CP-OFDM interference occurring in the guard band would be discarded along with the cyclic prefix.

The work that follows uses ZP-OFDM. It was chosen because it is by far the prevailing choice for underwater acoustic communications. Furthermore, the 
interference cancellation technique developed in [17] was applied to ZP-OFDM. Thus, use of ZP-OFDM enables a more direct comparison of this work with that in the literature.

The basic receiver processing chain for a ZP-OFDM signal received on an underwater channel is shown in Fig. 8. The bandpass filter (BPF) removes noise outside the frequency band of interest prior to synchronization of the received signal through the use of the known packet preamble. Doppler scale coarse estimation and resampling removes the main Doppler effect prior to partitioning the packet into blocks. After downshifting to baseband and low pass filtering (LPF), the time domain waveform is transferred to the frequency domain via overlapping, adding and taking the Fast Fourier Transform (FFT). After compensating for any residual Doppler effect (e.g. carrier frequency offset (CFO) estimation and compensation), the channel is estimated and the signal is equalized. Symbol detection and decoding complete the processing steps. Some receivers employ "turbo equalization" if not all of the parity checks are satisfied on the first attempt. In this technique, information from satisfied parity checks is fed back to assist the equalization process where they serve as additional pilot tones. The next sections discusses the mathematics of these operations.

\subsubsection{Transmitted OFDM Signal}

Let $T$ denote the OFDM symbol duration and $T_{g}$ the guard interval between blocks. The OFDM block duration is then $T^{\prime}=T+T_{g}$. The frequency spacing is $\Delta f=1 / T$. The $k$ th subcarrier is at the frequency

$$
\begin{aligned}
f_{k} & =f_{c}+\frac{k}{T} \quad k=\frac{-K}{2}, \ldots, \frac{K}{2}-1 \\
& =f_{c}+k \Delta f
\end{aligned}
$$

where $f_{c}$ is the center frequency and $K$ is assumed even. The signal bandwidth is $B=\frac{K}{T}=K \Delta f$. Let $s[k]$ denote the information symbol on the $k$ th subcarrier 


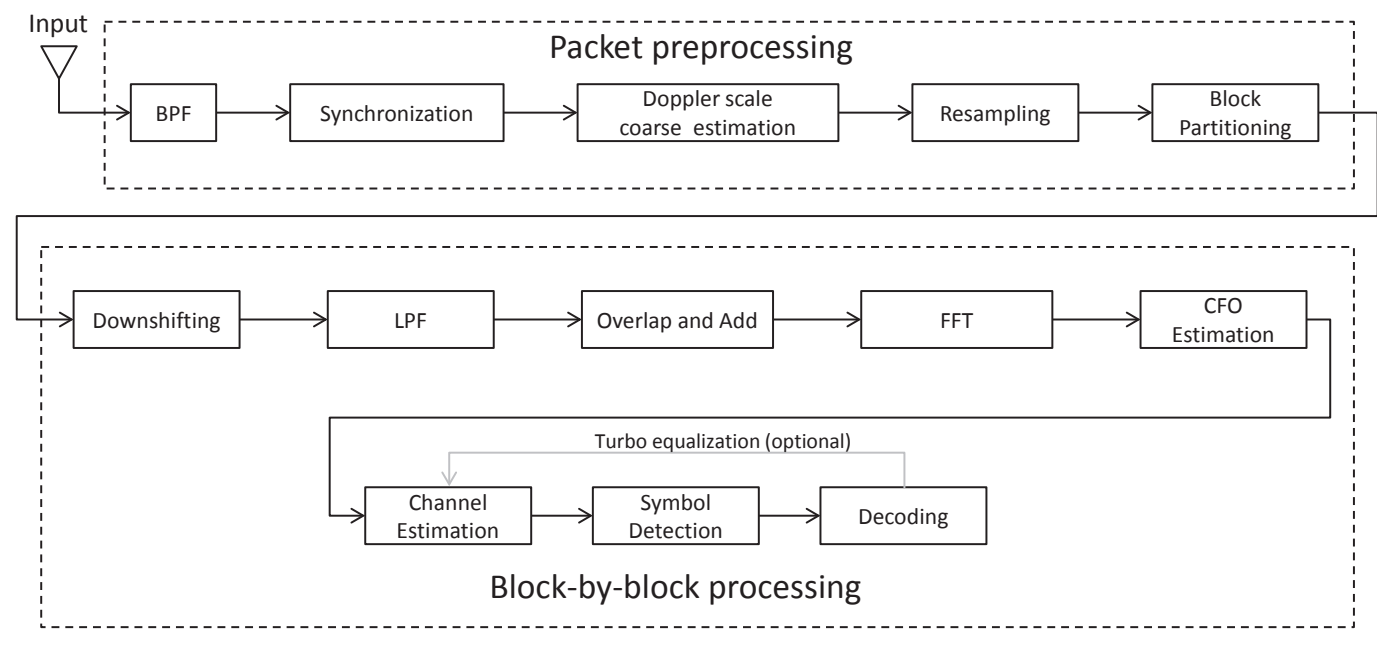

Figure 8: ZP-OFDM receiver for underwater acoustic communications.

and define $\mathcal{S}_{A}$ and $\mathcal{S}_{N}$ as the nonoverlapping sets of active and null subcarriers. The active $\mathcal{S}_{A}$ and null $\mathcal{S}_{N}$ subcarriers satisfy $\mathcal{S}_{A} \cup \mathcal{S}_{N}=\left\{\frac{-K}{2}, \ldots, \frac{K}{2}-1\right\}$. The transmitted bandpass signal is given by

$$
\tilde{s}(t)=2 \operatorname{Re}\left(\sum_{k \in \mathcal{S}_{A}} s[k] e^{j 2 \pi f_{k} t} g(t)\right), \quad t \in\left[0, T^{\prime}\right]
$$

where $g(t)$ is the pulse shaping filter with Fourier transform $G(f)$. In this work, $g(t)=1, t \in[0, T]$ and $g(t)=0$ otherwise. That is, $g(t)$ is a rectangular window of duration $T$.

\subsubsection{Channel Model and Received Signal}

Assuming that the channel exhibits independent Doppler on each path, the channel model of Eq. (21) applies, and the received bandpass signal when no interference is present is given by

$$
\tilde{y}(t)=\sum_{p=1}^{N_{p a}} A_{p} \tilde{s}\left(\left(1+a_{p}\right) t-\tau_{p}\right)+\tilde{n}(t) .
$$

The Doppler effect in $\tilde{y}(t)$ is removed through the two step Doppler compensation technique described in [32]. The main Doppler effect of the received signal 
is removed through resampling using a factor $(1+\hat{a})$ resulting in the resampled signal $\tilde{z}_{\mathrm{rs}}(t)=\tilde{y}\left(\frac{t}{(1+\hat{a})}\right)$. The estimate $\hat{a}$ of the Doppler scaling factor $a$ is found by comparing the time duration of the received packet $\hat{T}_{\mathrm{rx}}$ with the known time duration of the transmitted packet $T_{\mathrm{tx}}$,

$$
\hat{T}_{\mathrm{rx}}=\frac{T_{\mathrm{rx}}}{1+\hat{a}} \Rightarrow \hat{a}=\frac{T_{\mathrm{tx}}}{\hat{T}_{\mathrm{rx}}}-1,
$$

where the received packet time duration is estimated by cross correlating the received signal with the known preamble and postamble. The resampled signal $\tilde{z}_{\mathrm{rs}}(t)$ may contain a residual Doppler error conventionally called the carrier frequency offset (CFO) in RF communications. After bandpass to baseband downshifting of $\tilde{z}_{\mathrm{rs}}(t)$, the carrier frequency offset $\epsilon$ caused by the residual Doppler effect is compensated for by multiplying the baseband signal $z_{\mathrm{rs}}(t)$ by $e^{-j 2 \pi \hat{\epsilon} t}$. The estimate of the carrier frequency offset $\hat{\epsilon}$ is found using the method in [32] which takes the estimate as the frequency $\hat{\epsilon}$ which minimizes the energy on the null carriers in $z(t)=e^{-j 2 \pi \hat{\epsilon} t} z_{\mathrm{rs}}(t)$. After the two step Doppler compensation, the baseband receive signal is given by

$$
z(t)=e^{-j 2 \pi \hat{\epsilon} t} z_{\mathrm{rs}}(t)=e^{-j 2 \pi\left(f_{c}+\hat{\epsilon} t\right)} \tilde{y}\left(\frac{t}{(1+\hat{a})}\right)
$$

and the equivalent Doppler compensated bandpass signal is

$$
\tilde{z}(t)=\tilde{y}\left(\frac{t}{(1+\hat{a})}\right) e^{-j 2 \pi \hat{\epsilon} t} .
$$

Substituting for $\tilde{y}(t)$ from the channel model with path-specific Doppler scales (e.g. Eq. (19) yields

$$
\tilde{z}(t)=\sum_{p=1}^{N_{p a}} A_{p} \tilde{s}\left(\frac{1+a_{p}}{1+\hat{a}} t-\tau_{p}\right) e^{j 2 \pi \hat{\epsilon} t}+\tilde{w}(t),
$$

where

$$
\tilde{w}(t)=\tilde{n}\left(\frac{t}{1+\hat{a}}\right) e^{-j 2 \pi \hat{\epsilon} t}
$$


Taking the Fourier transform of $\tilde{z}(t)$ yields the received signal response as a function of frequency:

$$
\tilde{Z}(f)=\int \sum_{p=1}^{N_{p a}} A_{p} \tilde{s}\left(\frac{1+a_{p}}{1+\hat{a}} t-\bar{\tau}_{p}\right) e^{j 2 \pi \hat{\epsilon} t} e^{-j 2 \pi f t} d t+\int \tilde{w}(t) e^{-j 2 \pi f t} d t
$$

Applying the Fourier transform pairs

$$
\begin{array}{lll}
s(t / T) & \leftrightarrow T S(f T) & \text { Scaling of a variable, } T>0 \\
s\left(t-t_{0}\right) & \leftrightarrow S(f) e^{-j 2 \pi f t_{0}} & \text { Time translation } \\
s(t) e^{j 2 \pi f_{0} t} & \leftrightarrow S\left(f-f_{0}\right) & \text { Frequency translation }
\end{array}
$$

to $s\left(\left(t-t_{0}\right) / T\right) e^{j 2 \pi f_{0} t}$ yields

$$
s\left(\left(t-t_{0}\right) / T\right) \leftrightarrow T S\left(\left(f-f_{0}\right) T\right) e^{-2 \pi f t_{0}}
$$

Thus,

$$
\tilde{s}\left(\frac{1+a_{p}}{1+\hat{a}} t-\tau_{p}\right) e^{-j 2 \pi f \hat{\epsilon}} \leftrightarrow\left(\frac{1+\hat{a}}{1+a_{p}}\right) \tilde{S}\left((f+\hat{\epsilon}) \frac{1+\hat{a}}{1+a_{p}}\right) e^{-j 2 \pi f\left(\frac{1+\hat{a}}{1+a_{p}}\right) \bar{\tau}_{p}}
$$

and letting $b_{p}=\left(a_{p}-\hat{a}\right) /(1+\hat{a})$ so that $1 /\left(1+b_{p}\right)=(1+\hat{a}) /\left(1+a_{p}\right)$ produces

$$
\tilde{Z}(f)=\sum_{p=1}^{N_{p a}} \frac{A_{p}}{1+b_{p}} \tilde{S}\left(\frac{f+\hat{\epsilon}}{1+b_{p}}\right) e^{-j 2 \pi\left(\frac{f+\hat{\epsilon}}{1+b_{p}}\right) \bar{\tau}_{p}}+w(f) .
$$

The OFDM frequency measurement on the $m$ th subcarrier is $z[m]$. Let $z[m]=$ $\left.\tilde{Z}(f)\right|_{f=f_{m}}$ and substitute

$$
\tilde{S}(f)=\sum_{k \in \mathcal{S}_{A}} s[k] G\left(f-f_{k}\right)
$$

into Eq. (35) to yield the frequency domain sample on the $m$ th subcarrier

$$
z[m]=\sum_{p=1}^{N_{p a}} \frac{A_{p}}{1+b_{p}} \sum_{k \in \mathcal{S}_{A}} s[k] G\left(\frac{f_{m}+\hat{\epsilon}}{1+b_{p}}-f_{k}\right) e^{-j 2 \pi\left(\frac{f_{m}+\hat{\epsilon}}{1+b_{p}}\right) \bar{\tau}_{p}}+w[m] .
$$

Reordering the summations produces

$$
z[m]=\sum_{k \in \mathcal{S}_{A}} s[k] \sum_{p=1}^{N_{p a}} \frac{A_{p}}{1+b_{p}} G\left(\frac{f_{m}+\hat{\epsilon}}{1+b_{p}}-f_{k}\right) e^{-j 2 \pi\left(\frac{f_{m}+\hat{\epsilon}}{1+b_{p}}\right) \bar{\tau}_{p}}+w[m] .
$$


Noting that $f_{m}=f_{c}+m / T$ produces

$$
\begin{aligned}
z[m] & =\sum_{k \in \mathcal{S}_{A}} s[k] \sum_{p=1}^{N_{p a}} \frac{A_{p}}{1+b_{p}} G\left(\frac{f_{m}+\hat{\epsilon}}{1+b_{p}}-f_{k}\right) e^{-j 2 \pi\left(\frac{f_{c}+\frac{m}{t}+\hat{\epsilon}}{1+b_{p}}\right) \bar{\tau}_{p}}+w[m] \\
& =\sum_{k \in \mathcal{S}_{A}} s[k] \sum_{p=1}^{N_{p a}} \frac{A_{p}}{1+b_{p}} G\left(\frac{f_{m}+\hat{\epsilon}}{1+b_{p}}-f_{k}\right) e^{-j 2 \pi\left(f_{c}+\hat{\epsilon}\right) \frac{\bar{\tau}_{p}}{1+b_{p}}} e^{-j 2 \pi\left(\frac{m}{T}\right) \frac{\bar{\tau}_{p}}{1+b_{p}}}+w[m]
\end{aligned}
$$

and letting

$$
\tau_{p}=\frac{\bar{\tau}_{p}}{1+b_{p}} \text { and } \xi_{p}=\frac{A_{p}}{1+b_{p}} e^{-j 2 \pi\left(f_{c}+\hat{\epsilon}\right) \tau_{p}}
$$

yields

$$
z[m]=\sum_{k \in \mathcal{S}_{A}} s[k] \sum_{p=1}^{N_{p a}} \xi_{p} e^{-j 2 \pi \frac{m}{T} \tau_{p}} G\left(\frac{f_{m}+\hat{\epsilon}}{1+b_{p}}-f_{k}\right)+w[m] .
$$

Defining the channel mixing matrix,

$$
H[m, k]=\sum_{p=1}^{N_{p a}} \xi_{p} e^{-j 2 \pi \frac{m}{T} \tau_{p}} G\left(\frac{f_{m}+\hat{\epsilon}}{1+b_{p}}-f_{k}\right),
$$

produces the result,

$$
z[m]=\sum_{k \in \mathcal{S}_{A}} H[m, k] s[k]+w[m],
$$

which can be written in vector-matrix notation as

$$
\begin{aligned}
& \underbrace{\left[\begin{array}{c}
z\left[-\frac{K}{2}\right] \\
\vdots \\
z\left[\frac{K}{2}-1\right]
\end{array}\right]}_{:=\mathbf{z}}= \underbrace{\left[\begin{array}{ccc}
H\left[-\frac{K}{2},-\frac{K}{2}\right] & \ldots & H\left[-\frac{K}{2}, \frac{K}{2}-1\right] \\
\vdots & \ddots & \vdots \\
H\left[\frac{K}{2}-1,-\frac{K}{2}\right] & \ldots & H\left[\frac{K}{2}-1, \frac{K}{2}-1\right]
\end{array}\right]}_{:=\mathbf{H}} \underbrace{\left[\begin{array}{c}
s\left[-\frac{K}{2}\right] \\
\vdots \\
s\left[\frac{K}{2}-1\right]
\end{array}\right]}_{:=\mathbf{s}} \\
&+\underbrace{\left[\begin{array}{c}
w\left[-\frac{K}{2}\right] \\
\vdots \\
w\left[\frac{K}{2}-1\right]
\end{array}\right]}_{:=\mathbf{w}}
\end{aligned}
$$

and succinctly written as

$$
\mathbf{z}=\mathbf{H s}+\mathbf{w} .
$$


The channel mixing matrix, $\mathbf{H}$, is specified by $N_{p a}$ triplets $\left\{\xi_{p}, \tau_{p}, b_{p}\right\}$ and may be written as

$$
\mathbf{H}=\sum_{p=1}^{N_{p a}} \xi_{p} \Lambda \boldsymbol{\Gamma}
$$

where $\boldsymbol{\Lambda}(\tau)$ is a $K \times K$ diagonal matrix with the $m$ th diagonal entry,

$$
[\Lambda(\tau)]_{m, m}=e^{-j 2 \pi \frac{m}{T} \tau}
$$

and $\boldsymbol{\Gamma}(b, \epsilon)$ is a $K \times K$ matrix with the $(m, k)$ th entry,

$$
[\boldsymbol{\Gamma}(b, \epsilon)]_{m, k}=G\left(\frac{f_{m}+\epsilon}{1+b}-f_{k}\right) .
$$

The channel mixing matrix $\mathbf{H}$ is typically a banded matrix with the off-diagonal terms equal to zero for diagonal $d>D$ for some $D$. In situations with little Doppler or where the Doppler compensation technique can eliminate its effect, $\mathbf{H}$ is well approximated by its main diagonal and equalization may be adequately performed by assuming a channel mixing matrix with a single main diagonal. This is the assumption of the equalizer in [32]. The off-diagonal terms in $\mathbf{H}$ model inter-carrier interference; that is, the terms $H[m, k]$ where $m \neq k$ describe the

contribution to the measurement on carrier $m$ due to the symbol $s[k]$ transmitted on carrier $k$. When the inter-carrier interference is more substantial, the equalizer must account for it as, for example, in the equalizer of [37] which progressively increases the number of diagonals in $\mathbf{H}$ considered in the equalization process.

\subsection{Survey of Recent Work in OFDM and Interference Mitigation}

Acoustic communications have historically used a single carrier frequency. However, within the last decade research has focused on multi-carrier modulation and OFDM in particular, (see $[32,33,36-59]$ and references therein). OFDM di- 
vides the doubly (time- and frequency-) selective underwater channel into multiple orthogonal subbands. The bandwidth of each subband is chosen to be less than the coherence bandwidth of the channel so that each subband experiences relatively flat fading, thereby simplifying the channel equalization problem. Additionally, Inter-Symbol Interference (ISI) is mitigated since the transmission scheme may be viewed as transmitting many parallel low-rate narrowband signals rather than one fast-rate wideband signal [31]. The effects of ISI may be further reduced by inserting a guard interval between OFDM symbols that is filled with either a cyclic prefix (CP-OFDM) or a zero prefix (ZP-OFDM). Trade-offs between the two prefix approaches are discussed in [35].

Although OFDM mitigates ISI, inter-carrier interference (ICI) resulting from Doppler induced shifting of signals from one subband into another can severely degrade performance. Motion-induced Doppler distortion presents a major obstacle in applying OFDM to underwater channels since both the channel impulse response and the non-uniform effects of relative motion on subbands must be estimated. Solutions to this problem are found in two broad approaches [16]: 1) adaptive synchronization, which requires few subbands dedicated to channel estimation but relies on coherence between adjacent OFDM blocks, and 2) non-adaptive synchronization, which does not rely on channel coherence but requires null subcarriers to gain robustness to fast channel variations. Selection and implementation of an appropriate technique depends on the coherence time and coherence bandwidth of the channel as well as making appropriate trade-offs among computational complexity, required channel estimation accuracy, and data rate. Distributing pilot tones evenly throughout the band and performing interpolation is more efficient than periodically dedicating an entire OFDM symbol to channel estimation [60]. Iterating the equalization and decoding tasks, that is performing turbo equalization, can 
yield large performance improvements [61], and reduced-complexity approximate algorithms are available as in [62] for example.

Through appropriate Doppler compensation of the received signal and judicious assignment of codewords to data slots within an OFDM packet, explicit time and frequency diversity can be achieved. Assigning parts of a codeword to subbands separated by more than the coherence bandwidth of the channel achieves frequency diversity while using an interleaving depth greater than the coherence time of the channel provides time diversity. Experimental results with OFDM have achieved data rates on the order of tens of kilobits per second but none of the algorithms have attempted to account for interference while taking advantage of spatial diversity. The algorithms in [17] and [38] address the possibility of interference but do not provide spatial diversity while the systems developed in [22] achieve time, frequency, and spatial diversity for a vertical line array but do not address interference. The asynchronous multiuser OFDM algorithm proposed in [63] views interfering users as a single external interference which the algorithm parameterizes and uses in an iterative channel estimation, data detection and interference mitigation scheme on a single receiver. Observations from past field experiments indicate that significant improvement in the reliability of message reception can be realized by mitigating interference. Discussion of the interference typical in the underwater environment is available in [64] and [65] .

Interference mitigation has a long history in RF communications but the interference is typically impulsive or narrowband [66] and the algorithms are typically developed for a single receiver. Partial band interference is not addressed [17]. The interference mitigation techniques typically exploit the short time or limited frequency span of the interfering signal. Examples of impulsive noise suppression techniques for multi-carrier modulation may be found in [67-76] while [77-86] ad- 
dress narrowband interference mitigation. Early approaches tended to separate channel estimation and interference detection, and more recent work has focused on jointly estimating the channel and mitigating interference.

Optimum reception is possible given the probability density function of the impulsive noise, but typically the impulsive noise statistics are not available to the receiver [68]. Without a priori knowledge of the impulsive noise statistics, the traditional approach to dealing with impulsive noise has been to mark those symbols hit by impulses as erasures rather than correcting them directly since more erasures than errors can be corrected by the same code [68]. The marking of erasures and error-and-erasure decoding have traditionally been performed separately in cascade [68]. The work in [67] applied the traditional cascade approach but explored using error correction coding (soft error protection) and adaptively adjusting the erasures marking threshold (hard error protection) to combat short time duration, high magnitude interference. Supplying channel state information to the decoder enhanced the performance of the applied forward error correction (FEC) code. The erasures marking threshold was adjusted on each of the multicarrier subchannels to protect those subchannels most susceptible to interference. The authors found that combining both the soft and hard error protection was most effective. A low computational complexity algorithm to cancel the impact of impulse noise in the time domain was proposed in [72]. It employed threshold blanking to cancel the impact of impulse noise on the traditional time domain least squares OFDM channel estimate. In [69], a frequency domain strategy to compensate for impulsive noise after OFDM demodulation and channel equalization was proposed as an alternative to the more conventional approach of suppressing the impulsive noise in the time domain. A support vector machine (SVM) algorithm for coherent robust demodulation of OFDM in the presence of impulse noise interfering with the pilot 
tones was developed in [71]. Compressed sensing techniques were applied to the impulse noise cancellation problem in [73]. Recently, [76] explored bandpass and baseband clipping approaches for suppressing impulsive noise in Direct Sequence Spread Spectrum Systems in underwater communications.

A narrowband interference canceller was developed in [77] by using a model of the power spectral density of the narrowband interferer as prior information. The canceller estimates the linear minimum mean-square error of the spectral leakage by measuring the narrowband interference on a few active or null OFDM subcarriers. [78] and [79] propose mitigating narrowband interference in OFDM through successive cancellation by applying hard or soft decisions based on the known or estimated second order statistics of the received signal. Using the structure provided by FEC coding, each subcarrier is used to predict the narrowband interference contribution on the considered subcarrier in order to subtract out the contribution in an iterative fashion. The most reliable subcarriers are decoded first.

Joint approaches to equalization and interference mitigation may work iteratively such as in [70] or by expanding the states of the decoding algorithm as in [81] and [83]. In [70] applying a clipping and nulling technique at the front of an iterative algorithm significantly reduced the symbol error rate (SER). The iterative decoder further improved performance through a novel syndrome decoding technique which segregated the decoding based on the noise level of the received components. The joint approach to erasure marking and decoding (joint erasure marking Viterbi algorithm (JEVA)) proposed in [68] was motivated by the observation that separation of erasure marking and channel decoding leads to less accurate detection of the impulse noise corrupted symbols because the erasure marker does not take the code structure into account. For a given number of erasures, the JEVA automatically finds the most likely erasure pattern based on 
the most likely trellis path under the erasure constraint. The algorithm effectively mitigates impulsive noise at the expense of higher complexity. In [83], a simple pilot aided interference detector removes in-band interference. Residual interference that cannot be detected by the pilot aided interference detector is removed by a joint interference detection and decoding scheme. By exploiting the code structure in interference detection, the joint scheme detects most of the symbols suffering residual interference without requiring knowledge of the interference distribution.

A message-passing approach to jointly estimate the channel and mitigate strong co-channel interference of similar form as the desired signal was proposed in [87]. The algorithm, based on belief propagation (BP), which performs statistical inference on graphical models by propagating locally computed beliefs, effectively exploited the non-Gaussian statistics of the interference enabling its detection and suppression without requiring specific knowledge of the interfering signal. Two blind algorithms to mitigate multiple interferers were proposed in [88]: one for the case of strong signal and one for the case of strong interference. Both algorithms find the coefficients of the linear Minimum Mean Square Error filter based on the autocorrelation matrix function of the channel outputs.

Generally, joint approaches provide better performance at the cost of additional computational complexity. Limitations on system performance may be found in [89] for OFDM systems subject to impulsive noise and for multicarrier and single carrier quadrature amplitude modulation (QAM) systems in [90]. The capability of Low Density Parity Check (LDPC) and turbo coding to mitigate burst errors is discussed in [91].

In mobile radio, optimal receiver combining (ORC) [92] is a multi-receiver technique that has been used for years to combat interference. Its performance bounds were developed in [93], but the channel is much less complicated than in 
underwater communications: it contains no multipath and Doppler effects may be safely ignored. The ORC algorithm assumed the channel was known and the combining was performed on the time domain received waveform. Its original application was to Phase-Shift Keying (PSK) modulation.

Some more recent work has addressed diversity combining in the underwater environment. A receiver that addressed the challenges of the underwater channel and performed joint diversity combining of multiple channel receptions with channel equalization was proposed in [94]. More recently, [95] proposed a multiple-input multiple output system which used space-time coding and iterative decoding techniques to obtain high data rates over shallow-water, medium range channels in the absence of interference. In the 200-300 Hz frequency range, [96] exploited beam diversity among sensors separated by hundreds of meters to improve communication performance.

Whereas processing time and computational complexity are critical drivers in many RF applications (such as mobile phones, digital audio and video broadcasting), underwater communication applications exist (such as submarine to shore message trafficking) where decoding delays are tolerable and vast amounts of computational power are available. Thus, the additional available decoding time and computing power may be employed to untangle the more complicated channel and Doppler effects.

\subsection{Motivation for the Research}

The motivation for this research is to examine approaches to leverage the spatial diversity of underwater acoustic communications networks suffering from interference. The AUTEC network [21] consisting of 96 fiber-optically connected hydrophones that receive signals over a time-varying multipath channel in the presence of interference provides the impetus to examine a unique communica- 
tions problem. Currently, approximately 97 percent of messages are correctly decoded. Of the remaining three percent, many suffer from partial-band interference emanating from multiple spatially separated sources. The nature of the interference is different from the impulsive or narrowband interference typically encountered in other applications. Some of the interfering waveform parameters such as bandwidth or time duration are known a priori or can be determined in situ. Furthermore, unlike RF communications and acoustic array processing applications where interference is highly correlated in time among the various receivers, in the acoustic network interference affects different portions of the received signals due to the wide separation of the receivers and the low speed of propagation. The degradation in the received signal is highly variable, depending on the relative position of the interferers, information source and receivers as well as the channel conditions. The spatial diversity implicit in the network offers the opportunity to mitigate interference, but the underwater acoustic channel presents challenges that must be addressed, namely: severely limited operational bandwidth requiring explicit Doppler compensation for multicarrier systems; significant multipath spread and non-stationary statistics which complicate channel estimation and equalization; and the need for explicit time synchronization. An example of the technical problems this research addresses is shown in Fig. 9. Here, two received OFDM data blocks suffer from interference caused by a linear frequency modulated (LFM) waveform. Note that the interference corrupts first half of the received signal on channel A and the second half of the signal on channel B. Fig. 10 shows the signal constellation resulting from processing the receptions on each channel individually, using the conventional maximum ratio combining (MRC) approach and the spatial diversity reconstruction technique developed by this research. Taking advantage of multiple receivers and the time orthogonality 

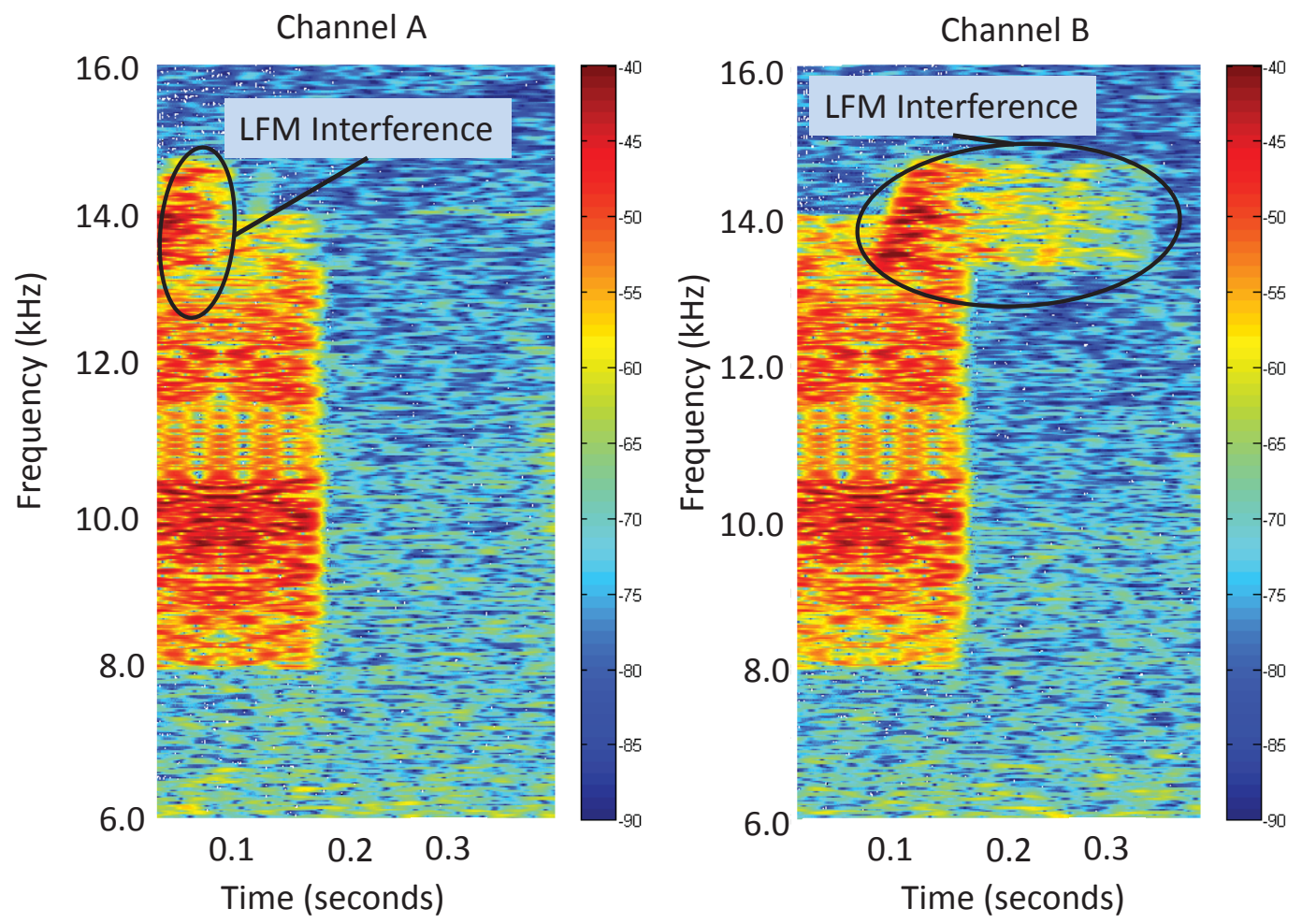

Figure 9: Two received OFDM data blocks suffering from interference which corrupts the receptions at different times.

of the interference reduces the error rate. Reducing the message error rate to less than one percent will enhance safety on the AUTEC range and enable more users to operate on the range simultaneously. The AUTEC range is currently an overbooked Navy resource. Significant scheduling flexibility and cost reduction can be realized by developing the capability to communicate effectively in this congested acoustic environment.

The application of this work extends beyond the AUTEC network to cabled acoustic networks in general and other situations where interference corrupts receptions on widely separated receivers. As we continue to explore and operate in the oceans, the number of activities grows. Many of these activities, such as oil exploration and mapping of the ocean floor using active sonar, generate signals of interest to the user of the application at hand but create interference from 

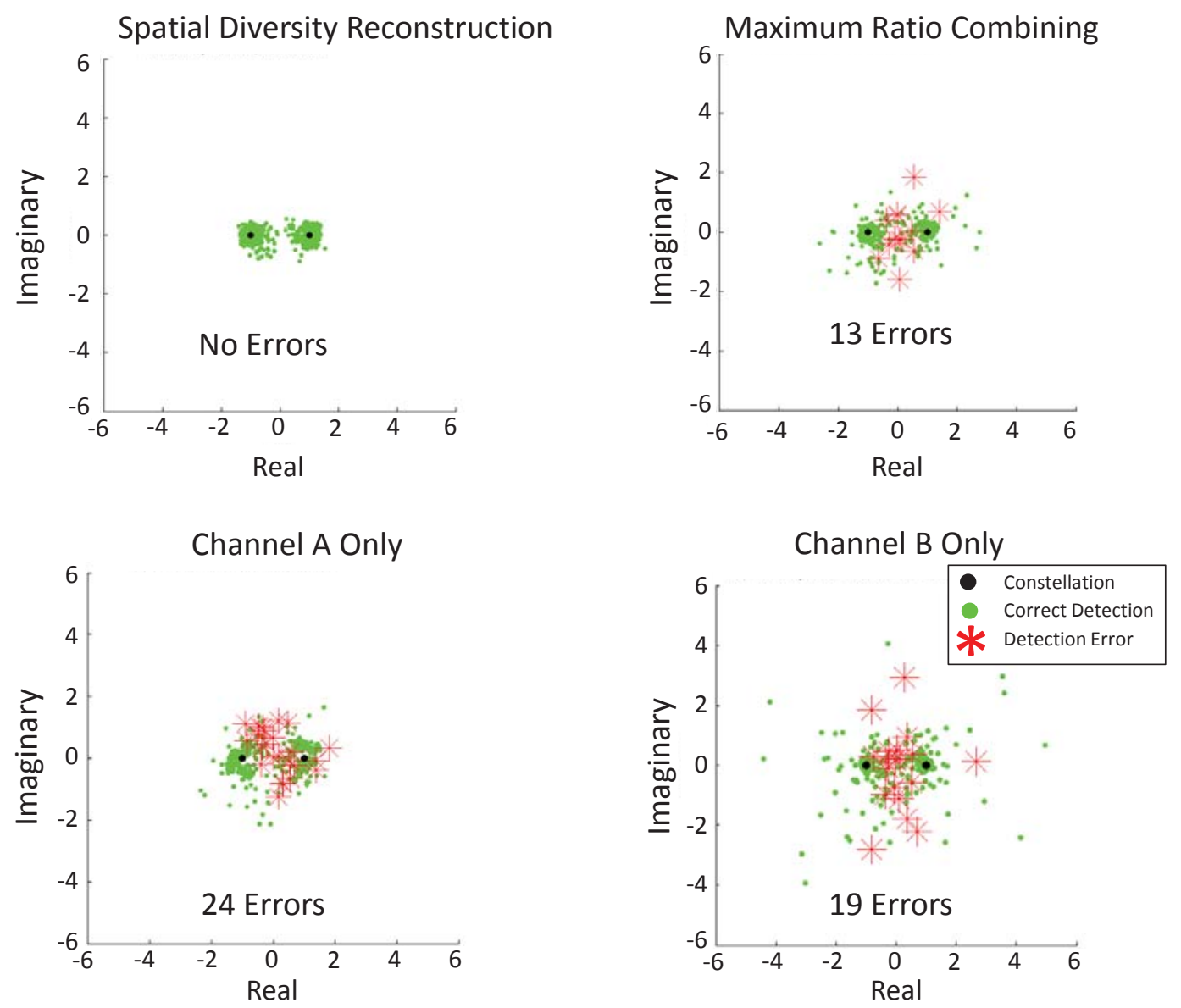

Figure 10: Received signal constellations resulting from processing the receptions in Fig. 9 using different combining techniques.

The black dots indicate the transmitted Binary Phase Shift Keying (BPSK) symbol constellation. The green dots indicate correctly detected symbols and the red asterisks indicate detection errors. Top left pane: combining using the spatial diversity reconstruction technique developed by this research. Top right pane: combining using the conventional maximum ratio combining technique. Lower left and right panes: processing channel $\mathrm{A}$ and $\mathrm{B}$ independently. 
the perspective of other users. Communications is an essential aspect of many of these operations. For example, autonomous undersea vehicles require a reliable communications link to send and receive data to remote users. As these activities increase, understanding the limits interference places on performance and developing approaches to mitigate its effects are important areas of research.

\subsection{Summary}

After providing some background information about the early development of underwater acoustic communications and the AUTEC acoustic network, this chapter surveyed the challenges of the acoustic channel and reviewed recent work in interference mitigation and OFDM as motivation for the development of a spatial diversity receiver that will be introduced in the next chapter. The wave equation governs the propagation of sound underwater, but solving it directly is difficult. Instead, practical underwater communication algorithms are built on the simpler channel models discussed in section 1.3.2. As human activities in the ocean increase, its acoustic environment becomes more congested, motivating the need to develop techniques to mitigate interference and understand the limits interference places on performance. 


\section{CHAPTER 2 \\ Spatial Diversity Receiver}

\subsection{Introduction}

After providing an example of the spatial diversity exhibited at AUTEC, section 2.3 introduces the concept of a spatial diversity receiver. Section 2.4 applies the concept of a spatial diversity receiver to the OFDM modulation scheme and describes an algorithm capable of mitigating partial band, partial block interference in OFDM signals. After describing the approach to using spatial diversity to mitigate interference, the parameterized interference cancellation algorithm from [17] is presented. Section 2.7 compares the approaches of the two algorithms.

\subsection{Spatial Diversity at AUTEC}

Acoustic propagation is much more environmentally variable than $\mathrm{RF}$, largely due to the slow $\sim 1500$ mps speed of propagation and its strong dependence on ocean temperature, pressure and salinity. AUTEC has a downward-refracting acoustic environment with surface water temperature of typically $26^{\circ} \mathrm{C}\left(80^{\circ} \mathrm{F}\right)$ and the thermocline appearing between 120 and 210 meters (400 to 700 feet). The propagation of sound under these environmental conditions exhibits shadows zones. Fig. 11 illustrates this variability from a networking point of view: thirty-nine messages were transmitted from the location marked by the blue star, numbers of receptions at receiver hydrophones are indicated by the number adjacent to each phone, and the relative signal strength and quality, as assessed by the AUTEC ACOMMs processing algorithm, are represented by color. Green circles represent receptions with high signal-to-noise ratio (SNR), red circles indicate correctly-decodable receptions with degraded SNR and/or enhanced or dynamic multipath and black circles represent low SNR receptions that are unusable. (The 
small black dots indicate hydrophones that were not part of the experiment). As expected, receivers close to the transmitter receive the strongest signals, although even in this case the number of high-quality receptions drops off precipitously with range: a receiver less than four miles away only received seven high-quality receptions. The range to some receivers that were unable to decode any transmissions is in many cases comparable to hydrophones with excellent reception. This patterning is very typical of the oceanic area and arises from spatial fluctuations of three-dimensional temperature distribution in the ocean. Correlation of reception intensity or quality between successive packet transmissions is typically low.

The acoustic environment at AUTEC is congested. Active sonars, mammal vocalizations and snapping shrimp are some of the sources that interfere with underwater acoustic communications. Currently 97 percent of the transmitted messages are correctly decoded. Reducing the error rate to below one percent will enhance range safety and enable more platforms to operate simultaneously on the range.

\subsection{A Spatial Diversity Receiver to Mitigate Interference}

The concept of combining multiple receptions of the same transmitted signal is not new. In a network, information is available for combining from multiple layers: 1) the received waveform, 2) the detected received symbols and 3) the decoded information. In general, a diversity processor could operate on any of these layers or among the different layers. This work focuses on combining the received waveforms. The concept of combining received waveforms has been a standard approach for decades. Maximum ratio combining (MRC) weights the received waveforms based on the signal-to-noise ratio at each receiver (see chapter 7, section 5 of [33]). Optimal ratio combining (ORC) [92] also takes advantage of the differential fading received signals experience but additionally considers 


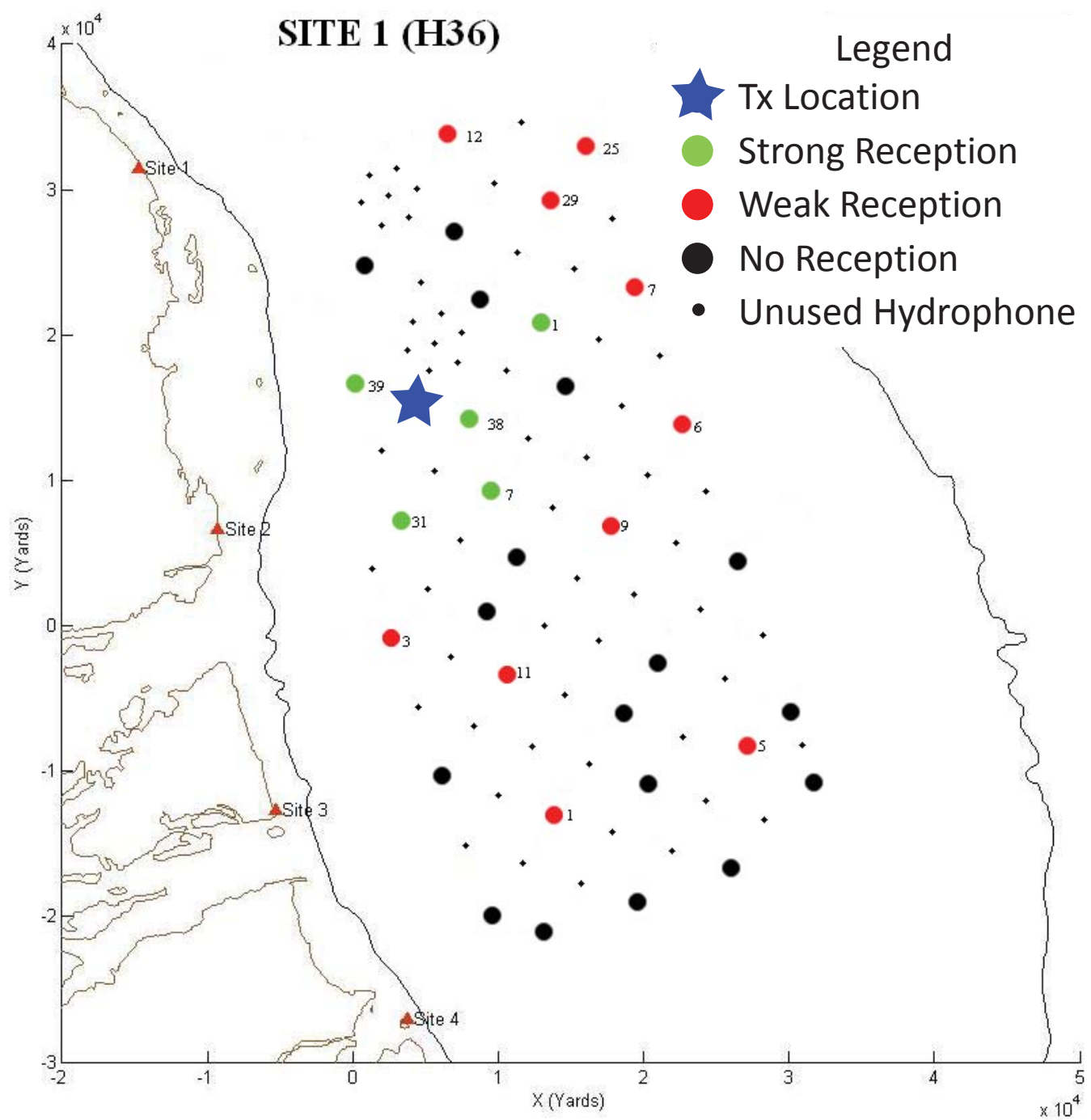

Figure 11: Receptions as a function of geometry from the transmitter. Green circles represent receptions with high SNR, red circles indicate correctly-decodable receptions with degraded SNR and/or enhanced or dynamic multipath and black circles represent low SNR receptions that are unusable. (The small black dots indicate hydrophones that were not part of the experiment.) 
the impact of co-channel interferers in calculating the weighting of the received waveforms. ORC was specifically developed to combat co-channel interference on Rayleigh fading channels for mobile radio. It was originally applied to Phase-ShiftKeying (PSK) modulation and assumed the channel was known. Whereas ORC takes advantage of the differential fading of the virtually synchronously received interference among the receivers, the algorithm described here relies on the slow speed of signal propagation underwater which results in the received interference exhibiting some degree of time orthogonality. Underwater receptions also experience differential fading and when the interference is not time orthogonal, the algorithm makes use of this in a manner similar to ORC.

Receivers that seek to combine information at the time-domain waveform level face some common challenges. Obviously, the combining must occur on waveforms containing the same transmitted message. Once the signals containing the same message have been selected the receiver must:

- Properly time align the received waveforms

- Estimate and equalize the channel through which the waveform was transmitted

- Determine and apply the appropriate weights to the received signal

- Re-equalize the combined waveform

Data detection and decoding can then be performed. Proper synchronization and equalization of the received waveforms are critical steps and errors in these processes lead to performance degradation. The waveform reconstruction algorithm described below assumes that the correct waveforms have been selected for combining and that these waveforms have been properly time aligned. 
In the context of the literature, the algorithm can be seen as building upon ORC in taking explicit advantage of the time orthogonality of the interference. Recalling that the ORC technique was originally applied to PSK modulation assuming the channel was known provides another vantage point. From this perspective, the work extends the ORC concept to OFDM modulation and offers an approach to dealing with channel estimation and equalization that is suitable to OFDM modulation.

\subsection{Spatial Diversity Reconstruction for OFDM}

The OFDM spatial diversity receiver for underwater communications identifies portions of the signal suffering from interference on different receivers, removes these portions of the signal and then optimally combines the remaining clean portions of the signal. The process, illustrated Fig. 12, involves switching between the time and frequency domain because interference excision and waveform recombining must be done on equalized time-domain waveforms and all of the information for channel estimation is present in the frequency (or OFDM symbol) domain. Pre-processing synchronizes the received signals and adjusts for Doppler induced dilation or contraction. The signals are then transferred to the frequency domain for removal of any residual Doppler effect, channel equalization and testing for the presence of interference. The frequency domain interference detector compares the signal power on the subcarriers inside and outside of the interference band to determine if interference is present. If interference is declared on any signal, the group of signals is transferred back to the time domain. In the time domain, knowledge of the time duration of the interference aids in determining which portion of the time domain waveform suffers from interference. Reconstruction of the time domain waveform excises time orthogonal interference and averages across portions of the waveform suffering from only noise. If the interference is not time orthogo- 


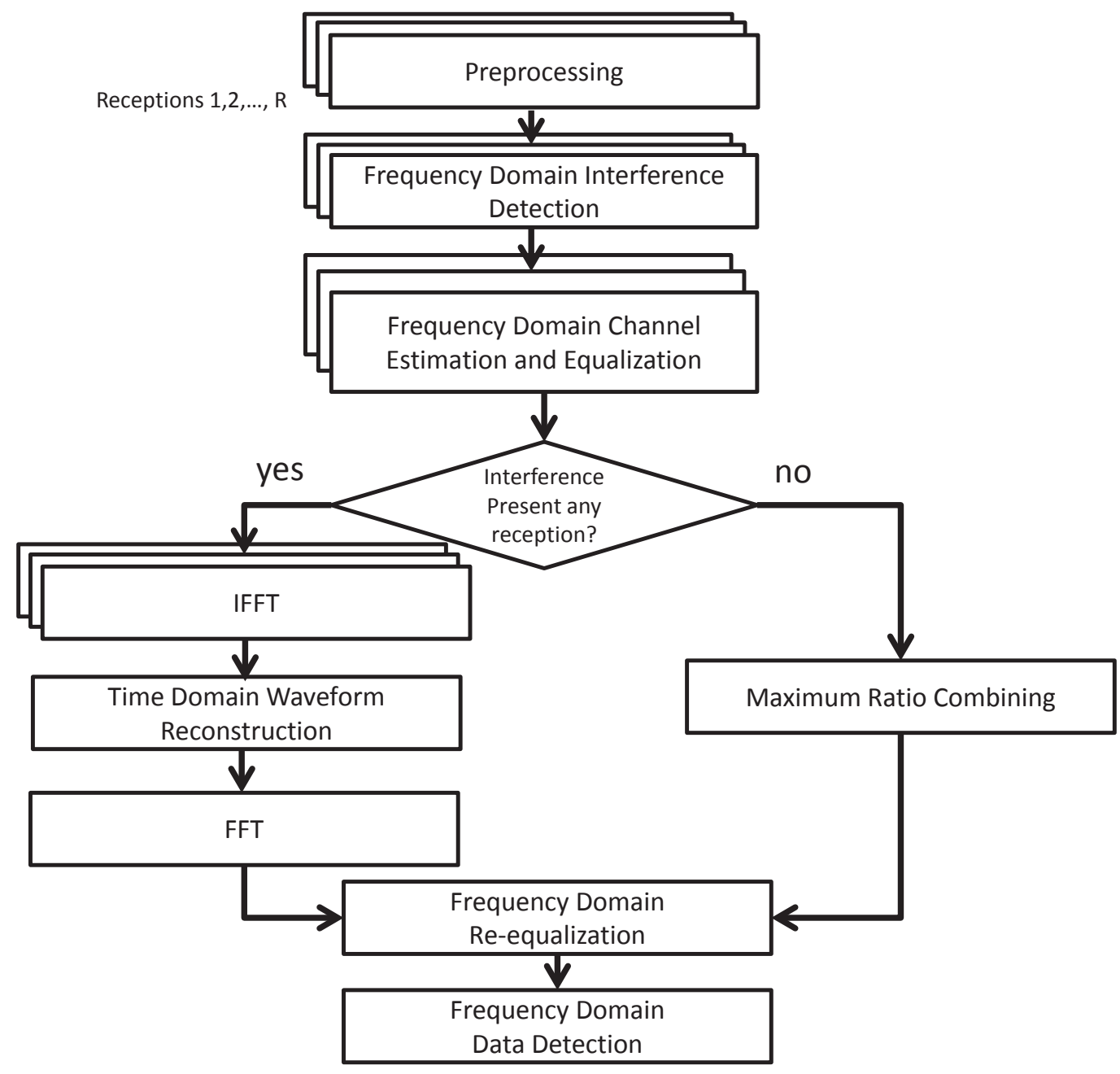

Figure 12: Spatial Diversity Reconstruction (SDR) processing.

nal (e.g. it occupies the same time bins on all receivers), averaging is performed. Fig. 13 illustrates the combining strategy for the case of three receivers where the time domain samples suffering from interference are indicated in red and samples contaminated by only noise are indicated in green. The synthesized signal is then transferred back to the frequency domain for re-equalization and data detection.

At the input to the SDR processing the received baseband time domain signal is given by

$$
z(t)=y\left(\frac{t}{1+\hat{a}}\right) e^{j 2 \pi \hat{\epsilon} t},
$$

and its frequency domain samples on each of the $K$ subcarriers are contained in 


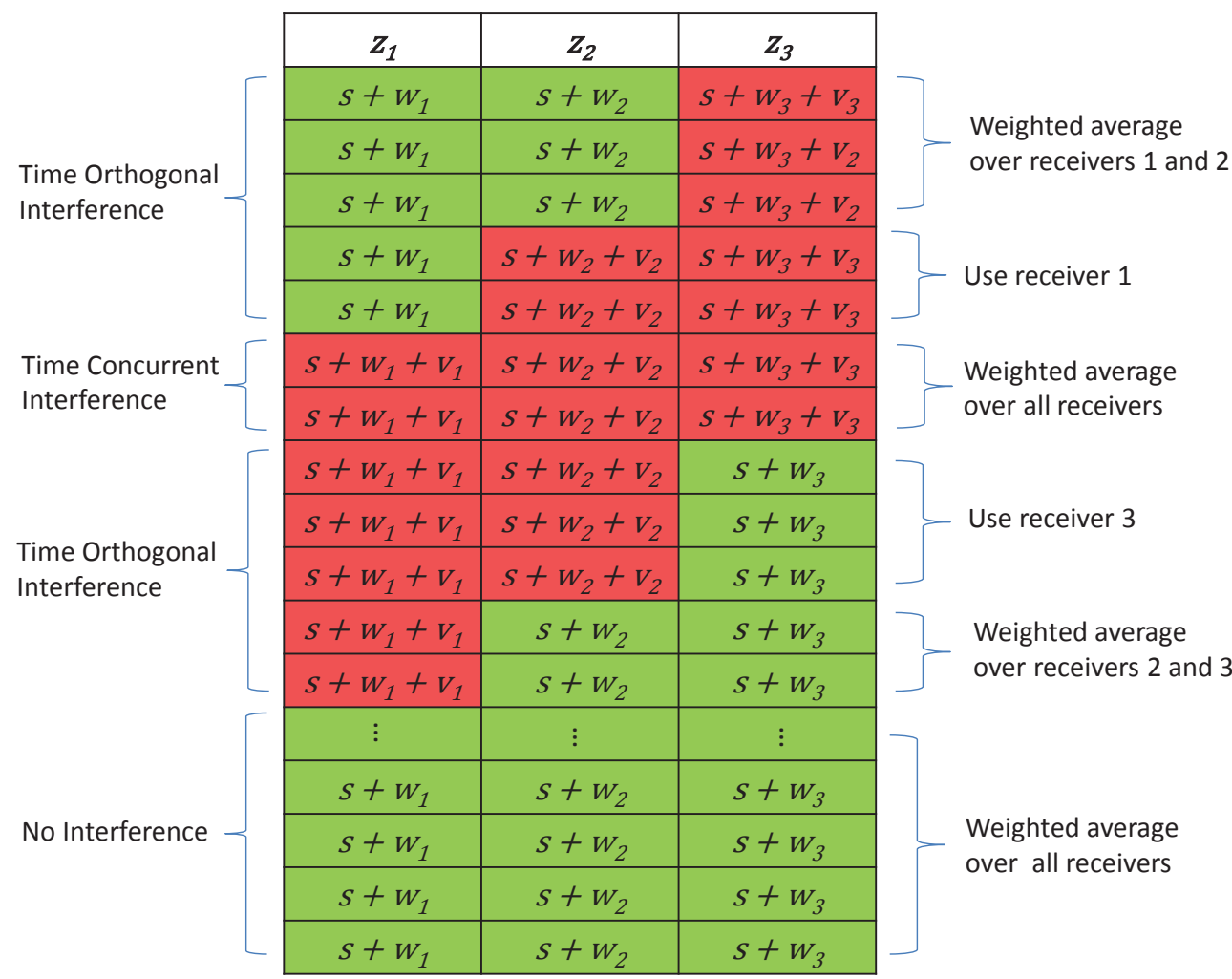

Figure 13: Illustration of the combining strategy for the case of three receivers.

Time domain samples suffering from interference are indicated in red and samples contaminated by only noise are indicated in green. Reconstruction of the time domain waveform excises time orthogonal interference and averages across portions of the waveform suffering from only noise. If the interference is not time orthogonal (e.g. it occupies the same time bins on all receivers), averaging is performed. 
the vector

$$
\mathbf{z}=\mathbf{H s}+\mathbf{w}+\mathbf{v}
$$

where $\mathbf{H}$ is the channel mixing matrix, $\mathbf{w}$ is the additive noise and $\mathbf{v}$ is the interference. The interference has time duration $T_{I}$ (which contaminates $N_{I}$ baseband samples), center frequency $f_{I_{c}}$ and bandwidth $B_{I}{ }^{1}$. It occupies the frequency band $\mathcal{B}_{I}:=\left[f_{I_{c}}-\frac{B_{I}}{2}, f_{I_{c}}+\frac{B_{I}}{2}\right]$.

\subsubsection{Frequency Domain Interference Detection}

Let $\overline{\mathcal{S}}_{v}$ denote the subcarriers in the noise only band $\left(\overline{\mathcal{S}}_{v}:=\left\{m: f_{m} \notin\right.\right.$ $\left.\mathcal{B}_{I}\right\}$ ), and let $\mathcal{S}_{v}$ denote the subcarriers in the band which potentially suffers from interference $\left(\mathcal{S}_{v}:=\left\{m: f_{m} \in \mathcal{B}_{I}\right\}\right)$. The frequency domain interference detector declares interference if

$$
\frac{1}{\left|\mathcal{S}_{v}\right|} \sum_{k \in \mathcal{S}_{v}}|z[k]|^{2}>\frac{1}{\left|\overline{\mathcal{S}}_{v}\right|} \sum_{k \in \overline{\mathcal{S}}_{v}}|z[k]|^{2}
$$

and the Kolmogorov-Smirnov hypothesis test determines the samples $z\left[k \in \mathcal{S}_{v}\right]$ and $z\left[k \in \overline{\mathcal{S}}_{v}\right]$ to be from different distributions with significance level of greater than a chosen threshold, $k s_{\text {th }}$. The MATLAB ${ }^{\circledR}$ function kstest2 may be used to perform the hypothesis test. Alternatively, a Generalized Log Likelihood Ratio (GRLT) hypothesis test could be constructed similar to the test developed in [17] and discussed in Section 2.6.2. The GRLT interference detector is left for future work.

\footnotetext{
${ }^{1}$ The signal $s(t) \leftrightarrow S(f)$ is time and band limited if

$$
\begin{array}{cc}
|s(t)|<\sigma_{t}^{2} & \text { for } \quad t<t_{0} \text { and } t>t_{1} \\
|S(f)|<\sigma_{f}^{2} & \text { for } \quad f<f_{0} \text { and } f>f_{1} .
\end{array}
$$
}




\subsubsection{Channel Equalization}

Next the channel $\mathbf{H}$ is estimated and equalized. A number of channel estimation and equalization algorithms are available such as [37, 50, 52, 61, 62, 87]. This work used the least squares algorithm from [32] which is applicable provided the number of pilot tones $K_{p}=\left|\mathcal{S}_{P}\right|$ is greater than the length of the channel, e.g. $K_{p} \geq N_{p a}+1$. The $K_{p}$ pilot tones at the subcarrier indices $p_{1}, p_{2}, \ldots, p_{K p}$ i.e. $\left\{s\left[p_{i}\right]\right\}_{i=1}^{K_{p}}$ are known to the receiver and the channel taps can be found based on the least squares (LS) formulation

$$
\begin{aligned}
& \overbrace{\left[\begin{array}{c}
z_{p_{1}} \\
\vdots \\
z_{p_{K_{p}}}
\end{array}\right]}^{\mathbf{z}_{p}}=\overbrace{\left[\begin{array}{c}
w_{p_{1}} \\
\vdots \\
w_{p_{K_{p}}}
\end{array}\right]}^{\mathbf{w}_{p}}+\overbrace{\left[\begin{array}{c}
v_{p_{1}} \\
\vdots \\
v_{p_{K_{p}}}
\end{array}\right]}^{\mathbf{v}_{p}}+\overbrace{\left[\begin{array}{lll}
s\left[p_{1}\right] & & \\
& \ddots & \\
& & \left.\mathbf{D}_{s\left[p_{K_{p}}\right]}\right]
\end{array}\right]}^{\mathbf{D}_{s}} \\
& \times \overbrace{\left[\begin{array}{cccc}
1 & e^{-j \frac{2 \pi}{K} p_{1}} & \cdots & e^{-j \frac{2 \pi}{K} p_{1} L} \\
\vdots & \vdots & \ddots & \vdots \\
1 & e^{-j \frac{2 \pi}{K} p_{1}} & \cdots & e^{-j \frac{2 \pi}{K} p_{K_{p}} L}
\end{array}\right]}^{\mathbf{F}} \overbrace{\left[\begin{array}{c}
h_{0} \\
\vdots \\
h_{L}
\end{array}\right]}^{\mathbf{h}} .
\end{aligned}
$$

Following the design principles in [32], this work spaced unit amplitude pilot tones equally within the $K$ subbands and the equalizer assumed that the channel length was $L$ baseband samples. Because the pilots are equispaced, $\mathbf{F}^{H} \mathbf{F}=K_{p} \mathbf{I}_{L+1}$ and since they are of unit amplitude, $\mathbf{D}_{s}^{H} \mathbf{D}_{s}=\mathbf{I}_{K_{p}}$, where $\mathbf{I}_{M}$ is an $M \times M$ identity matrix. The LS solution for Eq. (51) is

$$
\hat{\mathbf{h}}_{\mathrm{LS}}=\frac{1}{K_{p}} \mathbf{F}^{H} \mathbf{D}_{s}^{H} \mathbf{z}_{p}
$$

The frequency domain estimate of the channel is obtained from $\hat{\mathbf{h}}_{\mathrm{LS}}$ using

$$
H[m]=\sum_{l=0}^{L} h_{l} e^{-j 2 \pi l m / K} .
$$

This equalizer ignores any residual Inter-Carrier Interference remaining after compensating for the Doppler effect. More sophisticated equalization algorithms exist 
which allow for off diagonal terms in the channel matrix $\mathbf{H}$. This simpler algorithm adequately equalized the signals transmitted during the at-sea experiments reported in the next chapter. Since the received signal suffers interference on only some of the received pilot tones, a weighted least squares approach could be reasonably expected to improve equalization performance. However, since the standard LS approach performed adequately on the data, no effort was made to implement a weighted least squares equalizer.

\subsubsection{Time Domain Waveform Reconstruction}

Provided interference is detected on any reception, the received frequency samples are transformed to the time domain, $\boldsymbol{z}=\operatorname{IFFT}(\mathbf{z}, K)$, where boldface italics indicates the time domain signal vector and non-italicized boldface indicates the frequency domain vector. A rolling window of size $N_{I_{\text {win }}}$ sums the energy in the time domain signals. Since the interference of time duration $T_{I}$ occurs in $N_{I}$ samples, the time domain interference window should be chosen of size $N_{I_{\text {win }}} \geq N_{I}$. However, selecting $N_{I_{\text {win }}} \gg N_{I}$ should be avoided because the algorithm removes all the samples in the interference window from the averaging process. Because the frequency domain samples in ZP-OFDM are formed from overlapping and adding samples from the guard period $T_{g}$ with samples in the symbol period $T$, the window "wraps" around $\boldsymbol{z}$ as shown in Fig. 14. There are $N=K$ windows whose output is given by

$$
q[i]=\sum_{n=0}^{N_{I_{\text {win }}}-1}|z[\bmod (i+n, K)]|^{2} .
$$

Time domain interference is declared in the window where $q$ achieves its maximum.

The time domain reconstruction is a weighted combination of the received waveforms which takes into account where the interference occurs as well as the signal, noise and interference power on the receivers. An example of the weighting scheme for three receivers is illustrated in Fig 15. The calculation of the weights 


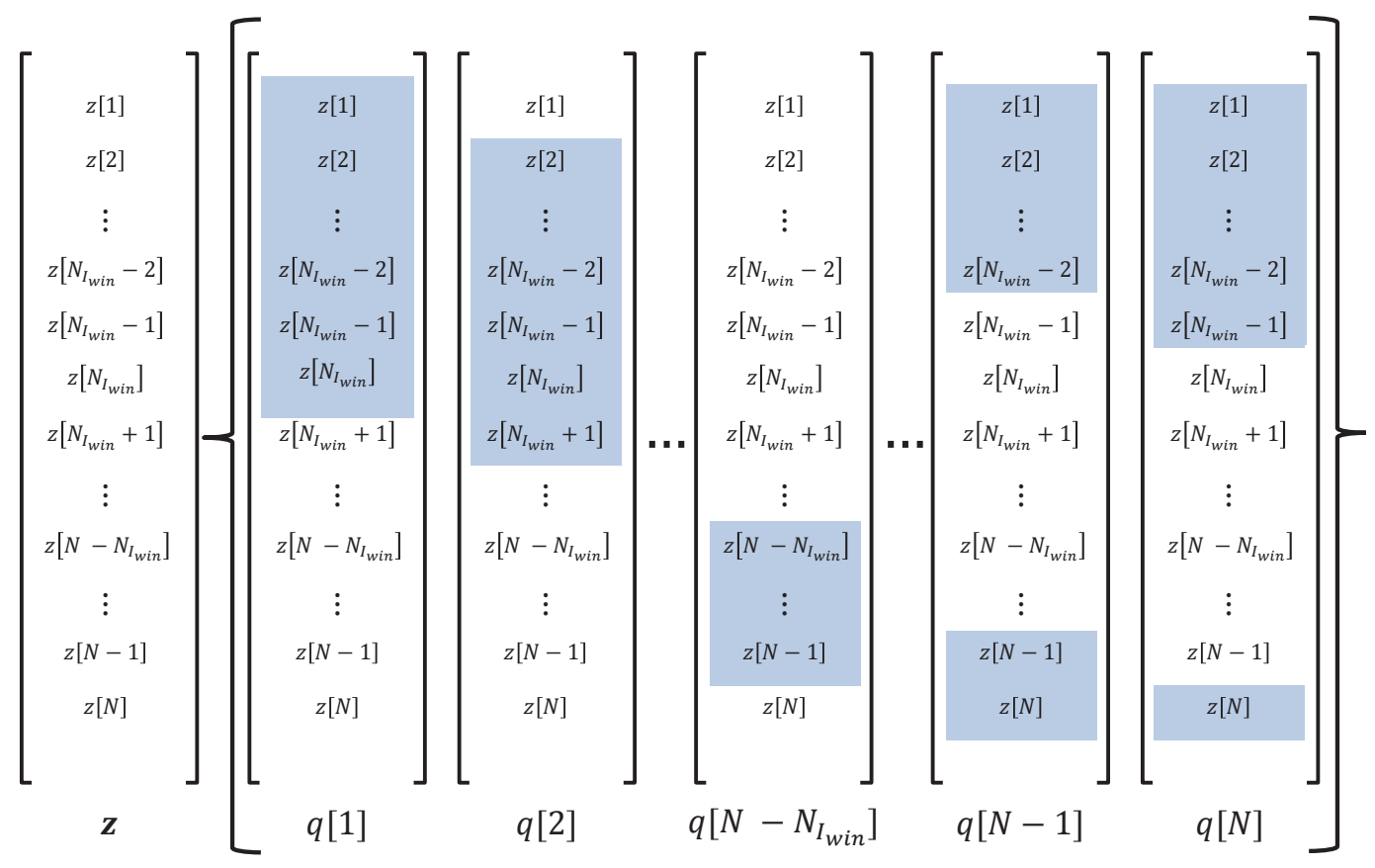

Figure 14: The SDR time domain interference detection windows. The $N=K$ time domain interference detection windows $q[n]$ of size $N_{I_{\text {win }}}$. Blue indicates the samples in each window. The windows "wrap" around the time domain sample vector $\boldsymbol{z}$ due to the overlap and add processing of the ZP-OFDM waveform. 
is described below:

Let $\bar{\alpha}_{r}$ be the ratio of the signal power to noise variance on the $r$ th receiver outside of the interference band calculated as

$$
\bar{\alpha}_{r}=\frac{\hat{\sigma}_{\bar{s}_{r}}^{2}}{\hat{\sigma}_{\overline{\mathcal{B}}_{I_{r}}}^{2}}
$$

where $\hat{\sigma}_{\bar{s}_{r}}^{2}$ and $\hat{\sigma}_{\overline{\mathcal{B}}_{I_{r}}}^{2}$ are estimates of the signal power and noise variance on the $r$ th receiver obtained by averaging over the active and null subcarriers outside of the interference band as follows:

$$
\begin{aligned}
\hat{\sigma}_{\overline{\mathcal{S}}_{r}}^{2} & =\mathbb{E}_{\left\{k \in \mathcal{S}_{A} \cap \overline{\mathcal{S}}_{v}\right\}}\left[\left\|z_{r}[k]\right\|^{2}\right]-\hat{\sigma}_{\overline{\mathcal{B}}_{I_{r}}}^{2} \\
\hat{\sigma}_{\overline{\mathcal{B}}_{I_{r}}}^{2} & =\mathbb{E}_{\left\{k \in \mathcal{S}_{N} \cap \overline{\mathcal{S}}_{v}\right\}}\left[\left\|z_{r}[k]\right\|^{2}\right] .
\end{aligned}
$$

Let $\alpha_{r}$ be similarly defined as $\bar{\alpha}_{r}$ but occurring inside the interference band. Thus,

$$
\alpha_{r}=\frac{\hat{\sigma}_{s_{r}}^{2}}{\hat{\sigma}_{\mathcal{B}_{I_{r}}}^{2}}
$$

and

$$
\begin{aligned}
\hat{\sigma}_{s_{r}}^{2} & =\mathbb{E}_{\left\{k \in \mathcal{S}_{A} \cap \mathcal{S}_{v}\right\}}\left[\left\|z_{r}[k]\right\|^{2}\right]-\hat{\sigma}_{\mathcal{B}_{I_{r}}}^{2} \\
\hat{\sigma}_{\mathcal{B}_{I_{r}}}^{2} & =\mathbb{E}_{\left\{k \in \mathcal{S}_{N} \cap \mathcal{S}_{v}\right\}}\left[\left\|z_{r}[k]\right\|^{2}\right] .
\end{aligned}
$$

Let $\boldsymbol{i}_{r}$ denote the indicator function of interference in the samples $\boldsymbol{z}_{r}$ on the $r$ th receiver:

$$
\begin{gathered}
\boldsymbol{i}_{r}=\left[\begin{array}{c}
i[0] \\
\vdots \\
i[n] \\
\vdots \\
i[n=N]
\end{array}\right] \\
i_{r}[n]= \begin{cases}1 & \text { if interference is present in time sample } n \\
0 & \text { otherwise }\end{cases}
\end{gathered}
$$




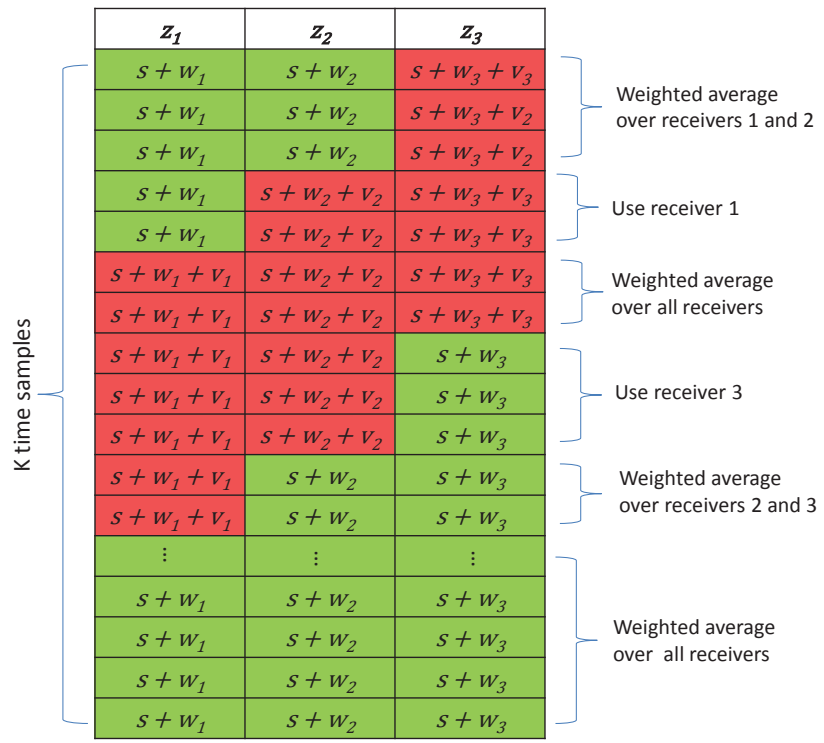

(a)

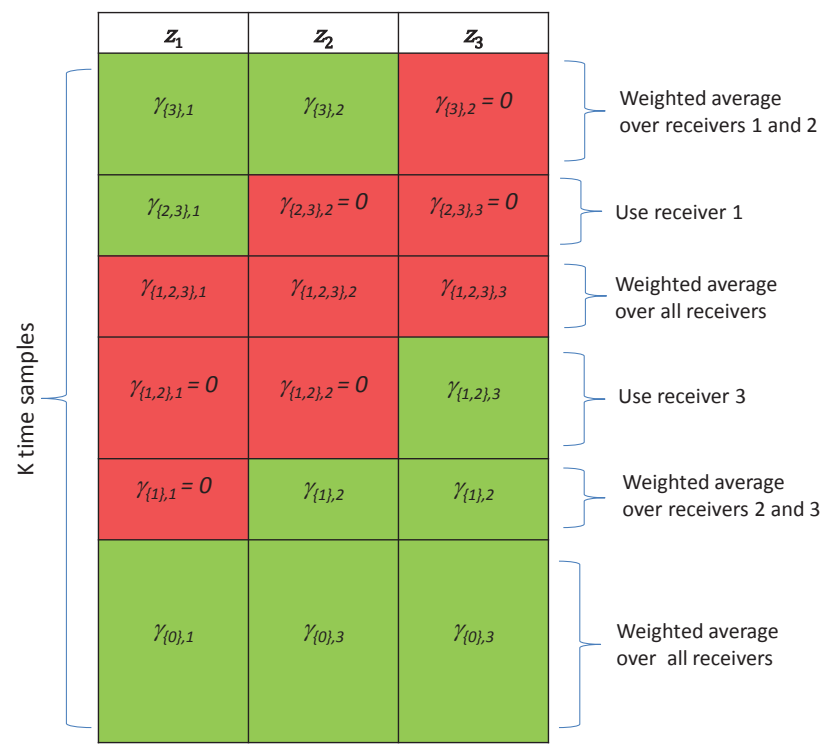

(b)

Figure 15: Weighted combining for 3 receivers suffering non-orthogonal time domain interference.

Time domain received signal with additive noise and non-orthogonal time domain interference (panel a). The SDR weighting groups (panel b) where $\gamma_{\left\{i_{1}, \ldots, i_{I}\right\}, r}$ indicates the weight for receiver $r$ when receivers $\left\{i_{1}, \ldots, i_{I}\right\}$ suffer interference concurrently. Red indicates samples suffering interference; green indicates samples suffering from only noise. 
Concatenate the interference indicator vectors $\boldsymbol{i}_{r}$ into a matrix $\boldsymbol{I}_{n, r}$. Similarly, concatenate the received signals into a matrix $\boldsymbol{Z}_{n, r}$. The signal reconstruction operation on the $R$ receivers is then defined by

$$
\boldsymbol{z}^{\mathrm{SDR}}= \begin{cases}\frac{\sum_{r} \boldsymbol{Z}_{n, r} \circ \bar{\alpha}_{r}\left[\mathbf{1}-\boldsymbol{I}_{n, r}\right]}{\sum_{r} \bar{\alpha}_{r}\left[\mathbf{1}-\boldsymbol{I}_{n, r}\right]} & \text { where } \sum_{r} \boldsymbol{I}_{n, r}<R \quad \text { (a) } \\ \frac{\sum_{r} \boldsymbol{Z}_{n, r} \circ \alpha_{r} \boldsymbol{I}_{n, r}}{\sum_{r} \alpha_{r} \boldsymbol{I}_{n, r}} & \text { where } \sum_{r} \boldsymbol{I}_{n, r}=R \quad \text { (b) }\end{cases}
$$

where 1 is a matrix of all ones, o denotes the Hadamard (element-wise) matrix product and the division operation is also an element-wise division. Noncontemporaneous interference is excised from the reconstructed signal by the operation in Eq. (61-a) and averaging is performed across all portions of the signals where the interference occurs concurrently on all receivers as given by Eq. (61b). The weights in Eq. (61-a) are based on only the noise on the receivers while the weighting in Eq. (61-b) accounts for the different noise and interference levels among all the receivers. The weighting in Eq. (61) can be partitioned into groups according the time samples suffering interference on each receiver as shown for the example in Fig. 15 where $\gamma_{\left\{i_{1}, \ldots, i_{I}\right\}, r}$ is the weight for receiver $r$ when receivers $\left\{i_{1}, \ldots, i_{I}\right\}$ suffer interference concurrently.

\subsubsection{Re-equalization, Data Detection and Decoding}

Taking the Fourier transform of the reconstructed time domain signal $z^{\mathrm{SDR}}$ produces the reconstructed signal in the frequency domain $\mathbf{z}^{\mathrm{SDR}}$. After running the equalizer (from section 2.4.2) on $\mathbf{z}^{\mathrm{SDR}}$, the symbols are extracted. Finally, the LDPC decoder is run on the detected symbols producing the decoded message. 


\subsection{Sources of Error}

Equalization plays a critical role in the Spatial Diversity Reconstruction algorithm since the reconstruction process operates on equalized waveforms. In single channel communication systems, the equalization errors manifest as differences in the amplitude and/or phase of the equalized waveform from the transmitted waveform. Since the SDR algorithm combines time domain waveforms from different receivers, it is potentially prone to an additional source of error resulting from amplitude and/or phase discontinuities in the reconstructed waveform, which arise from mismatches in amplitude and/or phase in the equalized waveforms among the various receivers. These discontinuities can occur at the transitions in the time domain signal from regions of interference and noise to regions of interference only because the SDR processing blanks time domain portions of the signal suffering interference and averages over the portions of the waveform suffering from only noise. The re-equalization of the reconstructed waveform prior to symbol detection ameliorates some of the impact from discontinuities.

Consider Figs. 16 and 17 as examples of how amplitude and phase discontinuities can arise. These figures show the SDR algorithm operating on the time domain waveforms of a single carrier frequency (e.g. a sinusoid) received on two different channels subject to either amplitude (Fig. 16) or phase (Fig. 17) errors. The received waveform $\boldsymbol{z}_{r}$ is modeled as:

$$
\boldsymbol{z}_{r}=\left(1+A_{\mathrm{err}_{r}}\right) \boldsymbol{s} e^{j \phi_{\mathrm{err}_{r}}}
$$

where $A_{\operatorname{err}_{r}}$ is the amplitude error and $\phi_{\operatorname{err}_{r}}$ is the phase error on receiver $r$. In panel (a) of Fig. 16 the real part of the received signal $z_{1}$ (in red) suffers an amplitude

error $A_{\mathrm{err}_{1}}=.5$ and no phase error $\left(\phi_{\operatorname{err}_{r}}=0\right)$. Similarly, in panel (b) the real part of the received signal $z_{1}$ (in blue) suffers only an amplitude error $A_{\mathrm{err}_{2}}=-0.5$. The real part of transmitted signal $s$ is shown in grey for comparison and the 
interference $\boldsymbol{i}_{r}$ is indicated by the black line in both panels (a) and (b). Processing these receptions with the SDR algorithm results in the waveform $z^{\mathrm{SDR}}$, the real part of which is shown in panel (c). The red portion is taken from only $\boldsymbol{z}_{1}$, the blue portion comes from only $\boldsymbol{z}_{2}$ and the green portion results from averaging $\boldsymbol{z}_{1}$ and $\boldsymbol{z}_{2}$. The discontinuities are readily apparent. Panel (d) shows the absolute error between the transmitted and reconstructed waveform $\left|s-z^{\mathrm{SDR}}\right|$. Fig. 17 shows the discontinuities induced by a $20^{\circ}$ phase mismatch, $\boldsymbol{z}_{r}=\boldsymbol{s} e^{j \phi_{\operatorname{err} r}}, \phi_{\operatorname{err}_{1,2}}=\left\{ \pm 10^{\circ}\right\}$, respectively. Looking beyond a single carrier frequency, Fig. 18 shows the results of a $20^{\circ}$ phase mismatch applied to the OFDM waveform used in the simulations and experiments discussed in the next chapter.

In order to gain understanding of the effects mismatches in the equalization process induce in overall performance, the SDR algorithm was applied to an OFDM waveform in which errors in amplitude $A_{\text {err }}$ and phase $\phi_{\text {err }}$ were artificially inserted on two receivers as follows:

$$
\begin{aligned}
& z_{1}=\left(1+A_{\mathrm{err}}\right) \boldsymbol{s} e^{-j \phi_{\mathrm{err} / 2}} \\
& \boldsymbol{z}_{2}=\left(1-A_{\mathrm{err}}\right) \boldsymbol{s} e^{+j \phi_{\mathrm{err} / 2}}
\end{aligned}
$$

The amplitude error was varied between 0 and 0.9 and the phase mismatch was varied between 0 and 180 degrees. The length of the interference was $N_{I}=342$ samples, approximately one third of the received signal, and was separated by 171 samples on the two receivers. The SDR algorithm was applied to all combinations of received signals suffering from the range of amplitude and phase mismatches. Figs. 19 and 20 show examples of the received constellation resulting from different mismatch conditions. The black dot indicates the transmitted signal constellation and the green dots indicate correctly detected received symbols. Red x's indicate incorrectly detected symbols. In Fig. 19, there is a $20^{\circ}$ phase mismatch but no amplitude error, and in Fig. 20, there is an amplitude error of 0.4 and phase offset 
A

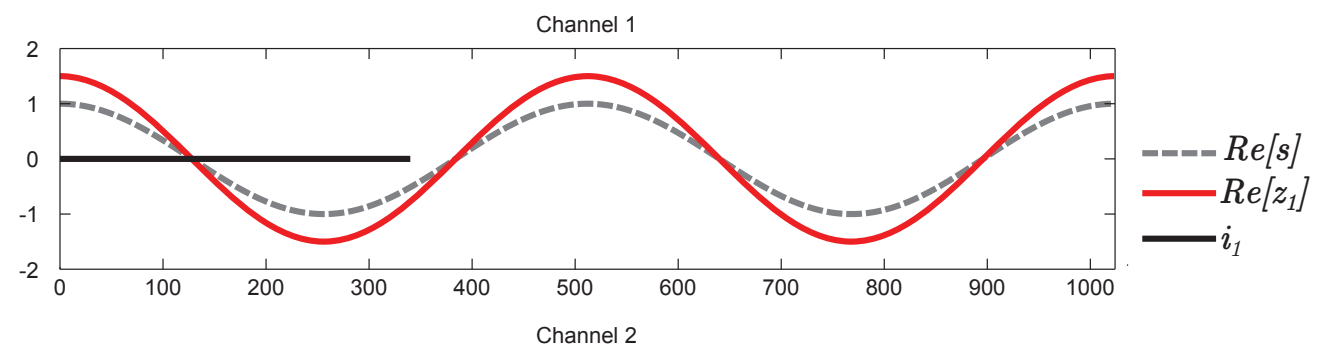

B

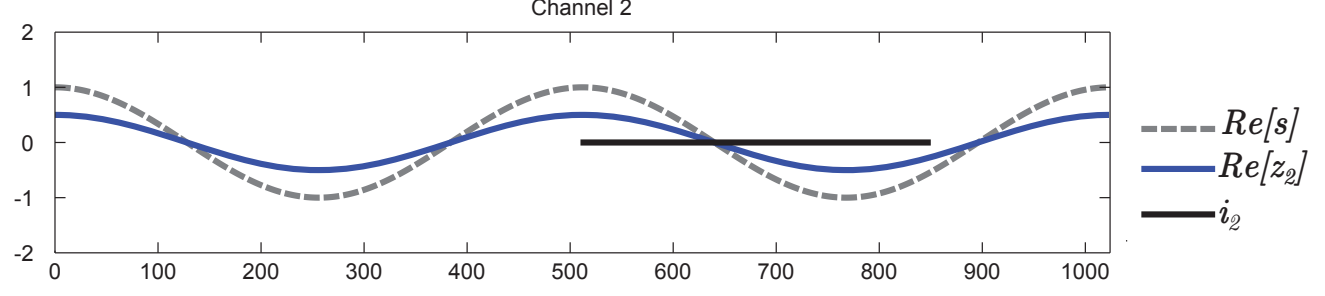

C

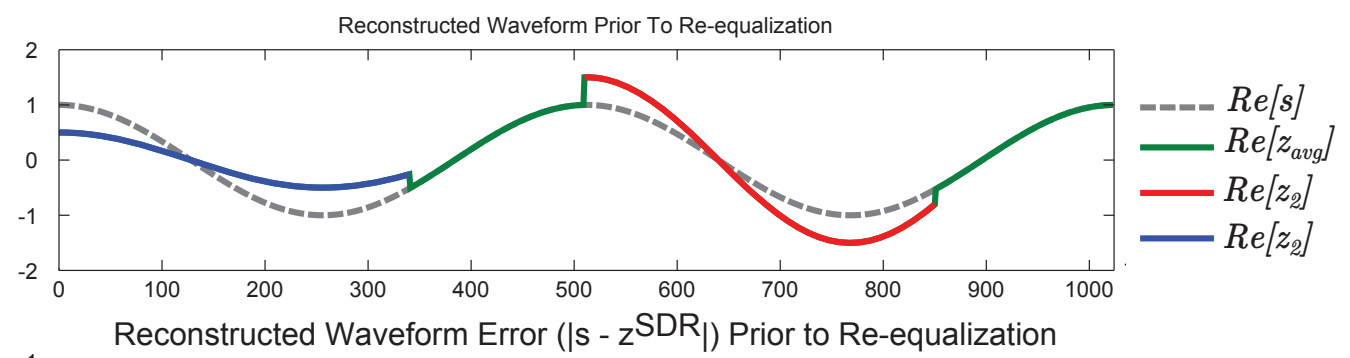

D

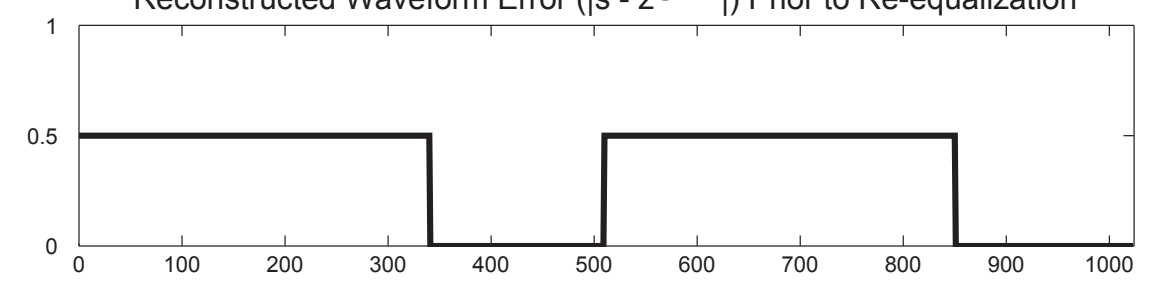

Figure 16: SDR processing a single carrier with 50\% amplitude mismatch. 
A

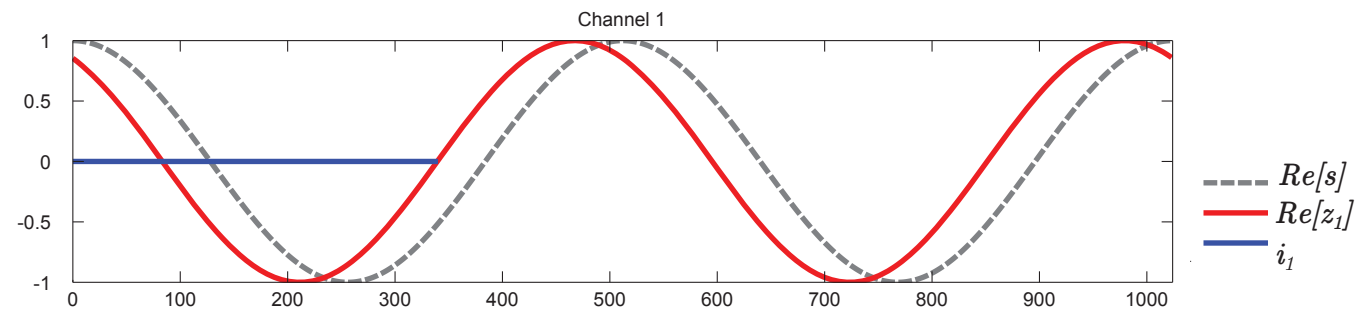

B

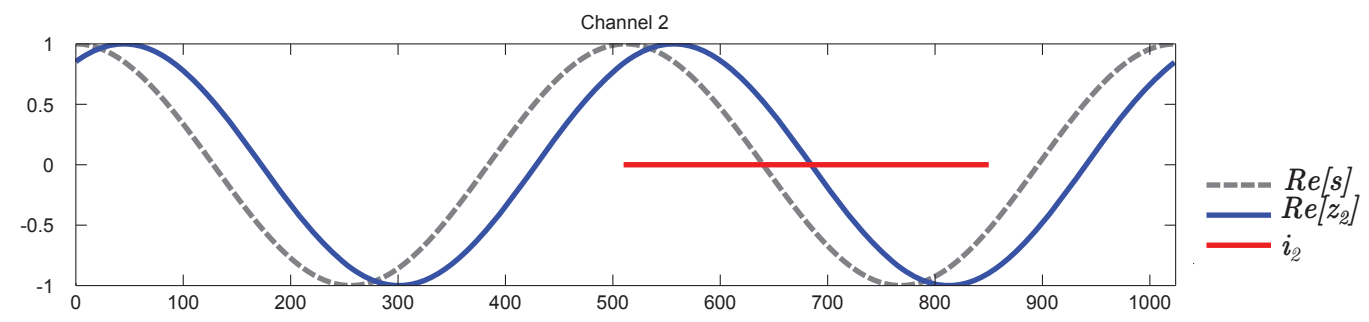

C
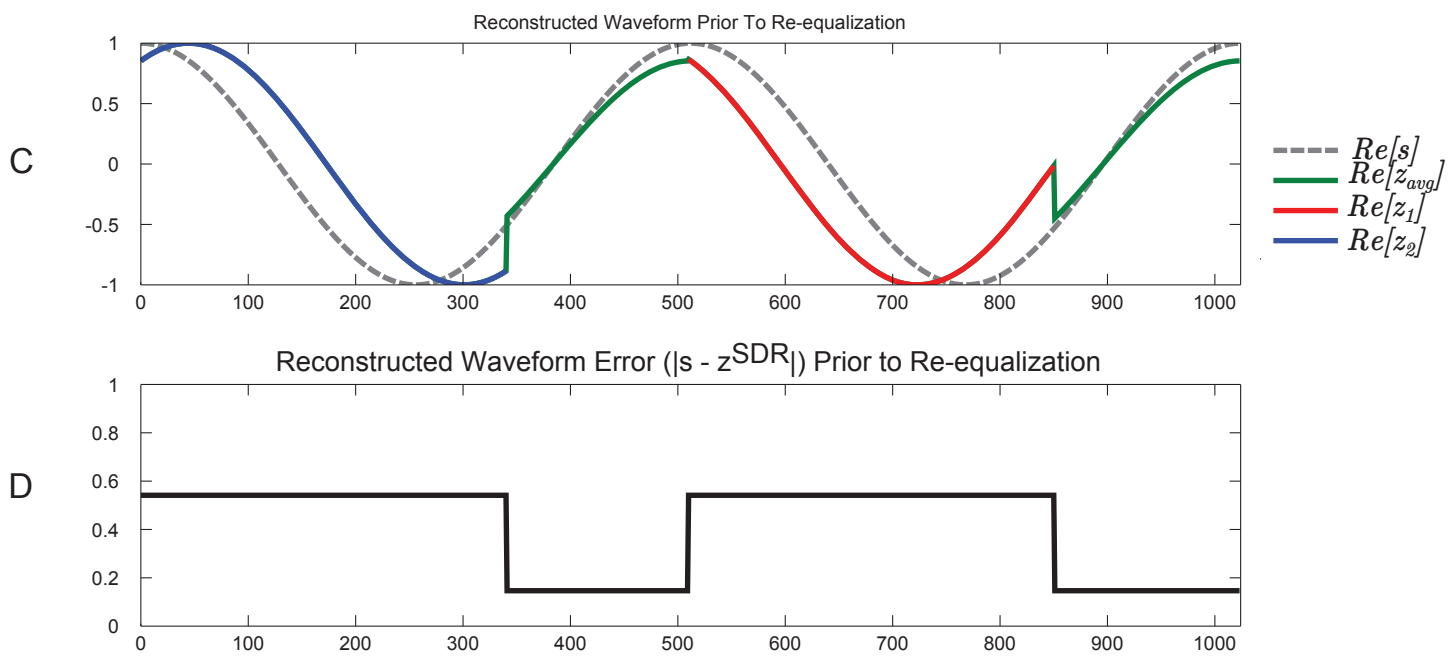

Figure 17: SDR processing a single carrier with $20^{\circ}$ phase mismatch. 
of $+100^{\circ}$ on receiver 1 and an amplitude error of -0.4 and phase offset of $-100^{\circ}$ on receiver 2. These results are typical for those observed for other combinations of amplitude and phase equalization mismatches indicating that the time domain equalization discontinuities manifest as additional noise in the OFDM symbol domain. Fig. 21 shows the number of symbol errors as a function of the inserted mismatches. For the modulation scheme (BPSK) and postulated interference used, Fig. 21 indicates that phase mismatches are more detrimental to performance, but that relatively large phase mismatches are tolerable. Phase mismatches of less than $90^{\circ}$ did not produce errors.

\subsection{Mitigation of Partial Band Partial Block Interference through Co- herent Cancellation}

The work in [17] developed an iterative single receiver parameterized interference cancellation (PIC) algorithm to mitigate partial band interference of known time duration, $T_{I}$, and effective interference bandwidth, $B_{I}$. Since the interference is time and band limited ${ }^{1}$, it can be represented by a Fourier series. The algorithm employs an iterative approach to estimate the $\mathcal{N}_{I}=\left\lceil B_{I} T_{I}\right\rceil$ complex coefficients as well as the time delay (with respect to the start of the OFDM block) of the interference. On each iteration, the estimates of the interference and detected symbols from the previous iteration are used to construct a GLRT to determine if interference is still present. If residual interference is detected, its model parameters are calculated with a maximum-likelihood approach. The modeled interference is then coherently subtracted from the desired signal, the channel is equalized, and symbol estimation is again attempted. This is repeated until the parity checks are

\footnotetext{
${ }^{1}$ The signal $s(t) \leftrightarrow S(f)$ is time and band limited if

$$
|s(t)|<\sigma_{t}^{2} \quad \text { for } t<t_{0} \text { and } t>t_{1}
$$$$
|S(f)|<\sigma_{f}^{2} \quad \text { for } f<f_{0} \text { and } f>f_{1} \text {. }
$$ 

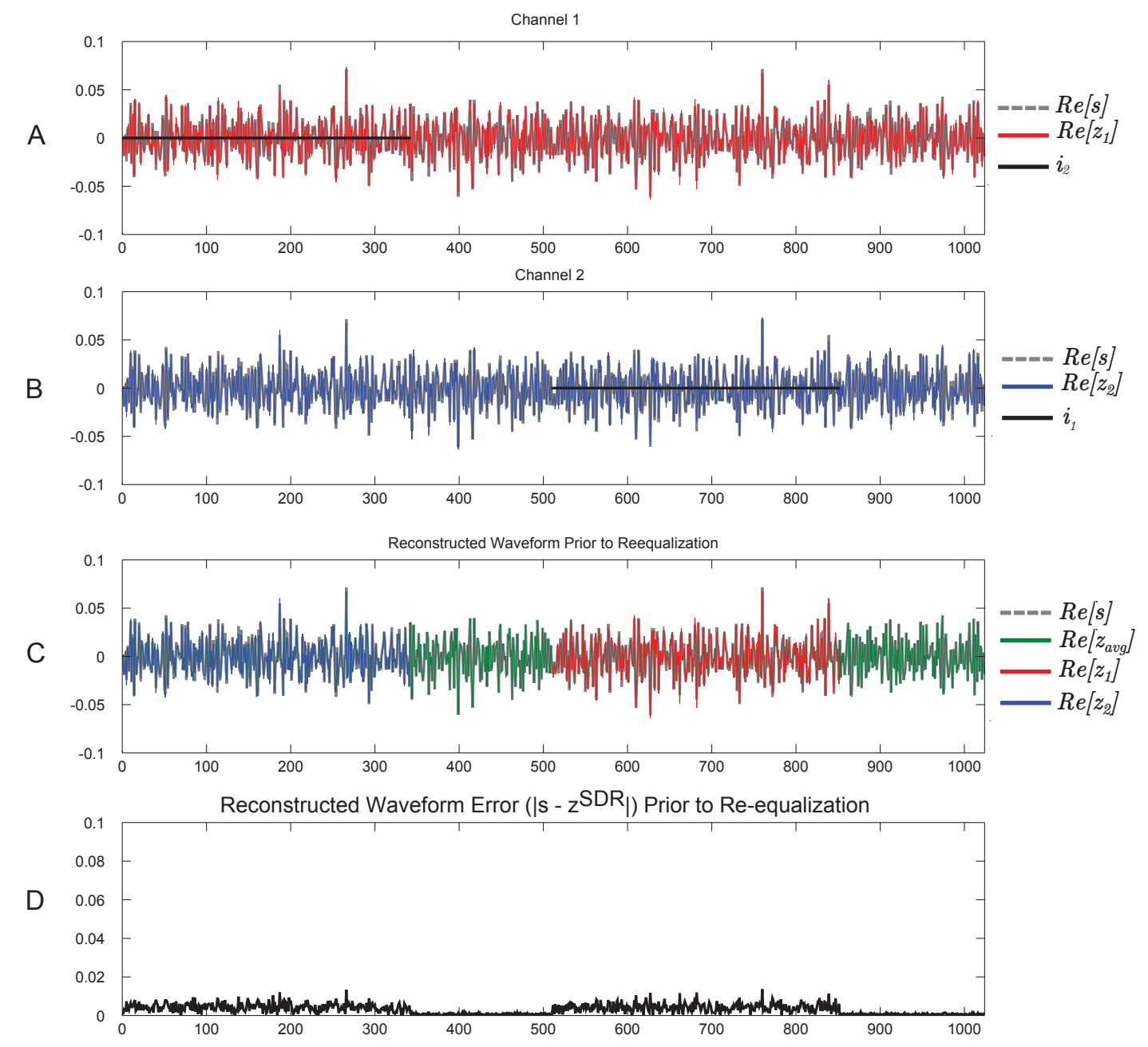

Figure 18: SDR processing an OFDM waveform with $20^{\circ}$ phase mismatch. 


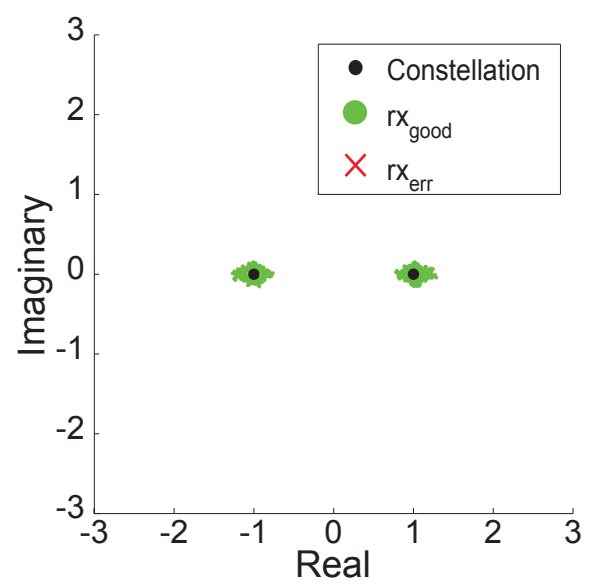

Figure 19: Signal constellation resulting from SDR processing with phase mismatch.

The received signal constellation resulting from a phase offset of $+10^{\circ}$ on receiver 1 and $-10^{\circ}$ degrees on receiver 2 .

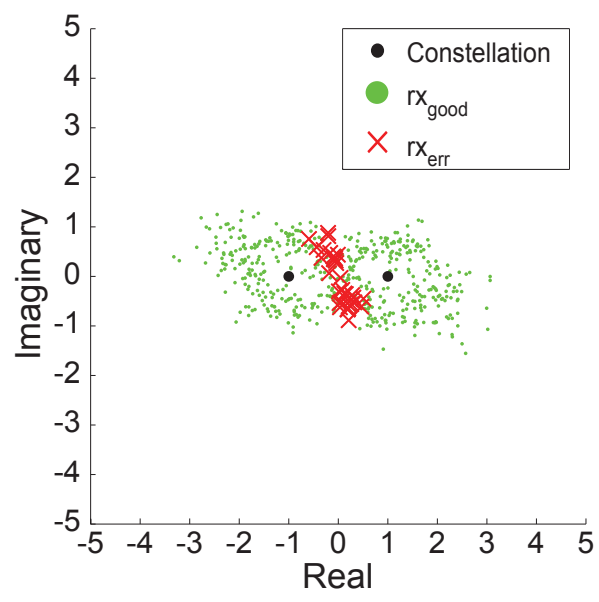

Figure 20: Signal constellation resulting from SDR processing with amplitude and phase mismatch..

The received signal constellation resulting from an amplitude error of 0.4 and phase offset of $+100^{\circ}$ on receiver 1 and an amplitude error of -0.4 and phase offset of $-100^{\circ}$ on receiver 2. Errors were made on 34 of the 512 detected symbols resulting in a BER of $6.6 \%$. 


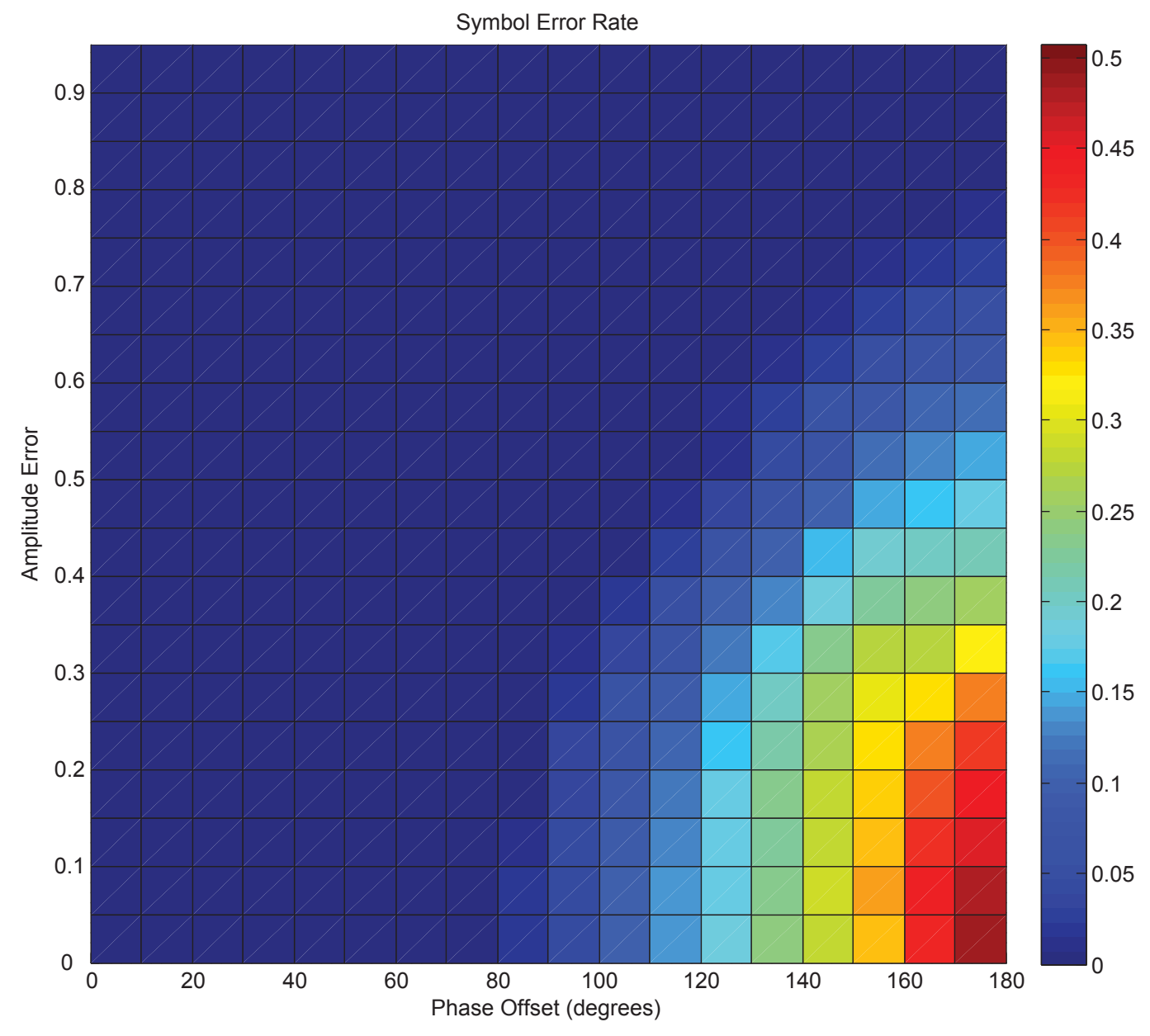

Figure 21: SDR Processing with various mismatches. 
satisfied or some maximum preset iteration limit is reached.

\subsubsection{Interference Parameterization}

The interference parameterization from [17] is repeated below to provide context and completeness. Let $\tilde{I}(t)$ denote the bandpass waveform of the interference having center frequency $f_{I_{c}}$, bandwidth $B_{I}$ and time duration $T_{I}$, and let $I(t)$ denote the baseband waveform. Since $I(t)$ is time limited, it adopts a Fourier series representation as

$$
I(t)=\sum_{l=-\infty}^{\infty} c_{l} e^{j 2 \pi \frac{l}{T_{I}} t}, \quad t \in\left[0, T_{I}\right]
$$

where $c_{l}$ is the coefficient on the basis $e^{j 2 \pi \frac{l}{T_{I}} t}$. Since $I(t)$ is bandwidth limited to $\left[\frac{-B_{I}}{2} \frac{B_{I}}{2}\right)$, the coefficient $c_{l}$ is approximately zero for $l<\frac{-\mathcal{N}_{I}}{2}$ or $l>\frac{\mathcal{N}_{I}}{2}$, where $\mathcal{N}_{I}=\left\lceil B_{I} T_{I}\right\rceil$ and $\mathcal{N}_{I}$ is assumed to be even without loss of generality. Therefore, Eq. (65) can be rewritten as

$$
I(t) \approx \sum_{l=-\frac{\mathcal{N}_{I}}{2}}^{\frac{\mathcal{N}_{I}}{2}-1} c_{l} e^{j 2 \pi \frac{l}{T_{I}} t}, \quad t \in\left[0, T_{I}\right]
$$

The corresponding bandpass signal is

$$
\tilde{I}(t)=2 \operatorname{Re}\left\{\sum_{l=-\frac{\mathcal{N}_{I}}{2}}^{\frac{\mathcal{N}_{I}}{2}-1} c_{l} e^{j 2 \pi \bar{f}_{l} t}\right\}, \quad t \in\left[0, T_{I}\right]
$$

where $\bar{f}_{l}=f_{I c}+\frac{l}{T_{I}}$. The Fourier transform of $\tilde{I}(t)$ in the frequency band $\mathcal{B}_{I}:=$ $\left[f_{I c}-\frac{B_{I}}{2}, f_{I c}+\frac{B_{I}}{2}\right]$ can be expressed as

$$
\tilde{\mathcal{I}}(f)=\sum_{l=-\frac{\mathcal{N}_{I}}{2}}^{\frac{\mathcal{N}_{I}}{2}-1} c_{l} \frac{\sin \left(\pi\left(f-\bar{f}_{l}\right) T_{I}\right)}{\pi\left(f-\bar{f}_{l}\right)} e^{-j \pi\left(f-\bar{f}_{l}\right) T_{I}}, \quad \forall f \in \mathcal{B}_{I}
$$

Note that the interference overlaps the received OFDM signal with an unknown delay. Define $\tau_{I}^{\prime}$ as the delay of the interference relative to the starting point of the OFDM block in which it resides. After the pre-processing of the OFDM receiver, 
the interference component at the $m$ th subcarrier is

$$
\nu[m]=\frac{1}{T} \int_{0}^{T+T_{g}} \tilde{I}\left(\frac{t-\tau_{I}^{\prime}}{1+\hat{a}}\right) e^{-j 2 \pi\left(f_{m}+\hat{\epsilon}\right) t} d t .
$$

Following the derivation to Eq. (35) in chapter 1, we can formulate $\nu[m]$ as

$$
\nu[m]=e^{-j 2 \pi \frac{m}{T} T_{I}} \sum_{l=-\frac{\mathcal{N}_{I}}{2}}^{\frac{\mathcal{N}_{I}}{2}-1} \rho_{m, l} u_{l}
$$

where

$$
\begin{aligned}
u_{l} & =\frac{(1+\hat{a}) T_{I}}{T} e^{-j 2 \pi\left(f_{c}+\hat{\epsilon}\right) T_{I}} c_{l}, \quad \tau_{I}=\frac{\tau_{I}^{\prime}}{1+\hat{a}} \\
\rho_{m, l} & =\frac{\sin \left(\pi\left((1+\hat{a})\left(f_{m}+\hat{\epsilon}\right)-\bar{f}_{l}\right) T_{I}\right)}{\pi\left((1+\hat{a})\left(f_{m}+\hat{\epsilon}\right)-\bar{f}_{l}\right) T_{I}} e^{-j \pi\left((1+\hat{a})\left(f_{m}+\hat{\epsilon}\right)-\bar{f}_{l}\right) T_{I}}
\end{aligned}
$$

Stacking interference components at all subcarriers into a vector $\nu$ yields

$$
\nu=\Lambda\left(\tau_{I}\right) \boldsymbol{\Gamma}_{I} \mathbf{u}
$$

where $\boldsymbol{\Lambda}\left(\tau_{I}\right)$ is a $K \times K$ diagonal matrix, $\boldsymbol{\Gamma}_{I}$ is a $K \times \mathcal{N}_{I}$ matrix, and $\mathbf{u}$ is an $\mathcal{N}_{I} \times 1$ vector

$$
\begin{aligned}
{\left[\boldsymbol{\Lambda}\left(\tau_{I}\right)\right]_{m, m} } & =e^{-j 2 \pi \frac{m}{T} \tau_{I}}, \quad\left[\boldsymbol{\Gamma}_{I}\right]_{m, l}=\rho_{m, l} \\
\mathbf{u} & =\left[u_{-\frac{\mathcal{N}_{I}}{2}}, \cdots, u_{\frac{\mathcal{N}_{I}}{2}-1}\right]^{T} .
\end{aligned}
$$

The received signal in the presence of interference is then formulated as

$$
\mathbf{z}=\mathbf{H} \mathbf{s}+\boldsymbol{\Lambda}\left(\tau_{I}\right) \boldsymbol{\Gamma}_{I} \mathbf{u}+\mathbf{w}
$$

\subsubsection{Interference Detection and Estimation}

Assume that both channel and symbol estimates $(\hat{\mathbf{H}}$ and $\hat{\mathbf{s}})$ are available. Within the interference band $\mathcal{B}_{I}$, there are $\mathcal{M}_{I}=\left\lceil B_{I} T\right\rceil$ subcarriers contaminated. Denote the set of subcarriers within the interference band as $\left\{i_{1}, \ldots, i_{\mathcal{M}_{I}}\right\}$. Define a selector matrix $\Theta$ of size $\mathcal{M}_{I} \times K$ with unity entry at the $\left(m, i_{m}\right)$ th position 
$\left(m=1, \ldots, \mathcal{M}_{I}\right)$ and zeros elsewhere. The selector matrix $\boldsymbol{\Theta}$ is determined based on the prior knowledge of the interference frequency band.

The relevant measurements within the interference frequency band are contained in

$$
\overline{\mathbf{z}}=\boldsymbol{\Theta}(\mathbf{z}-\hat{\mathbf{H}} \hat{\mathbf{s}})=\mathbf{B}\left(\tau_{I}\right) \mathbf{u}+\overline{\mathbf{w}}
$$

where

$$
\mathbf{B}\left(\tau_{I}\right):=\boldsymbol{\Theta} \Lambda\left(\tau_{I}\right) \boldsymbol{\Gamma}_{I}, \quad \overline{\mathbf{w}}:=\boldsymbol{\Theta} \mathbf{w}+\boldsymbol{\Theta}(\mathbf{H} \mathbf{s}-\hat{\mathbf{H}} \hat{\mathbf{s}})
$$

Here, $\overline{\mathbf{w}}$ denotes the equivalent additive noise within the frequency band, which consists of the ambient noise and the residual noise due to imperfect channel and information symbol estimates.

Assume that the noise samples are independent and follow a complex Gaussian distribution $\mathcal{C N}\left(0, \sigma_{B_{I}}^{2} \mathbf{I}_{\mathcal{M}_{I}}\right)$, where $\sigma_{B_{I}}^{2}$ denotes the noise variance. The likelihood function of the measurement component $\overline{\mathbf{z}}$ in the presence of interference is

$$
f\left(\overline{\mathbf{z}} \mid \tau_{I}, \mathbf{u}\right) \propto \exp \left[-\frac{1}{\sigma_{B_{I}}^{2}}\left\|\overline{\mathbf{z}}-\mathbf{B}\left(\tau_{I}\right) \mathbf{u}\right\|^{2}\right] .
$$

Let $\mathcal{H}_{0}$ and $\mathcal{H}_{1}$ denote the absence and presence of interference, respectively. To detect the presence of interference in one particular OFDM block, define the GLRT statistic

$$
\begin{aligned}
L(\overline{\mathbf{z}}) & =\max _{\left\{\tau_{I}, \mathbf{u}\right\}} \log \frac{f\left(\overline{\mathbf{z}} \mid \tau_{I}, \mathbf{u}, \mathcal{H}_{1}\right)}{f\left(\overline{\mathbf{z}} \mid \mathcal{H}_{0}\right)} \\
& =\max _{\left\{\tau_{I}, \mathbf{u}\right\}} \log \frac{\exp \left[-\frac{\left\|\overline{\mathbf{z}}-\mathbf{B}\left(\tau_{I}\right) \mathbf{u}\right\|^{2}}{\sigma_{B_{I}}^{2}}\right]}{\exp \left[-\frac{\|\overline{\mathbf{z}}\|^{2}}{\sigma_{B_{I}}^{2}}\right]} \\
& =\max _{\left\{\tau_{I}, \mathbf{u}\right\}} \frac{1}{\sigma_{B_{I}}^{2}}\left[\overline{\mathbf{z}}^{H} \mathbf{B}\left(\tau_{I}\right) \mathbf{u}+\mathbf{u}^{H} \mathbf{B}^{H}\left(\tau_{I}\right) \overline{\mathbf{z}}-\mathbf{u}^{H} \mathbf{B}^{H}\left(\tau_{I}\right) \mathbf{B}\left(\tau_{I}\right) \mathbf{u}\right] \lessgtr \Gamma_{t h}(77)
\end{aligned}
$$

where $\Gamma_{t h}$ is a predetermined threshold.

Define an objective function to be maximized over $\left\{\tau_{I}, \mathbf{u}\right\}$ 


$$
J \triangleq \overline{\mathbf{z}}^{H} \mathbf{B}\left(\tau_{I}\right) \mathbf{u}+\mathbf{u}^{H} \mathbf{B}^{H}\left(\tau_{I}\right) \overline{\mathbf{z}}-\mathbf{u}^{H} \mathbf{B}^{H}\left(\tau_{I}\right) \mathbf{B}\left(\tau_{I}\right) \mathbf{u}
$$

Typically for an optimization problem, the maximization must be carried out jointly over the parameters, in this case $\mathbf{u}$ and $\tau_{I}$. However, successful results were achieved in [17] by finding the optimal estimate of Fourier coefficients $\mathbf{u}$ and then maximizing over the delay, $\tau_{I}$. This was accomplished by setting $\nabla J_{\mathbf{u}}$ to zero to yield the optimal estimate of $\mathbf{u}$ as

$$
\hat{\mathbf{u}}=\left[\mathbf{B}\left(\tau_{I}\right)^{H} \mathbf{B}\left(\tau_{I}\right)\right]^{-1} \mathbf{B}\left(\tau_{I}\right)^{H} \mathbf{z}
$$

substituting $\hat{\mathbf{u}}$ into Eq. (78) to obtain

$$
\hat{\tau}_{I}=\arg \max _{\tau_{I}} \overline{\mathbf{z}}^{H} \mathbf{B}\left(\tau_{I}\right)\left[\mathbf{B}\left(\tau_{I}\right)^{H} \mathbf{B}\left(\tau_{I}\right)\right]^{-1} \mathbf{B}\left(\tau_{I}\right)^{H} \overline{\mathbf{z}}
$$

and solving Eq. (80) by one dimensional grid search over the delay. Based on the estimated parameters $\left\{\hat{\mathbf{u}}, \hat{\tau}_{I}\right\}$, the test statistic is evaluated as

$$
L(\overline{\mathbf{z}})=\frac{1}{\hat{\sigma}_{\mathcal{B}_{I}}^{2}} \hat{\mathbf{u}}^{H} \mathbf{B}^{H}\left(\hat{\tau}_{I}\right) \mathbf{B}\left(\hat{\tau}_{I}\right) \hat{\mathbf{u}} \gtrless \Gamma_{t h} .
$$

The test statistic $\mathrm{E}(\overline{\mathbf{z}})$ is the ratio of the energy of the estimated interference to the energy of the equivalent noise. The test threshold $\Gamma_{t h}$ is determined based on the predetermined probability of false alarm $P_{F A}$ or the probability of detection $P_{D}$. In our experience with the algorithm, we noted that there was no loss in performance

in applying the cancellation algorithm in the absence of interference. The cost of false alarms is negligible. However, not applying the algorithm when interference is present significantly degrades performances. Consequently, it is best to set the threshold to maximize probability of detection.

\subsubsection{Channel Estimation, Equalization and LDPC Decoding}

If the presence of interference is declared from the GLRT detector, the desired OFDM component can be obtained by subtracting the estimated interference from 
the received signal

$$
\breve{\mathbf{z}}=\mathbf{z}-\Lambda\left(\hat{\tau}_{I}\right) \Gamma_{I} \hat{\mathbf{u}}=\mathbf{H} \mathbf{s}+\breve{\mathbf{w}}
$$

where $\breve{\mathbf{w}}$ denotes the equivalent noise which consists of the ambient noise and the residual interference

$$
\breve{\mathbf{w}}=\mathbf{w}+\left[\Lambda\left(\tau_{I}\right) \Gamma_{I} \mathbf{u}-\Lambda\left(\hat{\tau}_{I}\right) \Gamma_{I} \hat{\mathbf{u}}\right]
$$

If no interference is detected (either absence of interference or missed detection), simply set $\hat{\mathbf{u}}=0$ in Eq. (82). Based on the observation vector $\breve{\mathbf{z}}$ and the symbol vector $\hat{\mathbf{s}}$, the channel estimator in [32] is used to estimate the channel matrix H. This estimator assumes a diagonal channel matrix and is appropriate for the channel observed in the AUTEC-0514 experiment. The work in [17] used the more sophisticated channel estimator of [54] in conjunction with the MMSE estimator of [61]. The LMMSE estimate of the information symbols is fed into the LDPC decoder to obtain hard and soft decisions on the information symbols. If all the parity checks are not satisfied, another iteration of interference cancellation is performed, in which information symbols satisfying the parity check equations are used as additional pilot symbols.

\subsubsection{Noise Variance Estimation}

Due to the partial-band property of the interference, the noise variance $\sigma_{w}^{2}$ is estimated separately for the noise within and outside of the interference band $\mathcal{B}_{I}$ based on the frequency measurements at the null subcarriers. Based on the estimates of the channel matrix and the transmitted symbols, the variance of the equivalent noise outside of the interference band, which consists of the ambient noise and the residual ICI due to the banded assumption of the channel matrix, 
can be estimated as

$$
\hat{\sigma}_{\overline{\mathcal{B}}_{I}}^{2}=\mathbb{E}_{\left\{m \in \mathcal{S}_{N}, f_{m} \notin \mathcal{B}_{I}\right\}}\left[\left\|z[m]-\sum_{k=m-D}^{m+D} \hat{H}[m, k] \hat{s}[k]\right\|^{2}\right] .
$$

For the equivalent noise within the interference band, which consists of the ambient noise, the residual ICI and the residual interference, the noise variance can be estimated as

$$
Y e s \hat{\sigma}_{\mathcal{B}_{I}}^{2}=\mathbb{E}_{\left\{m \in \mathcal{S}_{N}, f_{m} \in \mathcal{B}_{I}\right\}}\left[\left\|z[m]-\sum_{k=m-D}^{m+D} \hat{H}[m, k] \hat{s}[k]-\sum_{l=-\frac{\mathcal{N}_{I}}{2}}^{\frac{\mathcal{N}_{I}}{2}} \Lambda\left(\hat{\tau}_{I}\right)[m, m] \Gamma_{I}[m, l] \hat{u}[l]\right\|^{2}\right] .
$$

Since the implementation of the equalizer assumed a diagonal channel, $D$ is taken as zero in Eqs. (84) and (85). The estimated variance is then used for interference detection and information symbol estimation.

\subsection{Comparison of Spatial Diversity Reconstruction and Parameter- ized Interference Cancellation}

The SDR algorithm and the PIC algorithm address the problem of interference in fundamentally different ways. Table 1 summarizes the differences. SDR requires multiple receivers while PIC operates on a single receiver and could be extended to multiple receivers. Some possible approaches to extending the algorithm are discussed in Chapter 5. However, it is unclear that the performance gain from adding additional receivers would be significantly better than processing the channel with the highest signal-to-interference ratio (SIR) alone. The SDR approach is to blank out portions of the time domain waveform suffering from interference, while the PIC approach estimates the interference and coherently subtracts it from the desired signal. The SDR approach thus takes advantage of the time orthogonality of the interference whereas PIC does not. In cases where the interference is

not time orthogonal, SDR improves performance by appropriately weighting the reconstructed signal based on both the SNR and SIR. Both algorithms use a priori 
knowledge to aid in frequency domain interference detection. SDR uses a priori knowledge to set the size of the time domain blanking window while in PIC $a$ priori knowledge determines the number of coefficients used to estimate the interference. In SDR, imprecise a priori knowledge leads to performance degradation because improperly sizing the blanking window results in interference contaminating the signal if the window is too small, or loss in averaging gain if the window is too large. In contrast, for PIC, if the number of coefficients to estimate is too large, the excess coefficients get little weight. Consequently, there is less loss due to imprecise prior knowledge. Furthermore for PIC, the important parameter in determining the number of coefficients to estimate is the time bandwidth product. Interfering signals with different time durations and bandwidths but a time bandwidth product less than the selected value $\mathcal{N}_{I}$ will still be estimated accurately and subtracted. In contrast, SDR requires the time duration and bandwidth to be known separately and its performance is best when applied to signals with the specified time duration and bandwidth.

While SDR requires more detailed prior knowledge than PIC, its performance is less dependent on the SIR as will be seen in the results presented in the next chapter. PIC relies on accurately estimating the channel and transmitted symbols to aid in estimating and canceling the interference. As the interference gets stronger, channel estimation and symbol detection degrade resulting in poorer estimates of the interference. At loud enough interference levels, the algorithm fails. Because SDR blanks the interference rather than attempting to cancel it, the algorithm is less susceptible to interference induced equalization errors. In contrast to PIC, there is no low SIR threshold where SDR abruptly fails. Its performance is more strongly a function of the background noise and the degree of time orthogonality of the interference than the level of the interference. 


\begin{tabular}{|c|c|}
\hline Spatial Diversity Reconstruction & Parameterized Interference Cancellation \\
\hline Requires multiple receivers. & $\begin{array}{l}\text { Single receiver algorithm which might be extended } \\
\text { to multiple receivers. However, the approach to ex- } \\
\text { tending the algorithm and the performance gained } \\
\text { from additional receivers are unclear. }\end{array}$ \\
\hline $\begin{array}{l}\text { Relies on the time orthogonality of interference for } \\
\text { blanking. }\end{array}$ & $\begin{array}{l}\text { Relies on estimating the time arrival and Fourier } \\
\text { series coefficients of the interference to coherently } \\
\text { remove it from the desired signal. }\end{array}$ \\
\hline Performance is not limited by SIR. & Performance degrades at low SIR. \\
\hline $\begin{array}{l}\text { A priori knowledge facilitates frequency domain } \\
\text { interference detection and sets the size of time do- } \\
\text { main blanking window. }\end{array}$ & $\begin{array}{l}\text { A priori knowledge sets the number of coefficients } \\
\text { used to estimate interference. }\end{array}$ \\
\hline $\begin{array}{l}\text { Imprecise prior knowledge leads to performance } \\
\text { loss. }\end{array}$ & $\begin{array}{l}\text { Prior knowledge does not need to be particularly } \\
\text { precise. A loose upper bound on the time band- } \\
\text { width product } \mathcal{N}_{I}=\left\lceil B_{I} T_{I}\right\rceil \text { is sufficient. }\end{array}$ \\
\hline $\begin{array}{l}\text { Single pass receiver: interference detection, exci- } \\
\text { sion, channel estimation and data detection are } \\
\text { separate processes. }\end{array}$ & $\begin{array}{l}\text { Interference detection, interference reconstruction } \\
\text { and cancellation, channel estimation and data de- } \\
\text { tection are coupled to form an iterative receiver. }\end{array}$ \\
\hline $\begin{array}{l}\text { Analytic expression for performance under certain } \\
\text { conditions. } 1\end{array}$ & No analytic expression for expected performance. \\
\hline $\begin{array}{l}\text { Requires operations in the time and frequency do- } \\
\text { mains to take advantage of the time orthogonality } \\
\text { of the interference. }\end{array}$ & $\begin{array}{l}\text { Operates exclusively in the frequency domain but } \\
\text { does not leverage the time orthogonality of the in- } \\
\text { terference. }\end{array}$ \\
\hline
\end{tabular}

Table 1: Comparison of the interference mitigation algorithms

Analytic expressions for the performance of SDR are available under certain conditions and the loss in performance due to mismatching the time domain interference blanking window can be determined for these cases as will be seen in the next chapter. PIC is a more complicated algorithm and analytic characterizations of performance are not available. Finally, SDR requires operations in both the time and frequency domains while PIC operates exclusively in the frequency domain.

\subsection{Summary}

Many acoustic channels suffer from interference which is neither narrowband nor impulsive. This relatively long duration partial band interference can be par-

\footnotetext{
${ }^{1}$ The analytic expression for performance an on additive white Gaussian noise channel suffering time orthogonal interference is derived in Chapter 3.
} 
ticularly detrimental to system performance. However, some parameters of the interference are usually known or can be acquired and exploited. For a single receiver, prior knowledge can facilitate interference cancellation. For multiple receivers, the prior knowledge can be used to exploit the detection and excision of portions of the received signals suffering from interference so that they can be combined more effectively. While the single receiver technique relies on accurately estimating and subtracting the interference, the multi-receiver technique exploits the time orthogonality of the interference due to the slow speed of sound propagation in water and the geographical extent of the network to combine clean portions of the received signals. 


\section{CHAPTER 3 \\ Simulation and Experimental Results}

\subsection{Introduction}

This chapter reports the performance of the Spatial Diversity Reconstruction algorithm under different channel conditions. In Section 3.2 analytic expressions are derived for the performance of SDR and Maximum Ratio Combining (MRC) on an additive white Gaussian noise channel suffering interference. The SDR and MRC expressions provide the basis for the development of an adaptive interference mitigation receiver. Section 3.3 provides simulation results for the SDR and MRC algorithms operating on a linear time invariant channel. Section 3.4 provides performance results from an experiment conducted at AUTEC in May 2014. The experiment enabled the performance of SDR to be compared with the Parameterized Interference Cancellation (PIC) algorithm on real data.

\subsection{Performance on Additive White Gaussian Noise Channel with In- terference}

The performance of the SDR and MRC algorithms over an impulse channel with additive white Gaussian noise and suffering from interference which is time orthogonal is derived in this section. The expected variance of the resulting signal is found. This variance can be used as the basis for developing an adaptive combining receiver which applies the SDR or MRC technique depending on which provides the best performance. The performance is derived under the following assumptions:

AS-1 The channels are impulsive.

AS-2 The noise is additive white Gaussian noise.

AS-3 The noise and interference are independent processes. 
AS-4 The interference is time and band limited. ${ }^{1}$

AS-5 The interference is contiguous and occurs only once within the OFDM block.

AS-6 The interference is additive white Gaussian noise.

AS-7 The interference is time orthogonal among the receivers.

As the assumptions above are relaxed, the analytic expressions of performance provided below become an upper bound of performance. The purpose of channel equalization is to remove intersymbol interference making the channel appear "impulse-like" to the follow-on processing. To the extent that the channel equalizer does not invert the channel response, that is $\mathbf{H}_{\mathrm{eq}} \mathbf{H} \neq \mathbf{I}$, and intersymbol interference remains there is a degradation in performance which can be categorized as loss due to equalization error. Noise whitening is also inherent is most communication receivers. [57] provided a noise whitening approach for underwater OFDM noting that significant gains can be realized by applying prewhitening before channel estimation. Thus, to the extent that the noise is not white, performance loss should be expected. Communication systems are also typically designed assuming that the noise is independent. As discussed previously, the OFDM carrier spacing is chosen so that carriers experience independent fading and considerable work has been done to mitigate inter-carrier interference (ICI). From one perspective, these mitigation techniques aim to restore the "noise independence" of the carriers. In applications, noise and interference are independent processes by definition. Thus, AS-1 through AS-3 are reasonable assumptions, and further, the extent to which they do not hold, performance typically degrades.

\footnotetext{
${ }^{1}$ The signal $s(t) \leftrightarrow S(f)$ is time and band limited if

$$
|s(t)|<\sigma_{t}^{2} \quad \text { for } t<t_{0} \text { and } t>t_{1}
$$$$
|S(f)|<\sigma_{f}^{2} \quad \text { for } f<f_{0} \text { and } f>f_{1} \text {. }
$$ 
Communication systems must deal with different types of interference. For many applications AS-4 and AS-5 hold exactly, while AS-6 and AS-7 are more tenuous but enable an analytic evaluation of performance under optimal conditions. Performance in non-Gaussian interference will be worse for both SDR and MRC because the weighting scheme for both algorithms is optimized for Gaussian noise. Therefore, considering all of the above, the BER equations derived in this section provide an upper bound for performance.

\subsubsection{Additive White Gaussian Noise and Time Orthogonal Interfer- ence Channel Model}

Let $\mathbf{z}_{r}$ denote the $r$ th of $R$ received baseband signal vectors in the frequency domain and let $\boldsymbol{z}_{r}$ be its time domain inverse. That is, $\mathbf{z}_{r}$ and $\boldsymbol{z}_{r}$ are $K$-point Fourier transform pairs, $\mathbf{z}_{r}[k] \leftrightarrow \boldsymbol{z}_{r}[n]$ where bold type indicates the frequency domain indexed by $k$ and italics indicates the time domain indexed by $n$. The $r$ th of $R$ time domain and frequency domain reception pairs resulting from the transmission of the signal $\mathbf{s}$ over an impulse channel suffering additive noise $\mathbf{w}$ and interference $\mathbf{v}$ is given by

$$
\begin{aligned}
\mathbf{z}_{r} & =\mathbf{s}+\mathbf{w}_{r}+\mathbf{i}_{r} \circ \mathbf{v}_{r} \\
& \downarrow \\
& \\
\boldsymbol{z}_{r} & =\boldsymbol{s}+\boldsymbol{w}_{r}+\boldsymbol{i}_{r} \circ \boldsymbol{v}_{r},
\end{aligned}
$$

where $\mathbf{i}_{r} \circ \mathbf{v}_{r}$ models the interference; $\mathbf{i}_{r}$ indicates the frequency bins suffering interference and $\mathbf{v}_{r}$ is the random variable taking on the value of the interference. The indicator function $i_{r}$ is defined as

$$
\mathrm{i}_{r}[k]= \begin{cases}1 & \text { if interference is present in bin } k \\ 0 & \text { otherwise. }\end{cases}
$$

The frequency domain noise and interference samples are complex additive white Gaussian noise distributed according to $\mathrm{w}_{r}[k] \sim \mathcal{C N}\left(0, \sigma_{w_{r}^{k}}^{2}\right)$ and $\mathrm{v}_{r}[k] \sim$ 
$\mathcal{C N}\left(0, \sigma_{v_{r}^{k}}^{2}\right)$, where the $k$ in $\sigma_{v_{r}^{k}}^{2}$ indicates the variance is in the frequency domain and the $r$ indicates the $r$ th receiver. Note that since the impulse response of the channel is the impulse function $(h[n]=\delta[n])$, there is no need to overlap and add the received waveform $\boldsymbol{z}_{r}$. The $N=K$ samples of the baseband time domain received waveform $\boldsymbol{z}_{r}$ are defined by

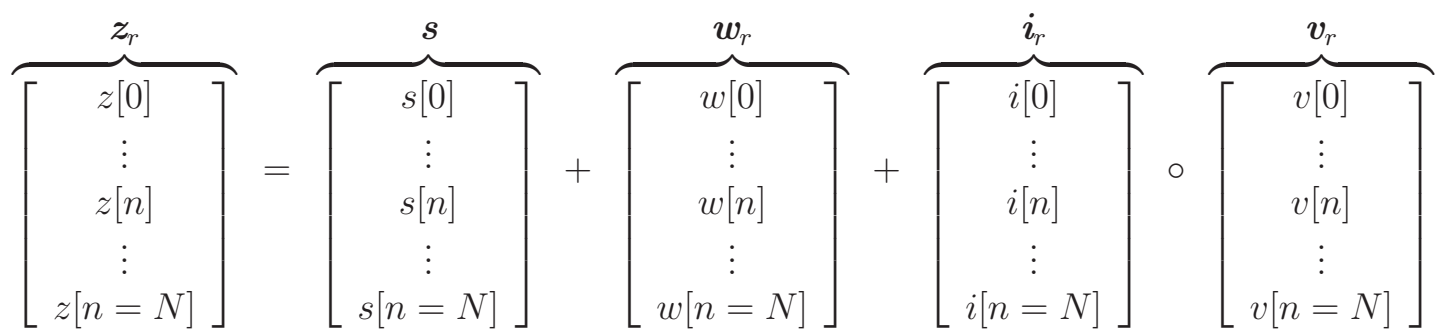

where $w_{r}[n] \sim \mathcal{C N}\left(0, \sigma_{w_{r}^{n}}^{2}\right)$ is complex additive white Gaussian noise which occurs in each time sample and $v_{r}[n] \sim \mathcal{C N}\left(0, \sigma_{v_{r}^{n}}^{2}\right)$ is the interference (also complex additive white Gaussian noise) which occurs in samples indicated by the indicator function $i_{r}$

$$
i_{r}[n]= \begin{cases}1 & \text { if interference is present in time sample } n \\ 0 & \text { otherwise }\end{cases}
$$

Following the notation above, the $n$ in $\sigma_{v_{r}^{n}}^{2}$ indicates the variance is in the time domain and the $r$ indicates the $r$ th receiver. Due to the Fourier transform, the variance of the Gaussian noise is related by the scale factor $K$. That is, $w_{r}[n] \sim$ $\mathcal{C N}\left(0, \sigma_{w_{r}^{k}}^{2} / K\right)$ and $v_{r}[n] \sim \mathcal{C N}\left(0, \sigma_{v_{r}^{k}}^{2} / K\right)$ and similarly, $\mathrm{w}_{r}[k] \sim \mathcal{C N}\left(0, K \sigma_{w_{r}^{n}}^{2}\right)$ and $\mathrm{v}_{r}[k] \sim \mathcal{C N}\left(0, K \sigma_{v_{r}^{n}}^{2}\right)$. Assume that the interference is time limited to $N_{I}$ samples and time orthogonal among the receivers. Therefore, it can be completely excised (blanked) from the reconstructed waveform. Since the interference is time orthogonal among $R$ receivers and limited to $N_{I}$ samples $R N_{I} \leq K$. Further assume the interference is band limited to $K_{I}$ frequency bins. 


\subsubsection{Spatial Diversity Reconstruction Performance}

Assuming the SDR algorithm clairvoyantly blanks the interference on the $R$ receivers, the reconstructed waveform results from the weighted average of $K-R N_{I}$ time domain samples from all $R$ receivers and the weighted average of $R N_{I}$ samples from $R-1$ receivers. Fig. 22 illustrates this for the case of three receivers. In the figure time domain samples suffering interference are indicated in red while samples suffering from only noise are shown in green. The reconstructed sample in the $n$th bin is given by:

$$
z[n]=\sum_{r=1}^{R} \alpha_{r}[n]\left(s[n]+w_{r}[n]\right)
$$

where

$$
\alpha_{r}[n]=\frac{\frac{1-i_{r}[n]}{\sigma_{w_{r}^{n}}^{2}}}{\sum_{r=1}^{R} \frac{1-i_{r}[n]}{\sigma_{w_{r}^{n}}^{2}}}
$$

is the weighting factor for the $n$th sample. The noise variance in the $n$th time sample is given by

$$
\sigma_{S D R^{n}}^{2}=\sum_{r=1}^{R} \alpha_{r}^{2}[n] \sigma_{w_{r}^{n}}^{2} .
$$

Taking the Fourier transform of the reconstructed waveform to obtain the OFDM symbol spreads the noise among all the received frequency bins. The noise variance in each frequency bin is given by:

$$
\sigma_{S D R^{k}}^{2}=\sum_{n=1}^{K} \sum_{r=1}^{R} \alpha_{r}^{2}[n] \sigma_{w_{r}^{n}}^{2} .
$$

Another form of Eq. (90) can be realized by partitioning the weighting factors according to the receivers where the averaging occurs. For $R$ receivers, there are $R+1$ weights provided some time domain samples suffer no interference. The weighting groups are 


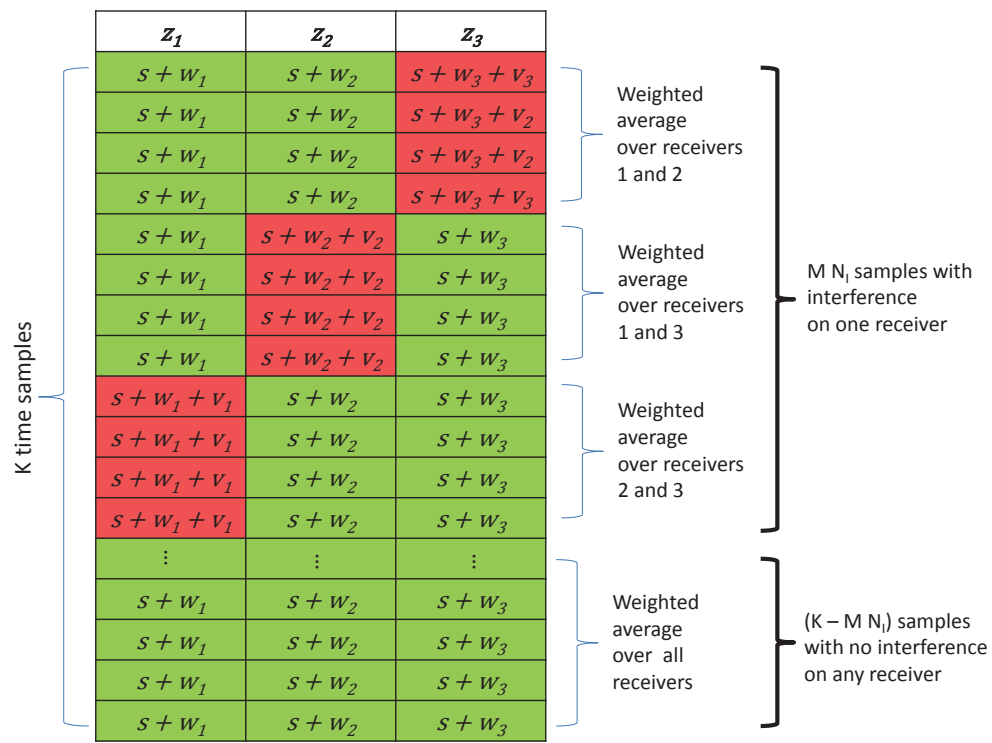

(a)

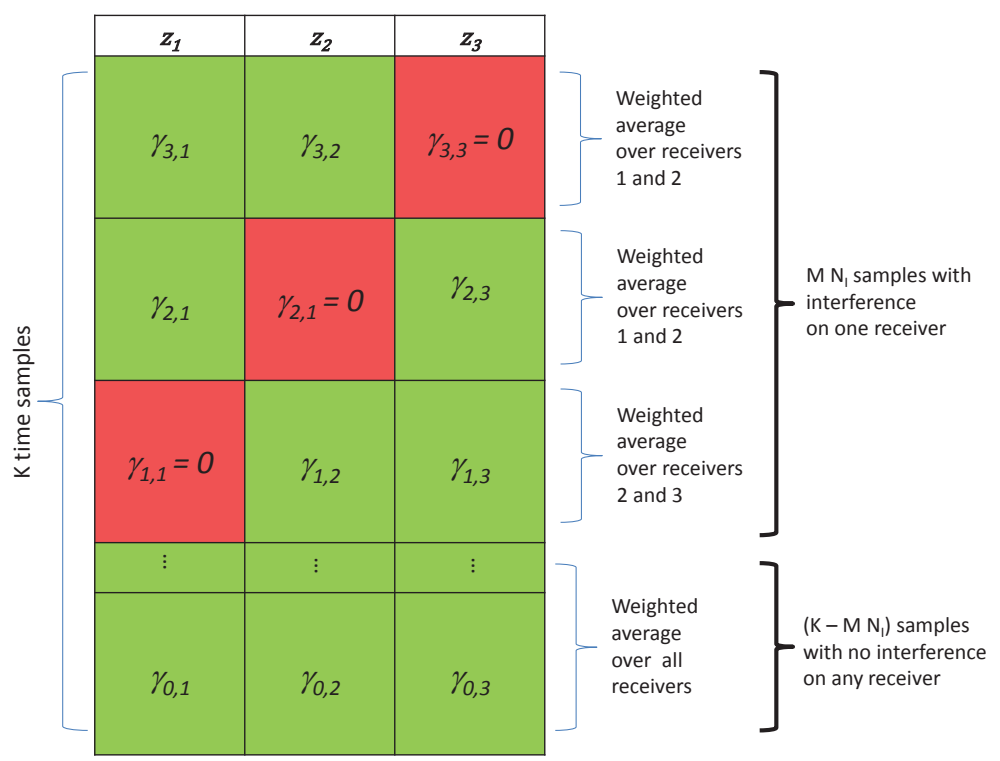

(b)

Figure 22: SDR combining strategy for three receivers with time orthogonal interference.

Time domain received signal with additive noise and time orthogonal interference (panel a). The SDR weighting groups (panel b). Red indicates samples suffering interference; green indicates samples suffering from only noise. 


$$
\begin{aligned}
& \gamma_{0, r}=\frac{\frac{1}{\sigma_{w_{r}^{n}}^{2}}}{\sum_{r=1}^{R} \frac{1}{\sigma_{w_{r}^{n}}^{2}}} \text { for } \sum_{r} i_{r}[n]=0 \\
& 0 \quad \text { for } \quad g=r, i_{g}[n]=1 \\
& \gamma_{g, r}= \begin{cases}\frac{1}{\sigma_{w_{r}^{n}}^{2}} & \text { otherwise } \\
\sum_{\{r: r \neq g\}}^{R} \frac{1}{\sigma_{w_{r}^{n}}^{2}} & \end{cases}
\end{aligned}
$$

The variance contribution from samples suffering from no interference on any receiver, (that is group $g=0$ ), is

$$
\sigma_{w_{0}^{k}}^{2}=\left(K-R N_{I}\right) \sum_{r=1}^{R} \gamma_{0, r}^{2} \sigma_{w_{r}^{n}}^{2}
$$

while the variance contribution from group $g$ is

$$
\sigma_{w_{g}^{k}}^{2}=N_{I} \sum_{r=1}^{R} \gamma_{g, r}^{2} \sigma_{w_{r}^{n}}^{2}
$$

and the total noise variance in each frequency bin is given by:

$$
\sigma_{\mathrm{SDR}^{k}}^{2}=\sigma_{w_{0}^{k}}^{2}+\sum_{g=1}^{R} \sigma_{w_{g}^{k}}^{2}
$$

Substitution of Eqs. (94) and (95) into Eq. (96) and recalling that $\sigma_{w^{n}}^{2}=\frac{\sigma_{w^{k}}^{2}}{K}$, yields an expression of the SDR frequency domain noise variance in terms of the frequency domain noise variance on each receiver,

$$
\sigma_{\mathrm{SDR}^{k}}^{2}=\frac{\left(K-R N_{I}\right)}{K} \sum_{r=1}^{R} \gamma_{0, r}^{2} \sigma_{w_{r}^{k}}^{2}+\frac{R N_{I}}{K} \sum_{g=1}^{R} \sum_{r=1}^{R} \gamma_{g, r}^{2} \sigma_{w_{r}^{k}}^{2} .
$$

The uncoded Bit Error Rate of the reconstructed received signal for BPSK is given by:

$$
\mathrm{BER}_{\mathrm{SDR}}=Q\left(\frac{\sqrt{2}}{\sigma_{\mathrm{SDR}^{k}}}\right)
$$

where $Q(x)$ is the right-tail probability (e.g. complementary cumulative distribution function) for an $\mathcal{N}(0,1)$ random variable. 


\subsubsection{Maximum Ratio Combining Performance}

In Maximum Ratio Combining, the interference is not removed from the received signal. The receptions are combined according the received noise levels. The weights $\omega_{r}$ are calculate as

$$
\omega_{r}=\frac{\frac{1}{\sigma_{w_{r}^{k}}^{2}}}{\sum_{r=1}^{R} \frac{1}{\sigma_{w_{r}^{k}}^{2}}}
$$

The noise variance in the frequency bins not suffering from interference is given by

$$
\sigma_{\mathrm{MRC}_{v=0}^{k}}^{2}=\sum_{r=1}^{R} \omega_{r}^{2}\left(\sigma_{w_{r}^{k}}^{2}\right)
$$

and the noise variance where interference is present is given by

$$
\sigma_{\mathrm{MRC}_{v \neq 0}^{k}}^{2}=\sum_{r=1}^{R} \omega_{r}^{2}\left(\sigma_{w_{r}^{k}}^{2}+\sigma_{v_{r}^{k}}^{2}\right) .
$$

The uncoded BER of the MRC received signal for BPSK is given by:

$$
\mathrm{BER}_{\mathrm{MRC}}=\frac{K-K_{I}}{K} Q\left(\frac{\sqrt{2}}{\sigma_{\mathrm{MRC}_{v=0}^{k}}^{2}}\right)+\frac{K_{I}}{K} Q\left(\frac{\sqrt{2}}{\sigma_{\mathrm{MRC}_{v \neq 0}^{2}}^{2}}\right)
$$

An "equivalent" variance for $\mathrm{MRC} \sigma_{\mathrm{MRC}_{\mathrm{eq}}^{k}}^{2}$ under interference conditions is found by inverting Eq. (102) yielding

$$
\sigma_{\mathrm{MRC}_{\mathrm{eq}}^{k}}^{2}=Q^{-1}\left(\frac{\sqrt{2}}{\mathrm{BER}_{\mathrm{MRC}}}\right)
$$

The equivalent MRC variance $\sigma_{\mathrm{MRC}_{\mathrm{eq}}^{k}}^{2}$ and the $\mathrm{SDR}$ variance $\sigma_{\mathrm{SDR}^{k}}^{2}$ can be compared to determine when to implement each combining strategy:

$$
\begin{aligned}
& \sigma_{\mathrm{MRC}_{e q}^{k}}^{2}>\sigma_{\mathrm{SDR}^{k}}^{2} \longmapsto \text { Use SDR } \\
& \sigma_{\mathrm{MRC}_{e q}^{k}}^{2}<\sigma_{\mathrm{SDR}^{k}}^{2} \longmapsto \text { Use MRC }
\end{aligned}
$$

Thus, Eqs. (96) and (103) provide the framework for developing an adaptive combining receiver which accounts for the time duration and bandwidth of the interference as well as the SIR and SNR. The development of such a receiver is 
left for future work. The implemented receiver transitioned between the MRC and blanking strategies based on the significance level of the Kolmogorov-Smirnov test.

\subsubsection{Example: Two Receivers on Additive White Gaussian Noise Channel with Interference}

For the special case of two receivers suffering time orthogonal interference on an additive white Gaussian noise channel, the variance of the noise in the $k$ th bin for the SDR algorithm from Eq. (96) is

$$
\begin{aligned}
\sigma_{S D R^{k}}^{2} & =\left(K-2 N_{I}\right) \sum_{r=1}^{2} \gamma_{0, r}^{2} \sigma_{w_{r}^{n}}^{2}+N_{I}\left(\sigma_{w_{1}^{n}}^{2}+\sigma_{w_{2}^{n}}^{2}\right) \\
& =\left(K-2 N_{I}\right)\left(\gamma_{0,1}^{2} \sigma_{w_{1}^{n}}^{2}+\gamma_{0,2}^{2} \sigma_{w_{2}^{n}}^{2}\right)+N_{I}\left(\sigma_{w_{1}^{n}}^{2}+\sigma_{w_{2}^{n}}^{2}\right) .
\end{aligned}
$$

If the noise level on the two receivers is the same, $\gamma_{0,1}=\gamma_{0,2}=\frac{1}{2}$ and the variance is

$$
\begin{aligned}
\sigma_{S D R^{k}}^{2} & =\left(K-2 N_{I}\right) \frac{\sigma_{w_{1}^{n}}^{2}+\sigma_{w_{2}^{n}}^{2}}{4}+N_{I}\left(\sigma_{w_{1}^{n}}^{2}+\sigma_{w_{2}^{n}}^{2}\right) \\
\sigma_{S D R^{k}}^{2} & =\left(\frac{K}{4}+\frac{N_{I}}{2}\right)\left(\sigma_{w_{1}^{n}}^{2}+\sigma_{w_{2}^{n}}^{2}\right) \\
\sigma_{S D R^{k}}^{2} & =\left(\frac{K}{2}+N_{I}\right) \sigma_{w^{n}}^{2}
\end{aligned}
$$

and recalling that $\sigma_{w^{n}}^{2}=\frac{\sigma_{w^{k}}^{2}}{K}$ yields

$$
\sigma_{S D R^{k}}^{2}=\left(\frac{1}{2}+\frac{N_{I}}{K}\right) \sigma_{w^{k}}^{2}
$$

resulting in a BER of

$$
\mathrm{BER}_{\mathrm{SDR}}=Q\left(\sqrt{\frac{2}{\left(\frac{1}{2}+\frac{N_{I}}{K}\right) \sigma_{w^{k}}^{2}}}\right) .
$$

For MRC, the weights are the same $\left(\omega_{1}=\omega_{2}=\frac{1}{2}\right)$ and the variance of the combined signal is

$$
\begin{aligned}
\sigma_{\mathrm{MRC}_{v=0}^{k}}^{2} & =\frac{\sigma_{w^{k}}^{2}}{2} \\
\sigma_{\mathrm{MRC}_{v \neq 0}^{k}}^{2} & =\frac{\sigma_{w^{k}}^{2}}{2}+\frac{\sigma_{v_{1}^{k}}^{2}+\sigma_{v_{2}^{k}}^{2}}{4}
\end{aligned}
$$


and the BER is given by

$$
\mathrm{BER}_{\mathrm{MRC}}=\frac{K-K_{I}}{K} Q\left(\sqrt{\frac{2}{\frac{\sigma_{w^{k}}^{2}}{2}}}\right)+\frac{K_{I}}{K} Q\left(\sqrt{\frac{2}{\frac{\sigma_{w^{k}}^{2}}{2}+\frac{\sigma_{v_{1}^{k}}^{2}+\sigma_{v_{2}^{k}}^{2}}{4}}}\right) .
$$

For the special case, when the variance of the noise $\sigma_{w^{k}}^{2}=1$, the BER performance of the two algorithms is

$$
\begin{aligned}
\mathrm{BER}_{\mathrm{SDR}} & =Q\left(\sqrt{\frac{2}{\left(\frac{1}{2}+\frac{N_{I}}{K}\right)}}\right) \\
\mathrm{BER}_{\mathrm{MRC}} & =\frac{K-K_{I}}{K} Q(2)+\frac{K_{I}}{K} Q\left(\sqrt{\frac{2}{\frac{1}{2}+\frac{\sigma_{v_{1}^{k}}^{2}+\sigma_{v_{2}^{k}}^{2}}{4}}}\right) .
\end{aligned}
$$

Plots of the performance from Eq. (108) in blue and Eq. (110) in red are shown Fig. 23, with the special case of Eqs. (111) and (112) shown in panel (a). The number of OFDM carriers is $K=1024$. The interference bandwidth varies from $3.125 \%\left(K_{I}=32\right)$ to $50 \%\left(K_{I}=512\right)$ of the band. The time duration of the interference varies similarly from $3.125 \%\left(N_{I}=32\right)$ to $50 \%\left(N_{I}=512\right)$ of the baseband symbol duration. The two receivers suffer the same SIR, ranging from -10 to 10 dB. The SDR curves demonstrate the performance gains possible from accurately blanking out the interference. For example, referring to panel (c) for two receivers operating at $6 \mathrm{~dB}$ SNR, employing a blanking window reduces the BER in all cases when the SIR is below $-2 \mathrm{~dB}$. There is some performance loss if the selected blanking window is much larger than the time duration of the interference because the averaging is done over fewer samples. For example, again referring to panel (c), for two receivers operating at $6 \mathrm{~dB}$ SNR, employing a blanking window of $N_{I_{\text {win }}}=128$ when the actual duration of the interference is $N_{I}=32$ results in an increase in the BER from $5.4 \times 10^{-5}$ to $1.8 \times 10^{-4}$. However, even with an improperly sized window, blanking still outperforms MRC at low SIR. Fig. 24 indicates the most effective combining strategy, (averaging using MRC or blanking), as a function 
of SNR, SIR, interference bandwidth and time duration for two receivers suffering equivalent noise and interference levels on an additive white Gaussian noise channel. Green indicates regions where the BER for blanking is lower than MRC, and hence, blanking should be employed. Red indicates regions where the BER for MRC is lower than blanking, and therefore, MRC should be used. The implemented SDR algorithm transitions between the two combining strategies based on the significance level of the Kolmogorov-Smirnov hypothesis test. A more adaptive implementation would transition between the MRC and blanking strategies based on all of the relevant parameters, namely: 1) SNR, 2) SIR, 3) interference bandwidth, and 4) interference time duration. The development of an algorithm that takes into account all of these factors is left for future work.

\subsubsection{Example: Three Receivers on Additive White Gaussian Noise Channel with Interference}

The analytic expressions for performance, e.g. Eqs. (98) and (102), were validated through Monte Carlo simulation of three receivers operating under conditions satisfying assumptions AS-1 through AS-7. The purpose of the simulation was to validate the implementation of the SDR combining code and verify that the derived analytic expressions are correct. The simulated signal contains $K=1024$ carriers of which $K_{I}=512$ are contaminated with time orthogonal interference of time duration $N_{I}=256$ samples. Two simulations, A and B, consisting of 17,520 simulated receptions at each different SNR and SIR level were performed. Simulation A held the SNR on each receiver constant at 0,3 , and $6 \mathrm{~dB}$ respectively and varied the SIR level from -10 to $10 \mathrm{~dB}$. Each receiver experienced the same SIR as it was varied. Simulation B held the SIR on each receiver constant at -3, 0 , and $3 \mathrm{~dB}$ while varying the SNR from -10 to $6 \mathrm{~dB}$. Each receiver experienced the same SNR as it was varied. Table 2 lists the simulation parameters. As part 


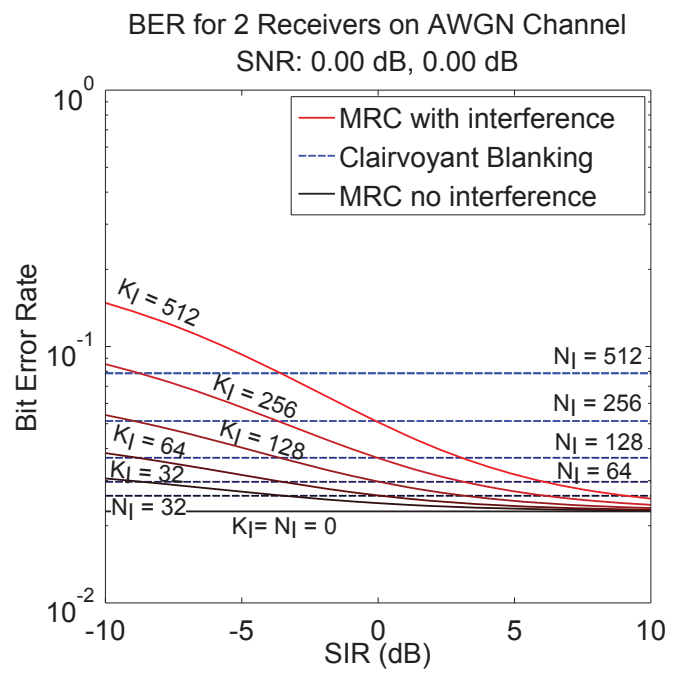

(a)

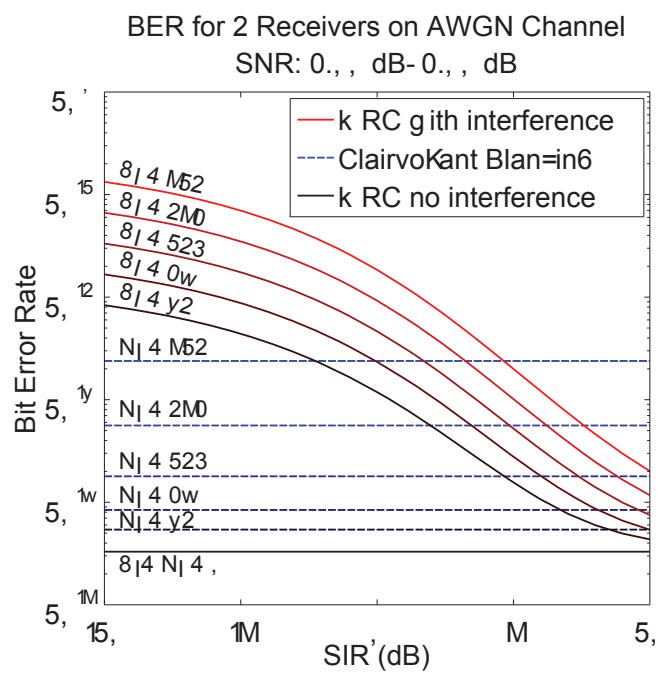

(c)

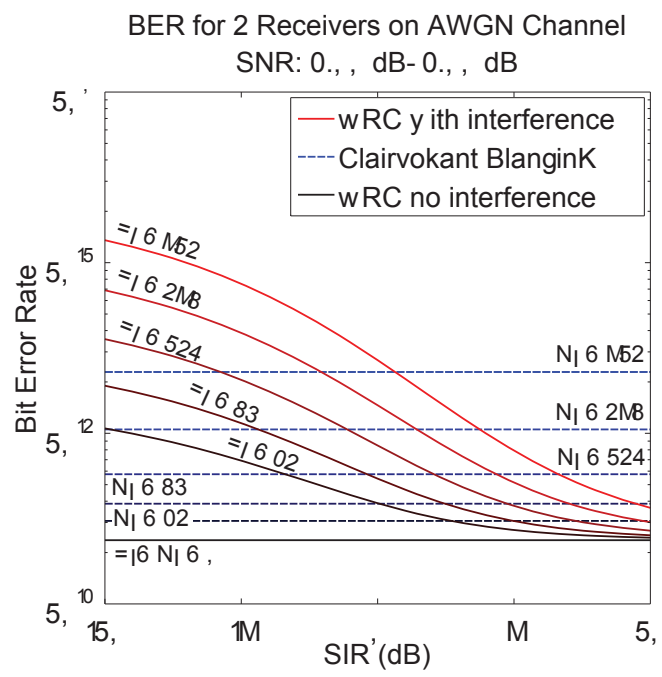

(b)

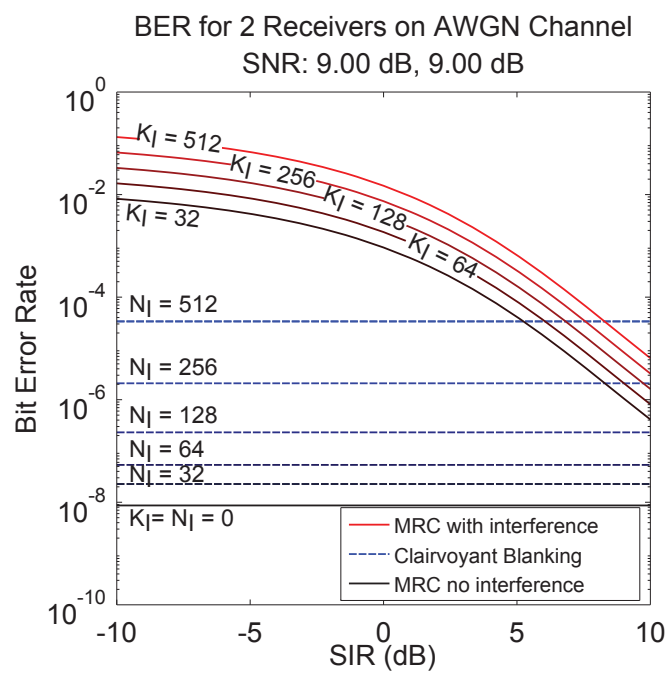

(d)

Figure 23: Performance on AWGN channel for two receivers suffering equivalent noise and interference levels.

The number of OFDM carriers is $K=1024$. The two receivers suffer the same SIR, varying from -10 to $10 \mathrm{~dB}$. The red curves are theoretical results for MRC and the blue curves are for clairvoyant blanking. The interference bandwidth varies from $3.125 \%\left(K_{I}=32\right)$ to $50 \%\left(K_{I}=\right.$ $512)$ of the band. The time duration of the interference varies similarly from $3.125 \%\left(N_{I}=32\right)$ to $50 \%\left(N_{I}=512\right)$ of the baseband symbol duration. 

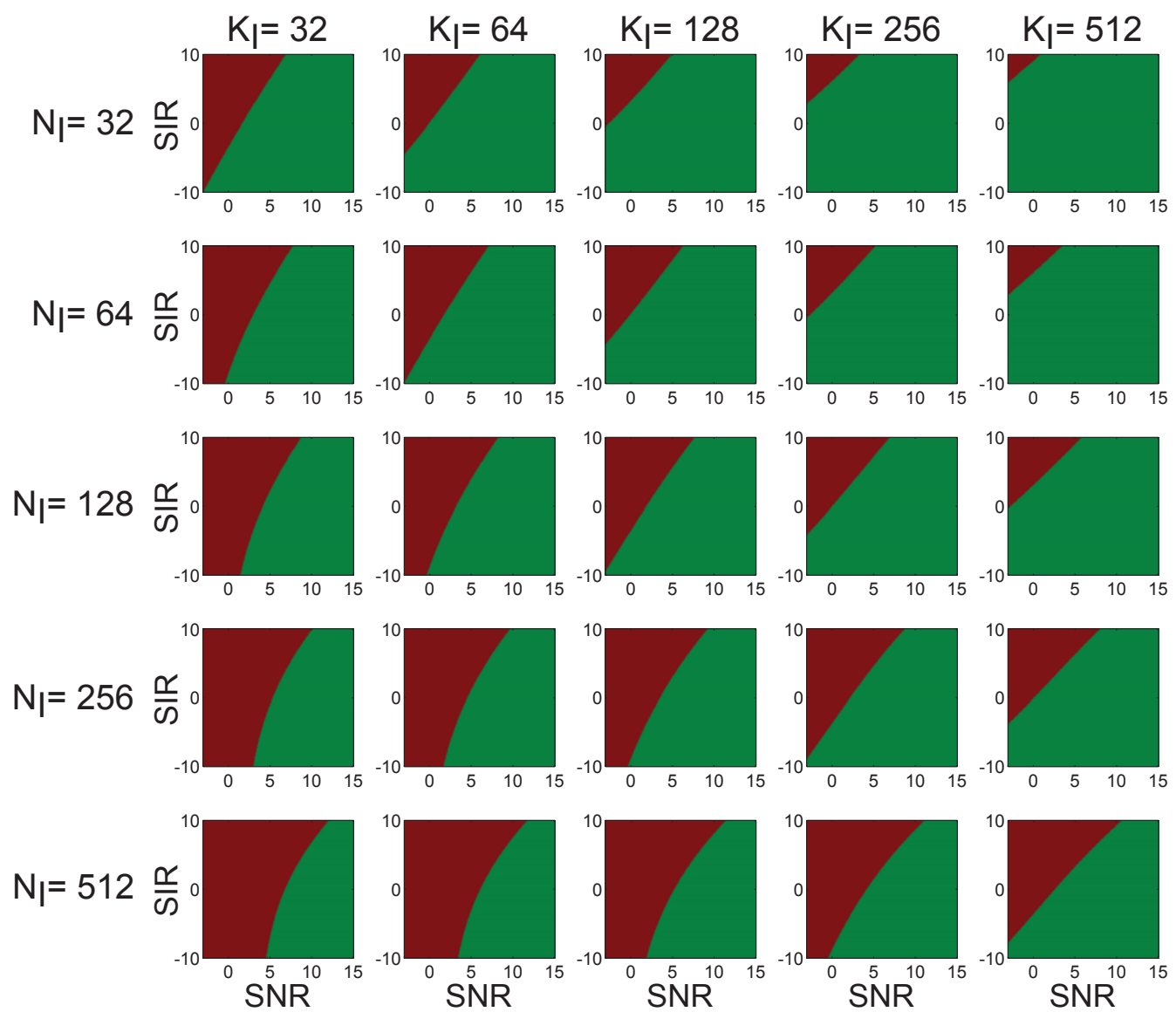

Use MRC

Use Blanking

Figure 24: Comparison of MRC and clairvoyant blanking performance for two receivers suffering equivalent noise and interference levels of various bandwidths and time durations.

The two receivers suffer the same SIR, varying from -10 to $10 \mathrm{~dB}$ and SNR varying from -3 to $15 \mathrm{~dB}$. The number of OFDM carriers is $K=$ 1024. The interference bandwidth varies from $3.125 \%\left(K_{I}=32\right)$ to $50 \%\left(K_{I}=512\right)$ of the band across the columns. The time duration of the interference varies similarly from $3.125 \%\left(N_{I}=32\right)$ to $50 \%\left(N_{I}=\right.$ 512 ) of the baseband symbol duration down the rows. Green indicates regions where the BER for blanking is lower than MRC, and hence, blanking should be employed. Red indicates regions where the BER for MRC is lower than blanking, and therefore, MRC should be used. The SDR algorithm transitions between the two combining strategies. 


\begin{tabular}{|c|c|c|}
\hline Parameter & Simulation A & Simulation B \\
\hline Number of Carriers, $K$ & 1024 & 1024 \\
\hline Number of Frequency Bins with Interference, $K_{I}$ & 512 & 512 \\
\hline Sample Duration of Interference, $N_{I}$ & 256 & 256 \\
\hline Channel 1 SNR & 0 & -6 to 6 \\
\hline Channel 2 SNR & 3 & -6 to 6 \\
\hline Channel 3 SNR & 6 & -6 to 6 \\
\hline Channel 1 SIR & -10 to 10 & -3 \\
\hline Channel 2 SIR & -10 to 10 & 0 \\
\hline Channel 3 SIR & -10 to 10 & 3 \\
\hline Number of Realizations & 17,520 & 17,520 \\
\hline Number of Simulated Bits & $17,940,480$ & $17,940,480$ \\
\hline Results Figure & 25 & 26 \\
\hline
\end{tabular}

Table 2: Parameters for simulation of AWGN channel with interference

of both simulations, the same noise data was used to simulate receptions without interference. These receptions were processed using the MRC algorithm to provide a baseline. Fig. 25 shows the results from simulation A and Fig. 26 shows the results from simulation B. The analytic expressions of performance and Monte Carlo results clearly confirm one another, validating that the SDR combining code was implemented properly and that the analytic expressions are correct.

The simulated SIRs and SNRs were chosen to demonstrate the behavior of the combining strategies, not because they represent the expected operating regime of the receiver. The following observations are made about the two algorithms based on the results in Figs. 25 and 26:

- The performance of blanking is constant with SIR since it removes the portions of the signals suffering from interference (see Fig. 25).

- For a given noise level, there is an interference level at which it is better to apply MRC instead of blanking. As noted above, this is the interference level where $\sigma_{\mathrm{MRC}_{e q}^{k}}^{2}<\sigma_{\mathrm{SDR}^{k}}^{2}$ (see Fig.25).

- For a given interference level, there is greater gain in applying blanking 


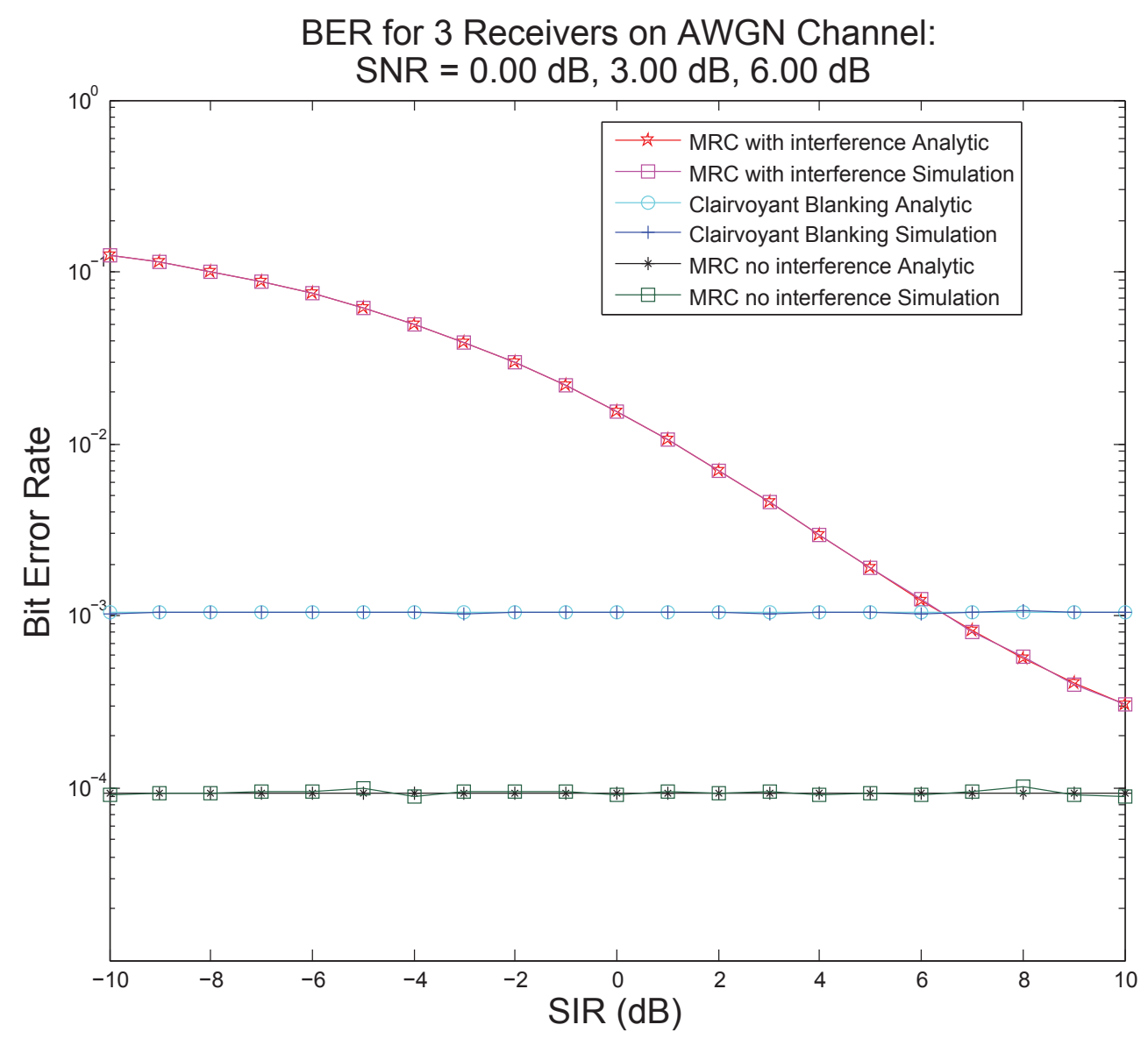

Figure 25: Performance on AWGN channel as a function of SIR. Analytic and Simulation results for different combining strategies on AWGN Channel with and without interference. Clairvoyant blanking of interference applies the SDR algorithm with exact knowledge of the samples suffering from interference. The interference is time orthogonal with a duration of 256 samples. The SNR is 0,3 , and $6 \mathrm{~dB}$ respectively for each receiver. The SIR is the same for all channels varying from -10 to $10 \mathrm{~dB}$. The range of SIRs was chosen to demonstrate the behavior of the combining strategies, not because it represents the expected operating regime of the receiver. 


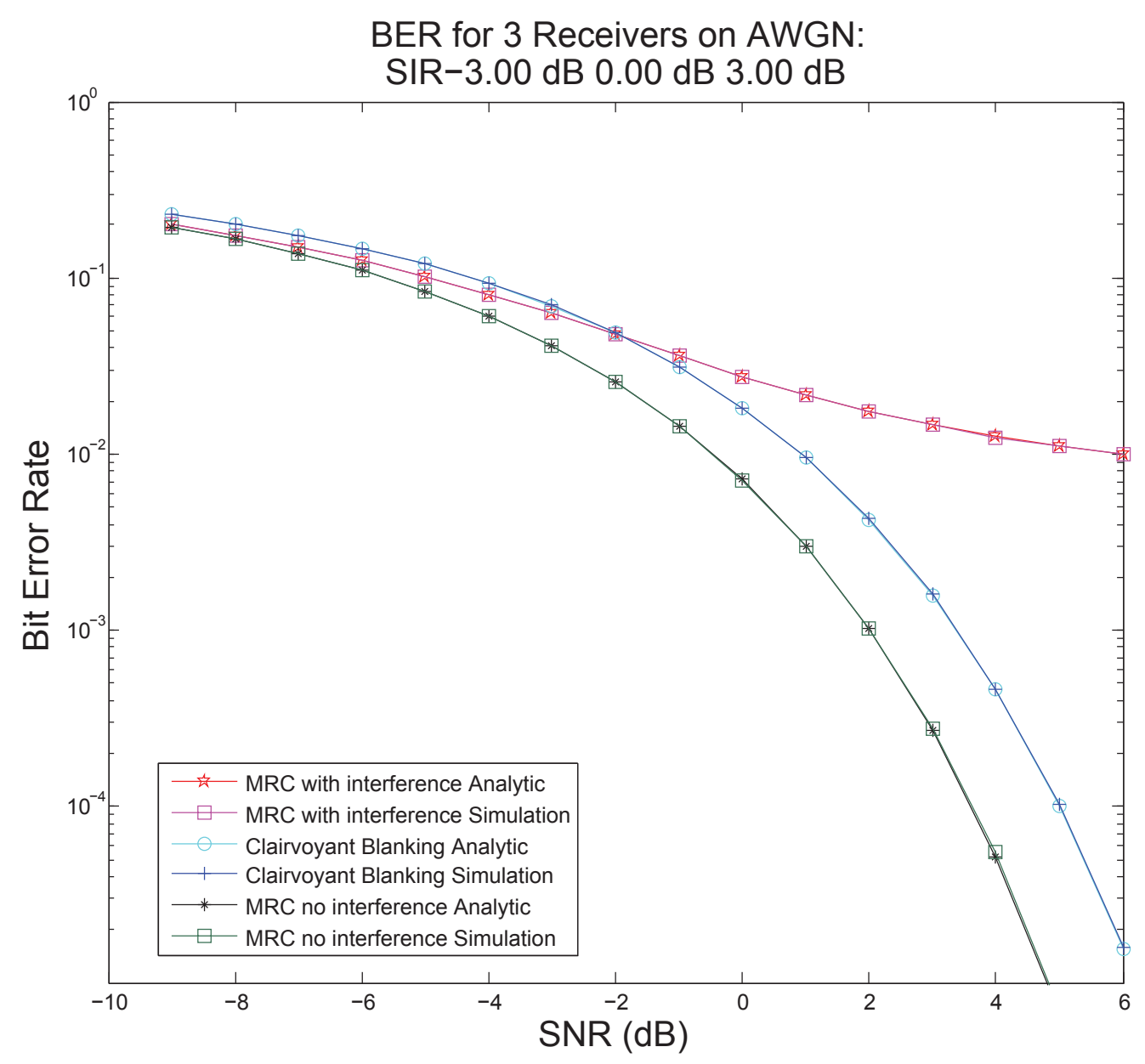

Figure 26: Performance on AWGN channel as a function of SNR. Analytic and Simulation results for different combining strategies on AWGN Channel with and without interference as a function of SNR. Clairvoyant blanking of interference applies the SDR algorithm with exact knowledge of the samples suffering from interference. The interference is time orthogonal with a duration of 256 samples. The SIR is $-3,0$ and $3 \mathrm{~dB}$ respectively for each receiver. The SNR is the same for all channels varying from -6 to $6 \mathrm{~dB}$. The range of SNRs was chosen to demonstrate the behavior of the combining strategies, not because it represents the expected operating regime of the receiver. 
instead of MRC as the SNR level increases (see Fig. 26).

- The performance of blanking on a channel suffering interference does not achieve the performance of MRC on a channel without interference. That is, blanking cannot completely mitigate all the effects of the interference (see Figs. 25 and 26).

- The performance of all the combining strategies tends to converge as SNR gets very low (see Fig. 26).

\subsection{Simulation Results on a Time Invariant Channel}

The spatial diversity reconstruction algorithm was tested on a time invariant channel using simulated data. The geometry for the simulation, shown in Fig. 27, postulated a source equidistant from two receivers and an interfering signal closer to receiver 1 than receiver 2 causing the interference to arrive at receiver 1 before receiver 2. The simulation further postulated that the interference arrives in the first half of the OFDM symbol period on receiver 1 whereas it corrupts the second half of the received signal on receiver 2. The simulated time-invariant channels are shown in Fig. 28. Channel 1 is taken from [21]. Simulation results were obtained for two cases: 1) a priori known channel impulse response in which the receptions were equalized by inverting the channel response; that is, a zero-forcing equalizer was employed; 2) unknown channel impulse response in which a minimum mean square error equalizer estimated the channel response based on pilot tones using the estimator from [32]. Table 3 lists the simulation parameters.

The interference is generated by passing white Gaussian noise of time duration $T_{I}=T / 4 \mathrm{~ms}$ through a bandpass filter with a center frequency of $15 \mathrm{kHz}$ and bandwidth of $2.4 \mathrm{kHz}$. The delay of the interference relative to the start of each block is uniformly distributed according to the start time parameter listed 


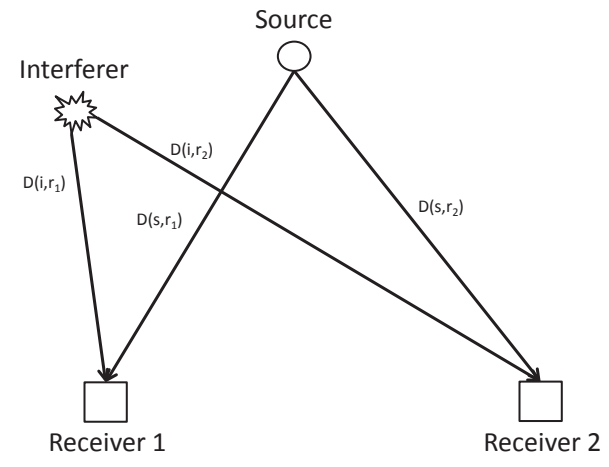

Figure 27: Simulation geometry. $D\left(s, r_{1}\right)=D\left(s, r_{2}\right)$ and $D\left(i, r_{1}\right)<D\left(i, r_{2}\right)$.

in Table 3. The interference is thus orthogonal in the time domain on the two receivers but overlaps in the frequency domain.

The simulated time domain interference is sampled, overlapped and added, and an FFT is taken to produce frequency domain interference which is then scaled to the appropriate SIR and added to the background noise, which is modeled as complex white Gaussian noise with an SNR of 7.9 dB. After adding the simulated noise to an OFDM symbol vector, the waveform reconstruction algorithm was run at an SNR of $7.9 \mathrm{~dB}$ for SIRs varying from -10 to $2 \mathrm{~dB}$. The Monte Carlo simulation was stopped when either 500,000 bits had been processed or 250 errors were made.

Fig. 29 clearly demonstrates the benefits of leveraging spatial diversity to reconstruct the transmitted waveform. The figure shows a comparison of the performance of the spatial diversity reconstruction (SDR) technique and the traditional maximum ratio combining (MRC) technique using a minimum mean square error (LS) equalizer which must estimate the channel and a zero forcing (ZF) equalizer which knows the channel a priori. The MRC performance on the same channels without interference and the single receiver performance on an additive white Gaussian noise channel with no interference are also shown for comparison. SDR consistently performs better than MRC and significantly so at low SIRs. The 

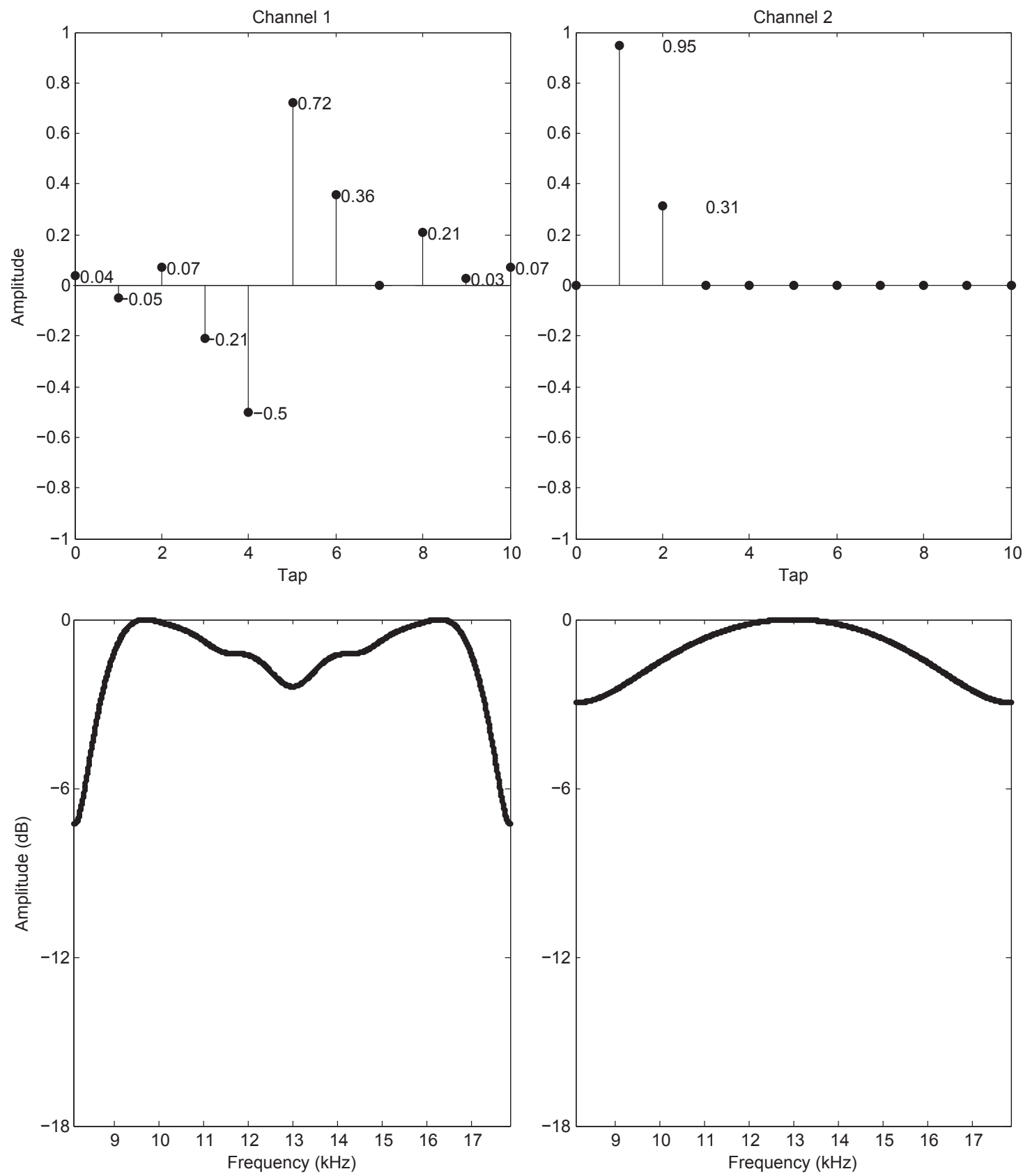

Figure 28: Simulated channels. 


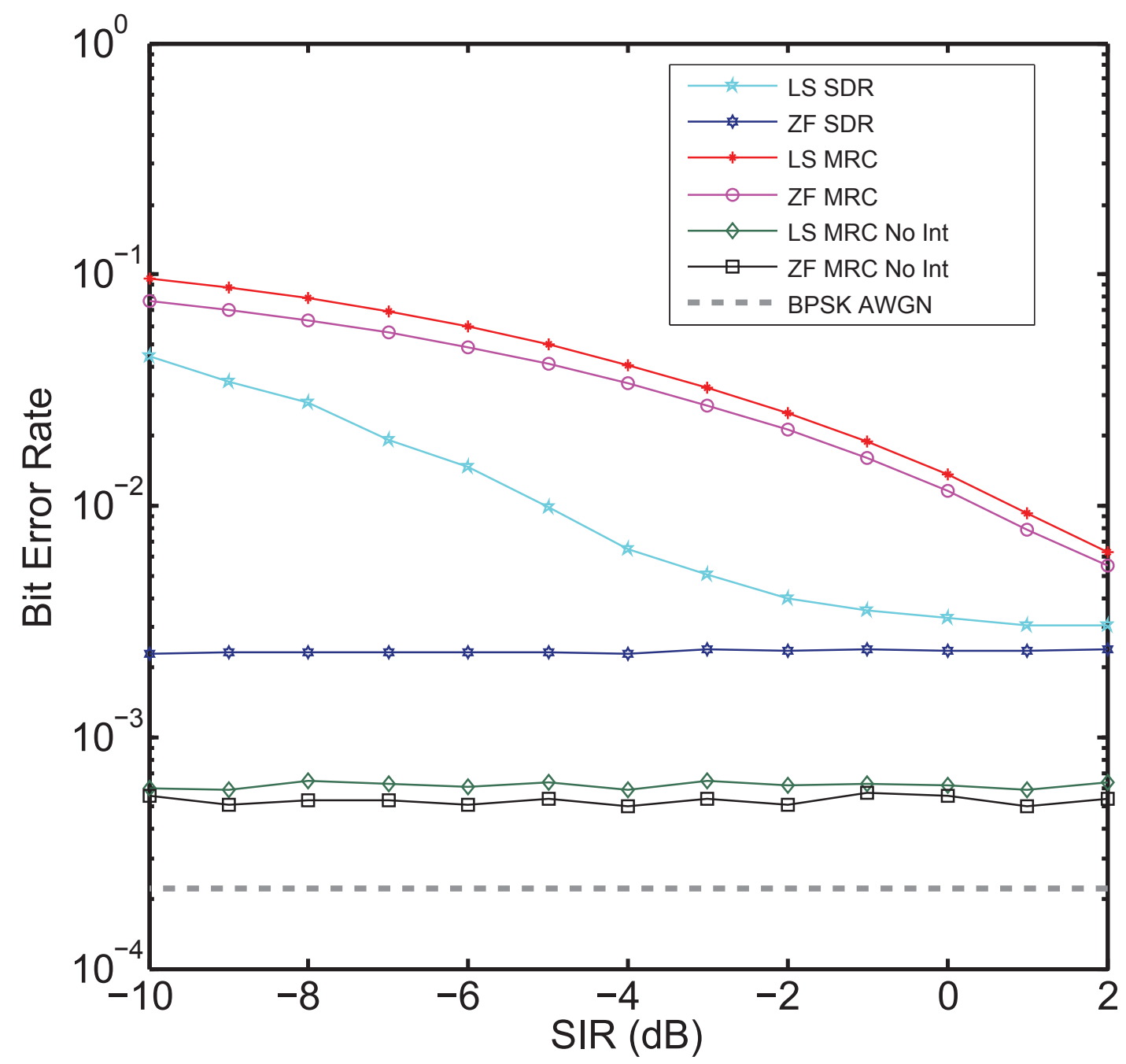

Figure 29: Two receiver performance on time invariant channel

Bit Error Rate for different combining strategies and equalization methods at an SNR of $7.9 \mathrm{~dB}$ and various SIRs: SDR - Spatial Diversity Reconstruction, MRC - Maximum Ratio Combining, No Int - No interference present, LS - Least Squares Equalizer, ZF - Zero Forcing Equalizer, BPSK AWGN - binary phase shift keying on an impulse channel in additive white Gaussian noise. 


\begin{tabular}{|l|l|l|}
\hline \multicolumn{2}{|c|}{ OFDM Parameter } & Value \\
\hline Center frequency & $f_{c}$ & $13 \mathrm{kHz}$ \\
\hline Bandwidth & $B$ & $9.77 \mathrm{kHz}$ \\
\hline \# of subcarriers & $K$ & 1024 \\
\hline \# data subcarriers & $\left|\mathcal{S}_{D}\right|$ & 672 \\
\hline \# pilot subcarriers & $\left|\mathcal{S}_{P}\right|$ & 256 \\
\hline \# null subcarriers & $\left|\mathcal{S}_{N}\right|$ & 96 \\
\hline Symbol Duration & $T$ & $104.68 \mathrm{~ms}$ \\
\hline Symbol Constellation & & BPSK \\
\hline Subcarrier spacing & $\Delta f=1 / \mathrm{T}$ & $9.54 \mathrm{~Hz}$ \\
\hline Guard interval & $T_{g}$ & $24.6 \mathrm{~ms}$ \\
\hline Number of Guard Samples & $N_{g}$ & 240 \\
\hline \multicolumn{2}{|c|}{ Interference Parameter } & Value \\
\hline Center Frequency & $f_{c, I}$ & $15 \mathrm{kHz}$ \\
\hline Bandwidth & $B_{I}$ & $2.4 \mathrm{kHz}$ \\
\hline Duration & $T_{I}$ & $26.2 \mathrm{~ms}$ \\
\hline Channel 1 Start time & $T_{s, 1}$ & $\mathcal{U}\left(.1 T_{I}, T / 2-1.1 T_{I}\right)$ \\
\hline Channel 2 Start time & $T_{s, 2}$ & $\mathcal{U}\left(T / 2+.1 T_{I}, T-1.1 T_{I}\right)$ \\
\hline
\end{tabular}

Table 3: Parameters for simulation of linear time invariant channel.

importance of accurate channel estimation and equalization is seen in noting the difference in the performance of the SDR algorithm with the LS and ZF equalizers at low SIRs. Channel equalization plays a critical role not only because better equalization improves the averaging operation in the time domain, but also because any noise enhancement resulting from equalization is smeared across the time series through the subsequent Fourier transform operation.

Fig. 30 further manifests the importance of channel equalization. Even when the channel is perfectly known to the receiver and a zero-forcing equalizer is employed, the performance does not approach that possible on an additive white Gaussian noise channel. The equalizer does not remove all the effects of the channel.

\subsection{Experimental Results}

During May 2014, experiments were conducted at AUTEC to test and compare the SDR and PIC interference mitigation algorithms. A multichannel projector was configured for multichannel simultaneous transmission of both interfering and 


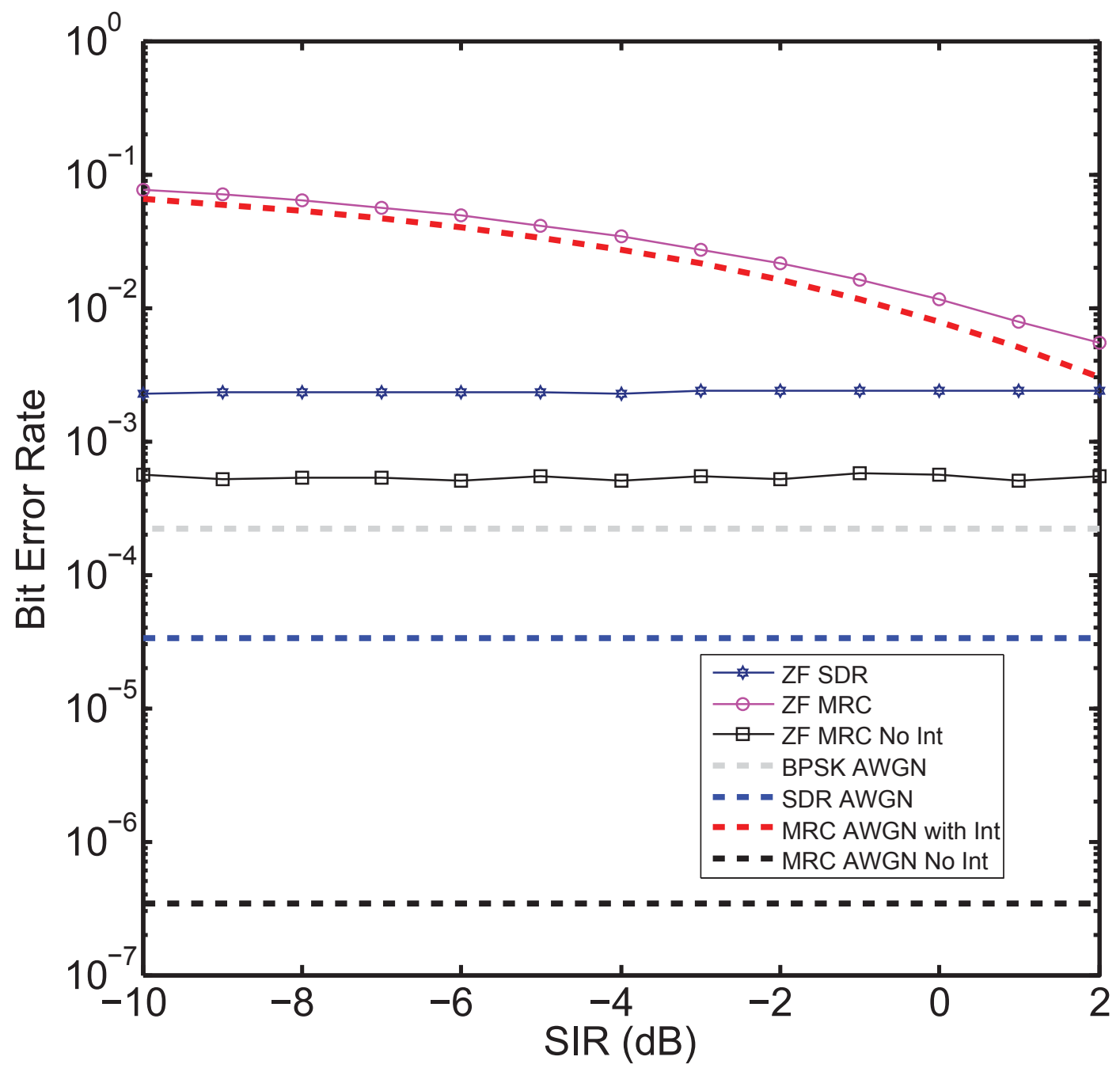

Figure 30: Performance comparison between time invariant channel and AWGN channel

Bit Error Rate for different combining strategies for two receivers using a zero-forcing (ZF) equalizer on a time invariant channel compared with performance of the same algorithms on an impulse channel with additive white Gaussian noise at various SIRs: SDR - Spatial Diversity Reconstruction, MRC - Maximum Ratio Combining, No Int - No interference present, with Int - with interference present. Performance of a single receiver on an impulsive channel with additive white Gaussian noise is also shown (BPSK AWGN). 


\begin{tabular}{|l|l|l|}
\hline \multicolumn{2}{|l|}{ OFDM Packet LFM Probe Parameters } & Value \\
\hline Center Frequency & $f_{c, P}$ & $11 \mathrm{kHz}$ \\
\hline Sweep Direction & & Down \\
\hline Bandwidth & $B_{P}$ & $6.0 \mathrm{kHz}$ \\
\hline Duration & $T_{P}$ & $100 \mathrm{~ms}$ \\
\hline \multicolumn{2}{|c|}{ OFDM Parameters } & Value \\
\hline Center frequency & $f_{c}$ & $11 \mathrm{kHz}$ \\
\hline Bandwidth & $B$ & $6.0 \mathrm{kHz}$ \\
\hline \# of subcarriers & $K$ & 1024 \\
\hline \# data subcarriers & $\mid \mathcal{S}_{D}$ & 672 \\
\hline \# pilot subcarriers & $\mathcal{S}_{P} \mid$ & 256 \\
\hline \# null subcarriers & $\left|\mathcal{S}_{N}\right|$ & 256 \\
\hline Symbol Duration & $T$ & $170.7 \mathrm{~ms}$ \\
\hline Subcarrier spacing & $\Delta f=1 / \mathrm{T}$ & $5.859 \mathrm{~Hz}$ \\
\hline Guard interval & $T_{g}$ & $250 \mathrm{~ms}$ \\
\hline Symbol Constellation & & $\mathrm{BPSK}$ \\
\hline Blocks Per Packet & $N_{b l k}$ & 4 \\
\hline Blanking Period Between Packets & & $2.9 \mathrm{sec}$ \\
\hline \multicolumn{2}{|c|}{ LFM Interference Parameters } & Value \\
\hline Center Frequency & $f_{c, I}$ & $14 \mathrm{kHz}$ \\
\hline Sweep Direction & & $\mathrm{Up}$ \\
\hline Bandwidth & $B_{I}$ & $B / 8, B / 4, B / 2$ \\
\hline Duration & $T_{I}$ & $T / 8, T / 4, T / 2$ \\
\hline Repetition Rate & $R_{I}$ & $T+T g=420.7 \mathrm{~ms}$ \\
\hline
\end{tabular}

Table 4: AUTEC-0514 OFDM and LFM transmit parameters.

desired acoustic waveforms. The desired OFDM signal consisted of a channel probe, four data packets containing identical messages and a final channel probe while Linear Frequency Modulated (LFM) waveforms of various time durations and bandwidths provided the interference. Receptions of the transmitted waveforms were recorded on more than 40 distributed ocean-bottom hydrophones.

\subsubsection{Example of SDR on Experimental Data}

Examples of the received data packets are shown in Fig 31. The interfering LFM waveform (of bandwidth $3,000 \mathrm{~Hz}$ and time duration 85 milliseconds) is clearly evident in both spectrograms, occurring on the front half of the data block on receiver 68 and on the back half of the data block on receiver 76 . The receivers are separated by approximately 4,000 yards. In addition to the LFM interference, the third and fourth data blocks suffer interblock interference (IBI) as portions of 
the surface bounce arrival of the first two data blocks coincides with the direct path arrival of these blocks.

Independently processing the third block from the channels shown in Fig. 31 without interference suppression resulted in thirteen errors on receiver 68 and eight errors on receiver 76 whereas applying the parameterized interference mitigation algorithm resulted in no errors. Processing the third blocks using maximum ratio combining $(\mathrm{MRC})$ resulted in three errors while applying spatial diversity reconstruction (SDR) resulted in no errors. While the interference mitigation techniques both reduce the BER to zero, applying the error correction code also reduces the error rate to zero. Thus, the example is not the most convincing demonstration of the power of these techniques because the interference level is not high enough to drive the error rate above the correction capability of the code. Unfortunately, the interference level in this example is the highest observed during the experiment. Consequently, in order to investigate the performance of the algorithms over a wider range of SIR and SNR levels, pseudo-experimental data was generated from actual received data.

\subsubsection{Statistical Performance of Algorithms Based on Pseudo- Experimental Data}

In order to test the performance of the algorithms at different interference and noise levels, a data set of 1,500 synthesized received signals was created from the interference and noise sampled during the experiment. The pseudo-experimental interference was created by windowing the appropriate portions of the received direct path LFM signals and amplifying them to the appropriate interference level. Similarly, noise from portions of the experiment when no signal or interference was present was amplified to the appropriate level to vary the signal to noise ratio. The amplified interference and noise was added to received OFDM blocks in which 


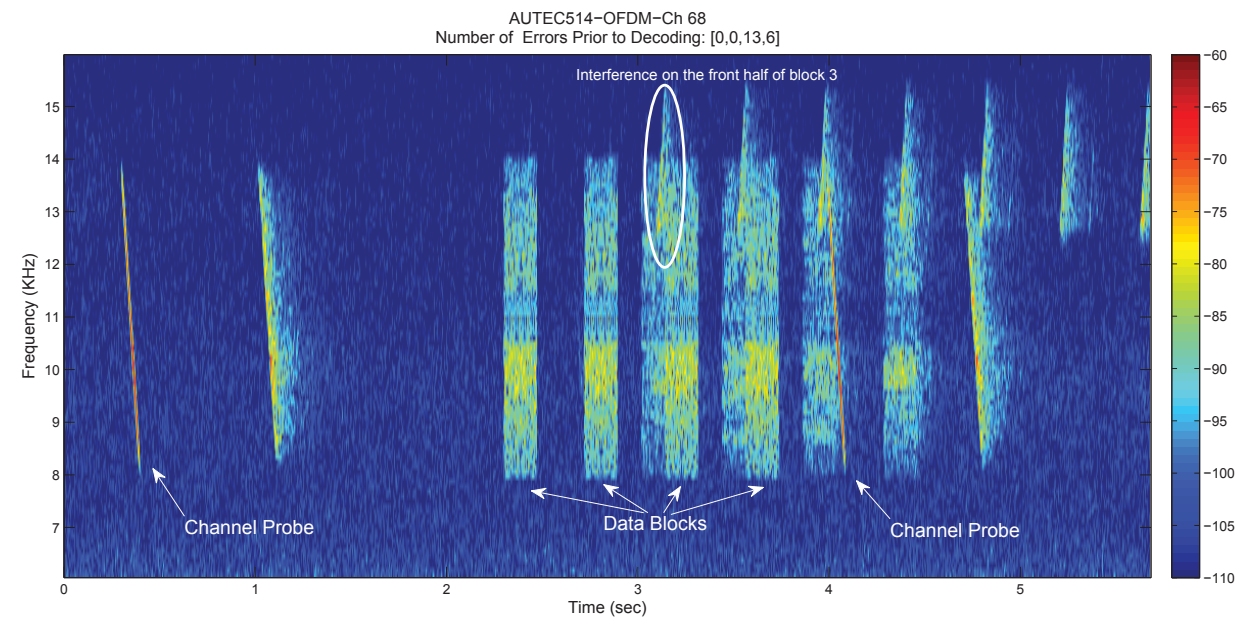

(a)

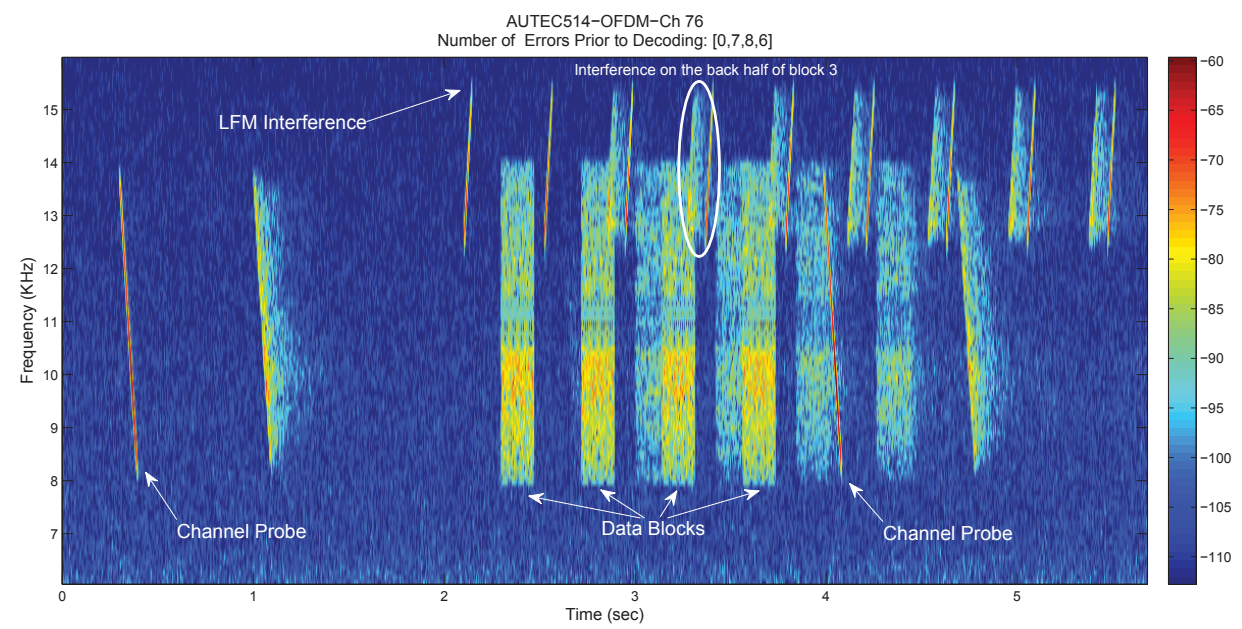

(b)

Figure 31: The OFDM signals received on channels 68 (panel a) and 76 (panel b) during AUTEC-0514.

Note that LFM interference corrupts the beginning of block 3 of the data packet on channel 68 and the end of the same block on channel 76. The receivers are separated by approximately 4,000 yards. 


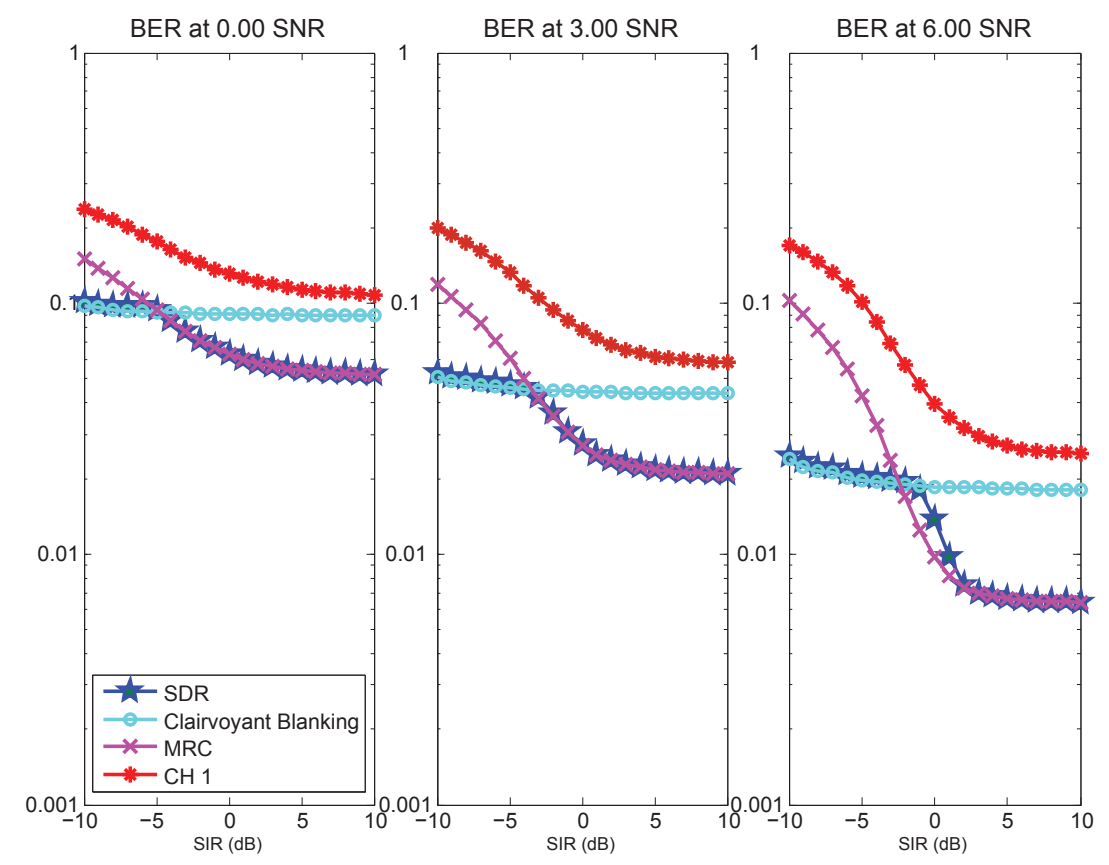

Figure 32: Uncoded bit error rate performance as a function of SIR for different mitigation strategies.

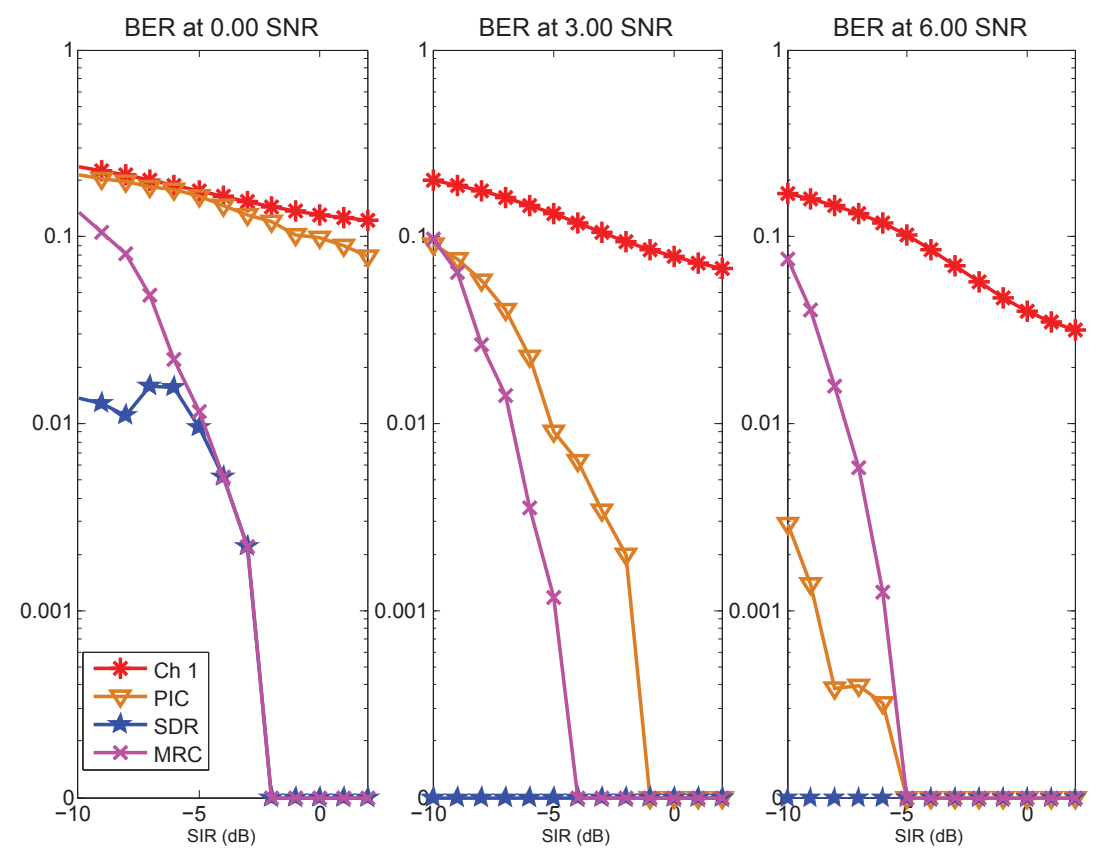

Figure 33: Coded bit error rate performance as a function of SIR for different mitigation strategies. 
the symbols were all detected correctly prior to decoding. For testing of the SDR and MRC algorithms, the interference was time orthogonal on the two receivers. A detailed explanation of the steps used to generate the pseudo-experimental data is provided in Appendix A.

The results of processing the pseudo-experimental signals prior to decoding are shown in Fig. 32 for single channel processing, MRC and SDR. Clairvoyant blanking (shown in cyan circles) always excised the correct portion of the signal suffering interference whereas the SDR algorithm (shown in blue stars) had to detect the presence of interference as well as select the portion of the received signals to excise. The SDR algorithm applied the blanking strategy when interference was high and the MRC strategy otherwise. At low SIRs (when the interference is large), there is a clear benefit to excising the interference for all values of SNR, but more dramatic gain is realized at higher SNRs as evidenced by comparing the reduction in BER seen as SNR increases from 0.00 to $6.00 \mathrm{~dB}$ for the same SIR level. For example, at $-6.00 \mathrm{~dB}$ SIR and $0.00 \mathrm{~dB}$ SNR, the BER is 0.0979 for SDR and 0.1153 for MRC; SDR performance is better by 0.0174. In comparison for the same interference level (-6.00 dB SIR) but a higher SNR of $6.00 \mathrm{~dB}$, the BER is 0.0208 for SDR and 0.0548 for MRC; SDR performance is better by 0.0340 . As the interference power approaches the noise power, the benefit of excising the interference diminishes and the loss from not averaging over the noise becomes apparent. This is demonstrated at higher SIRs in the results for clairvoyant blanking where, even with perfect knowledge of the time window suffering interference, performance is degraded as compared to MRC. These results are consistent with the theoretical results presented earlier. The interference detection threshold can be set to transition between the SDR and MRC combining strategies. The blanking strategy was employed when the significance level of the Kolmogorov-Smirnov 
hypothesis test exceeded $k s_{\text {th }}$. By only declaring interference when it is sufficiently large, the SDR algorithm exhibits the same performance as MRC at high SIRs and the benefits of the clairvoyant blanking at low SIR. Even though the threshold employed was not adjusted for SNR, the SDR algorithm achieves close to the best possible combining performance across the range of SNRs tested. At 0.00 and 3.00 SNR, the SDR algorithm closely follows the best combining method: blanking at low SIR and MRC at high SIR. At an SNR of $6.00 \mathrm{~dB}$, a small performance gap exists between SDR and MRC as the SIR approaches zero. Note that the lower the SIR, the easier it is to detect the interference and correctly remove the corrupted portion of the signal. The effectiveness of the time and frequency domain interference detectors is borne out in the experimental results. The results show that, when the algorithm is blanking the interference, its performance is very close to the clairvoyant detector as illustrated by the proximity of the SDR and clairvoyant blanking curves in Fig. 32 at low SIRs.

Fig. 33 shows the bit error rate after decoding for the various mitigation strategies. (The single channel uncoded bit error rate is also shown for comparison). The single channel PIC algorithm (orange triangles) is effective at moderate SIR levels. This is the region where Fig. 32 indicated the most effective strategy was to transition from blanking to averaging (MRC). The PIC technique is ineffective at low SIRs; precisely the region where blanking gains are most dramatic.

As discussed in the previous chapter, SDR requires more detailed prior knowledge than PIC. However, as seen in Fig. 33, SDR performance is less dependent on the SIR than PIC since PIC relies on accurately estimating the channel and transmitted symbols to aid in estimating and canceling the interference. As the interference gets stronger, channel estimation and symbol detection degrade resulting in poorer estimates of the interference. At loud enough interference levels, the 
algorithm fails. Because SDR blanks the interference rather than attempting to cancel it, the algorithm is less susceptible to interference induced errors as shown in both Figs. 32 and 33. In contrast to PIC, at $3.00 \mathrm{~dB}$ SNR or above, there is no low SIR threshold where SDR fails. Its performance is less a function of SIR and more dependent on the background noise and the degree of time orthogonality of the interference.

The two approaches are complimentary in this sense: SDR is not limited by low SIR, but it requires multiple receivers; PIC is limited at low SIR but operates on a single receiver. In an undersea network one effective multi-channel receiver strategy would be to employ PIC on receptions suffering from moderate interference and transition to the multi-channel SDR technique when PIC fails. Alternatively, the SDR strategy could be employed as the default and PIC could be attempted in cases when the lack of time orthogonality and severity of the interference make blanking or averaging ineffective operations. In these cases coherent cancellation could be attempted. Chapter 5 discusses more ideas for future work and how these strategies might be combined.

\subsection{Summary}

This chapter reported on the performance of the Spatial Diversity Reconstruction algorithm under different channel conditions. Section 3.2 derived analytic expressions for the performance of SDR and MRC on an additive white Gaussian noise channel suffering interference. The SDR and MRC expressions provide the basis for the development of an adaptive interference mitigation receiver. Section 3.3 reported simulation results for the SDR and MRC algorithms operating on a linear time invariant channel. In section 3.4, the results from an experiment conducted at AUTEC in May 2014 demonstrate that both the SDR and PIC techniques are effective mitigation strategies. PIC is most effective at moderate SIRs 
whereas spatial diversity reconstruction is effective and realizes the most gain at low SIRs. The two approaches are complimentary and an effective multi-channel receiver strategy would be to adaptively utilize both techniques. 


\section{CHAPTER 4}

\section{Interference Experiment at AUTEC December 2014}

\subsection{Introduction}

This chapter describes the planning for the experiment conducted at AUTEC in December 2014, AUTEC-1214. Planning for AUTEC-1214 leveraged lessons learned and data gathered during an experiment conducted at AUTEC in May 2014, AUTEC-0514. Section 4.2 describes the objectives of the experiment and the data collection required to support the objectives. Section 4.3 explains the procedure for conducting the experiment which involves transmitting communication and interfering signals simultaneously and recording the received waveforms. Section 4.3.1 details the selection of the communication and interfering signal parameters. A simple channel model developed from the data collected during AUTEC-0514 aided in the selection of the signal parameters. Section 4.5 describes the channel model, discusses how it was developed and provides a some physical background justifying its use.

\subsection{Experiment Objectives}

Many communications signals suffer from interference which is neither impulsive nor narrowband. However, some of the interfering waveform parameters such as its bandwidth or time duration are known a priori. Such interfering waveforms may be termed structured acoustic interference (SAI). The goal of the AUTEC1214 experiment was to assess the performance of the Spatial Diversity Reconstruction (SDR) and Parameterized Interference Cancellation (PIC) algorithms in recovering messages transmitted using OFDM in the presence of SAI in an undersea environment. The objectives of the experiment are the following:

EO-1 Demonstrate the effectiveness of the Spatial Diversity Reconstruction 
(SDR) and Parameterized Interference Cancellation (PIC) algorithms in mitigating the effects of interference of known time duration and bandwidth on communication signals in undersea conditions.

EO-2 Characterize the performance of the SDR and PIC algorithms as a function of SIR and SNR.

EO2.1 Characterize the performance of the SDR and PIC algorithms for a variety of interference bandwidths and time durations.

EO2.2 Characterize the performance of the SDR as a function of the time orthogonality of the received interference.

EO-1 requires the SDR and PIC algorithms to successfully decode received OFDM signals suffering from SAI which are not decodable by other methods. In other words, the number of bit errors prior to decoding is too high for the error correction code to correct. Additionally, the SDR and PIC algorithms should not degrade the receiver performance when interference is not present. Following the work in [17], the transmitted message was encoded with a half rate LDPC code. Since [41] observed that typically no decoding errors occurred whenever the uncoded BER was below 0.1, demonstrating the effectiveness of the interference mitigation strategies requires generating received data sets with BERs greater than 0.1.

In order to satisfy EO-2, the data collected during the experiment must contain multiple receptions of the same OFDM waveform corrupted by partial-band, partial-block duration SAI over a range of SIR and SNR levels. Linear Frequency Modulated (LFM) signals of varying time durations and bandwidths were selected for use as the SAI because of their prevalent use in active SONAR applications. Since the SDR algorithm is inherently a multi-receiver algorithm, EO-2 requires multiple receptions of the same OFDM waveform corrupted by the same partial- 
band, partial-block duration interference at different times. EO-2 requires a data set of received OFDM signals corrupted by interference parameterized by:

EO-2-P1 SIR

EO-2-P2 SNR

EO-2-P3 Interference bandwidth

EO-2-P4 Interference duration

EO-2-P5 Time orthogonality of the interference

The SIR and SNR of the received signal are functions of the transmit power of the signal and interference as well as being highly dependent on the background ocean noise and the propagation of sound from transmitter to receiver. Clearly, the latter two quantities are not within the control of the experiment. Background noise level fluctuates due to changing weather conditions (such thunderstorms) and shipping traffic. Since the received power is strongly dependent on the range from the transmitter to the receiver and the AUTEC network has significant spatial extent, the experiment design opted to transmit at maximum power and rely on the variation in range from the transmitters to receivers to facilitate the collection of data at various SIR and SNR rather than varying the transmit power. The transmit power was not varied during the AUTEC-0514 experiment and receptions occurred at various SNRs as evidenced by the spectrograms and time series of the received data packets shown in Fig 34. The scaling in all the panels is the same and the relative positions of the receivers is shown in Fig. 35. The spectrogram for each of the five receivers, $(61,67,68,75,78)$, is shown above the corresponding time series. An example of the variation in SNR is seen by comparing the reception on receiver 61 shown in panel (a) with the reception on receiver 68 shown in panel 
(c). The signal is not apparent in the time series from receiver 61 whereas the data blocks are easily distinguished from the background noise in the time series from receiver 68. Another example of the variation is provided by comparing the spectrogram of the reception on receiver 78 shown in panel (e) with the other spectrograms. The data blocks are barely discernible on receiver 78 shown in panel (e) as compared to the spectrograms of the other receivers. The source of the spectral line at approximately 9 and $13 \mathrm{kHz}$ on receivers 61 and 78 is unknown. The time orthogonality of the received interference is a function of the time difference in arrival paths from the interference transmitter and the signal transmitter and the time duration of the interference. Using the channel model developed from the AUTEC-0514 data, a range of interference bandwidths, time durations and repetition rates was jointly selected so that the blocks suffering from interference would be reasonably likely to have uncoded BERs greater than 0.1. Interference bandwidths of one quarter, one half and three quarters of the OFDM signal bandwidth $\left(B_{I}=[B / 4, B / 2,3 B / 4]\right)$ in conjunction with interference time durations of one eighth, one quarter and one half of the OFDM symbol duration $\left(T_{I}=[T / 8, T / 4, T / 2]\right)$ transmitted at a repetition rate of 1.25 times the OFDM symbol duration $T$ satisfy the BER criteria.

Ideally the performance of the algorithms should be assessed directly on the received data without the need to create "pseudo-experimental" data by adding background noise or interfering signals received at other times during the experiment. However, should such "cutting and pasting" be necessary, the setup of the experiment should make it as easy as possible.

\subsection{Experiment Procedure}

Nine stereo wavefiles containing different LFM interfering signals on the left channel and the same OFDM data packets on the right channel and were created 


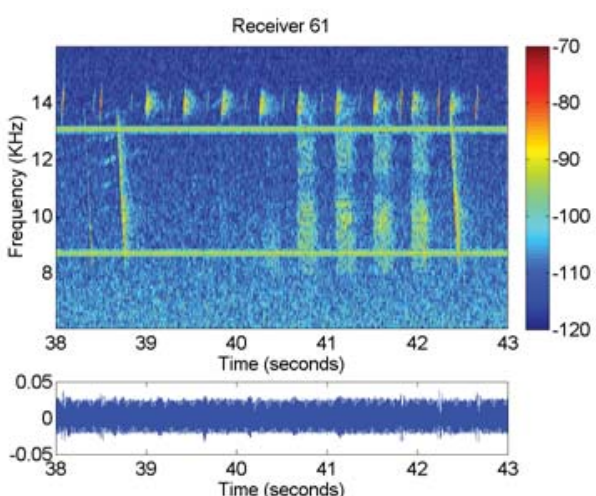

(a)
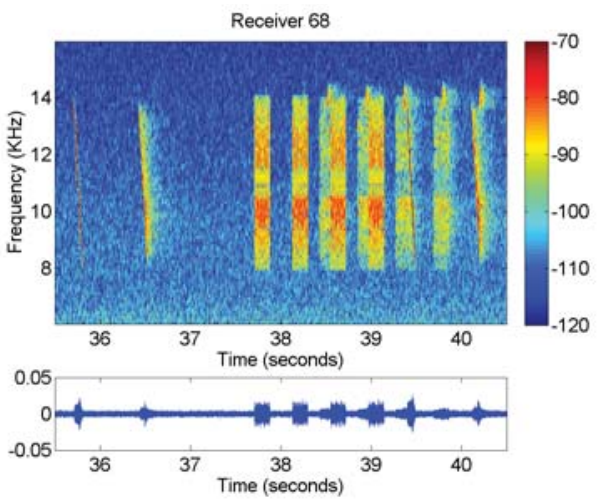

(c)

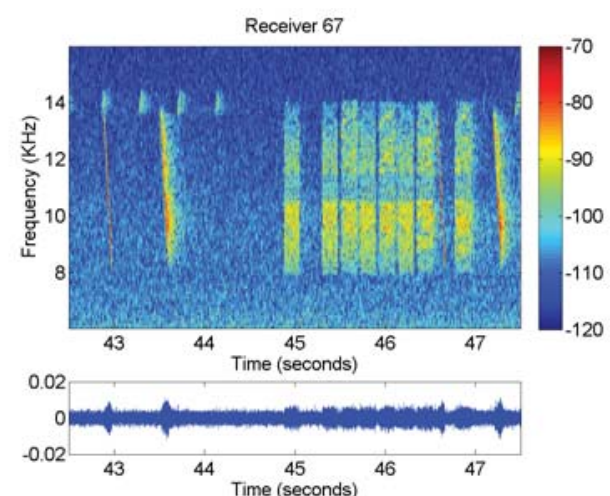

(b)

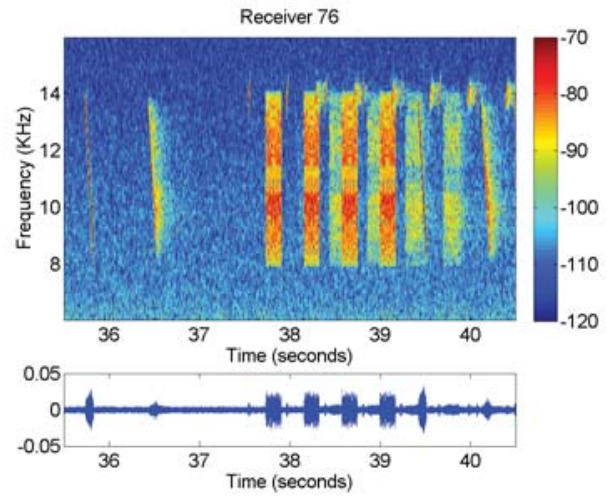

(d)

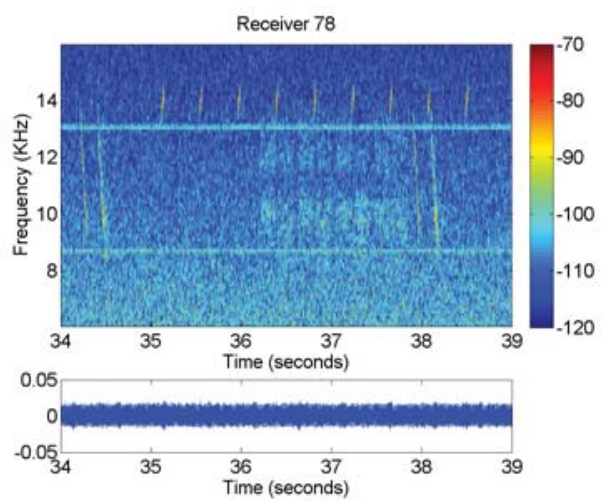

(e)

Figure 34: Spectrograms and time series of receptions from five different receivers recorded during the May experiment (AUTEC-0514).

The scaling in all the panels is the same. The variation in received SNR is readily apparent by comparing the colors of the received data blocks in the spectrograms and the amplitude of the data blocks in the time series shown below each spectrogram. 


\begin{tabular}{|l|l|l|}
\hline Receiver & \multicolumn{2}{|c|}{ Distance to Transmitter (meters) } \\
\hline & 69 & 75 \\
\hline 61 & 4,900 & 7,738 \\
\hline 62 & 4,551 & 10,115 \\
\hline $67^{*}$ & 7,876 & 4,591 \\
\hline $68^{*}$ & 4,020 & 3,858 \\
\hline 70 & 4,086 & 10,039 \\
\hline 74 & 9,467 & 3,715 \\
\hline $76^{*}$ & 3,607 & 3,896 \\
\hline $77^{*}$ & 4,294 & 7,760 \\
\hline $78^{*}$ & 7,310 & 11,593 \\
\hline 81 & 12,192 & 6,044 \\
\hline $82^{*}$ & 9,351 & 3,806 \\
\hline
\end{tabular}

Table 5: Distance from receivers to transmitters 69 and 75. Data recorded on receivers indicated with an asterisk $(*)$ aided in constructing the channel model for planning the December experiment.

using MATLAB ${ }^{\circledR}$.

The experiment consists of transmitting an interfering signal and a desired signal from two different nodes on the AUTEC range and recording the signals received on the other nodes. The signals are transmitted by playing the stereo wavefiles on a compact disc with the left and right output sent to the desired transmit nodes. For this experiment, the left channel contained the interference and the right channel had the desired signal. The data is recorded at $96 \mathrm{kHz}$ on Alesis HD24XR 24-Track recorders and transferred to external hard drives as wavefiles which can then be read into MATLAB ${ }^{\circledR}$ for data analysis. The AUTEC range limits the play time of a single $\mathrm{CD}$ to 30 minutes.

The transmitters and receivers used in the test are shown in Fig. 35. Bidirectional (transmit and receive) node 75 was selected to transmit the desired signal while node 69 was selected to transmit interference. Nodes 75 and 69 were selected as the transmit nodes because they were used in the May 2014 experiment. The distance between the transmit nodes and the receive nodes is shown in Table 5 . 


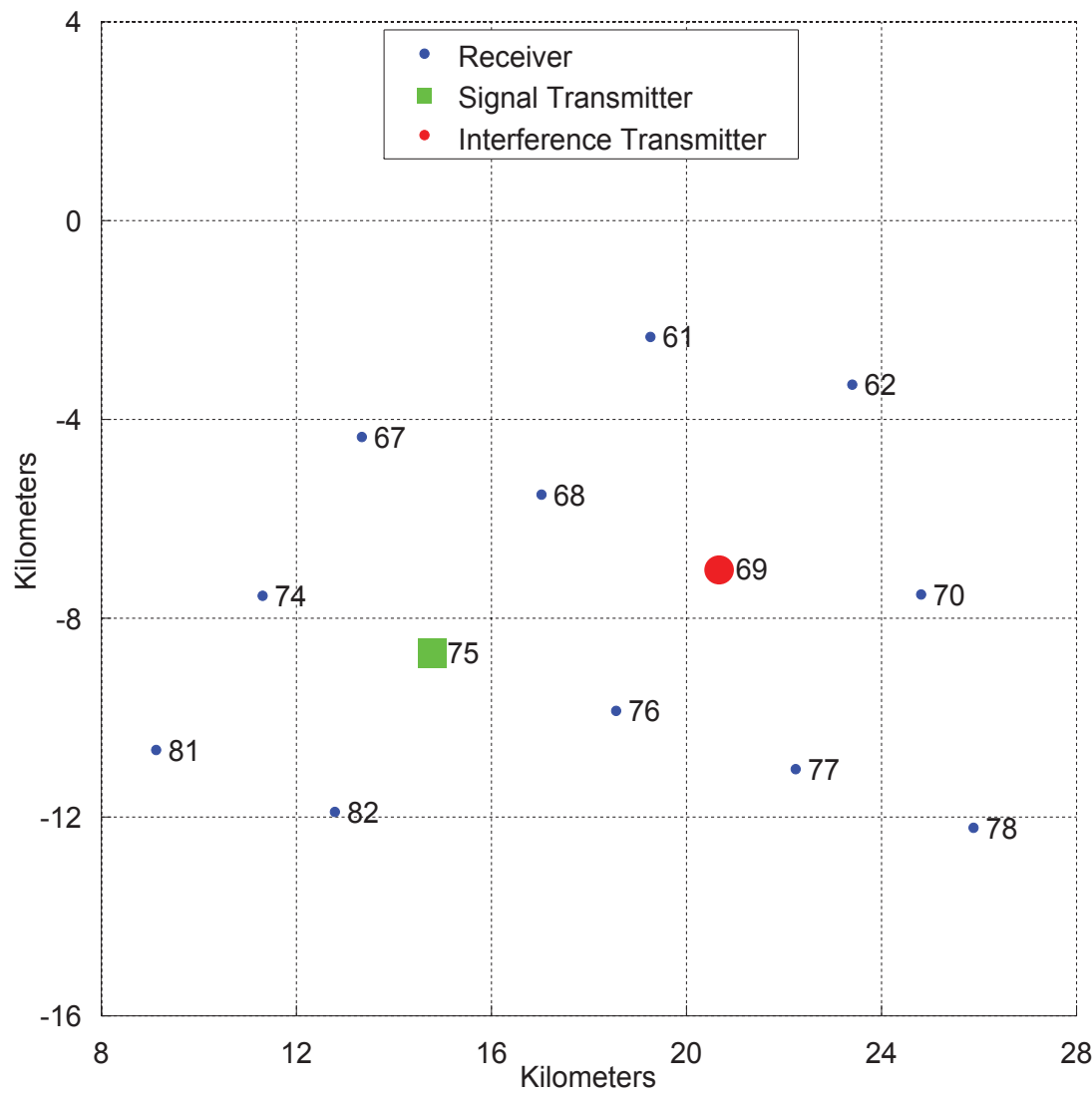

Figure 35: Relative position of transmitters and receivers in AUTEC experiments. 


\subsubsection{OFDM Communication Signal and LFM Interference}

Nine stereo wavefiles containing the same OFDM data packets on the right channel and different LFM interfering signals on the left channel were created using MATLAB ${ }^{\circledR}$. The nine files (or songs) make up the CD played during the experiment; Table 6 lists its contents. Each song is divided into 45 transmit time slots of four seconds duration. During the first two time slots, only the communication signal is transmitted. The third time slot is reserved solely for the transmission of the interfering signal. Thus, the first three time slots enable the reception of communication signals under noise only conditions and the reception of the interfering waveform without the communication signal. This was done to facilitate the post-experiment construction of received signals at various SNR and SIR levels should the desired range of levels not be present in the data recorded during the experiment. Starting with the fourth time slot, both the communication and interfering signals are transmitted.

The OFDM data packet consists of an initial channel probe, a channel probe guard period, four OFDM data blocks and associated guard intervals, a final channel probe, and a three second blanking period. The channel probes are 100 millisecond LFM signals sweeping down from $14 \mathrm{kHz}$ to $8 \mathrm{kHz}$. Each OFDM block is identical, containing the same data message encoded with a half rate binary LDPC code. The message and parity check bits are interleaved over the data subcarriers. The interference consists of upswept LFM signals.

The transmitted wavefiles are expected to generate the four types of interference listed below:

- Surface bounce receptions of the channel probe (Probe $S B$ ).

- Direct path receptions of the transmitted LFM interference (LFMDP).

- Surface bounce receptions of the transmitted LFM interference ( $L F M S B)$. 


\begin{tabular}{|c|c|c|c|c|c|}
\hline Track & File name & Time & \multicolumn{2}{|c|}{$\begin{array}{c}\text { Interference } \\
\text { (M:SS) }\end{array}$} & $\begin{array}{c}\text { Bumber of } \\
\text { Packets }\end{array}$ \\
\hline 1 & dec_li_lfm_1500BW_21Tmsec_int_li_ofdm & $3: 00$ & $1500 \mathrm{kHz}$ & $21 \mathrm{~ms}$ & 44 \\
2 & dec_li_lfm_1500BW_43Tmsec_int_li_ofdm & $3: 00$ & $1500 \mathrm{kHz}$ & $43 \mathrm{~ms}$ & 44 \\
3 & dec_li_lfm_1500BW_85Tmsec_int_li_ofdm & $3: 00$ & $1500 \mathrm{kHz}$ & $85 \mathrm{~ms}$ & 44 \\
4 & dec_li_lfm_3000BW_21Tmsec_int_li_ofdm & $3: 00$ & $3000 \mathrm{kHz}$ & $21 \mathrm{~ms}$ & 44 \\
5 & dec_li_lfm_3000BW_43Tmsec_int_li_ofdm & $3: 00$ & $3000 \mathrm{kHz}$ & $43 \mathrm{~ms}$ & 44 \\
6 & dec_li_lfm_3000BW_85Tmsec_int_li_ofdm & $3: 00$ & $3000 \mathrm{kHz}$ & $85 \mathrm{~ms}$ & 44 \\
7 & dec_li_lfm_4500BW_21Tmsec_int_li_ofdm & $3: 00$ & $4500 \mathrm{kHz}$ & $21 \mathrm{~ms}$ & 44 \\
8 & dec_li_lfm_4500BW_43Tmsec_int_li_ofdm & $3: 00$ & $4500 \mathrm{kHz}$ & $43 \mathrm{~ms}$ & 44 \\
9 & dec_li_lfm_4500BW_85Tmsec_int_li_ofdm & $3: 00$ & $4500 \mathrm{kHz}$ & $85 \mathrm{~ms}$ & 44 \\
\hline
\end{tabular}

Table 6: OFDM signal with LFM interference wavefiles.

- Inter-block interference (IBI) resulting from multipath receptions of previously transmitted OFDM blocks.

The interference expected in the received OFDM blocks for various receivers is shown in table 7.

The selection of the OFDM signal parameters was based on the following (see Table 8 for a summary):

- The packet probe was successfully used in AUTEC-0514 for packet synchronization. There was no compelling reason to modify it.

- The center frequency is driven by transmit voltage response of the AUTEC transducers. The center frequency is identical to the AUTEC-0514 experiment and the AUTEC-10 experiment reported in [17].

- Number and allocation of subcarriers matched those for the simulation results reported in [17] and the simulations conducted prior to the AUTEC-0514 experiment. The uniform distribution of pilot subcarriers enables the use of the Doppler compensation algorithm of [32] which was implemented and tested on simulated data prior to the AUTEC-0514 experiment.

- Frequency spacing $(\Delta f)$, symbol duration $(T)$, number of subcarriers $(K)$ and bandwidth $(B)$ are all related parameters $(\Delta f=1 / T, B=K \Delta f)$. 


\begin{tabular}{|l|l|l|l|l|}
\hline \multirow{2}{*}{ Receiver } & \multicolumn{4}{|c|}{ Interference } \\
\cline { 2 - 5 } & Block 1 & Block 2 & Block 3 & Block 4 \\
\hline 61 & LFM (DP\&SB) & $\begin{array}{l}\text { LFM (DP\&SB), } \\
\text { Probe (SB) }\end{array}$ & $\begin{array}{l}\text { LFM (DP\&SB), } \\
\text { Probe (SB), IBI }\end{array}$ & $\begin{array}{l}\text { LFM (DP\&SB), } \\
\text { Probe (SB), IBI }\end{array}$ \\
\hline 62 & $\begin{array}{l}\text { LFM (DP\&SB), } \\
\text { Probe (SB) }\end{array}$ & $\begin{array}{l}\text { LFM (DP\&SB), } \\
\text { Probe (SB), IBI }\end{array}$ & $\begin{array}{l}\text { LFM (DP\&SB), } \\
\text { Probe (SB), IBI }\end{array}$ & $\begin{array}{l}\text { LFM (DP\&SB), } \\
\text { Probe (SB), IBI }\end{array}$ \\
\hline $67^{*}$ & LFM (DP\&SB) & LFM (DP\&SB) & $\begin{array}{l}\text { LFM (DP\&SB), } \\
\text { Probe (SB) }\end{array}$ & $\begin{array}{l}\text { LFM (DP\&SB), } \\
\text { Probe (SB), IBI }\end{array}$ \\
\hline 70 & LFM (DP\&SB) & LFM (DP\&SB) & $\begin{array}{l}\text { LFM (DP\&SB), } \\
\text { Probe (SB) }\end{array}$ & $\begin{array}{l}\text { LFM (DP\&SB), } \\
\text { Probe (SB), IBI }\end{array}$ \\
\hline 74 & LFM (DP\&SB) & LFM (DP\&SB) & LFM (DP\&SB) & $\begin{array}{l}\text { LFM (DP\&SB), } \\
\text { Probe (SB) }\end{array}$ \\
\hline $76 *$ & LFM (DP\&SB) & LFM (DP\&SB) & LFM (DP\&SB) & $\begin{array}{l}\text { LFM (DP\&SB), } \\
\text { Probe (SB) }\end{array}$ \\
\hline 77 & LFM (DP\&SB) & $\begin{array}{l}\text { LFM (DP\&SB), } \\
\text { Probe (SB) }\end{array}$ & $\begin{array}{l}\text { LFM (DP\&SB), } \\
\text { Probe (SB), IBI }\end{array}$ & $\begin{array}{l}\text { LFM (DP\&SB), } \\
\text { Probe (SB), IBI }\end{array}$ \\
\hline $77^{*}$ & $\begin{array}{l}\text { LFM (DP\&SB), } \\
\text { Probe (SB) }\end{array}$ & $\begin{array}{l}\text { LFM (DP\&SB), } \\
\text { Probe (SB), IBI }\end{array}$ & $\begin{array}{l}\text { LFM (DP\&SB), } \\
\text { Probe (SB), IBI }\end{array}$ & $\begin{array}{l}\text { LFM (DP\&SB), } \\
\text { Probe (SB), IBI }\end{array}$ \\
\hline 81 & LFM (DP\&SB) & $\begin{array}{l}\text { LFM (DP\&SB), } \\
\text { Probe (SB) }\end{array}$ & $\begin{array}{l}\text { LFM (DP\&SB), } \\
\text { Probe (SB), IBI }\end{array}$ & $\begin{array}{l}\text { LFM (DP\&SB), } \\
\text { Probe (SB), IBI }\end{array}$ \\
\hline $82^{*}$ & LFM (DP\&SB) & LFM (DP\&SB) & LFM (DP\&SB) & $\begin{array}{l}\text { LFM (DP\&SB), } \\
\text { Probe (SB) }\end{array}$ \\
\hline
\end{tabular}

Table 7: Type of interference expected on each block for various receivers. Data recorded on receivers indicated with an asterisk $(*)$ aided in constructing the channel model for planning the December experiment. 
The frequency spacing ensures relatively flat fading on each subchannel and matches the value used in the AUTEC-10 experiment. Selecting the number of subcarriers and the frequency spacing set the bandwidth of $6 \mathrm{kHz}$. The bandwidth of the transmit signal falls within a relatively flat region of the transducer response (less than $2 \mathrm{~dB}$ variation).

- The guard interval and the number of blocks per packet were selected jointly. The guard interval of 10.0 milliseconds is more than twenty times the direct path channel length observed during the AUTEC-0514. The use of a shorter guard interval results in the OFDM data blocks being more tightly packed within the packet and a shorter overall transmit time for the packet. Consequently, more packets can be transmitted during the experiment. Based on the channel model, the fourth OFDM block is likely to suffer from surface bounce interference from the channel probe on receivers less than 5,000 meters from the OFDM transmitter. This is not detrimental to the experiment objectives since the channel probe acts as an additional interfering source. On more distant receivers, earlier blocks suffer from inter-block interference as can be seen in Table 7 . Blocks added to the packet after the fourth block would suffer from inter-block interference on all the receivers. Since mitigation of inter-block interference is not an objective of the experiment, the number of blocks in a packet was limited to four.

- The symbol constellation was chosen because it is the simplest to implement. The mapping from the binary LDPC code to the symbol constellation is straightforward.

- The three second blanking period between packets is more than twice the expected time difference of arrival between the direct path and surface bounce 
receptions. It ensures that the multipath receptions of the previously transmitted packet clears the channel before the next packet is transmitted.

The selection of the LFM interference parameters was based on the following (see Table 8 for a summary):

- The center frequency was selected to match the center frequency of the OFDM signal so that the calculation of the degree of frequency overlap between the signal and interference is obvious.

- Sweep direction. The interference and channel probe sweep in opposite directions to minimize the correlation of these signals which facilitates synchronization of the received OFDM data packets.

- Start time of first interference transmission was selected to enable the first two OFDM data packets to be received without suffering interference.

- The bandwidth, duration and repetition rate were selected jointly to meet the following criteria:

- Produce an uncoded bit error rate of greater than 0.10 on blocks suffering from interference.

- Produce received signals in which the same interfering signal (e.g. LFM signal with the same bandwidth and duration) corrupts different time domain portions of the OFDM block.

The channel model was used as a tool to aid in selecting the signal parameters discussed above. Some of the data generated using the channel model and analyzed prior to the experiment are shown in Figs. 36 and 37. As shown in Fig. 36, the uncoded BER resulting from interference in the absence of noise ranges from approximately 0.06 to 0.20 . Based on these results, the experiment is likely to 


\begin{tabular}{|l|l|l|}
\hline OFDM Packet LFM Probe Parameters & Value \\
\hline Center Frequency & $f_{c, P}$ & $11 \mathrm{kHz}$ \\
\hline Sweep Direction & & Down \\
\hline Bandwidth & $B_{P}$ & $6.0 \mathrm{kHz}$ \\
\hline Duration & $T_{P}$ & $100 \mathrm{~ms}$ \\
\hline \multicolumn{2}{|c|}{ OFDM Parameters } & Value \\
\hline Center frequency & $f_{c}$ & $11 \mathrm{kHz}$ \\
\hline Bandwidth & $B$ & $6.0 \mathrm{kHz}$ \\
\hline \# of subcarriers & $K$ & 1024 \\
\hline \# data subcarriers & $\left|\mathcal{S}_{D}\right|$ & 672 \\
\hline \# pilot subcarriers & $\left|\mathcal{S}_{P}\right|$ & 256 \\
\hline \# null subcarriers & $\left|\mathcal{S}_{N}\right|$ & 256 \\
\hline Symbol Duration & $T$ & $170.7 \mathrm{~ms}$ \\
\hline Subcarrier spacing & $\Delta f=1 / \mathrm{T}$ & $5.859 \mathrm{~Hz}$ \\
\hline Guard interval & $T_{g}$ & $10.0 \mathrm{~ms}$ \\
\hline Number of Guard Samples & $N_{g}$ & 60 \\
\hline Symbol Constellation & & $\mathrm{BPSK}$ \\
\hline Blocks Per Packet & $N_{b l k}$ & 4 \\
\hline Blanking Period Between Packets & & $3 \mathrm{sec}$ \\
\hline \multicolumn{2}{|c|}{ LFM Interference Parameters } & Value \\
\hline Center Frequency & $f_{c, I}$ & $11 \mathrm{kHz}$ \\
\hline Sweep Direction & & $\mathrm{Up}$ \\
\hline Bandwidth & $B_{I}$ & $B / 4, B / 2,3 B / 4$ \\
\hline Duration & $T_{I}$ & $T / 8, T / 4, T / 2$ \\
\hline Repetition Rate & $R_{I}$ & $1.25 T=213.3 \mathrm{~ms}$ \\
\hline Start Time of First Transmission & $T_{s t a r t}$ & 8.1071 \\
\hline
\end{tabular}

Table 8: AUTEC December 2014 OFDM and LFM transmit parameters. 


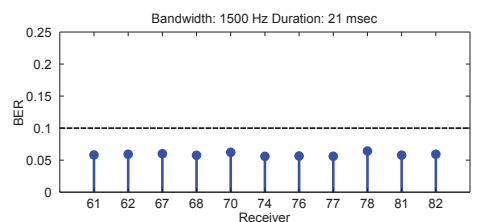

(a)

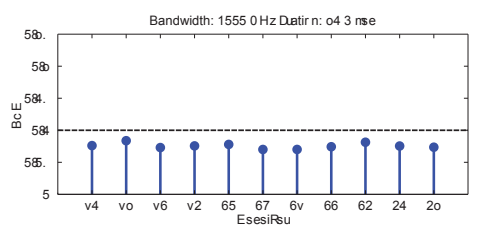

(d)

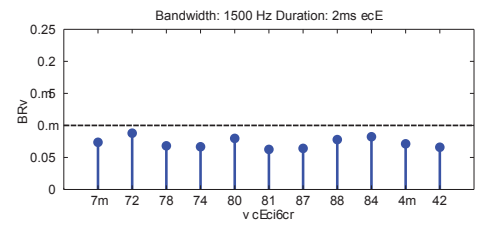

(g)

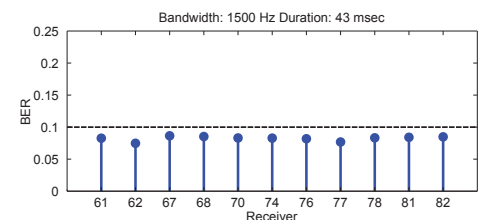

(b)

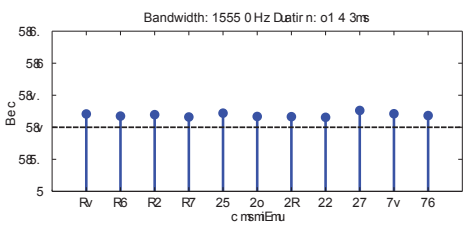

(e)

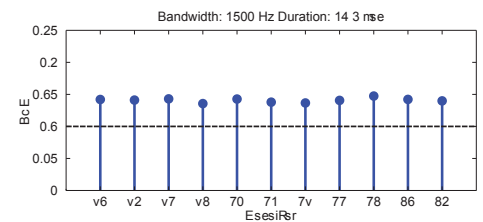

(h)

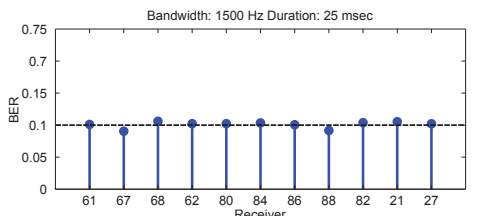

(c)

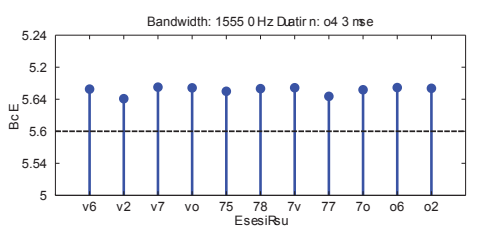

(f)

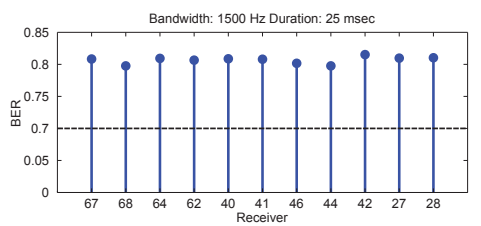

(i)

Figure 36: Uncoded bit error rate on simulated channel with no interference mitigation.

The BERs shown here are due to interference only. The effects of the underwater channel and additive noise (sea noise) are not included. Thus, the BER observed during the experiment will likely be higher.

generate received blocks with BERs greater than 0.10 because sea noise and the effects of the underwater acoustic channel will drive the BER higher.

Fig. 37 indicates the level of interference received on three receivers $(68,70$, 82) by time domain sample number for the different transmitted blocks. Receiver 68 is approximately equidistant from the transmitters; receiver 70 is closer to the interference transmitter; and receiver 82 is closer to the OFDM transmitter. The color scale is in decibels, with warm colors indicating higher levels of interference and cool colors less interference. Black indicates samples suffering virtually no interference. The time duration of the interference increases across the columns of the figure. The figure shows that even for a single receiver, different time domain portions of the received block will suffer interference. Since each block carries the 
same message, recorded data from a single receiver should satisfy the requirement to characterize performance as a function of the time orthogonality of the interference (EO2.2). This is an improvement from the AUTEC-0514 experiment where for a given interfering signal on a given receiver, the interference corrupted the same portions of the received signal as can be seen in the received signals shown in Fig. 34.

\subsection{Data Collected}

A quick look at the data collected during AUTEC-1214 shows that the experimental design improved upon the AUTEC-0514 experiment. Fig. 38 shows data packet receptions on receivers $67,68,74,76,77$ and 81 from the AUTEC-1214 experiment. The vertical dashed black lines delineate the OFDM blocks. As expected, the LFM interference corrupts different portions of each block. This is an improvement over the AUTEC-0514 experiment where the interference corrupted the same portion of the OFDM block on each receiver because it was transmitted synchronously with the OFDM data packets. Furthermore, the noise level and interference level vary among the receivers confirming that transmitting all the data at full power was a viable approach to generating a data set with varying SNR and SIR due to the spatial extent of the network. Unlike the AUTEC-0514 experiment, the direct path reception was not the strongest multipath reception. As shown in Fig. 39, the surface bounce path carried the strongest received signal. The surface bounce channel observed during the AUTEC-1214 experiment is considerable longer than the direct path channel observed during AUTEC-0514 as can be seen in Fig. 40 which shows OFDM blocks received on hydrophone 76 during AUTEC-0514 (panel a) and AUTEC-1214 (panel b). These receptions are representative samples of the received block from each experiment. The vertical dashed

black lines delineate the start and stop times of the OFDM block after completing 


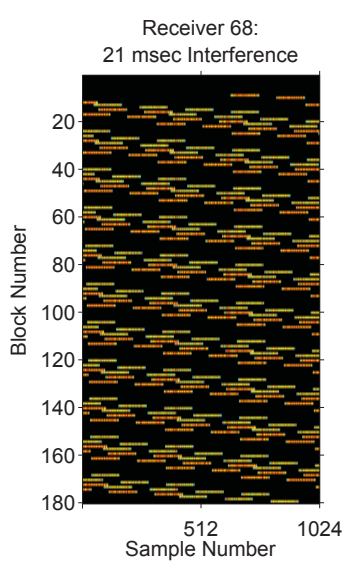

(a)

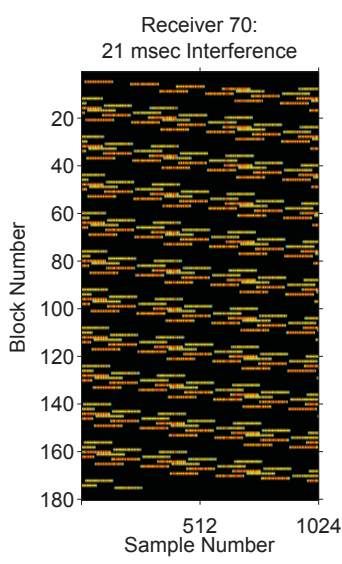

(d)

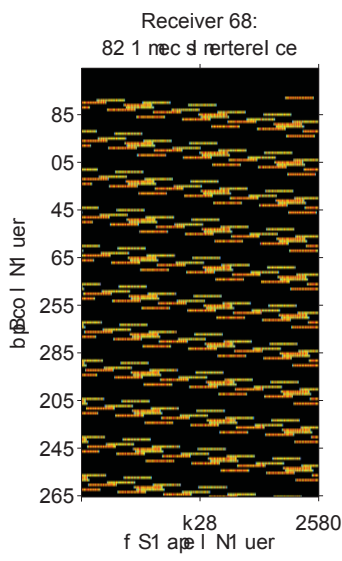

(g)

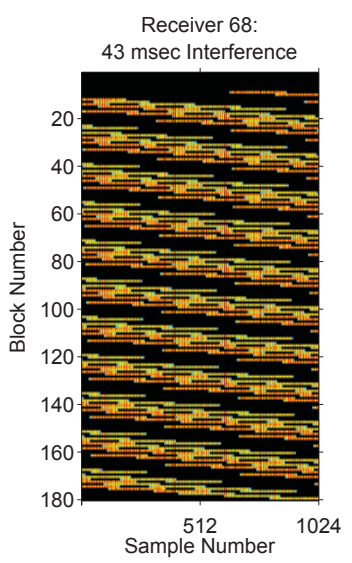

(b)

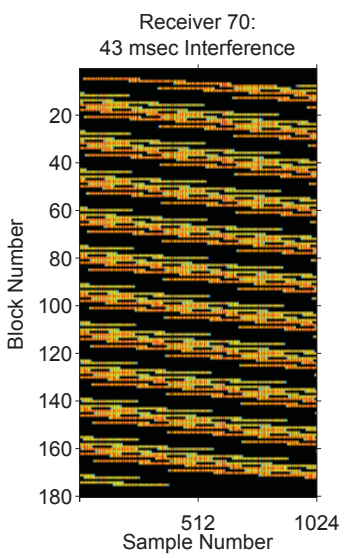

(e)

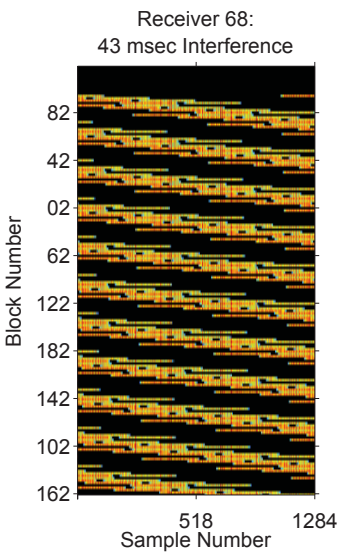

(h)

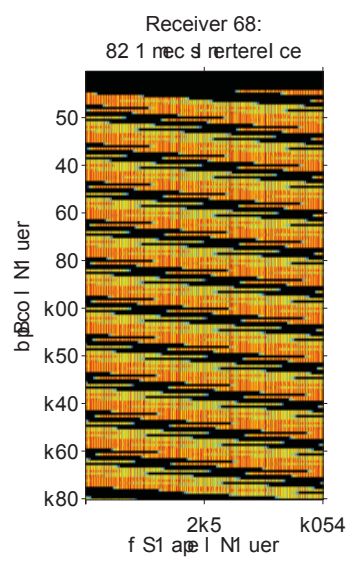

(c)

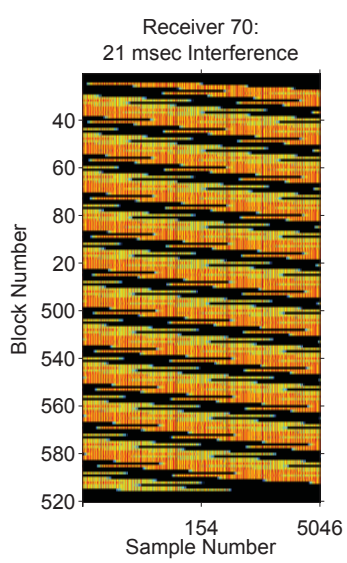

(f)

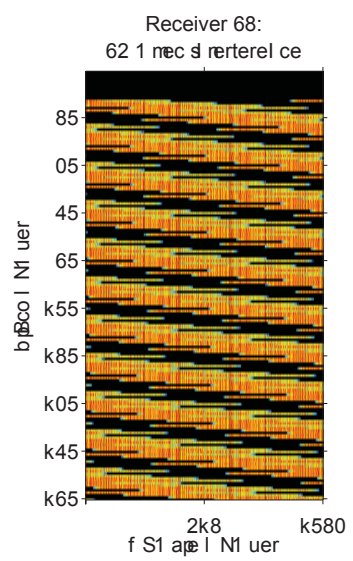

(i)

Figure 37: Presence of interference on various receivers.

Note that black indicates samples suffering virtually no interference $(\mathrm{SIR} \geq 10 \mathrm{~dB})$. 
time synchronization. Ten milliseconds of time (equivalent guard interval used in AUTEC-1214) is shown before and after each block. As discussed above and clearly shown in the figure, the direct path channel observed in AUTEC-0514 was much shorter than ten milliseconds. Unfortunately, the surface bounce channel observed in AUTEC-1214 is longer than the guard interval as clearly evidenced by the signal from the previous block bleeding over into the guard interval and interfering with the selected block. The reception of the selected block also extends to occupy the entire guard interval and part of the next block creating inter-block interference. The longer channel and the inter-block interference will complicate channel equalization. Processing the data from AUTEC-1214 is left for future work. Planning for the next experiment should anticipate that the direct path signal may not be the strongest and allow for a longer channel impulse response to avoid inter-block interference.

\subsection{Channel Model}

This section describes the channel model developed to aid in the design of the AUTEC-1214 experiment. The development of a channel model facilitated the development of the transmit waveforms and transmit schedule to satisfy the test objectives. Specifically, modeling aided the selection of the OFDM symbol duration, guard interval, number of blocks per packet, the blanking period between packets, the LFM interference duration, bandwidth and repetition rate.

The Sonar Simulation Toolkit (SST) [97] and the Acoustic Channel Simulator [98] were considered as modeling tools for developing the transmit waveforms for the December test. Both models provide sophisticated, state-of-the-art simulations of the underwater acoustic channel. However, there is a considerable learning curve associated with each and since the purpose of employing the models was to

design an experiment and work with the real data obtained from the experiment 

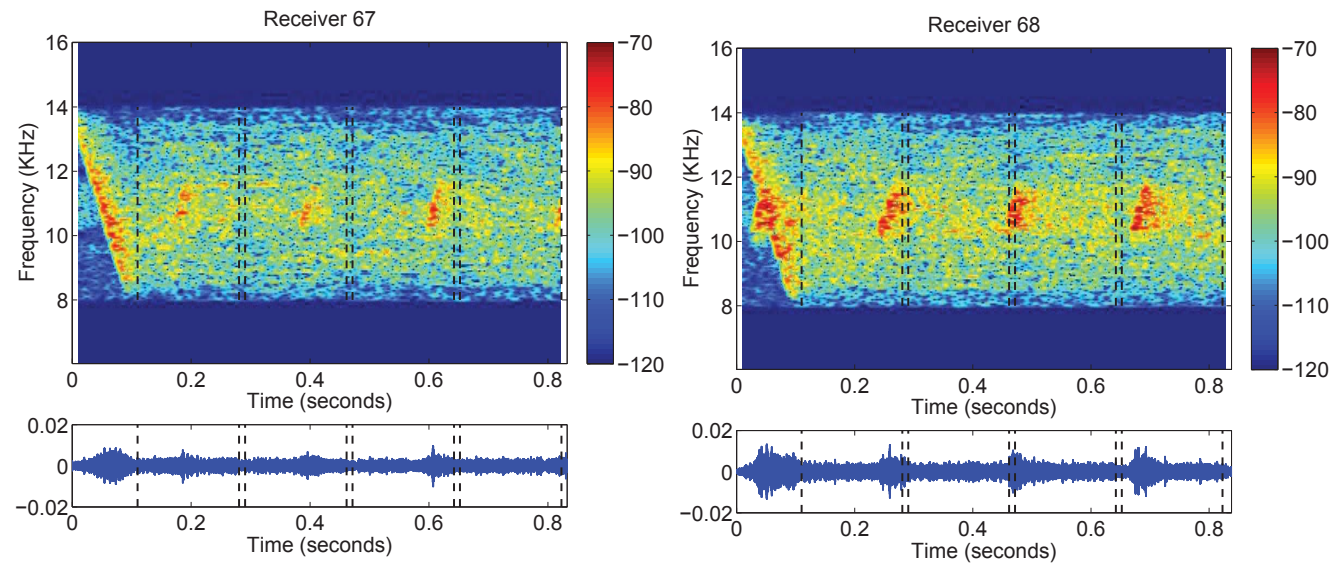

(a)

(b)

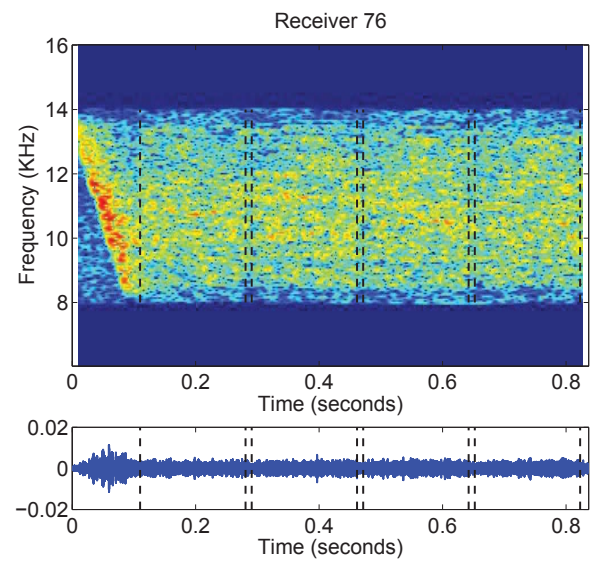

(c)

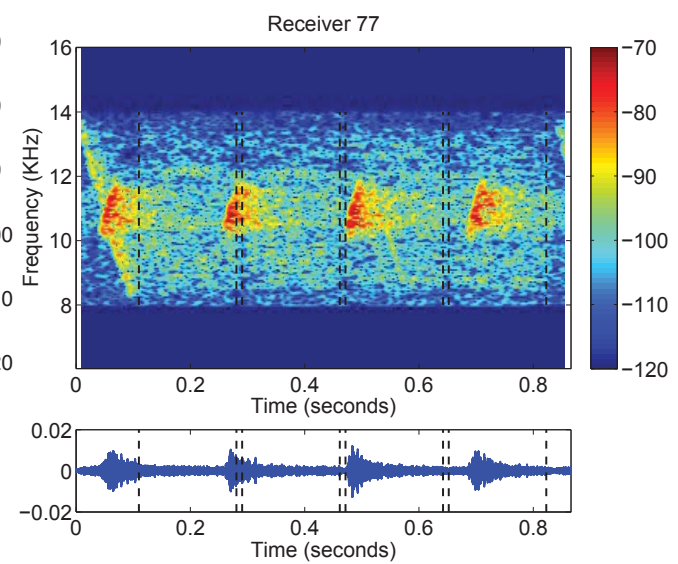

(d)

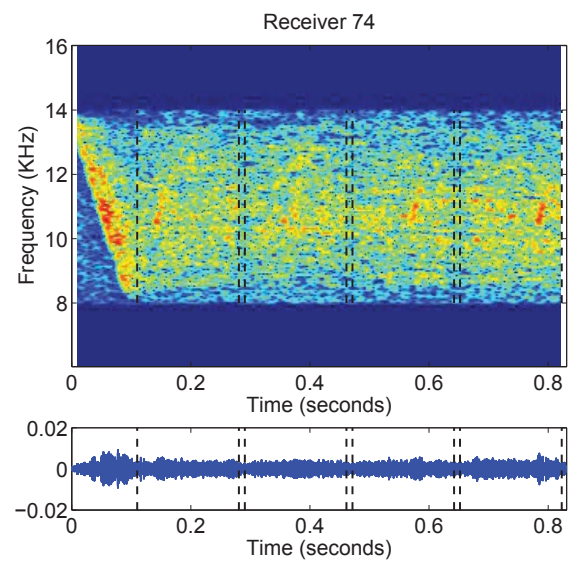

(e)
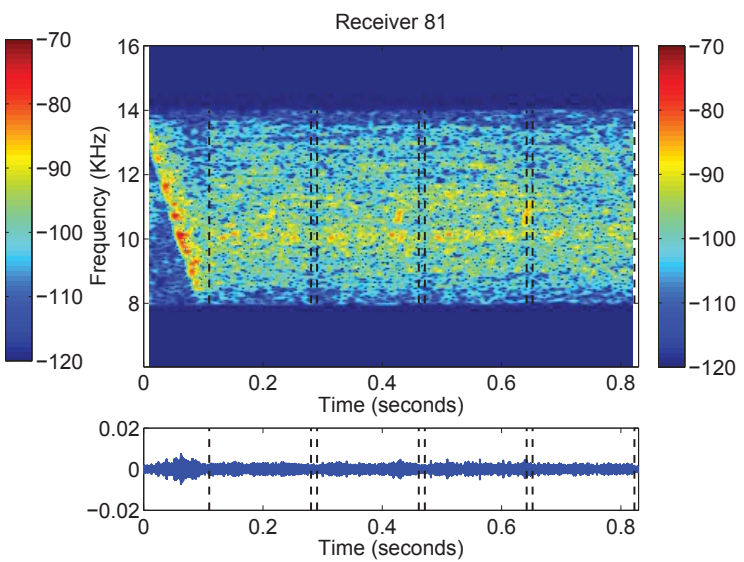

(f)

Figure 38: Received data packets from various receivers during AUTEC-1214. The black lines delineate the OFDM blocks. As expected, the LFM interference corrupts different portions of each block and the level of the interference is different on each receiver. 


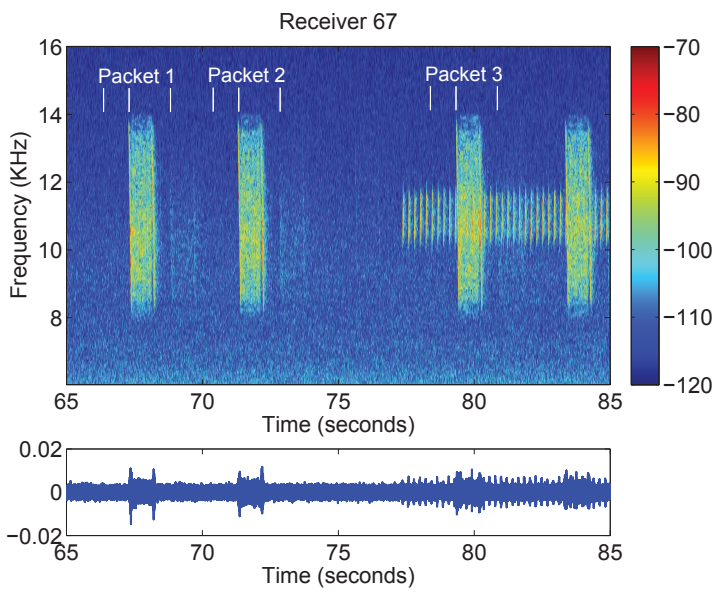

(a)

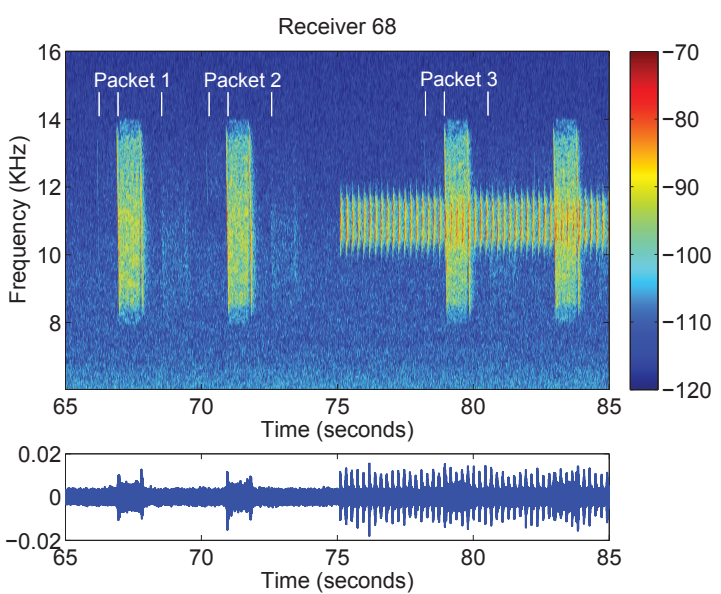

(b)

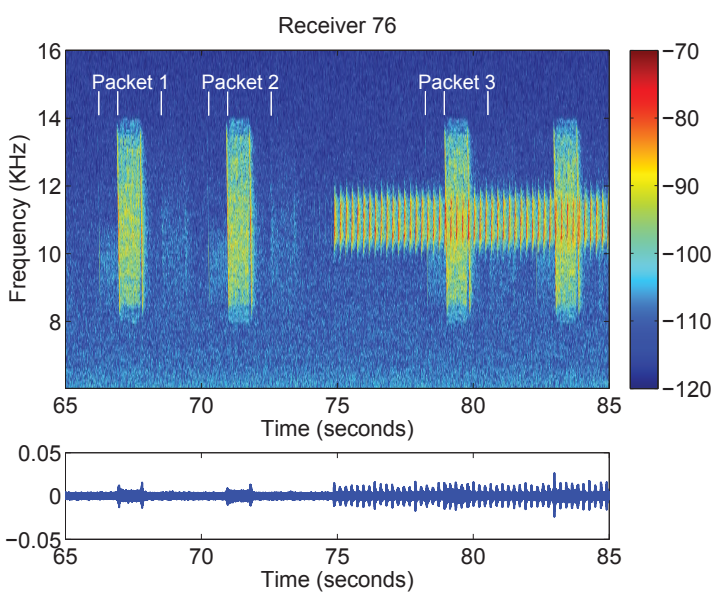

(c)

Figure 39: Receptions of data packets 1-4 on various receivers from AUTEC-1214. Spectrograms showing the first four received data packets on Receivers 67, 68 and 76. The white lines indicate the time of the multipath arrivals. Note that the surface bounce arrival is the strongest in contrast to AUTEC-0514 where the direct path arrival was the strongest. 


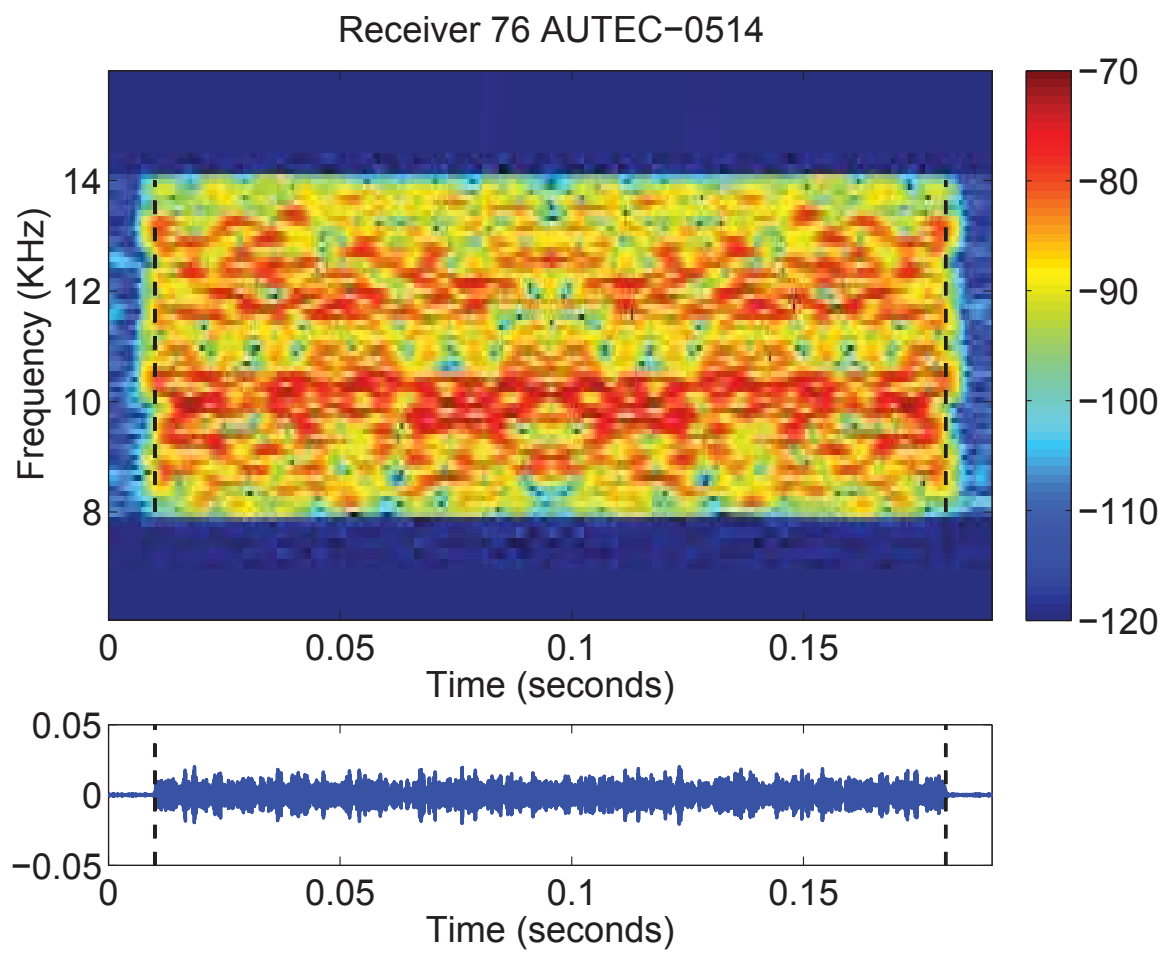

(a)

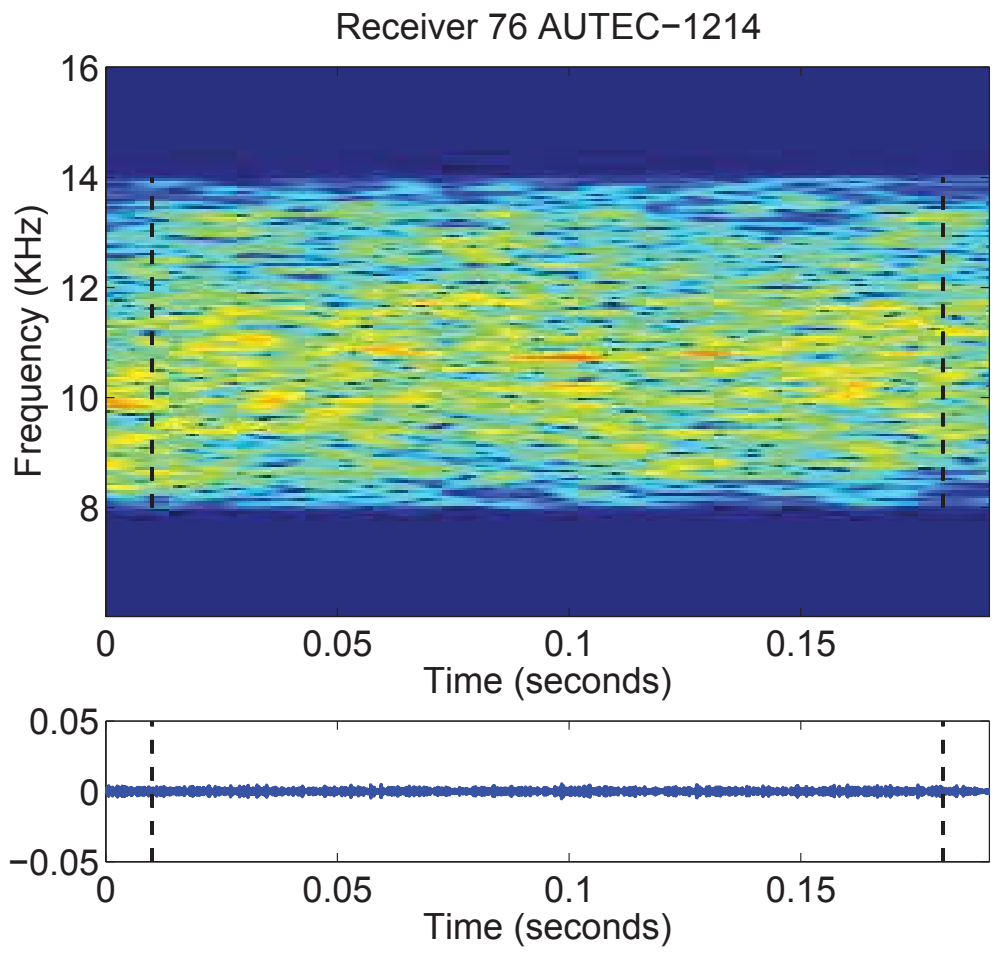

(b)

Figure 40: Reception of a data block on receiver 76 from AUTEC-0514 (panel a) and AUTEC-1214 (panel b). 
rather than simulated data, the option of developing a very simple channel model leveraging data collected during the AUTEC-0514 was pursued instead.

Analysis of the AUTEC-0514 data showed that the interaction of the direct path and first surface bounce reception had the largest effect on the received signal. Thus, the key concern from the perspective of experimental design is obtaining a reasonable estimate of the time of the direct path and surface bounce arrivals from the two transmitters to the various receivers. There is some variability among the sound velocity profile from AUTEC-0514 and the historical sound velocity profiles reviewed from November 2014 and December 2013 as shown in Fig. 41. However, the variation is not so great as to invalidate the modeling approach since only a rough estimate of the channel delays is required. Note that the SVP observed during AUTEC-1214 is quite similar to the SVP from the AUTEC-0514 experiment. Consequently, a simple two tap channel model was developed from each transmitter to the receivers with the amplitude of the direct path and surface bounce taps fixed at 1 and 0.5 respectively for all receivers. The channel delays were determined with the aid of AUTEC-0514 data. The resulting channel model is shown in Fig. 42 where dark blue indicates the direct path tap from the communication signal transmitter to receiver; light blue indicates the surface bounce tap from the signal transmitter to receiver; red indicates the direct path tap from the interference transmitter to receiver; magenta indicates the surface bounce tap from the interference transmitter; black indicates the mean surface bounce arrival time and amplitude observed in AUTEC-0514.

Histograms of the received time difference of arrival of the direct path and surface bounce receptions show that the channel delays were very stable (varying less than $25 \mathrm{~ms}$ on each observed channel) over the course of AUTEC-0514 as shown in Fig. 43. The observations from AUTEC-0514 shown in Fig. 44 indicate that the 


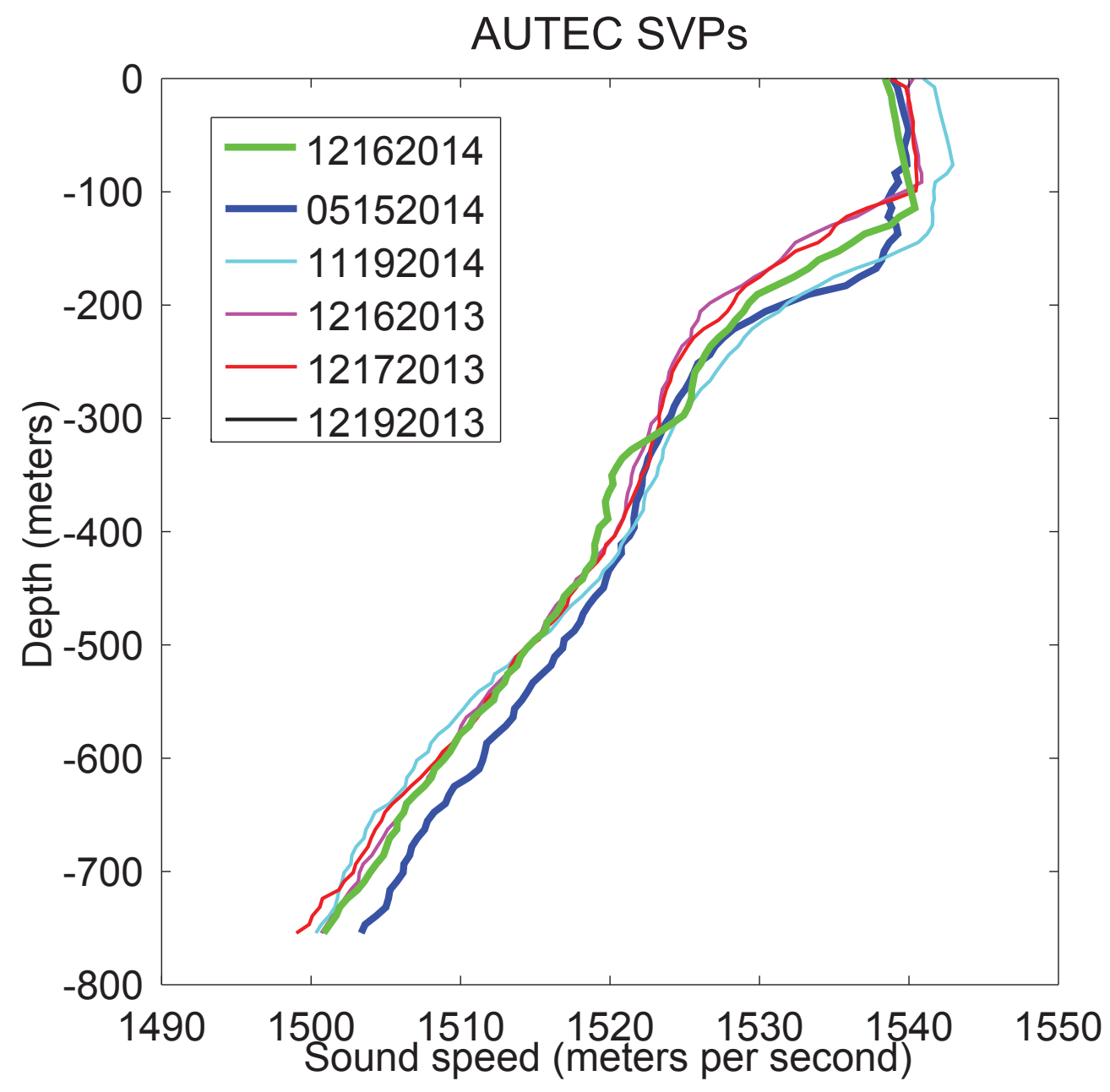

Figure 41: AUTEC-0514, AUTEC-1214 and other selected sound velocity profiles from AUTEC. 


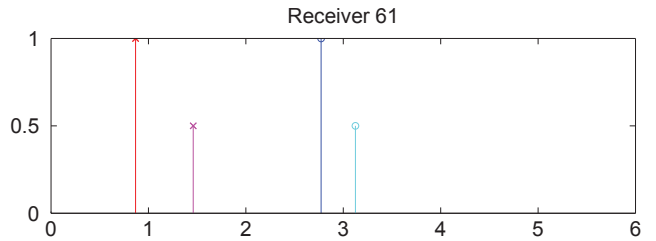

(a)

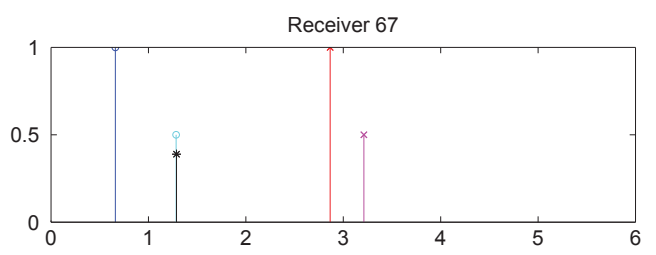

(c)

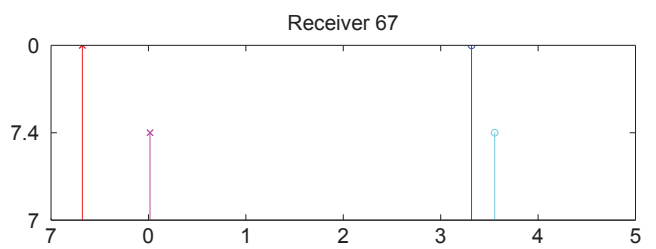

(e)

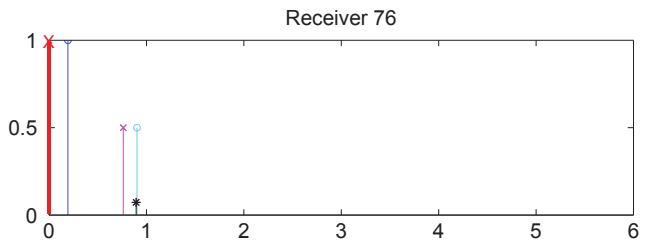

(g)

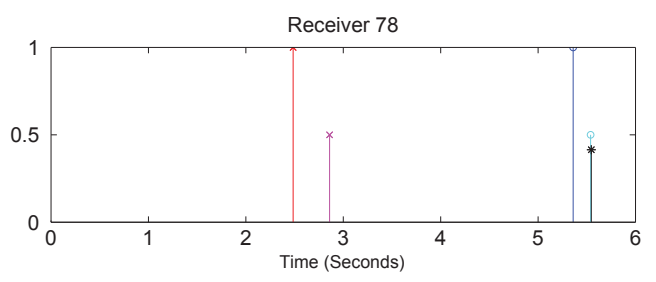

(i)

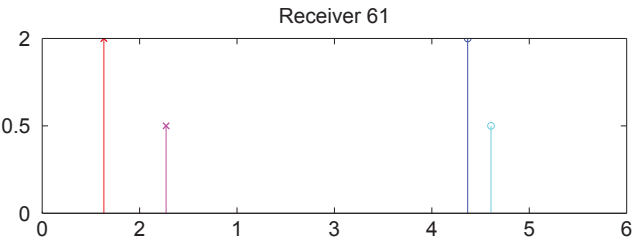

(b)

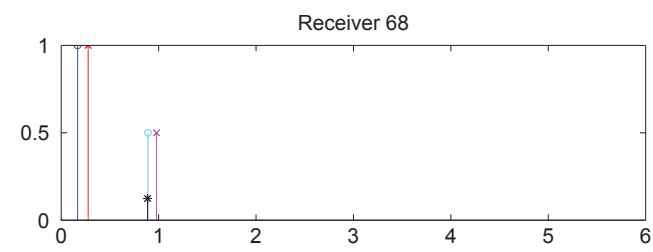

(d)

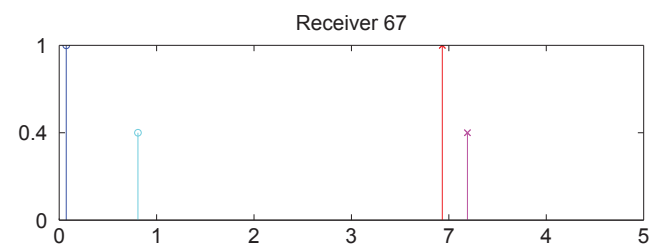

(f)

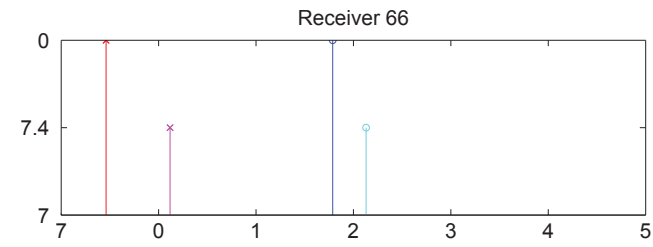

(h)

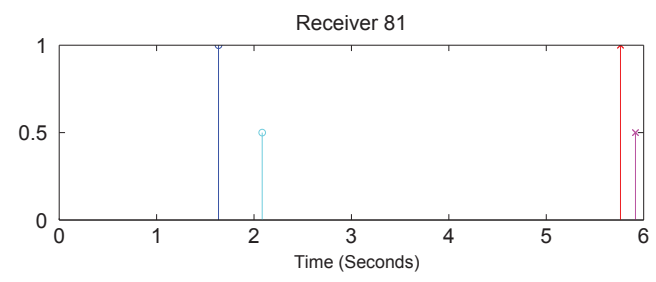

(j)

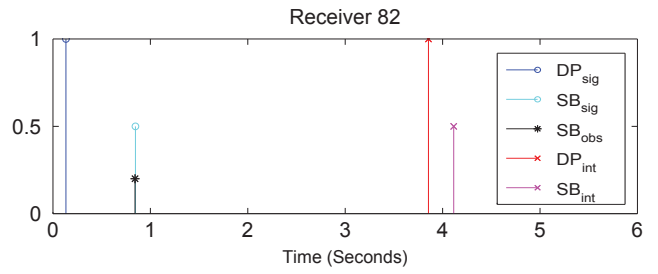

(k)

Figure 42: Channel models from nodes 75 and 69 to various receivers. 


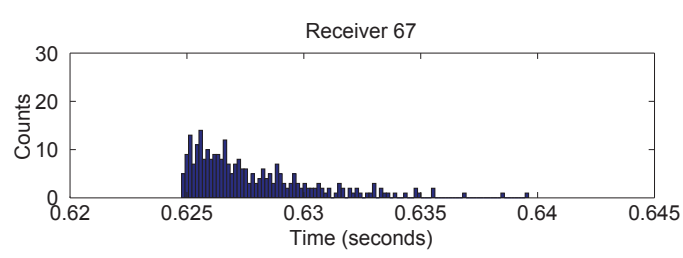

(a)

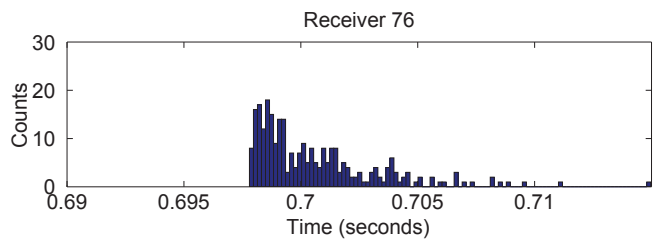

(c)

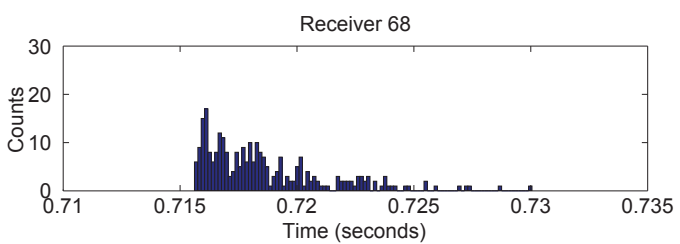

(b)

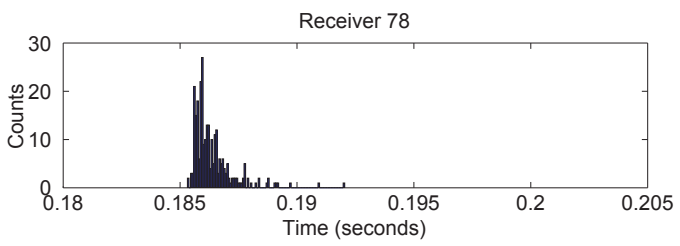

(d)

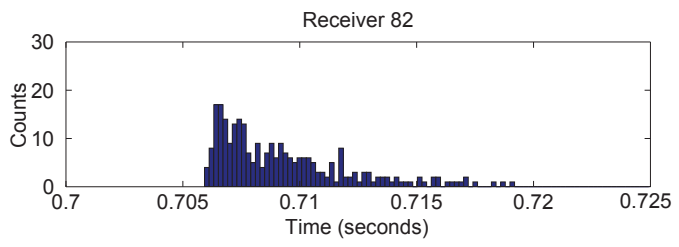

(e)

Figure 43: Histograms of the difference in arrival time of direct path and surface bounce receptions of the channel probe.

amplitude difference between the direct path and surface bounce receptions is variable. No attempt was made to account for this variability since the purpose of the channel model was to accurately capture the channel delays. Note that the left and right columns of Fig. 44 plot the peak matched filter output of the channel probe direct path and surface bounce arrivals respectively. The same scaling is used for all the plots in the right column. In the left column, amplitude scale range is 15 units with the exception of panel (c) and (e) which are 25 and 45 units respectively.

\subsubsection{Physical Basis for Channel Model}

The observation from AUTEC-0514 that the important ray paths are either direct ray (e.g. refracted rays) or surface bounce rays (e.g. refracted surface reflected) is characteristic of deep ocean propagation. The geometry is shown in 


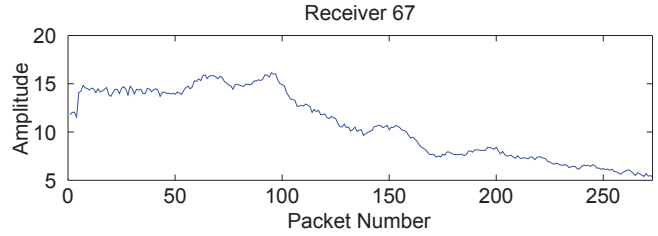

(a)

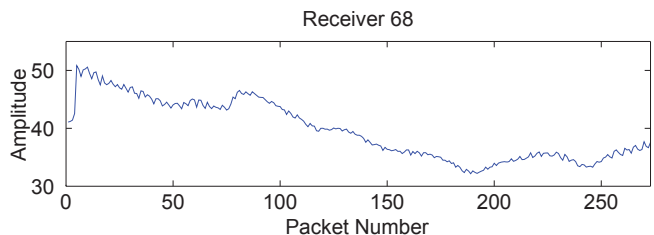

(c)

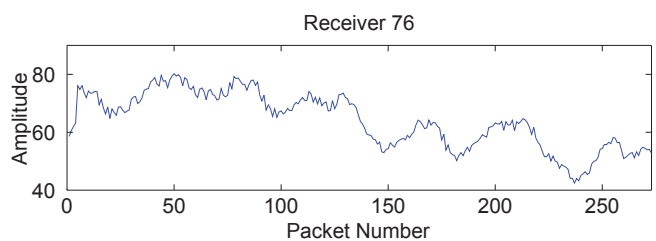

(e)

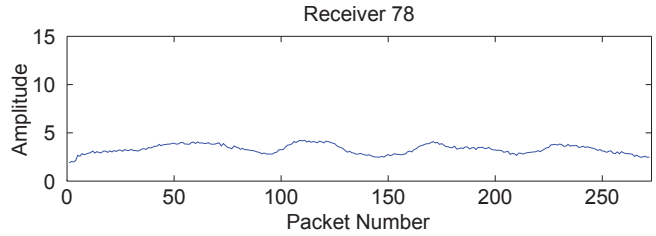

(g)

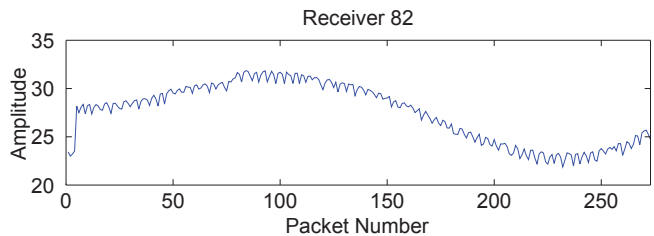

(i)

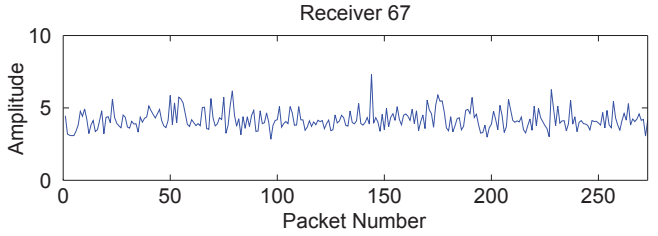

(b)

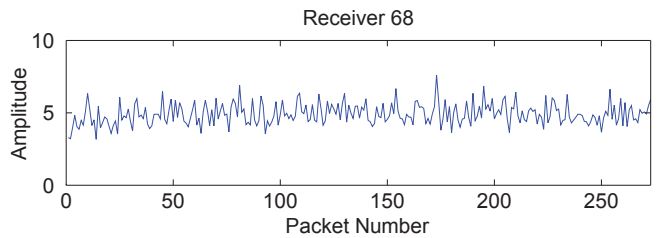

(d)

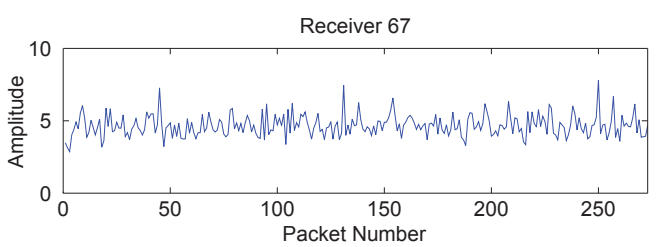

(f)

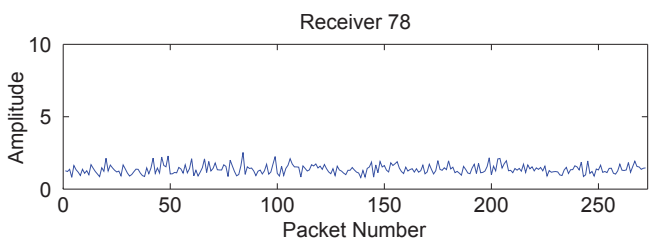

(h)

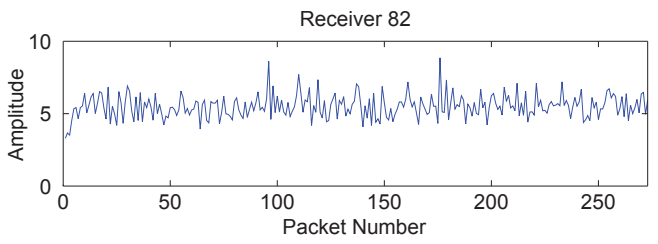

(j)

Figure 44: Peak matched filter output for direct path and surface bounce receptions of the channel probe. 


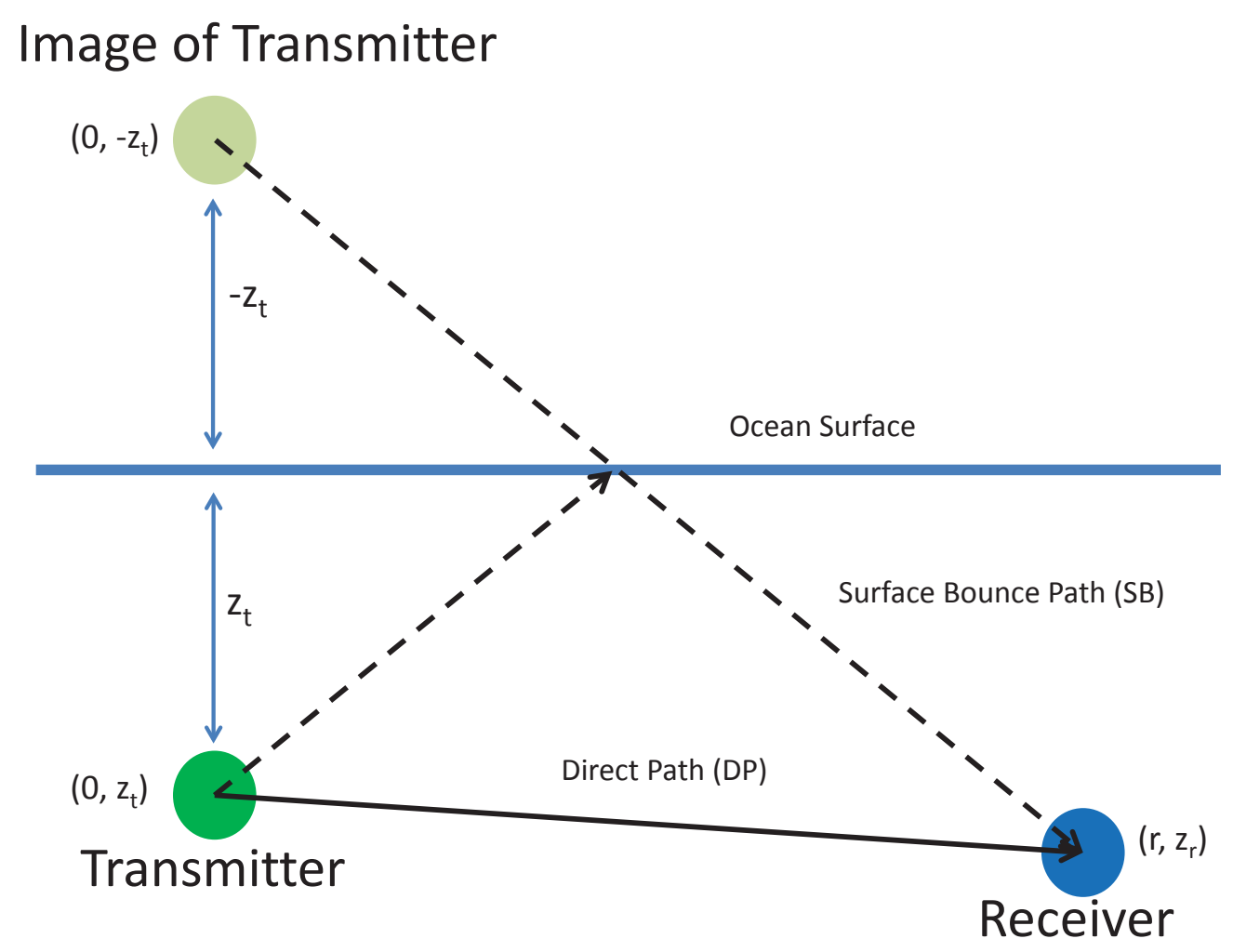

Figure 45: Direct path and surface bounce rays propagating from transmitter to receiver.

Fig. 45 with the transmitting source at position depth $z_{t}$ below the surface $(z=0)$. There are two energy paths connecting the transmitter and receiver: the direct path (DP) and the surface bounce path (SB). Assuming specular reflection at the sea surface, the reflected path appears to originate from the image of the transmitter at $\left(0,-z_{t}\right)[23]$. The analytic solution may be found by either the image method discussed in section 1.4.2 or by applying Green's theorem to the boundary problem of a point source in a fluid half-space in section 2.3.4 of [23]. For the purposes of designing the experiment, we mention the above to demonstrate that there is a physical basis for the selected model. The model assumed an effective direct path sound speed $c_{D P}$ and a separate surface bounce sound speed $c_{S B}$. The time of arrival for the direct and surface bounce paths from a transmitter at position 
$\left(x_{t}, y_{t}, z_{t}\right)$ to a receiver at position $\left(x_{r}, y_{r}, z_{r}\right)$ is given by:

$$
\begin{aligned}
& T O A_{D P}=\sqrt{\left(x_{r}-x_{t}\right)^{2}+\left(y_{r}-y_{t}\right)^{2}+\left(z_{r}-z_{t}\right)^{2}} / c_{D P} \\
& T O A_{S B}=\sqrt{\left(x_{r}-x_{t}\right)^{2}+\left(y_{r}-y_{t}\right)^{2}+\left(z_{r}+z_{t}\right)^{2}} / c_{S B}
\end{aligned}
$$

respectively, and the time difference of arrival of the direct path and surface bounce arrivals is simply:

$$
T O D A=T O A_{D P}-T O A_{S B}
$$

Based on the direct path and surface bounce TDOA on receivers $67,68,76,78$ and 82 from AUTEC-0514, an effective surface bounce sound speed and direct path sound speed of 1510 and 1490 meters per second was determined. Applying these sound speeds to Eqs. (113) and (114) determined the delays for the channel model shown in Fig. 42 where all delays are referenced to the earliest direct path arrival, which occurs on receiver 76 .

\subsection{Summary}

This chapter described the planning for the for the AUTEC-1214 experiment. The goal of the AUTEC-1214 experiment is to assess the performance of the SDR and PIC algorithms in mitigating the effects of SAI on communication signals in an undersea environment. A set of experimental objectives and data collection requirements support this goal. The experimental procedure involves simultaneously transmitting communication and interfering signals and recording the received waveforms. A simple channel model developed from the data collected during AUTEC-0514 facilitated the development of the transmit waveforms and transmit schedule. The resulting plan improves upon the AUTEC-0514 plan in the following respects:

- The range of interference durations and bandwidths is likely to result in an uncoded BER of greater than 0.1. In the AUTEC-0514 experiment, the in- 
terference bandwidths and time durations resulted in BERs that were too low to effectively test the algorithms directly on the received data. Consequently, "pseudo-experimental" data had to be constructed by amplifying the received interference and overlaying it on top of the received communication signals.

- The interference occurs in different time slots within the OFDM packets received each channel. This means that more OFDM blocks should be available for testing the algorithms in contrast to the AUTEC-0514 experiment where the transmission of the communication signal and interference was synchronized such that the interference always corrupted the same portion of the received communication signal on each receiver.

- Unlike AUTEC-0514, the message bits and parity check bits are interleaved across the data subcarriers. Therefore, the effects of received interference will be distributed across the structure of the code.

A quick look at the received data from AUTEC-1214 shows that the interference did occur in different time slots within the OFDM packets on each channel. Unfortunately, the strongest received signal occurred via a path with a longer impulse response than the guard interval resulting in all the receptions being contaminated with inter-block interference. This will complicate processing the received data, which is left to future work. 


\section{CHAPTER 5}

\section{Conclusions and Future Work}

\subsection{Conclusions}

Many acoustic channels suffer from interference which is neither narrowband nor impulsive. This relatively long duration partial band interference can be particularly detrimental to system performance. In operational networks, many dropped messages are lost due to partial band interference which corrupts different portions of the received signal depending on the relative position of the interferers, information source and receivers due to the slow speed of propagation. A survey of recent work in interference mitigation and orthogonal frequency division multiplexing (OFDM) as well as observations from the Atlantic Undersea Test and Evaluation Center (AUTEC) provided motivation to develop a spatial diversity receiver for use in underwater networks.

The application of this work extends beyond the AUTEC network to cabled acoustic networks in general and other situations where interference corrupts receptions on widely separated receivers. As we continue to explore and operate in the oceans, the number of activities grows. Many of these activities generate signals of interest to the user of the application at hand, but create interference from the perspective of other users. Communications is an essential aspect of many of these operations. For example, autonomous undersea vehicles require a reliable communications link to send and receive data to remote users. As the undersea environment becomes more acoustically congested, understanding the limits interference places on performance and developing approaches to mitigate its effects are important areas of research. This research examined approaches to leverage the spatial diversity of underwater acoustic communications networks suffering from interference. 
The concept of combining multiple receptions of the same transmitted signal is not new. Information is available for combining at multiple different levels: 1) the received waveform, 2) the detected symbols and 3) the decoded information. In general, a diversity processor could operate on any of these levels or among the different levels. This work focused on combining the received waveforms. The concept of combining received waveforms has been a standard approach for decades. Maximum ratio combining (see chapter 7 , section 5 of [33]) weights the received waveforms based on the signal-to-noise ratio at each receiver. Optimal ratio combining (ORC) [92] also takes advantage of the differential fading received signals experience, but additionally, considers the impact of co-channel interferers in calculating the weighting of the received waveforms. ORC was specifically developed to combat co-channel interference on Rayleigh fading channels for mobile radio. Whereas ORC takes advantage of the differential fading of the virtually synchronously received interference among the receivers, the algorithm developed here relies on the slow speed of signal propagation underwater which results in the received interference exhibiting some degree of time orthogonality. Underwater receptions also experience differential fading and when the interference is not time orthogonal, the algorithm makes use of this in a manner similar to ORC.

The spatial diversity receiver for underwater communications identifies portions of the signal suffering from interference on different receivers, removes these portions of the signal and then optimally combines the remaining clean portions of the signal. Analytic results of performance for receivers on additive white Gaussian noise channels suffering time orthogonal interference demonstrated the effectiveness of the spatial diversity combining strategy as compared to conventional maximum ratio combining. Simulation results on time invariant channels confirmed the effectiveness of the algorithm under more complex channel conditions. 
Comparison of the spatial diversity receiver performance to the recently developed single receiver parameterized interference cancellation algorithm was made using results from an experiment conducted at the AUTEC network which consists of multiple distributed cabled hydrophones that receive data transmitted over a timevarying multipath channel in the presence of partial band interference produced by interfering active sonar signals.

The spatial diversity receiver algorithm and the parameterized interference cancellation algorithm address the problem of interference in fundamentally different ways: the spatial diversity receiver blanks the interference, while the parameterized cancellation algorithm coherently removes it from the desired signal. Both algorithms require a priori knowledge to aid in frequency domain interference detection. SDR uses a priori knowledge to set the size of the time domain blanking window, whereas in PIC, a priori knowledge determines the number of coefficients used to estimate the interference. In SDR imprecise a priori knowledge leads to performance degradation because improperly sizing the blanking window results in interference contaminating the signal if the window is too small or loss in averaging gain if the window is too large. In contrast, for PIC, if the number of coefficients to estimate is too large, the excess coefficients get little weight. Furthermore for PIC, the important parameter in determining the number of coefficients to estimate is the time bandwidth product. Interfering signals with different time durations and bandwidths but a time bandwidth product less than the selected value will still be estimated accurately and subtracted. SDR requires the time duration and bandwidth to be known separately and its performance is best when applied to signals with the specified time duration and bandwidth. While SDR requires more detailed prior knowledge than PIC, its performance is less dependent on the SIR. Analytic expressions for the performance of SDR are available under certain condi- 
tions and the loss in performance due to mismatching the size of the time domain blanking window can be determined. PIC is a more complicated algorithm and analytic characterizations of performance are not available. Finally, SDR requires operations in both the time and frequency domains while PIC operates exclusively in the frequency domain.

Both techniques are effective mitigation strategies with SDR being most effective and realizing the most gain at low SIRs while PIC is effective at moderate SIRs. The two approaches are complimentary in this sense: SDR is not limited by low SIR, but it requires multiple receivers; PIC is limited at low SIR but operates on a single receiver. In an undersea network one effective multi-channel receiver strategy would be to employ PIC on receptions suffering from moderate interference and transition to the multi-channel SDR technique when PIC fails. Alternatively, the SDR strategy could be employed as the default and PIC could be attempted in cases where the lack of time orthogonality and severity of the interference make blanking or averaging ineffective operations. In these cases coherent cancellation could be attempted.

\subsection{Future Work}

The development of a receiver that effectively uses the SDR algorithm under extremely high interference conditions and the PIC algorithm when the interference is less severe is a logical next step. The approach for combining multiple receivers using the PIC algorithm could also be explored. That is, how is the combining done most effectively? At the waveform level after the interference has been subtracted? By sharing information about satisfied parity checks so that additional tones can be used for equalization among independently operating equalizers? Is the decoding done independently on each receiver and then the information shared at the output or are the symbols derived from a weighted combination of the re- 


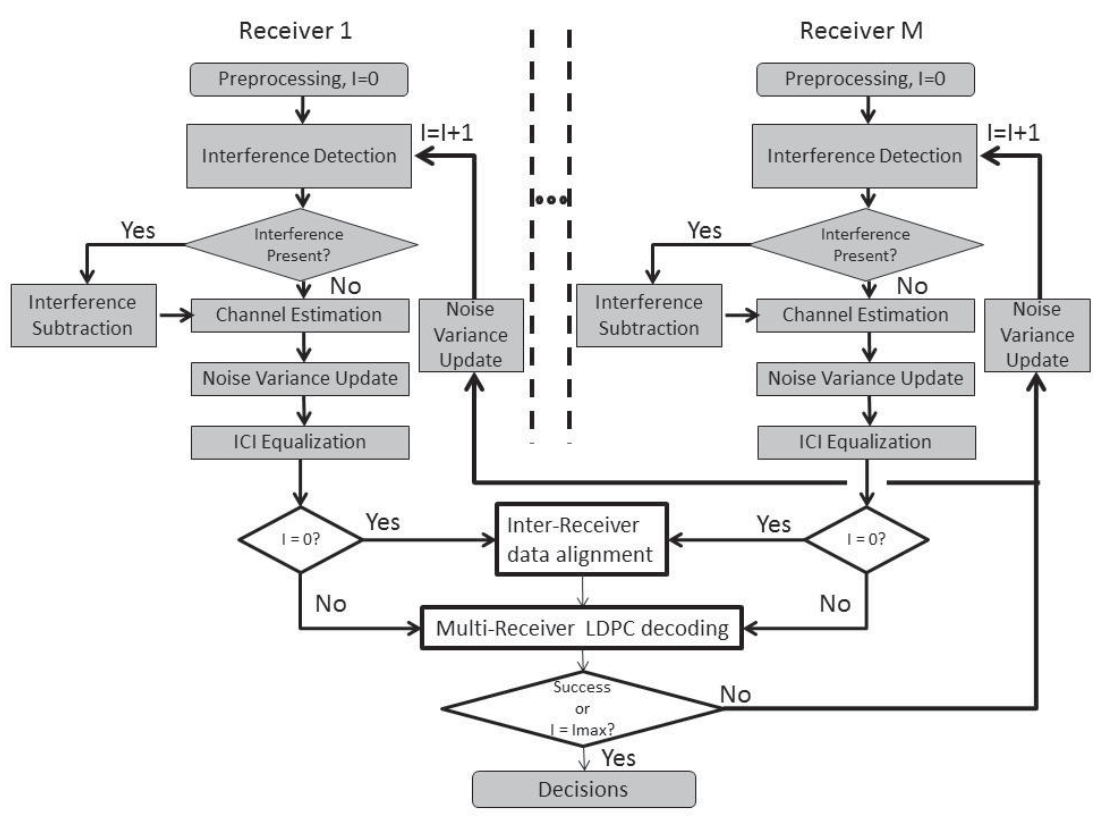

Figure 46: Spatial diversity receiver.

ceived waveforms supplied to a single decoder? Fig. 46 shows one example of how the PIC algorithm might be extended to multiple receivers. The white boxes indicate added functionality: inter-receiver data alignment and multi-receiver LDPC decoding.

The problem of selecting the correct waveforms to combine needs to be addressed. A practical receiver must ensure that the source of the combined waveforms or extracted data is the same. Study of transmit schemes that facilitate information combining in single and multi-user environments could be undertaken as part of this work.

For SDR, the following modifications to the current implementation would likely result in performance gains:

- Replacing the least squares equalizer with a weighted least squares equalizer. In this work, no attempt was made to mitigate detected interference before equalization. Performance improvement especially at low SIR and low SNR would likely be realizable if the equalization process accounted for the 
presence of interference.

- Development of an adaptive SDR detector based the analytic performance equations.

- Extension of the algorithm to handle multiple different interfering signals of different time durations and bandwidths.

From a theoretical perspective, a next logical step for SDR is to derive an analytic expression for an additive white Gaussian noise channel without the requirement that the interference be time orthogonal. 
We shall not cease from exploration

And the end of all our exploring

Will be to arrive where we started

And know the place for the first time.

Through the unknown, remembered gate

When the last of earth left to discover

Is that which was the beginning;

At the source of the longest river

The voice of the hidden waterfall

And the children in the apple-tree

Not known, because not looked for

But heard, half-heard, in the stillness

Between two waves of the sea.

Quick now, here, now, always-

A condition of complete simplicity

(Costing not less than everything)

And all shall be well and

All manner of thing shall be well

When the tongues of flame are in-folded

Into the crowned knot of fire

And the fire and the rose are one. ${ }^{1}$

\footnotetext{
${ }^{1}$ Conclusion of "Little Gidding" by T. S. Eliot
} 


\section{LIST OF REFERENCES}

[1] A. Baggeroer, "Acoustic telemetry - an overview," IEEE Journal Oceanic Eng., vol. 9, no. 4, pp. 229-235, 1984.

[2] M. Stojanovic, "Recent advances in high-speed underwater acoustic communications," IEEE J. Oceanic Eng., vol. 21, no. 2, pp. 125-126, 1996.

[3] R. J. Vaccaro, "The past, present and future of underwater acoustic signal processing," IEEE Signal Processing Magazine, vol. 15, no. 4, pp. 21-51, 1998.

[4] J. C. Presig and D. Brady, "Underwater acoustic communications," in Wireless Communications. New Jersey: Prentice Hall, 1998.

[5] D. B. Kilfoyle and A. B. Baggeroer, "The state of the art in underwater acoustic telemetry," IEEE Journal of Oceanic Engineering, vol. 25, no. 1, pp. $4-27,2000$.

[6] M. Stojanovic, "Underwater acoustic communication," in Encyclopedia of Electrical and Electronics Engineering. Wiley, 2000.

[7] J. Proakis, E. Soxer, J. Rice, and M. Stojanovic, "Shallow water acoustic networks," IEEE Communications Magazine, vol. 39, no. 11, pp. 114-119, 2001.

[8] L. Freitag, M. Stojanovic, D. Kilfoyle, and J. Presig, "A review of a decade of research and a perspective on future challenges," in Proc. of 7th Europan Conference on Underwater Acoustics, 2004.

[9] J. Partan, J. Kurose, and B. N. Levine, "A survey of practical issues in underwater networks," in Proc. ACM WUWNet, 2006.

[10] M. Chitre, S. Shahabudeen, L. Freitag, and M. Stojanovic, "Recent advances in underwater acoustic communications \& networking," in Proc. OCEANS 2008, vol. 2008-Supplement, no. 10.1109/OCEANS.2008.5289428, 2008, pp. $1-10$.

[11] W. Chen, "Physical layer design considerations for underwater acoustic sensor networks," in in Computer Science and Information Technology (ICCSIT), 3rd International Conference on, 2010.

[12] J. Heidermann, M. Stojanovic, and M. Zorzi, "Underwater sensor networks: Applications, advances, and challenges," Royal Society, 2012.

[13] "Submarine signaling," Scientific American Supplement, no. 2071, pp. 168170, September 1915. 
[14] D. Green, "Acoustic modems, navigation aids, and networks for undersea operations," in Proc. IEEE Oceans Conference, Marco Island, FL, Jan. 2010.

[15] I. Vasilescu, R. Kotay, D. Rus, M. Dunbabin, and P. Corke, "Data collection, storage, and retrieval with an underwater sensor network," in Proc. of the third ACM SenSys conference, no. 3, San Diego, June 2005, pp. 154-165.

[16] R. Hayford, D. Nagle, and J. Catipovic, "Undersea cellular network, Tongue of the Ocean Bahamas," in Proc. of the 4th ACM Workshop on Underwater Network (WuWNet), Berkerly, 2009.

[17] Z. Wang, S. Zhou, J. Catipovic, and P. Willett, "Parameterized cancellation of partial-band partial-block-duration interference for underwater acoustic OFDM," IEEE Transactions on Signal Processing, vol. 60, no. 4, pp. 1782$1795,2012$.

[18] "Grand bahama area code information," http://www.areacodehelp.com/ acsa/grand_bahama_area_code.shtml, accessed: 2015-02-22.

[19] J. A. Catipovic, "Performance limitations in underwater acoustic telemetry," IEEE Journal of Oceanic Engineering, vol. 15, no. 3, pp. 205-216, 1990.

[20] M. Stojanovic and J. Presig, "Underwater communication channels: Propagation models and statistical characterization," IEEE Communications Magazine, pp. 84-89, January 2009.

[21] J. Proakis and S. Masoud, Digital Communications, 5th ed. McGraw-Hill, 2008.

[22] K. Pelekanakis, "Harvesting time-frequency-space diversity with coded modulation for underwater acoustic communications," Ph.D. dissertation, Massachusetts Institute of Technology, 2009.

[23] F. B. Jensen, W. A. Kuperman, M. B. Porter, and H. Schmidt, Computational Ocean Acoustics. Springer, 2000, vol. 2nd Edition.

[24] W. S. Burdic, Underwater Acoustic Systems Analysis. Peninsula Publishing, 1990, vol. 2nd Edition.

[25] M. B. Porter, "A numerical method for computing ocean acoustic modes," Ph.D. dissertation, Northwestern University, 1984.

[26] M. B. Porter and E. L. Reiss, "A numerical method for ocean acoustic normal modes," J. Acoust. Soc. Am., vol. 76, pp. 244-252, 1984.

[27] M. B. Porter and E. L. Reiss, "A numerical method for bottom interacting ocean acoustic normal modes," J. Acoust. Soc. Am., vol. 77, pp. 1760-1767, 1985. 
[28] M. B. Porter and E. L. Reiss, "A numerical method for acoustic normal modes for shear flows," J. Sound and Vibration, vol. 100, pp. 91-105, 1985.

[29] W. A. Kuperman, M. B. Porter, J. S. Perkins, and R. B. Evans, "Rapid computation of acoustic fields in three-dimensional ocean environments," J. Acoust. Soc. Am., vol. 89, pp. 125-1133, 1991.

[30] J. S. Perkins, W. Kuperman, F. Ingenito, and J. Glattetre, "Modeling ambient noise in three-dimensional ocean environments," J. Acoust. Soc. Am., vol. 93, pp. 739-752, 1993.

[31] M. B. Porter and Y. C. Liu, "Finite-element ray tracing," Theoretical and Computational Acoustics, vol. 2, pp. 947-957, September 1994.

[32] B. Li, S. Zhou, M. Stojanovic, L. Freitag, and P. Willett, "Multicarrier communications over underwater acoustic channels with nonuniform doppler shifts," IEEE J. Ocean Eng, vol. 33, no. 2, 2008.

[33] A. Goldsmith, Wireless Communications. Cambridge Univsersity Press, 2005.

[34] S. F. Mason, C. R. Berger, S. Zhou, and P. Willet, "Detection, synchronization, and doppler scale estimation with multicarrier waveformsin underwater acoustic communications," IEEE J. Sel. Areas Commun., vol. 26, no. 9, pp. 1638-1649, 2008.

[35] B. Muquet, Z. Wang, and G. Giannakis, "Cyclic prefixing or zeropadding for wireless multicarrier transmissions?" IEEE Trans. Commun., vol. 50, no. 12, pp. 2136-2148, Dec. 2002.

[36] G. Leus and P. V. Walree, "Nonbinary LDPC coding for multicarrier underwater acoustic communication," IEEE J. Sel. Areas Commun., vol. 26, no. 9, pp. 1662-1673, Dec. 2008.

[37] J. Z. Huang, S. Zhou, J. Huang, C. R. Berger, and P. Willett, "Progressive inter-carrier interference equalization for OFDM transmission over timevarying underwater acoustic channels," in Proc. MTS/IEEE Oceans Conference, 2010.

[38] H. Sun, W. Shen, Z. Wang, S. Zhou, X. Xu, and Y. Chen, "Joint carrier frequency offset and impulse noise estimation for underwater acoustic OFDM with null subcarriers," in Proc. IEEE Oceans Conference, 2012.

[39] B. C. Kim and I. T. Lu, "Parameter study of OFDM underwater communications system," in Proc. MTS/IEEE Oceans, Providence, RI, 2000.

[40] M. Stojanovic, "OFDM for underwater acoustic communications: Adaptive synchronization and sparse channel estimation," ICASSIP, p. 5288, 2008. 
[41] J. Huang, S. Zhou, and P. Willett, "Nonbinary LDPC coding for multicarrier underwater acoustic communication," IEEE J. Sel. Areas Commun., vol. 26, no. 9, pp. 1684-1696, Dec. 2008.

[42] M. Stojanovic, "Low complexity OFDM detector for underwater channels," in Proc. MTS/IEEE OCEANS conference, Boston, 2006.

[43] F. Qu and L. Yang, "Basis expansion model for underwater acoustic channels," in Proc. MTS/IEEE OCEANS Conf, Quebec City, 2008.

[44] B. Li, S. Zhou, J. Huang, and P. Willett, "Scalable OFDM design for underwater acoustic communication," in ICASSP 2008, Las Vegas, Nevada, USA, 2008.

[45] B. Li, J. Huang, S. Zhou, K. Ball, M. Stojanovic, L. Freitag, and P. Willett, "MIMO-OFDM for high rate underwater acoustic OFDM systems," IEEE $J$. Ocean Eng, vol. 34, no. 4, Oct. 2009.

[46] T. Kand and R. A. Iltis, "Iterative carrier frequency offset and channel estimation for underwater acoustic OFDM systems," IEEE J. Sel. Areas Commun., vol. 26, no. 9, pp. 1650-1661, Dec. 2008.

[47] K. Tu, D. Fertonani, T. M. Duman, and P. Hursky, "Mitigation of intercarrier interfereence in OFDM systems over underwater acoustic channels," in Proc. MTS/IEEE OCEANS, Bremen, Germany, May 2009.

[48] F. Qu and L. Yang, "Rate and reliability oriented underwater acoustic communication schemes," in Proc. 2009 DSP \& SPE Workshop, Marco Island, FL, Jan. 2009.

[49] C. R. Berger, S. Zhou, J. Presig, and P. Willett, "Sparse channel estimation for multicarrier underwater acoustic communication: From subspace methods to compressed sensing," IEEE Trans. Signal Process., vol. 58, no. 3, pp. 1708 1721, Mar. 2010.

[50] P. Ceballos and M. Stojanovic, "Adaptive channel estimation and data detection for underwater acoustic MIMO OFDM systems," IEEE J. Ocean Eng., vol. 35, no. 3, pp. 635-646, Jul. 2010.

[51] T. Kang, H. C. Song, W. S. Hodgkiss, and J. S. Kim, "Long-range multicarrier acoustic communications in shallow water based on iterative sparse channel estimation," J. Acous. Soc. Amer., vol. 128, no. 6, Dec. 2010.

[52] Y. R. Zheng, C. Xiao, T. C. Yang, and W. B. Yang, "Frequency-domain channel estimation and equalization for shallow-water acoustic communications," Elsevier J. Phys. Commun., vol. 3, pp. 48-63, Mar. 2010. 
[53] P. J. Gendron, "Orthogonal frequency division multiplexing with on-offkeying: Noncoherent performance bounds, receiver design and experimental results," U. S. Navy Journal of Underwater Acoustics, vol. 56, no. 2, pp. 267-300, 2006.

[54] J. Huang, J.-Z. Huang, C. R. Berger, S. Zhou, and P. Willett, "Iterative sparse channel estimation and decoding for underwater MIMO-OFDM," in EURASIP J. on Adv. Signal Process., 2010.

[55] S. Mason, C. S. Berger, S. Zhou, and P. Willett, "Detection, synchronization, and doppler scale estimation with multicarrier waveforms in underwater acoustic communication," IEEE J. Sel. Areas Commun., vol. 26, no. 9, 2008.

[56] M. Chitre, S. H. Ong, and J. Potter, "Performance of coded OFDM in very shallow water channels and snapping shrimp noise," in Proc. MTS/IEEE OCEANS vol 2, 2005.

[57] C. R. Berger, W. Chen, S. Zhou, and J. Huang, "A simple and effective noise whitening method for underwater acoustic orthogonal frequency division multiplexing," J. Acoust. Soc. Amer., vol. 127, no. 4, pp. 2358-2367, 2008.

[58] X. Ma, C. Tepedelenlioglu, G. B. Giannakis, and S. Barbarossa, "Nondataaided carrier offset estimations for OFDM with null subcarriers: Identifiability, algorithms, and performance," IEEE J. Sel. Areas Commun., vol. 19, no. 12, pp. 2504-2515, 2001.

[59] S. Yerramalli and U. Mitra, "Optimal resampling of OFDM signals for multiscale - multilag underwater acoustic channels," IEEE J. Ocean. Eng., vol. 36, no. 1, pp. 126-138, 2011.

[60] R. Negi and J. Cioffi, "Pilot tone selection for channel estimation in a mobile OFDM system," IEEE Trans. Consumer Electronics, vol. 44, no. 3, Aug 1998.

[61] M. Tuchler, R. Koetter, and A. C. Singer, "Turbo equalization: Principle and new results," IEEE Trans. Commun., vol. 50, no. 5, pp. 754-766, 2002.

[62] M. Tuchler, A. C. Singer, and R. Koetter, "Minimum mean squared error equalization using a priori information," IEEE Trans. Signal Process., vol. 50, no. 3, pp. 673-683, 2002.

[63] Z. Wang, Z. Shengli, J. Catipovic, and P. Willett, "Asynchronous multiuser reception for OFDM in underwater acoustic communications," IEEE Transactions on Wireless Communications., vol. 12, no. 3, pp. 1050-1061, Mar. 2013.

[64] J. Ward, M. Fitzpatrick, N. Dimarzio, D. Moretti, and R. Morrizzey, "New algorithm for open ocean mammal monitoring," in Proc. MTS/IEEE OCEANS Conf., 2000. 
[65] J. Catipovic, "Robust acoustic communication with underwater vehicles in noisy and jammed shallow water environments," in OCEANS 1992: Mastering the Oceans Through Technology, 1992.

[66] J. Laster and J. Reed, "Interference rejection in digital wireless communications," IEEE Signal Process. Mag., vol. 14, no. 3, pp. 37-62, 1997.

[67] T. Zogakis, P. Chow, J. Aslanis, and J. Cioffi, "Impulse noise mitigation strategies for multicarrier modulation," in Proc. Int. Conf. Commun., 1993, pp. $784-788$.

[68] T. Li, W. H. Mow, and M. H. Siu, "A joint approach to erasure marking and Viterbi decoding for impulsive noise channels," in Proc. IEEE Workshop on Signal Process, 2003.

[69] S. Zhidkov, "Impulsive noise suppression in OFDM-based communication systems," IEEE Trans. Consum. Electron., vol. 49, no. 4, pp. 944-948, 2003.

[70] A. Mengi and A. Vinck, "Successive impulsive noise suppression in OFDM," in Proc. IEEE Int. Symp. on Power Line Commun. Appl., 2010.

[71] M. F.-G. García, J. Rojo-Álvarez, F. Alonso-Atienza, and M. MartínezRamón, "Support vector machines for robust channel estimation in OFDM," IEEE Signal Process. Lett., vol. 13, no. 7, pp. 397-400, 2006.

[72] X. Yu, P. Lin, Z. He, and W. Wu, "OFDM channel estimation with impulse noise cancellation," in Proc. Int. Conf. on Wireless Commun., 2007, pp. 330 333.

[73] G. Caire, T. Al-Naffouri, and A. Narayanan, "Impulse noise cancellation in OFDM: An application of compressed sensing," in Proc. IEEE Int. Symp. Inf. Theory, 2008, pp. 1293-1297.

[74] K. Pelekanakis, H. Liu, and M. Chitre, "An algorithm for sparse underwater acoustic channel identification under symmetric alpha-stable noise," in Proc. of MTS/IEEE OCEANS Conf., 2011.

[75] J. Armstrong and H. A. Suraweera, "Decision directed impulse noise mitigation for OFDM in frequency selective fading channels," in Proc. GLOBALCOM, 2004, pp. 3536-3540.

[76] X. Xu, S. Zhou, H. Sun, A. K. Morozov, and Y. Zhang, "Impulsive noise suppression in per survivor processing based DSSS systems," in Proc. IEEE Oceans Conference, St John's Newfoundland Canada, September 2014.

[77] R. Nilsson, F. Sjoberg, and J. LeBlanc, "A rank-reduced LMMSE canceller for narrowband interference suppression in OFDM-based system," IEEE Trans. Commun., vol. 51, no. 12, pp. 2126-2140, 2003. 
[78] D. Darsena, "Successive narrowband interference cancellation for OFDM systems," IEEE Commun. Lett., vol. 11, pp. 73-75, 2007.

[79] D. Darsena and F. Verde, "Successive NBI cancellation using soft decisions for OFDM systems," IEEE Signal Process. Lett., vol. 15, pp. 873-876, 2008.

[80] A. Jeremic, T. A. Thomas, and A. Nehorai, "OFDM channel estimation in the presence of interference," IEEE Trans. Signal Process., vol. 52, no. 12, pp. 3429-3439, 2004.

[81] M. Morelli and M. Moretti, "Channel estimation in OFDM systems with unknown interference," IEEE Trans. Wireless Commun., vol. 8, no. 10, pp. 5338-5347, 2009.

[82] M. Morelli and M. Moretti, "Improved decoding of BICM-OFDM transmissions plagued by narrowband interference," IEEE Trans. Wireless Commun., vol. 10, no. 1, pp. 20-26, 2011.

[83] T. Li, W. H. Mow, V. Lau, M. Siu, R. Cheng, and R. Murch, "Robust joint interference detection and decoding for OFDM-based cognitive radio systems with unknown interference," IEEE J. Sel. Areas Commun., vol. 25, no. 3, pp. 566-575, 2007.

[84] A. Gomaa, K. M. Z. Islam, and N. Al-Dhahir, "Two novel compressive-sensing algorithms for NBI detection in OFDM systems," in Proc. Int. Conf. on Acoust., 2010, pp. 3294-3297.

[85] T. Al-Naffouri, A. Quadeer, and G. Caire, "Impulsive noise estimation and cancellation in DSL using orthogonal clustering," in Proc. IEEE Int. Symp. Inf. Theory, 2011, pp. 2841-2845.

[86] K. Fazel, "Narrow-band interference rejection in orthogonal multicarrier spread-spectrum communications," in Third Annual International Conference on Universal Personal Communications, 1994, pp. 46-50.

[87] Y. Zhu, D. Guo, and M. L. Honig, "A message-passing approach for joint channel estimation, interference mitigation, and decoding," IEEE Trans. Wireless Commun., vol. 8, no. 12, pp. 6008-6018, 2009.

[88] F. He, X. Wang, T. Yang, B. Hu, and R. Liu, "Two blind algorithms of MMSE filter based on second-order statistics in MIMO systems with multiple interferences," in Wireless Communications and Signal Processing (WCSP), 2011.

[89] S. V. Zhidkov, "Performance analysis and optimization of OFDM receiver with blanking nonlinearity in impulsive noise environment," IEEE Trans. Vehicular Technology, vol. 55, no. 1, pp. 234-242, Jan. 2006Jan. 
[90] M. Ghosh, "Analysis of the effect of impulse noise on multicarrier and single carrier QAM systems," IEEE Trans. Commun., vol. 44, no. 2, pp. 145-147, 1996.

[91] S. J. Johnson, Iterative Error Correction: Turbo, Low Density Parity-Check and Repeat Accumulate Codes. New South Wales: Cambridge University Press, 2010.

[92] J. H. Winters, "Optimum combining in digital mobile radio with cochannel interference," no. 2, 1984, pp. 528-539.

[93] M. Chiani, M. Z. Win, A. Zanella, R. K. Mallik, and J. H. Winters, "Bounds and approximations for optimum combining of signals in the presence of multiple cochannel interferers and thermal noise," IEEE Transactions on Communications, vol. 51, no. 2, pp. 296-306, Feb. 2003.

[94] M. Stojanovic, J. A. Catipovic, and J. G. Proakis, "Adaptive multichannel combining and equalization for underwater acoustic communications," $J$. Acoust. Soc. Amer., vol. 94, no. 3, pp. 1621-1631, 1993.

[95] S. Roy, T. M. Duman, V. McDonald, and J. G. Proakis, "High rate communication for underwater acoustic channels using multiple transmitters and space-time coding: Receiver structures and experimental results," IEEE J. Ocean. Eng., vol. 32, no. 3, pp. 663-688, Jul. 2007.

[96] H.-C. Song, "Long-range acoustic communication in deep water using a towed array: Beam diversity," in OCEANS, Yeosu 2012.

[97] R. P. Goddard, The Sonar Simulation Toolset, Release 4.1: Science, Mathematics and Algorithms. Seattle, WA: APL-UW TR 0404, Applied Physics Laboratory University of Washington, April 2004.

[98] P. Qarabaqi and M. Stojanovic, "Statistical characterization and computationally efficient modeling of a class of underwater acoustic communication channels," IEEE Journal of Oceanic Engineering, vol. 38, no. 4, pp. 701-717, Oct. 2013. 


\section{APPENDIX}

\section{AUTEC Experiment in May 2014 (AUTEC-0514)}

\section{A.1 Description of the AUTEC May 2014 Experiment}

During May 2014, experiments were conducted at AUTEC to test the interference mitigation algorithms. A multichannel projector was configured for multichannel simultaneous transmission of both interfering and desired acoustic waveforms. The desired OFDM signal consisted of a channel probe, four data packets containing identical messages and a final channel probe while LFM waveforms of various time durations and bandwidths provided the interference. Receptions of the transmitted waveforms were recorded on more than forty distributed oceanbottom hydrophones.

The simultaneous transmission of interfering and desired acoustic waveforms was accomplished by playing stereo wavefiles ("songs") created using MATLAB with the output selected to the desired AUTEC transmitter. For AUTEC-0514, interference was transmitted from node 69 and the OFDM signal was transmitted from node 75. Figure A.1 shows the receivers in the vicinity of nodes 69 and 75. Data from receivers $67,68,76,78$, and 82 was processed as part of the analysis of AUTEC-0514. Nine different songs of interfering signals of various bandwidths and time durations were transmitted contemporaneously with the same OFDM communication signal. Table A.1 lists the sequentially played songs. Each song is divided into 17 transmit time slots of 5.6827 second duration. During the first three time slots, only the communication signal was transmitted to allow for processing of the received signals under noise only conditions. From the fourth time slot until the end of the song, both the interfering signal and desired signal were transmitted. Figure A.2 shows the transmitted time series from two such time slots to illustrate the transmission schedule of the interference and desired signal. The interference, 


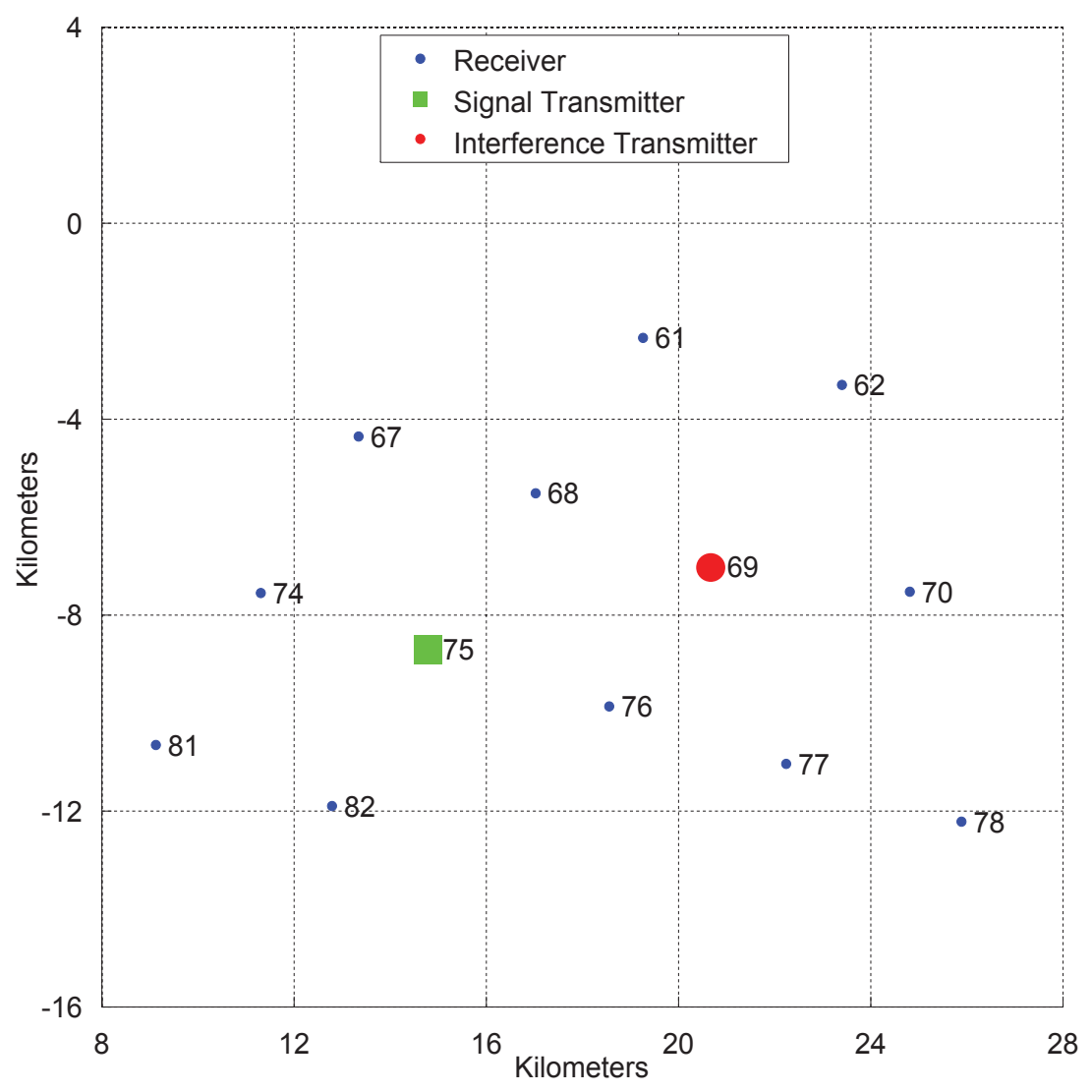

Figure A.1: Relative position of transmitters and receivers in the AUTEC-0514 experiment.

shown in red, is a LFM pulse with a bandwidth of $750 \mathrm{~Hz}$ and duration of 21 milliseconds. The OFDM signal appears in blue. Nine interfering signals were transmitted during each time slot at a repetition rate of 420.7 milliseconds which equals to the OFDM symbol duration plus guard interval $T+T_{g}$. The transmission of the first four interfering signals was synchronized to the start of the OFDM blocks within the packet. The fifth interfering signal was transmitted synchronous to the final packet probe. The last four interfering signals were transmitted alone. 


\begin{tabular}{|c|c|c|c|c|c|}
\hline Track & File name & Time & \multicolumn{2}{|c|}{$\begin{array}{c}\text { Interference } \\
\text { (M:SS) }\end{array}$} & $\begin{array}{c}\text { Number of } \\
\text { Pandwidth }\end{array}$ \\
\hline 1 & Duration & Packets \\
2 & li_lfm_750BW_21Tmsec_int_li_ofdm & $1: 36$ & $750 \mathrm{~Hz}$ & $21 \mathrm{~ms}$ & 17 \\
3 & li_lfm_750BW_43Tmsec_int_li_ofdm & $1: 36$ & $750 \mathrm{~Hz}$ & $43 \mathrm{~ms}$ & 17 \\
4 & li_lfm_1500BW_21Tmsec_int_li_ofdm & $1: 36$ & $750 \mathrm{~Hz}$ & $85 \mathrm{~ms}$ & 17 \\
5 & li_lfm_1500BW_43Tmsec_int_li_ofdm & $1: 36$ & $1500 \mathrm{~Hz}$ & $21 \mathrm{~ms}$ & 17 \\
6 & li_lfm_1500BW_85Tmsec_int_li_ofdm & $1: 36$ & $1500 \mathrm{~Hz}$ & $43 \mathrm{~ms}$ & 17 \\
7 & li_lfm_3000BW_21Tmsec_int_li_ofdm & $1: 36$ & $1500 \mathrm{~Hz}$ & $85 \mathrm{~ms}$ & 17 \\
8 & li_lfm_3000BW_43Tmsec_int_li_ofdm & $1: 36$ & $3000 \mathrm{~Hz}$ & $21 \mathrm{~ms}$ & 17 \\
9 & li_lfm_3000BW_85Tmsec_int_li_ofdm & $1: 36$ & $3000 \mathrm{~Hz}$ & $43 \mathrm{~ms}$ & 17 \\
\hline
\end{tabular}

Table A.1: OFDM signal with LFM interference wavefiles.

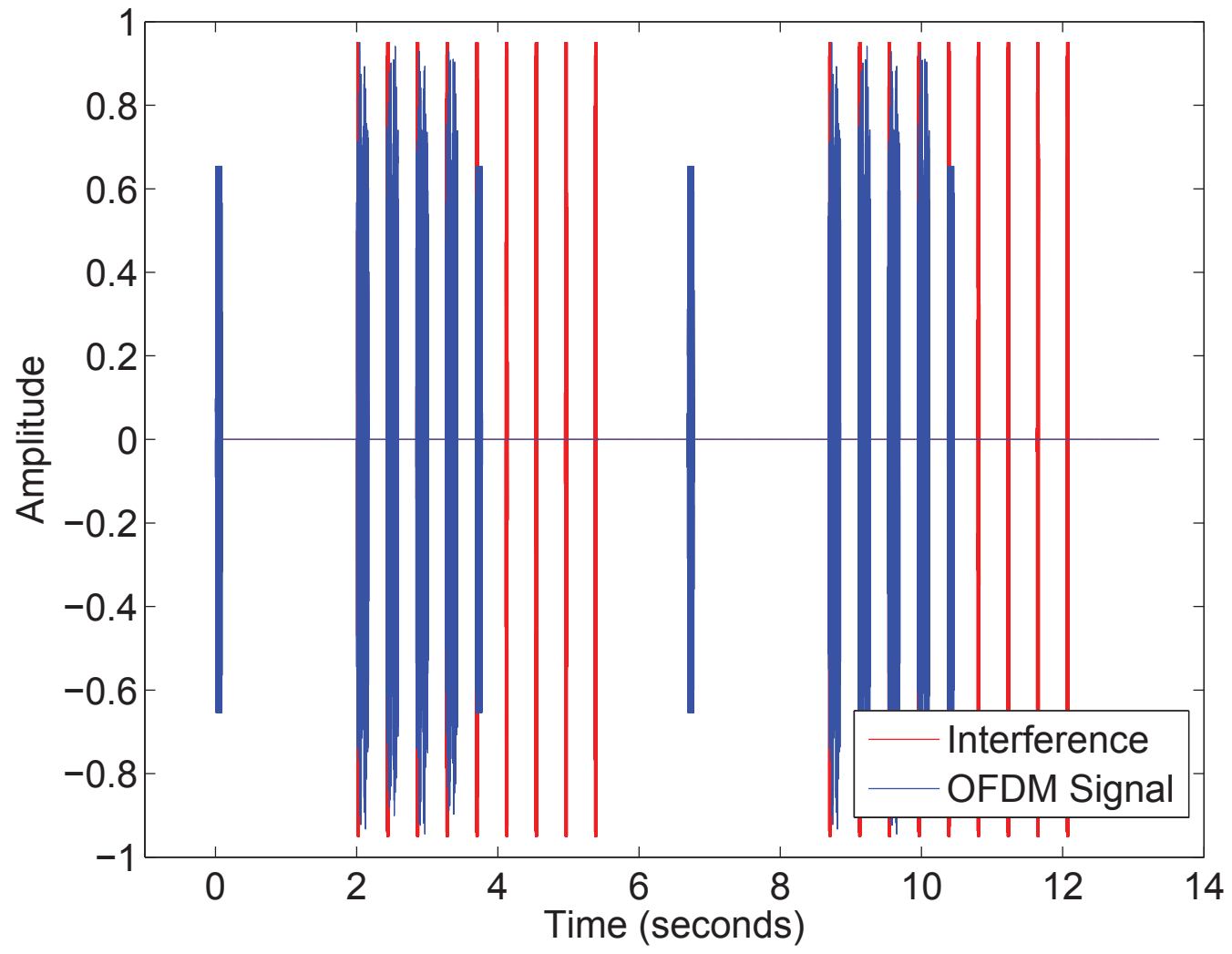

Figure A.2: Representative time series of the transmitted LFM interference and OFDM signal in the AUTEC-0514 experiment. 


\begin{tabular}{|l|l|l|}
\hline \multicolumn{2}{|l|}{ OFDM Packet LFM Probe Parameters } & Value \\
\hline Center Frequency & $f_{c, P}$ & $11 \mathrm{kHz}$ \\
\hline Sweep Direction & & Down \\
\hline Bandwidth & $B_{P}$ & $6.0 \mathrm{kHz}$ \\
\hline Duration & $T_{P}$ & $100 \mathrm{~ms}$ \\
\hline \multicolumn{2}{|c|}{ OFDM Parameters } & Value \\
\hline Center frequency & $f_{c}$ & $11 \mathrm{kHz}$ \\
\hline Bandwidth & $B$ & $6.0 \mathrm{kHz}$ \\
\hline \# of subcarriers & $K$ & 1024 \\
\hline \# data subcarriers & $\mid \mathcal{S}_{D}$ & 672 \\
\hline \# pilot subcarriers & $\mathcal{S}_{P} \mid$ & 256 \\
\hline \# null subcarriers & $\left|\mathcal{S}_{N}\right|$ & 256 \\
\hline Symbol Duration & $T$ & $170.7 \mathrm{~ms}$ \\
\hline Subcarrier spacing & $\Delta f=1 / \mathrm{T}$ & $5.859 \mathrm{~Hz}$ \\
\hline Guard interval & $T_{g}$ & $250 \mathrm{~ms}$ \\
\hline Symbol Constellation & & $\mathrm{BPSK}$ \\
\hline Blocks Per Packet & $N_{b l k}$ & 4 \\
\hline Blanking Period Between Packets & & $2.9 \mathrm{sec}$ \\
\hline \multicolumn{2}{|c|}{ LFM Interference Parameters } & Value \\
\hline Center Frequency & $f_{c, I}$ & $14 \mathrm{kHz}$ \\
\hline Sweep Direction & & $\mathrm{Up}$ \\
\hline Bandwidth & $B_{I}$ & $B / 8, B / 4, B / 2$ \\
\hline Duration & $T_{I}$ & $T / 8, T / 4, T / 2$ \\
\hline Repetition Rate & $R_{I}$ & $T+T g=420.7 \mathrm{~ms}$ \\
\hline
\end{tabular}

Table A.2: AUTEC-0514 OFDM and LFM transmit parameters.

\section{A.1.1 Generation of Pseudo-Experimental Data}

All of the received data packets from receivers 67, 68, 76, 78 and 82 were processed. The bandpass samples from the 1,752 received OFDM blocks in which the symbols were all detected correctly prior to decoding were set aside for use as received signals. The noise variance outside of the interference band in each block was calculated as:

$$
\hat{\sigma}_{\text {noise }}^{2}=\mathbb{E}_{\left\{m \in \mathcal{S}_{N} \cap \overline{\mathcal{S}}_{v}\right\}}\|z[m]\|^{2} .
$$

The signal power in each block was estimated as:

$$
\hat{P}_{\text {sig }}=\mathbb{E}_{\left\{m \in \mathcal{S}_{P} \cap \mathcal{S}_{D} \cap \overline{\mathcal{S}}_{v}\right\}}\|z[m]\|^{2}-\hat{\sigma}_{\text {noise }}^{2} .
$$

and the SNR in the block was then calculated as

$$
\left.\mathrm{SNR}_{\text {blk }}=10 \log _{10}\left(\hat{P}_{\text {sig }} / \hat{\sigma}_{\text {noise }}^{2}\right)\right)-10 \log _{10}\left(K f_{\text {sym }}\right)
$$

where $f_{\text {sym }}$ is the symbol rate. 
Sea noise was extracted from the received data packets at the locations shown in panel (a) of Fig. A.3. After verifying the bandpass noise $\tilde{\mathbf{w}}$ was free of interference, it was processed using the OFDM symbol block processing string shown in panel (b) of Fig. A.3 to produce complex frequency domain noise w . The variance of the noise $\mathbf{w}$ was estimated using Eq. A.1, that is

$$
\hat{\sigma}_{\mathbf{w}}^{2}=\mathbb{E}_{\left\{m \in \mathcal{S}_{N} \cup \overline{\mathcal{S}}_{v}\right\}}\|w[m]\|^{2}
$$

The original bandpass noise $\tilde{\mathbf{w}}$ was scaled so that after OFDM symbol processing its basebanded frequency domain counterpart $\mathbf{w}$ would have unit variance. The scaled bandpass noise vector $\tilde{\mathbf{w}}_{s}=\tilde{\mathbf{w}} / \hat{\sigma}_{\mathbf{w}}$ was saved.

Interference was obtained from the direct path receptions of the packet probe using the steps shown in panel (a) of Fig. A.4. Examples of the extracted signals from various points in the processing chain are shown below in panel (b). The portion of the channel probe $\tilde{\mathbf{v}}_{1}$ sweeping from 9.5 to $12.5 \mathrm{kHz}$ was selected as shown in red in panel (b). The time duration of this portion of the probe is 50 milliseconds and contains 5,485 samples. A Tukey window $\mathcal{W}_{\text {Tuk }}$ with roll-off factor of 0.25 was applied to the extracted portion of the probe producing $\tilde{\mathbf{v}}_{1, w}=$ $\mathcal{W}_{\text {Tuk }} \tilde{\mathbf{v}}_{1}$. Fifty milliseconds of interference-free sea noise $\tilde{\mathbf{w}}_{1}$ was extracted from a nearby portion of the received packet shown in black and a complementary window, $\mathcal{W}_{c}=1-\mathcal{W}_{\text {Tuk }}(n)$ was applied to the extracted noise producing $\tilde{\mathbf{w}}_{1, w}=\mathcal{W}_{\mathrm{c}} \tilde{\mathbf{v}}_{1}$. The windowed probe and sea noise were added resulting in a vector of fared interference and noise samples $\mathbf{v}_{1, f}=\tilde{\mathbf{v}}_{1, w}+\tilde{\mathbf{w}}_{1, w}$ shown in pink. A block of sea noise $\tilde{\mathbf{u}}$ of the same length as an OFDM received signal block and guard interval (16,433 samples) was extracted from another nearby portion of the received packet as indicated in blue. No windowing was applied to this noise. Samples 2,001 to 7,484 in $\tilde{\mathbf{u}}$ were replaced with by the vector of fared interference and noise forming $\tilde{\mu}$. To be clear, 


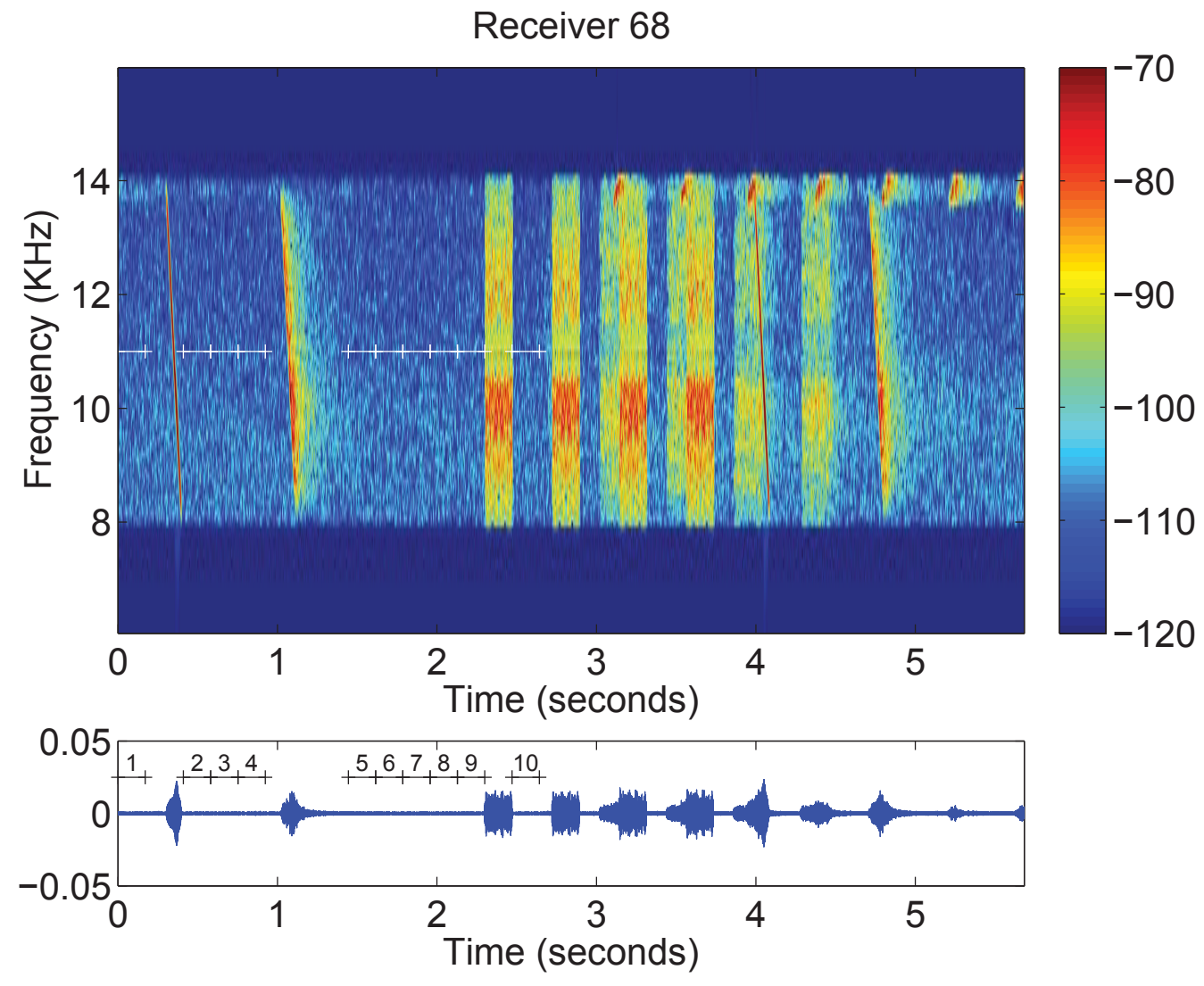

(a)

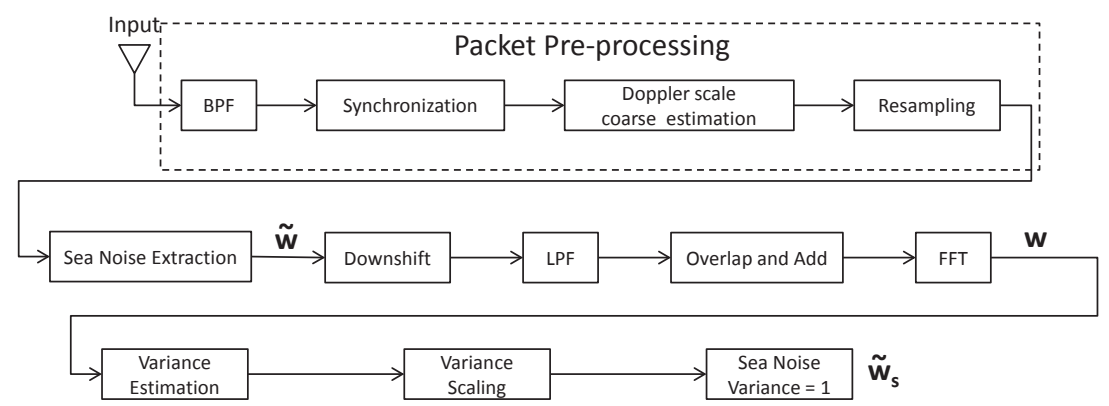

(b)

Figure A.3: Processing to extract sea noise from received data packets. 
in pseudo-code $\tilde{\mu}$ is formed by,

$$
\begin{aligned}
\tilde{\mu} & =\tilde{\mathbf{u}} \\
\tilde{\mu}(2001: 7484) & =\tilde{\mathbf{v}}_{1, f} .
\end{aligned}
$$

The resulting signal $\tilde{\mu}$ was processed using the OFDM processing string and the power of the interference was estimated as

$$
\hat{P}_{\text {int }}=\mathbb{E}_{\left\{m \in \mathcal{S}_{N} \cap \mathcal{S}_{v}\right\}}\|z[m]\|^{2}-\hat{\sigma}_{\text {noise }}^{2} .
$$

where the noise estimate $\hat{\sigma}_{\text {noise }}^{2}$ was calculated using Eq. A.1. The faired interference plus noise vector $\mathbf{v}_{1, f}$ and the extracted block of sea noise were scaled using the same scale factor $\phi=\sqrt{\hat{P}_{\text {int }}}$ to produce the scaled interference vector $\tilde{\mathbf{v}}_{s}=\phi \mathbf{v}_{1, f}$ and scaled noise vector $\tilde{\mathbf{u}}_{s}=\phi \tilde{\mathbf{u}}$. Fig. A.5 shows the extracted faired interference $\tilde{\mathbf{v}}_{s}$ inserted into a block of sea noise $\tilde{\mathbf{u}}_{s}$ at an arbitrary start time. The 819 sets of vectors $\left\{\tilde{\mathbf{v}}_{s}, \tilde{\mathbf{u}}_{s}\right\}$ were saved.

The MATLAB ${ }^{\circledR}$ code in Section A.2 combines the error free received data blocks $(1,752)$, scaled noise blocks $(4,868)$, and interference and noise pairs $(819)$ to test the algorithms at various SNR and SIR levels. Fig. A.6 shows an example of two OFDM blocks synthesized from the experimental data suffering noise and time orthogonal interference. 


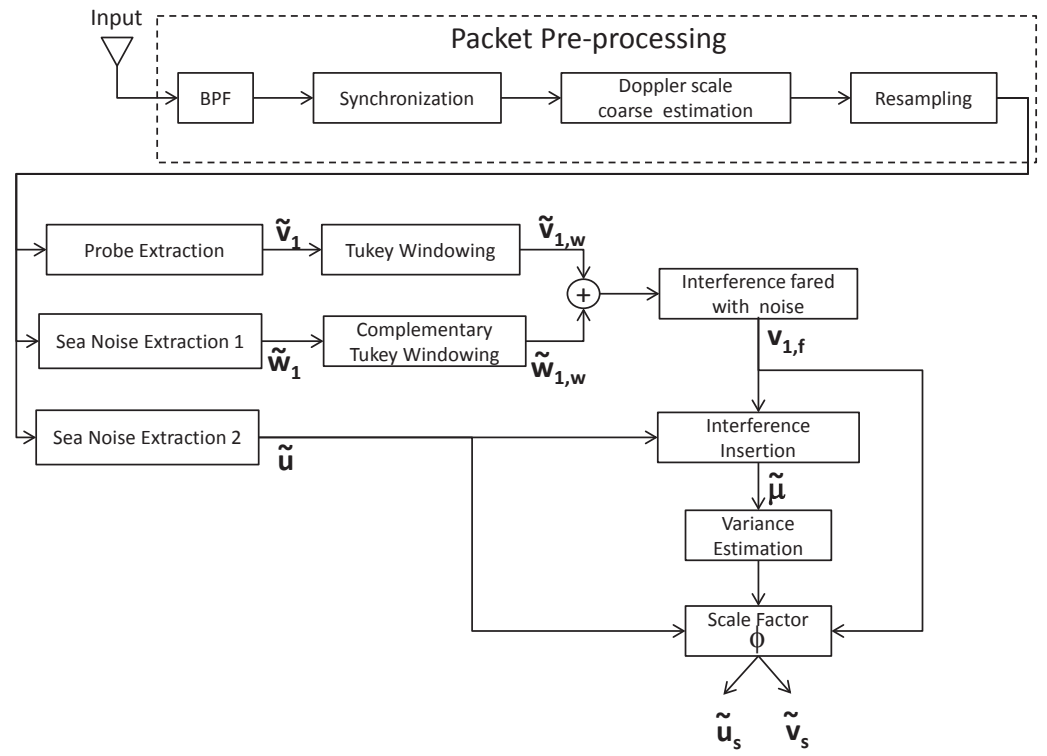

(a)

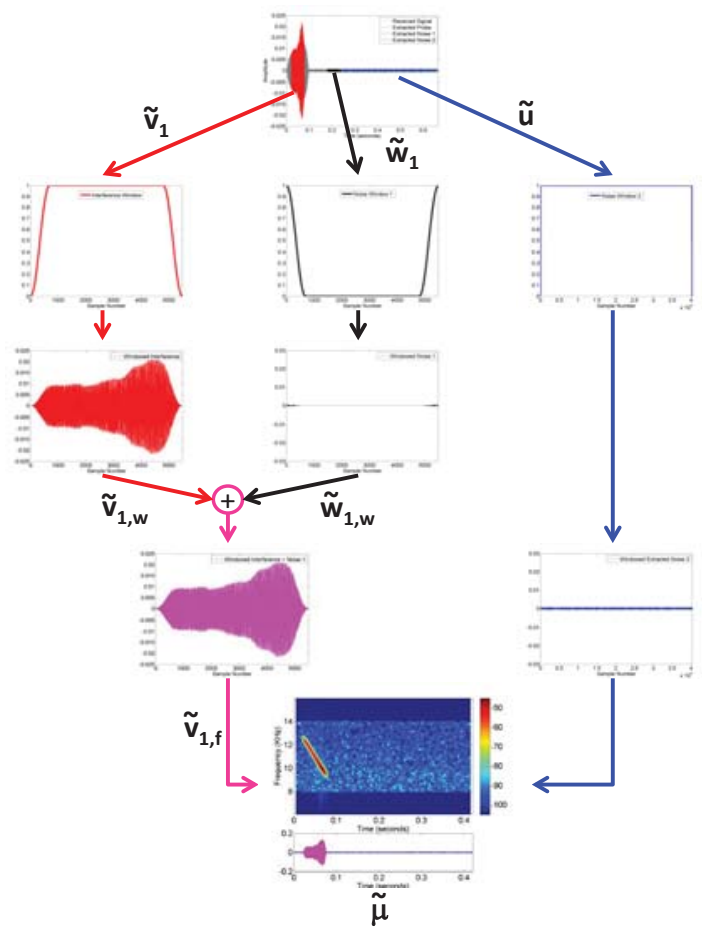

(b)

Figure A.4: Processing to extract interference and sea noise from received data packets.

The block diagram of the processing chain is shown in panel (a). Examples of the extracted signals from various points in the process chain are shown below in panel (b). 


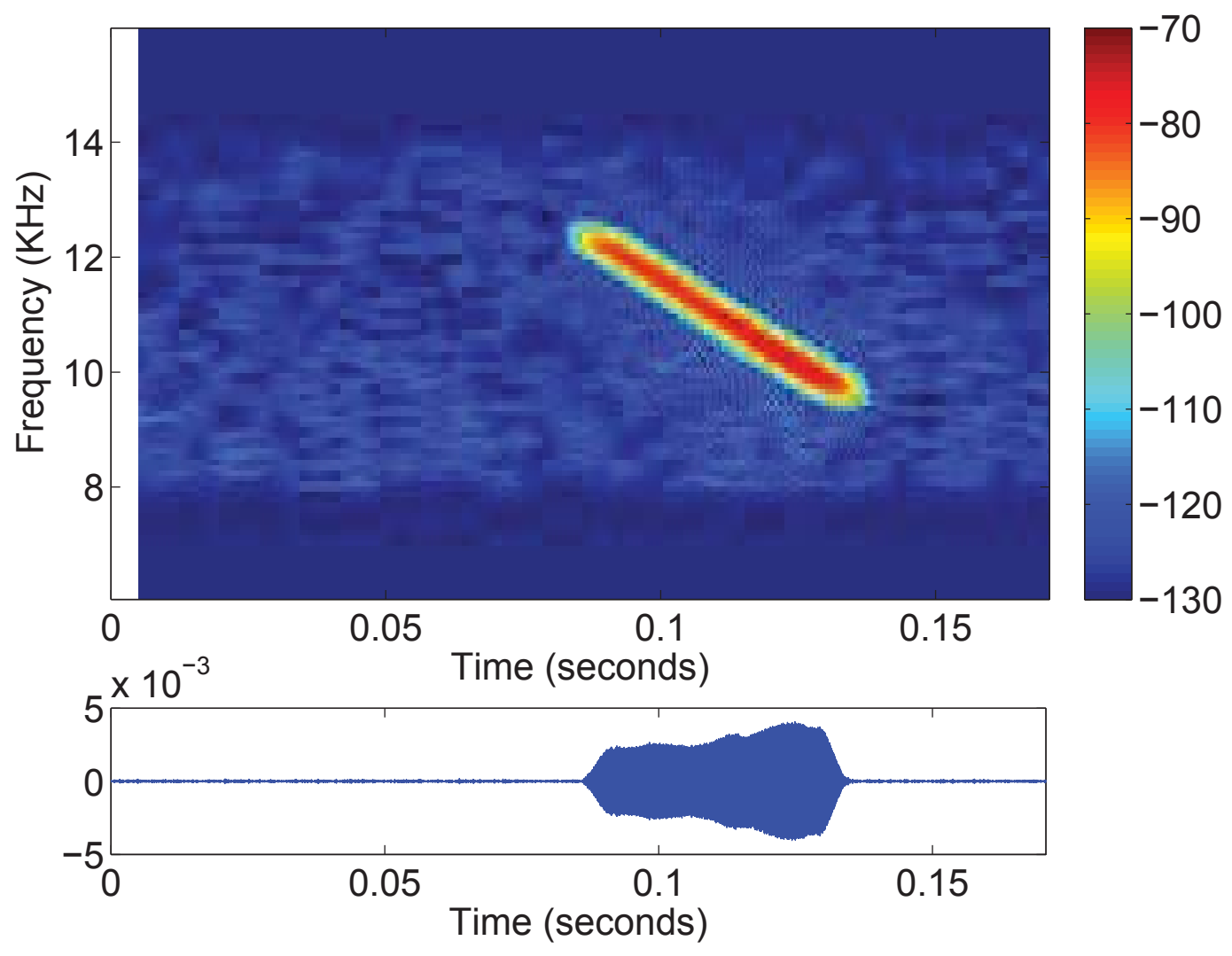

Figure A.5: Interference extracted from channel probe and faired into background noise.
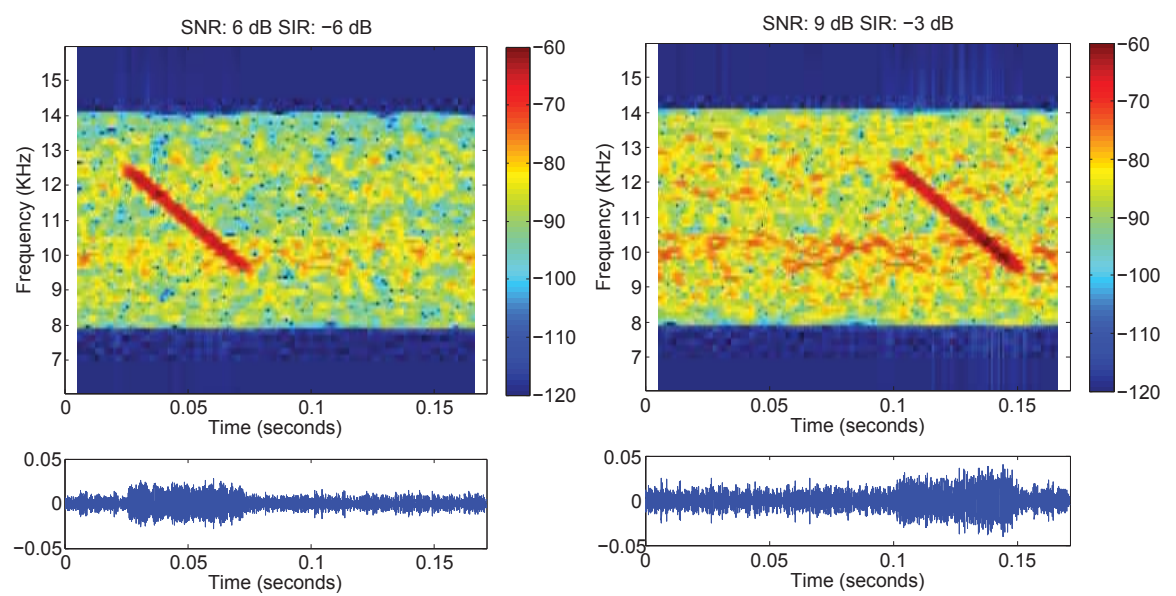

(a)

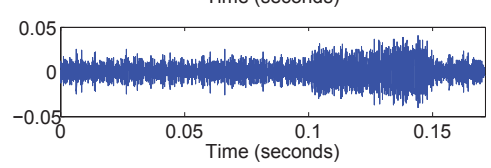

(b)

Figure A.6: Two examples of synthesized experimental data with time orthogonal interference. 


\section{A.2 MATLAB ${ }^{\circledR}$ Code}

The following pages contain the MATLAB ${ }^{\circledR}$ code used for testing the algorithms. 


\section{MAIN_generate_2channel_timeorthogonal_testcase.m}

This script generates a 2 receiver test case with time orthogonal interference.

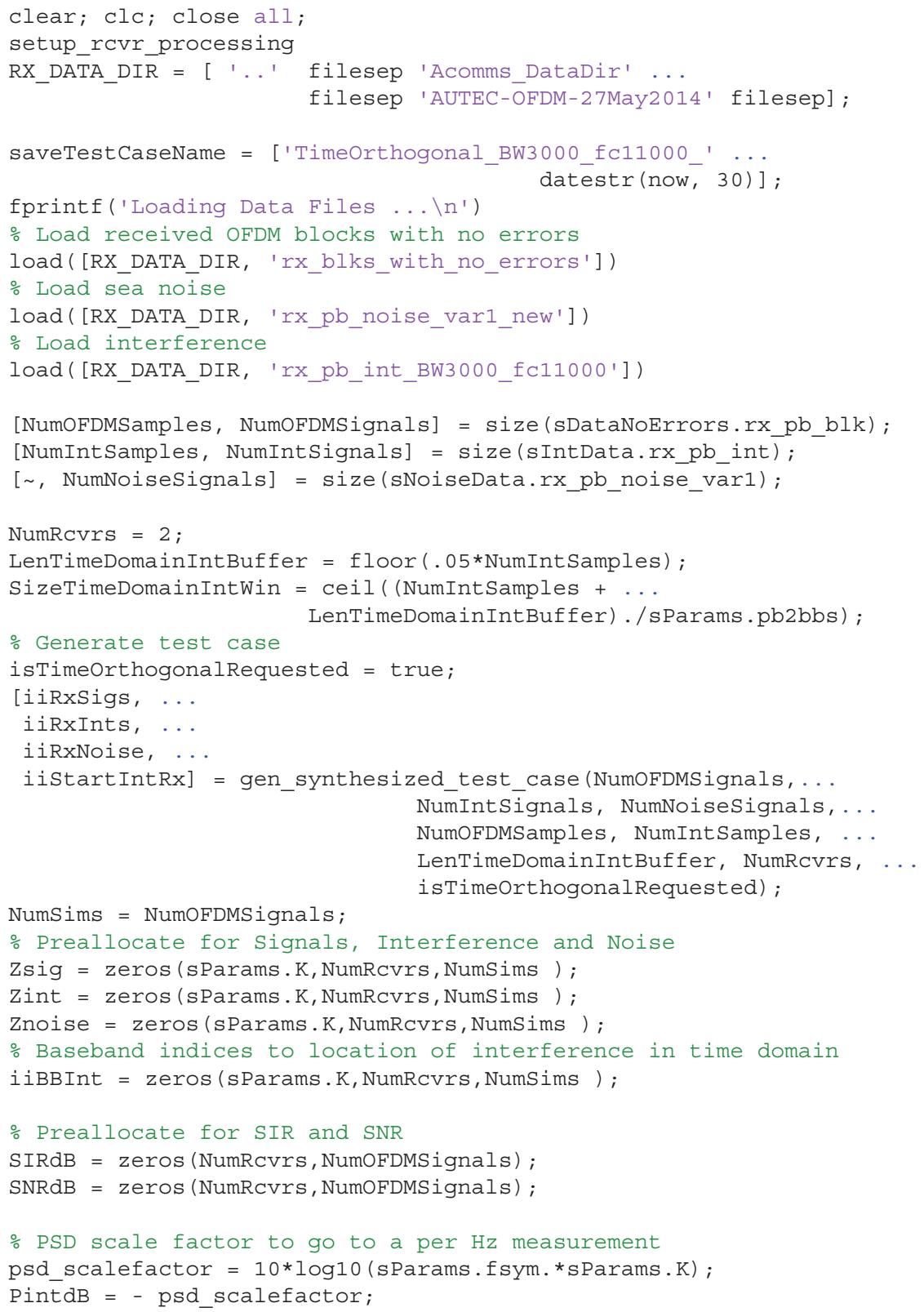




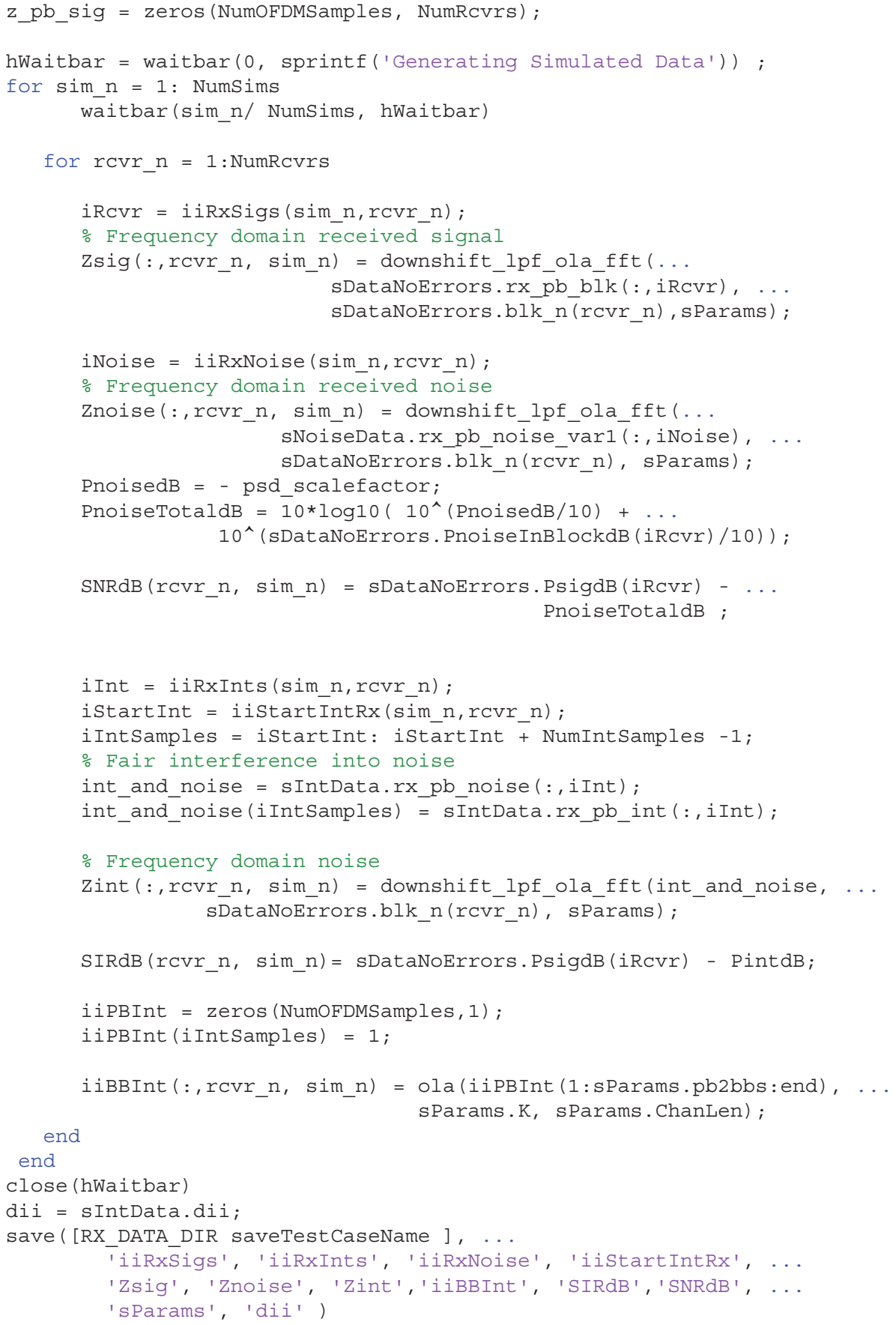




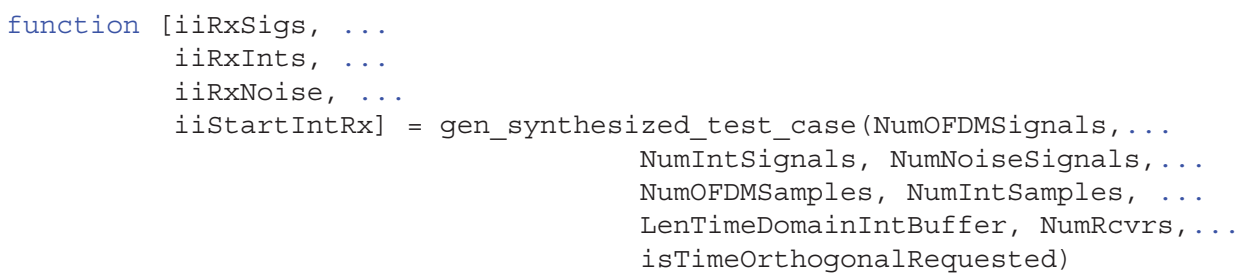

\section{gen_synthesized_test_case.m}

PURPOSE: Combine error-free OFDM received signals, sea noise and interference to generate a synthisized test case for the number of receivers specified by NumRcvrs. The grouping of received signals, noise and interference is unique for each receiver combination.

INPUT: NumOFDMSignals $=$ Number of error-free OFDM received signals $(z)$

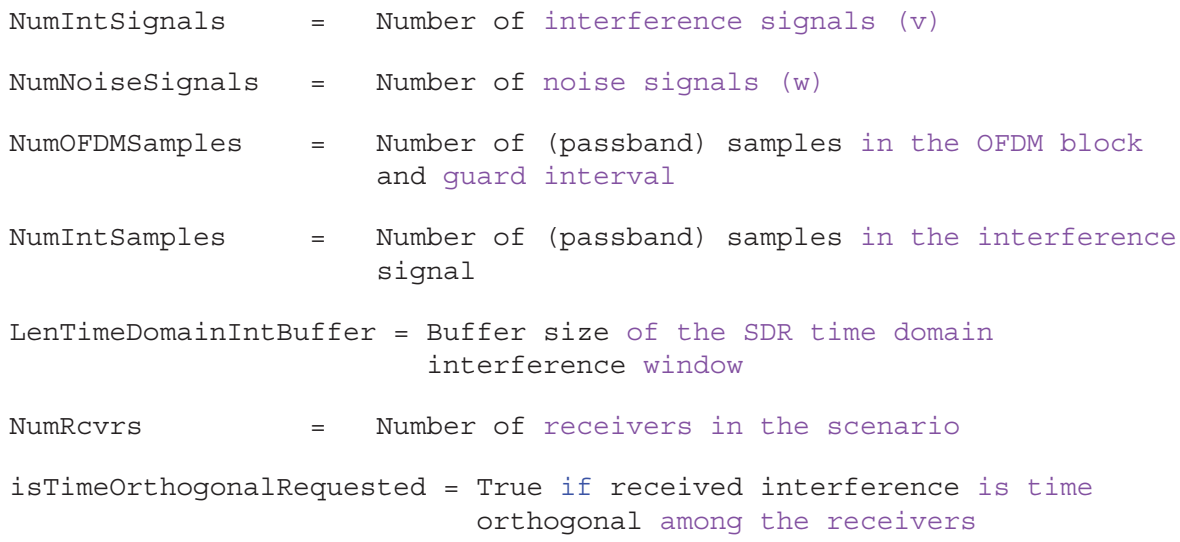

OUTPUT: iiRxSigs $=$ indices into the stored OFDM signals (z) [NumOFDMSignals x NumRcvrs]

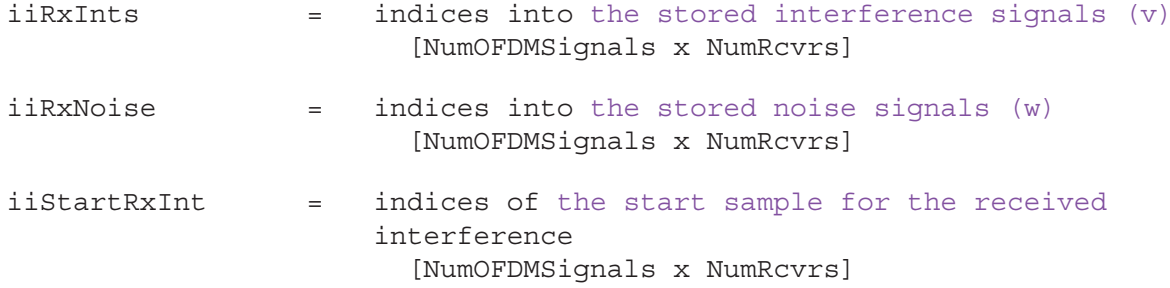

NOTES: The received data from AUTEC0514 resulted in NumOFDMSignals $=1752$ NumNoiseSignals $=4868$ NumIntSignals $=819$ The code generates a test case of size [NumOFDMSignals $\mathrm{x}$ NumRcvrs] by reusing the signals to create the desired number of realizations. In order to provide independence, no signals are not repeated within a given realization. That is, no indices are repeated across the rows of iiRxSigs, iiRxInts, iiRxNoise, iiStartRxInt.

- Signal indices 


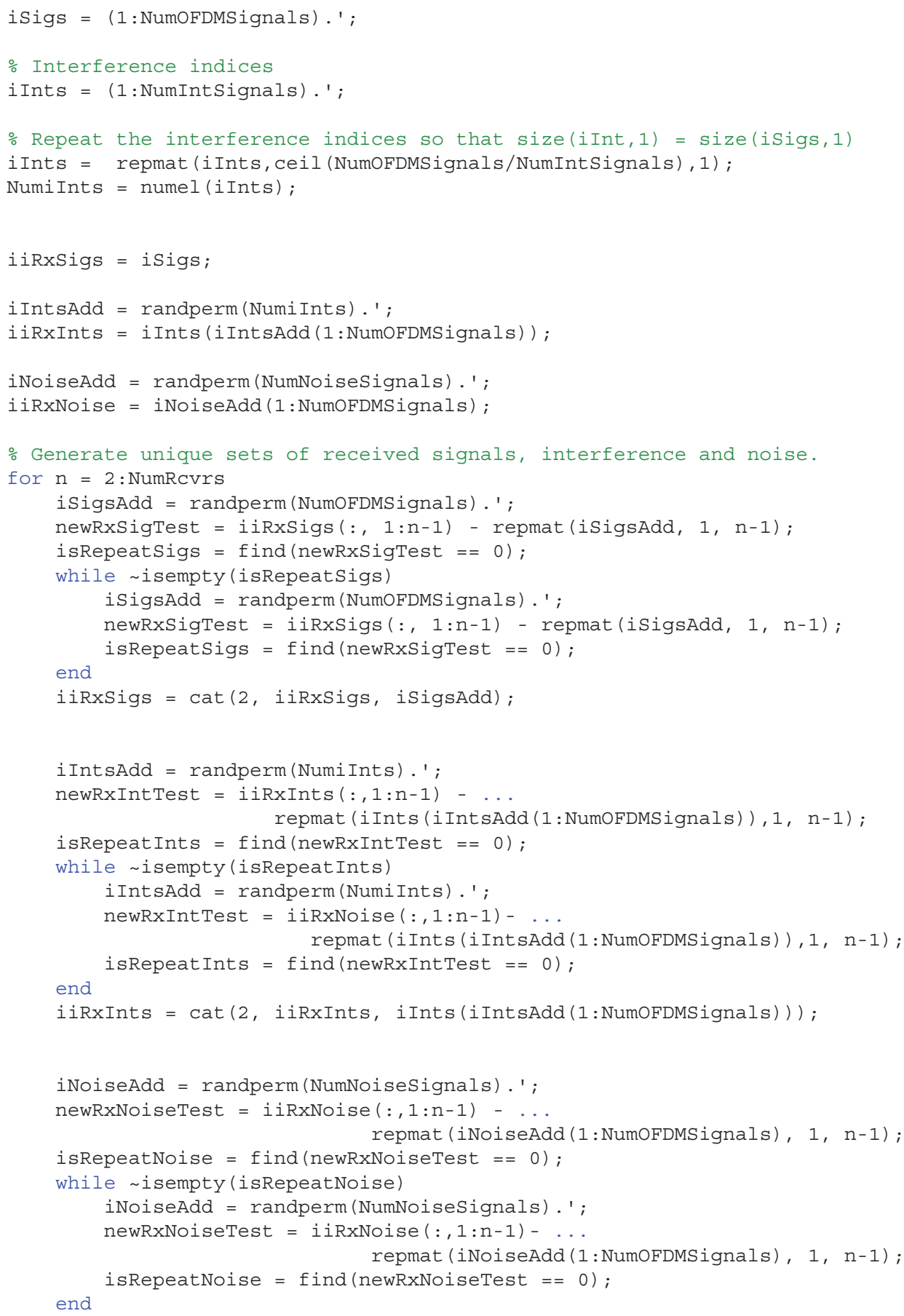


iiRxNoise = $\operatorname{cat}(2$, iiRxNoise, iNoiseAdd(1:NumOFDMSignals));

end

\% Generate the start times of the interference within each received signal LenTimeDomainIntWin = NumIntSamples + LenTimeDomainIntBuffer;

isTimeOrthogonalPossible = NumOFDMSamples/NumRcvrs - LenTimeDomainIntWin $>0$;

istimeOrthogonalRequested = true;

if isTimeOrthogonalPossible \&\& isTimeOrthogonalRequested

\%iWins = linspace $(1$, NumOFDMSamples, NumRcvrs+1);

iWins $=$ round $($ Iinspace $(1$, NumOFDMSamples, NumRcvrs+1));

istartWins $=$ iWins $(1:$ NumRcvrs $) ;$

iistartIntRx = zeros (NumOFDMSignals, NumRcvrs);

LenTimeDomainstartWin = iStartWins (2) - LenTimeDomainIntWin;

for revr_n $=1:$ NumRevrs

iistartIntRx $(:, \operatorname{rCv} r \mathrm{n})=\ldots \ldots$

ceil (LenTimeDomainStartWin. *rand (NumofDMSignals, 1)) . .

end

+ istartWins (rcvr_n) - 1;

else

iistartIntRx = zeros (NumOFDMSignals, NumRcvrs);

for rcvr_n $=1$ : NumRcvrs

iistartIntRx $(:, r$ CVr_n $)=\operatorname{ceil}(\ldots$

end

(NumOFDMSamples-NumIntSamples) . *rand (NumOFDMSignals, 1)) ;

end

Published with MATLAB ${ }^{R} 2014 a$ 


\section{MAIN_run_sdr_synthetic_simulation.m}

This script runs testcases generated using MAIN_generate_2channel_timeorthogonal_testcase.m MAIN_gen_3channel_timeorthogonal_testcase_AGWN_impulse_channel.m

MAIN_generate_2channel_timeorthogonal_testcase_sim_noise.m

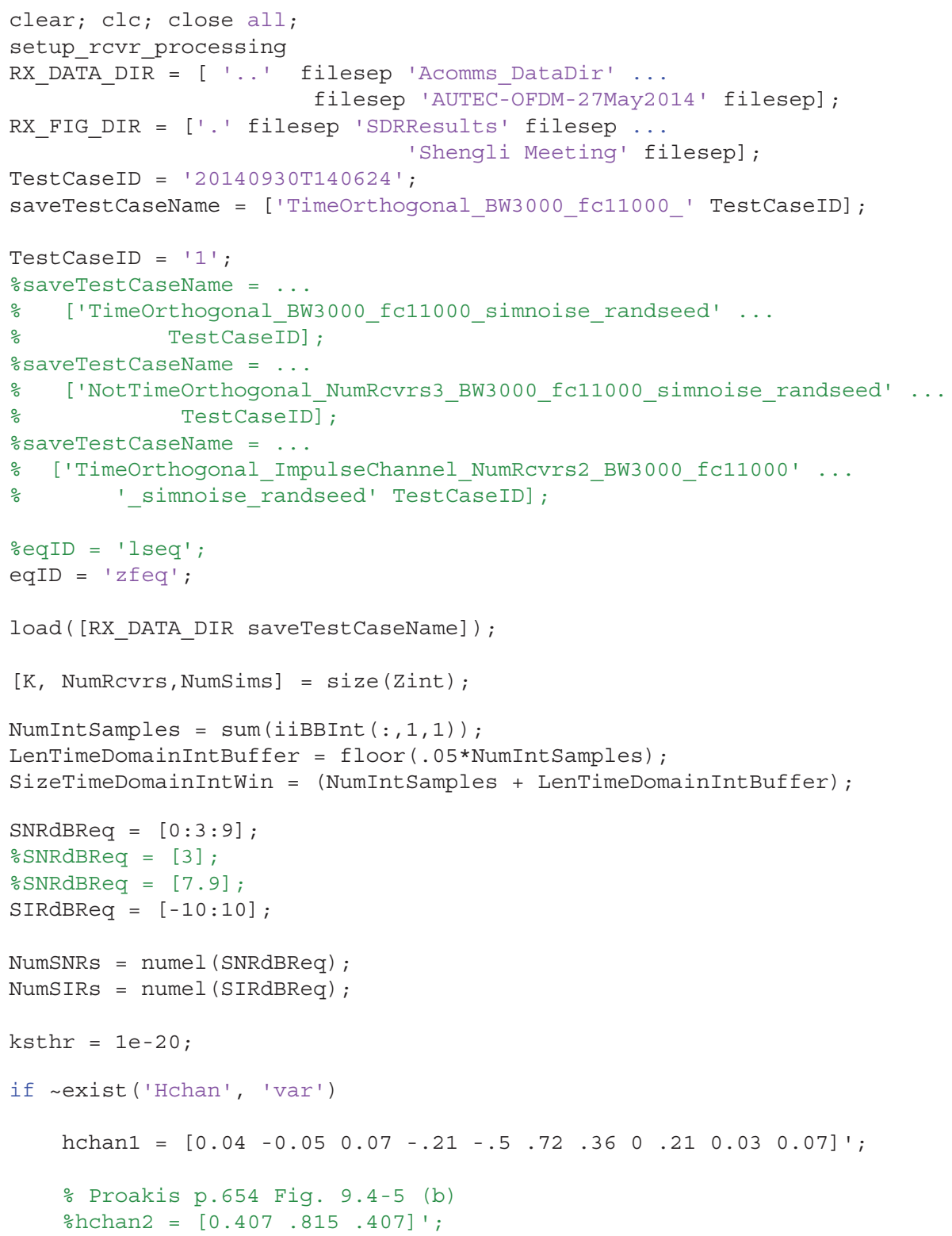




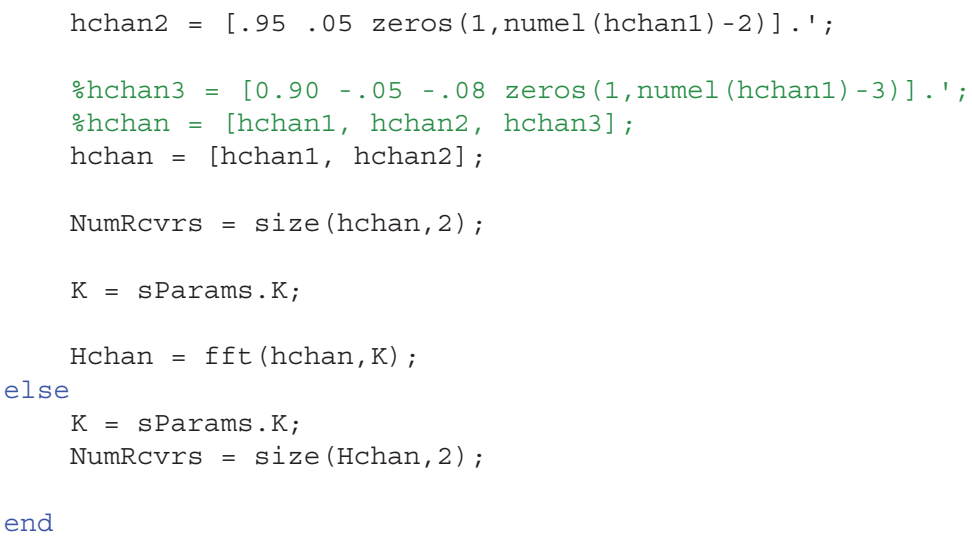




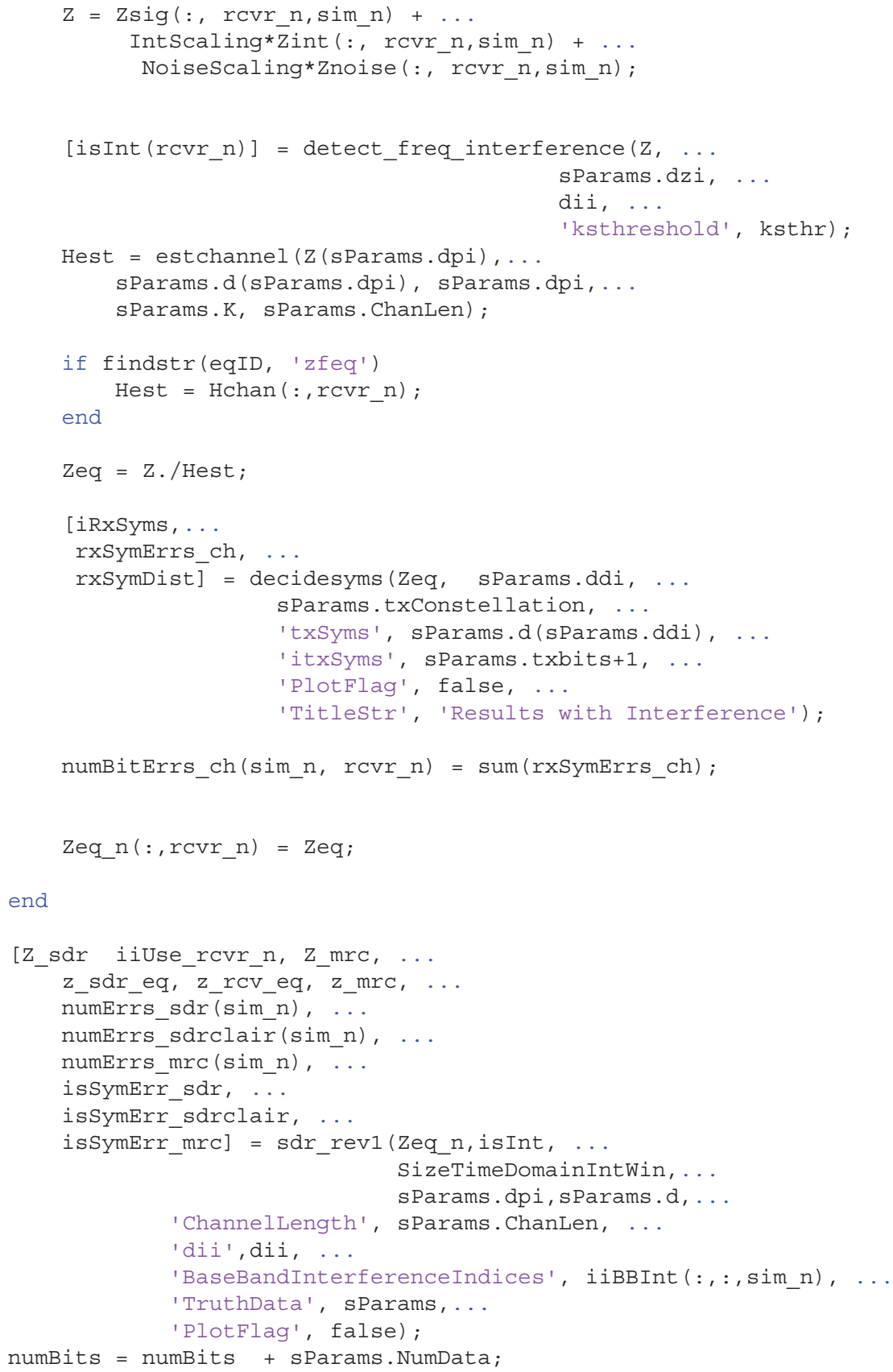




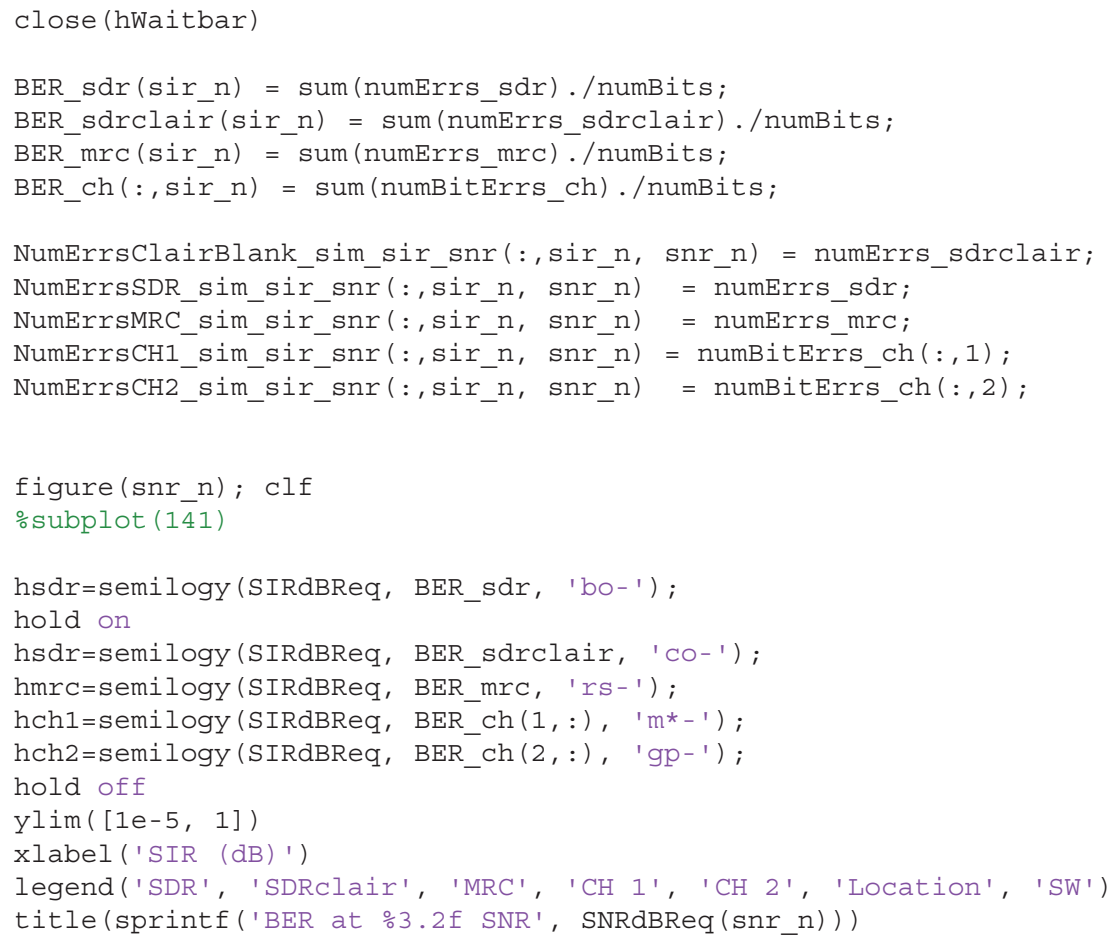

Published with MATLAB® R2014a 


\section{BIBLIOGRAPHY}

"Grand bahama area code information," http://www.areacodehelp.com/acsa/ grand_bahama_area_code.shtml, accessed: 2015-02-22.

"Submarine signaling," Scientific American Supplement, no. 2071, pp. 168-170, September 1915.

Al-Naffouri, T., Quadeer, A., and Caire, G., "Impulsive noise estimation and cancellation in DSL using orthogonal clustering," in Proc. IEEE Int. Symp. Inf. Theory, 2011, pp. 2841-2845.

Armstrong, J. and Suraweera, H. A., "Decision directed impulse noise mitigation for OFDM in frequency selective fading channels," in Proc. GLOBALCOM, 2004, pp. 3536-3540.

Baggeroer, A., "Acoustic telemetry - an overview," IEEE Journal Oceanic Eng., vol. 9, no. 4, pp. 229-235, 1984.

Berger, C. R., Chen, W., Zhou, S., and Huang, J., "A simple and effective noise whitening method for underwater acoustic orthogonal frequency division multiplexing," J. Acoust. Soc. Amer., vol. 127, no. 4, pp. 2358-2367, 2008.

Berger, C. R., Zhou, S., Presig, J., and Willett, P., "Sparse channel estimation for multicarrier underwater acoustic communication: From subspace methods to compressed sensing," IEEE Trans. Signal Process., vol. 58, no. 3, pp. 17081721, Mar. 2010.

Burdic, W. S., Underwater Acoustic Systems Analysis. Peninsula Publishing, 1990, vol. 2nd Edition.

Caire, G., Al-Naffouri, T., and Narayanan, A., "Impulse noise cancellation in OFDM: An application of compressed sensing," in Proc. IEEE Int. Symp. Inf. Theory, 2008, pp. 1293-1297.

Catipovic, J., "Robust acoustic communication with underwater vehicles in noisy and jammed shallow water environments," in OCEANS 1992: Mastering the Oceans Through Technology, 1992.

Catipovic, J. A., "Performance limitations in underwater acoustic telemetry," IEEE Journal of Oceanic Engineering, vol. 15, no. 3, pp. 205-216, 1990.

Ceballos, P. and Stojanovic, M., "Adaptive channel estimation and data detection for underwater acoustic MIMO OFDM systems," IEEE J. Ocean Eng., vol. 35, no. 3, pp. 635-646, Jul. 2010. 
Chen, W., "Physical layer design considerations for underwater acoustic sensor networks," in in Computer Science and Information Technology (ICCSIT), 3rd International Conference on, 2010.

Chiani, M., Win, M. Z., Zanella, A., Mallik, R. K., and Winters, J. H., "Bounds and approximations for optimum combining of signals in the presence of multiple cochannel interferers and thermal noise," IEEE Transactions on Communications, vol. 51, no. 2, pp. 296-306, Feb. 2003.

Chitre, M., Ong, S. H., and Potter, J., "Performance of coded OFDM in very shallow water channels and snapping shrimp noise," in Proc. MTS/IEEE OCEANS vol 2, 2005.

Chitre, M., Shahabudeen, S., Freitag, L., and Stojanovic, M., "Recent advances in underwater acoustic communications \& networking," in Proc. OCEANS 2008, vol. 2008-Supplement, no. 10.1109/OCEANS.2008.5289428, 2008, pp. 1-10.

Darsena, D., "Successive narrowband interference cancellation for OFDM systems," IEEE Commun. Lett., vol. 11, pp. 73-75, 2007.

Darsena, D. and Verde, F., "Successive NBI cancellation using soft decisions for OFDM systems," IEEE Signal Process. Lett., vol. 15, pp. 873-876, 2008.

Fazel, K., "Narrow-band interference rejection in orthogonal multicarrier spreadspectrum communications," in Third Annual International Conference on Universal Personal Communications, 1994, pp. 46-50.

Freitag, L., Stojanovic, M., Kilfoyle, D., and Presig, J., "A review of a decade of research and a perspective on future challenges," in Proc. of 7th Europan Conference on Underwater Acoustics, 2004.

García, M. F.-G., Rojo-Álvarez, J., Alonso-Atienza, F., and Martínez-Ramón, M., "Support vector machines for robust channel estimation in OFDM," IEEE Signal Process. Lett., vol. 13, no. 7, pp. 397-400, 2006.

Gendron, P. J., "Orthogonal frequency division multiplexing with on-off-keying: Noncoherent performance bounds, receiver design and experimental results," U. S. Navy Journal of Underwater Acoustics, vol. 56, no. 2, pp. 267-300, 2006.

Ghosh, M., "Analysis of the effect of impulse noise on multicarrier and single carrier QAM systems," IEEE Trans. Commun., vol. 44, no. 2, pp. 145-147, 1996.

Goddard, R. P., The Sonar Simulation Toolset, Release 4.1: Science, Mathematics and Algorithms. Seattle, WA: APL-UW TR 0404, Applied Physics Laboratory University of Washington, April 2004.

Goldsmith, A., Wireless Communications. Cambridge Univsersity Press, 2005. 
Gomaa, A., Islam, K. M. Z., and Al-Dhahir, N., "Two novel compressive-sensing algorithms for NBI detection in OFDM systems," in Proc. Int. Conf. on Acoust., 2010, pp. 3294-3297.

Green, D., "Acoustic modems, navigation aids, and networks for undersea operations," in Proc. IEEE Oceans Conference, Marco Island, FL, Jan. 2010.

Hayford, R., Nagle, D., and Catipovic, J., "Undersea cellular network, Tongue of the Ocean Bahamas," in Proc. of the 4th ACM Workshop on Underwater Network (WuWNet), Berkerly, 2009.

He, F., Wang, X., Yang, T., Hu, B., and Liu, R., "Two blind algorithms of MMSE filter based on second-order statistics in MIMO systems with multiple interferences," in Wireless Communications and Signal Processing (WCSP), 2011.

Heidermann, J., Stojanovic, M., and Zorzi, M., "Underwater sensor networks: Applications, advances, and challenges," Royal Society, 2012.

Huang, J., Huang, J.-Z., Berger, C. R., Zhou, S., and Willett, P., "Iterative sparse channel estimation and decoding for underwater MIMO-OFDM," in EURASIP J. on Adv. Signal Process., 2010.

Huang, J., Zhou, S., and Willett, P., "Nonbinary LDPC coding for multicarrier underwater acoustic communication," IEEE J. Sel. Areas Commun., vol. 26, no. 9, pp. 1684-1696, Dec. 2008.

Huang, J. Z., Zhou, S., Huang, J., Berger, C. R., and Willett, P., "Progressive intercarrier interference equalization for OFDM transmission over time-varying underwater acoustic channels," in Proc. MTS/IEEE Oceans Conference, 2010.

Jensen, F. B., Kuperman, W. A., Porter, M. B., and Schmidt, H., Computational Ocean Acoustics. Springer, 2000, vol. 2nd Edition.

Jeremic, A., Thomas, T. A., and Nehorai, A., "OFDM channel estimation in the presence of interference," IEEE Trans. Signal Process., vol. 52, no. 12, pp. 3429-3439, 2004.

Johnson, S. J., Iterative Error Correction: Turbo, Low Density Parity-Check and Repeat Accumulate Codes. New South Wales: Cambridge University Press, 2010.

Kand, T. and Iltis, R. A., "Iterative carrier frequency offset and channel estimation for underwater acoustic OFDM systems," IEEE J. Sel. Areas Commun., vol. 26, no. 9, pp. 1650-1661, Dec. 2008.

Kang, T., Song, H. C., Hodgkiss, W. S., and Kim, J. S., "Long-range multi-carrier acoustic communications in shallow water based on iterative sparse channel estimation," J. Acous. Soc. Amer., vol. 128, no. 6, Dec. 2010. 
Kilfoyle, D. B. and Baggeroer, A. B., "The state of the art in underwater acoustic telemetry," IEEE Journal of Oceanic Engineering, vol. 25, no. 1, pp. 4-27, 2000 .

Kim, B. C. and Lu, I. T., "Parameter study of OFDM underwater communications system," in Proc. MTS/IEEE Oceans, Providence, RI, 2000.

Kuperman, W. A., Porter, M. B., Perkins, J. S., and Evans, R. B., "Rapid computation of acoustic fields in three-dimensional ocean environments," J. Acoust. Soc. Am., vol. 89, pp. 125-1133, 1991.

Laster, J. and Reed, J., "Interference rejection in digital wireless communications," IEEE Signal Process. Mag., vol. 14, no. 3, pp. 37-62, 1997.

Leus, G. and Walree, P. V., "Nonbinary LDPC coding for multicarrier underwater acoustic communication," IEEE J. Sel. Areas Commun., vol. 26, no. 9, pp. 1662-1673, Dec. 2008.

Li, B., Huang, J., Zhou, S., Ball, K., Stojanovic, M., Freitag, L., and Willett, P., "MIMO-OFDM for high rate underwater acoustic OFDM systems," IEEE J. Ocean Eng, vol. 34, no. 4, Oct. 2009.

Li, B., Zhou, S., Huang, J., and Willett, P., "Scalable OFDM design for underwater acoustic communication," in ICASSP 2008, Las Vegas, Nevada, USA, 2008.

Li, B., Zhou, S., Stojanovic, M., Freitag, L., and Willett, P., "Multicarrier communications over underwater acoustic channels with nonuniform doppler shifts," IEEE J. Ocean Eng, vol. 33, no. 2, 2008.

Li, T., Mow, W. H., Lau, V., Siu, M., Cheng, R., and Murch, R., "Robust joint interference detection and decoding for OFDM-based cognitive radio systems with unknown interference," IEEE J. Sel. Areas Commun., vol. 25, no. 3, pp. 566-575, 2007.

Li, T., Mow, W. H., and Siu, M. H., "A joint approach to erasure marking and Viterbi decoding for impulsive noise channels," in Proc. IEEE Workshop on Signal Process, 2003.

Ma, X., Tepedelenlioglu, C., Giannakis, G. B., and Barbarossa, S., "Nondataaided carrier offset estimations for OFDM with null subcarriers: Identifiability, algorithms, and performance," IEEE J. Sel. Areas Commun., vol. 19, no. 12, pp. 2504-2515, 2001.

Mason, S., Berger, C. S., Zhou, S., and Willett, P., "Detection, synchronization, and doppler scale estimation with multicarrier waveforms in underwater acoustic communication," IEEE J. Sel. Areas Commun., vol. 26, no. 9, 2008. 
Mason, S. F., Berger, C. R., Zhou, S., and Willet, P., "Detection, synchronization, and doppler scale estimation with multicarrier waveformsin underwater acoustic communications," IEEE J. Sel. Areas Commun., vol. 26, no. 9, pp. 1638-1649, 2008.

Mengi, A. and Vinck, A., "Successive impulsive noise suppression in OFDM," in Proc. IEEE Int. Symp. on Power Line Commun. Appl., 2010.

Morelli, M. and Moretti, M., "Channel estimation in OFDM systems with unknown interference," IEEE Trans. Wireless Commun., vol. 8, no. 10, pp. 5338-5347, 2009.

Morelli, M. and Moretti, M., "Improved decoding of BICM-OFDM transmissions plagued by narrowband interference," IEEE Trans. Wireless Commun., vol. 10, no. 1, pp. 20-26, 2011.

Muquet, B., Wang, Z., and Giannakis, G., "Cyclic prefixing or zeropadding for wireless multicarrier transmissions?" IEEE Trans. Commun., vol. 50, no. 12, pp. 2136-2148, Dec. 2002.

Negi, R. and Cioffi, J., "Pilot tone selection for channel estimation in a mobile OFDM system," IEEE Trans. Consumer Electronics, vol. 44, no. 3, Aug 1998.

Nilsson, R., Sjoberg, F., and LeBlanc, J., "A rank-reduced LMMSE canceller for narrowband interference suppression in OFDM-based system," IEEE Trans. Commun., vol. 51, no. 12, pp. 2126-2140, 2003.

Partan, J., Kurose, J., and Levine, B. N., "A survey of practical issues in underwater networks," in Proc. ACM WUWNet, 2006.

Pelekanakis, K., "Harvesting time-frequency-space diversity with coded modulation for underwater acoustic communications," Ph.D. dissertation, Massachusetts Institute of Technology, 2009.

Pelekanakis, K., Liu, H., and Chitre, M., "An algorithm for sparse underwater acoustic channel identification under symmetric alpha-stable noise," in Proc. of MTS/IEEE OCEANS Conf., 2011.

Perkins, J. S., Kuperman, W., Ingenito, F., and Glattetre, J., "Modeling ambient noise in three-dimensional ocean environments," J. Acoust. Soc. Am., vol. 93, pp. 739-752, 1993.

Porter, M. B., "A numerical method for computing ocean acoustic modes," Ph.D. dissertation, Northwestern University, 1984.

Porter, M. B. and Reiss, E. L., "A numerical method for ocean acoustic normal modes," J. Acoust. Soc. Am., vol. 76, pp. 244-252, 1984. 
Porter, M. B. and Reiss, E. L., "A numerical method for acoustic normal modes for shear flows," J. Sound and Vibration, vol. 100, pp. 91-105, 1985.

Porter, M. B. and Reiss, E. L., "A numerical method for bottom interacting ocean acoustic normal modes," J. Acoust. Soc. Am., vol. 77, pp. 1760-1767, 1985.

Porter, M. B. and Liu, Y. C., "Finite-element ray tracing," Theoretical and Computational Acoustics, vol. 2, pp. 947-957, September 1994.

Presig, J. C. and Brady, D., "Underwater acoustic communications," in Wireless Communications. New Jersey: Prentice Hall, 1998.

Proakis, J. and Masoud, S., Digital Communications, 5th ed. McGraw-Hill, 2008.

Proakis, J., Soxer, E., Rice, J., and Stojanovic, M., "Shallow water acoustic networks," IEEE Communications Magazine, vol. 39, no. 11, pp. 114-119, 2001.

Qarabaqi, P. and Stojanovic, M., "Statistical characterization and computationally efficient modeling of a class of underwater acoustic communication channels," IEEE Journal of Oceanic Engineering, vol. 38, no. 4, pp. 701-717, Oct. 2013.

Qu, F. and Yang, L., "Basis expansion model for underwater acoustic channels," in Proc. MTS/IEEE OCEANS Conf, Quebec City, 2008.

Qu, F. and Yang, L., "Rate and reliability oriented underwater acoustic communication schemes," in Proc. 2009 DSP \& SPE Workshop, Marco Island, FL, Jan. 2009.

Roy, S., Duman, T. M., McDonald, V., and Proakis, J. G., "High rate communication for underwater acoustic channels using multiple transmitters and spacetime coding: Receiver structures and experimental results," IEEE J. Ocean. Eng., vol. 32, no. 3, pp. 663-688, Jul. 2007.

Song, H.-C., "Long-range acoustic communication in deep water using a towed array: Beam diversity," in OCEANS, Yeosu 2012.

Stojanovic, M., "Recent advances in high-speed underwater acoustic communications," IEEE J. Oceanic Eng., vol. 21, no. 2, pp. 125-126, 1996.

Stojanovic, M., "Underwater acoustic communication," in Encyclopedia of Electrical and Electronics Engineering. Wiley, 2000.

Stojanovic, M., "Low complexity OFDM detector for underwater channels," in Proc. MTS/IEEE OCEANS conference, Boston, 2006.

Stojanovic, M., Catipovic, J. A., and Proakis, J. G., "Adaptive multichannel combining and equalization for underwater acoustic communications," J. Acoust. Soc. Amer., vol. 94, no. 3, pp. 1621-1631, 1993. 
Stojanovic, M. and Presig, J., "Underwater communication channels: Propagation models and statistical characterization," IEEE Communications Magazine, pp. 84-89, January 2009.

Stojanovic, M., "OFDM for underwater acoustic communications: Adaptive synchronization and sparse channel estimation," ICASSIP, p. 5288, 2008.

Sun, H., Shen, W., Wang, Z., Zhou, S., Xu, X., and Chen, Y., "Joint carrier frequency offset and impulse noise estimation for underwater acoustic OFDM with null subcarriers," in Proc. IEEE Oceans Conference, 2012.

Tu, K., Fertonani, D., Duman, T. M., and Hursky, P., "Mitigation of intercarrier interfereence in OFDM systems over underwater acoustic channels," in Proc. MTS/IEEE OCEANS, Bremen, Germany, May 2009.

Tuchler, M., Koetter, R., and Singer, A. C., "Turbo equalization: Principle and new results," IEEE Trans. Commun., vol. 50, no. 5, pp. 754-766, 2002.

Tuchler, M., Singer, A. C., and Koetter, R., "Minimum mean squared error equalization using a priori information," IEEE Trans. Signal Process., vol. 50, no. 3, pp. 673-683, 2002.

Vaccaro, R. J., "The past, present and future of underwater acoustic signal processing," IEEE Signal Processing Magazine, vol. 15, no. 4, pp. 21-51, 1998.

Vasilescu, I., Kotay, R., Rus, D., Dunbabin, M., and Corke, P., "Data collection, storage, and retrieval with an underwater sensor network," in Proc. of the third ACM SenSys conference, no. 3, San Diego, June 2005, pp. 154-165.

Wang, Z., Shengli, Z., Catipovic, J., and Willett, P., "Asynchronous multiuser reception for OFDM in underwater acoustic communications," IEEE Transactions on Wireless Communications., vol. 12, no. 3, pp. 1050-1061, Mar. 2013.

Wang, Z., Zhou, S., Catipovic, J., and Willett, P., "Parameterized cancellation of partial-band partial-block-duration interference for underwater acoustic OFDM," IEEE Transactions on Signal Processing, vol. 60, no. 4, pp. 1782$1795,2012$.

Ward, J., Fitzpatrick, M., Dimarzio, N., Moretti, D., and Morrizzey, R., "New algorithm for open ocean mammal monitoring," in Proc. MTS/IEEE OCEANS Conf., 2000.

Winters, J. H., "Optimum combining in digital mobile radio with cochannel interference," no. 2, 1984, pp. 528-539. 
Xu, X., Zhou, S., Sun, H., Morozov, A. K., and Zhang, Y., "Impulsive noise suppression in per survivor processing based DSSS systems," in Proc. IEEE Oceans Conference, St John's Newfoundland Canada, September 2014.

Yerramalli, S. and Mitra, U., "Optimal resampling of OFDM signals for multiscale - multilag underwater acoustic channels," IEEE J. Ocean. Eng., vol. 36, no. 1, pp. 126-138, 2011.

Yu, X., Lin, P., He, Z., and Wu, W., "OFDM channel estimation with impulse noise cancellation," in Proc. Int. Conf. on Wireless Commun., 2007, pp. 330-333.

Zheng, Y. R., Xiao, C., Yang, T. C., and Yang, W. B., "Frequency-domain channel estimation and equalization for shallow-water acoustic communications," Elsevier J. Phys. Commun., vol. 3, pp. 48-63, Mar. 2010.

Zhidkov, S., "Impulsive noise suppression in OFDM-based communication systems," IEEE Trans. Consum. Electron., vol. 49, no. 4, pp. 944-948, 2003.

Zhidkov, S. V., "Performance analysis and optimization of OFDM receiver with blanking nonlinearity in impulsive noise environment," IEEE Trans. Vehicular Technology, vol. 55, no. 1, pp. 234-242, Jan. 2006Jan.

Zhu, Y., Guo, D., and Honig, M. L., "A message-passing approach for joint channel estimation, interference mitigation, and decoding," IEEE Trans. Wireless Commun., vol. 8, no. 12, pp. 6008-6018, 2009.

Zogakis, T., Chow, P., Aslanis, J., and Cioffi, J., "Impulse noise mitigation strategies for multicarrier modulation," in Proc. Int. Conf. Commun., 1993, pp. $784-788$. 